\title{
Measurements of $\mathbf{t} \overline{\mathbf{t}}$ differential cross sections in proton-proton collisions at $\sqrt{\mathrm{s}}=13 \mathrm{TeV}$ using events containing two leptons
}

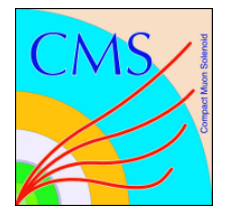

\section{The CMS collaboration}

E-mail: cms-publication-committee-chair@cern.ch

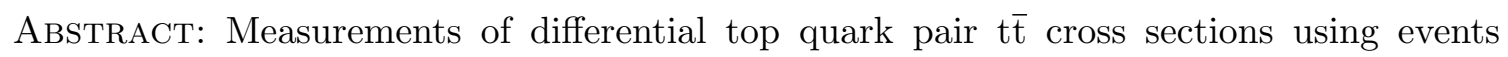
produced in proton-proton collisions at a centre-of-mass energy of $13 \mathrm{TeV}$ containing two oppositely charged leptons are presented. The data were recorded by the CMS experiment at the CERN LHC in 2016 and correspond to an integrated luminosity of $35.9 \mathrm{fb}^{-1}$. The differential cross sections are presented as functions of kinematic observables of the top quarks and their decay products, the t $\bar{t}$ system, and the total number of jets in the event. The differential cross sections are defined both with particle-level objects in a fiducial phase space close to that of the detector acceptance and with parton-level top quarks in the full phase space. All results are compared with standard model predictions from Monte Carlo simulations with next-to-leading-order (NLO) accuracy in quantum chromodynamics (QCD) at matrix-element level interfaced to parton-shower simulations. Where possible, parton-level results are compared to calculations with beyond-NLO precision in QCD. Significant disagreement is observed between data and all predictions for several observables. The measurements are used to constrain the top quark chromomagnetic dipole moment in an effective field theory framework at NLO in QCD and to extract $t \bar{t}$ and leptonic charge asymmetries.

KEYworDs: Hadron-Hadron scattering (experiments), Top physics

ARXIV EPRINT: 1811.06625 


\section{Contents}

1 Introduction 1

2 The CMS detector 3

3 Event simulation $\quad 4$

4 Event selection $\quad 5$

$\begin{array}{lll}5 & \text { Differential cross section extraction } & 7\end{array}$

6 Systematic uncertainties $\quad 10$

$\begin{array}{lll}6.1 & \text { Experimental sources of uncertainty } & 11\end{array}$

$\begin{array}{ll}6.2 \text { Theoretical sources of uncertainty } & 12\end{array}$

7 Results 13

$\begin{array}{lll}7.1 & \text { Measured observables } & 13\end{array}$

$\begin{array}{lll}7.2 & \text { Theoretical predictions } & 15\end{array}$

$\begin{array}{lll}7.3 & \text { Commentary on results } & 16\end{array}$

8 Constraining the top quark CMDM 39

$9 \quad$ Extraction of the top quark charge asymmetries $\quad 53$

10 Summary $\quad 53$

A Tables of parton-level differential cross sections $\quad 56$

B Tables of particle-level differential cross sections $\quad 62$

$\begin{array}{ll}\text { C Tables of } \chi^{2} / \text { dof and } p \text {-values } & 79\end{array}$

$\begin{array}{ll}\text { The CMS collaboration } & 91\end{array}$

\section{Introduction}

The top quark could play a central role in the electroweak symmetry breaking mechanism of the standard model (SM) and in numerous physics models beyond the SM (BSM). Measurements of the differential production cross sections for top quark pair $(t \bar{t})$ production test SM predictions, while also probing scenarios of BSM physics. Such measurements also allow determination of the top quark mass $\left(m_{\mathrm{t}}\right)$, the strong coupling constant $\left(\alpha_{S}\right)$, and the parton distribution functions (PDFs) of the proton. In this paper the dilepton decay 
channel of the têt process is utilised. Although the dilepton channel has a relatively small branching fraction, it has significantly lower backgrounds than the other $t \bar{t}$ decay channels. As a consequence of the excellent lepton energy resolution, the precise measurement of kinematic observables based on lepton pairs is unique to the dilepton channel. However, because of the presence of two neutrinos in the final state, the measurement of top quark kinematic observables in the dilepton channel requires specialised kinematic reconstruction techniques.

Differential t $\bar{t}$ cross sections have been measured by the ATLAS and CMS collaborations at the CERN LHC in proton-proton (pp) collisions at centre-of-mass energies of 7, 8, and $13 \mathrm{TeV}$ [1-19]. In this paper, measurements of absolute and normalised differential $\mathrm{t} \overline{\mathrm{t}}$ cross sections are presented. The measurements are based on either particle-level objects and extrapolated to a fiducial phase space close to that of the detector acceptance or on parton-level top quarks extrapolated to the full phase space. In the absolute measurements, the integrated and differential cross sections are measured simultaneously. In the normalised measurements, the integrated cross sections are not measured and the uncertainties affecting only the normalisations of the differential cross sections in both data and predictions are reduced. The measurements represent the most comprehensive study of $t \overline{\mathrm{t}}$ production in the dilepton channel to date. Furthermore, this work includes a more detailed treatment of the systematic uncertainties compared to previous CMS measurements of the t $\overline{\mathrm{t}}$ differential cross sections in the dilepton channel at centre-of-mass energies of 7 and $8 \mathrm{TeV}$, and approximately 17 times more data than the previous CMS measurement at $13 \mathrm{TeV}$ using data recorded in 2015 .

The analysis utilises a $13 \mathrm{TeV}$ data sample recorded by the CMS experiment in 2016, corresponding to an integrated luminosity of $35.9 \mathrm{fb}^{-1}[20]$. The differential cross sections are presented as a function of kinematic observables of the top quarks, their decay products, the $t \bar{t}$ system, and the number of jets in the event.

Results presented at the parton level may be compared to state-of-the-art SM calculations with beyond next-to-leading-order (NLO) precision in quantum chromodynamics (QCD) [21-24]. These comparisons facilitate the extraction of $m_{\mathrm{t}}, \alpha_{S}$, and the PDFs. Both parton- and particle-level results may be compared to theoretical predictions from Monte Carlo (MC) generators. The particle-level results do not exhibit the theoretical model dependence of the parton-level results introduced when extrapolating to an experimentally inaccessible phase-space region. This reduced model dependence allows calibration of parton-shower, hadronisation, and underlying-event models within MC generators.

Numerous BSM scenarios that lead to modifications of the t $\bar{t}$ differential cross sections involve the production of new states, e.g. top squarks or Z' bosons [25-27]. The absence of signals of such new states in the LHC data recorded so far suggests that BSM physics might only be directly manifested at an energy scale that is large with respect to the typical scales probed at the LHC. In this case, the new states are only produced virtually at the LHC. These virtual effects can modify the rates and kinematic properties of $t \bar{t}$ events. Theoretically, these effects can be accommodated by adding higher-dimensional operators to the SM Lagrangian in an effective field theory (EFT). The reduced model dependence of the particle-level results makes them particularly suitable to constrain BSM theories. 
The generation of predictions for the particle-level observables does not require any detector simulation. Hence, such measurements facilitate a future, global analysis of EFT operators using multiple observables measured by more than one experiment to simultaneously constrain all relevant EFT operators. An anomalous top quark chromomagnetic dipole moment (CMDM) is a feature of BSM models such as two-Higgs-doublet models, supersymmetry, technicolor, and top quark compositeness models [28, 29]. In this paper, the measured particle-level differential t $\bar{t}$ cross section as a function of the azimuthal angle between the two charged leptons is used to constrain the CMDM in an EFT framework. Signals of BSM physics could also appear in t $\overline{\mathrm{t}}$ production as anomalous top quark or leptonic charge asymmetries. Hence, we extract these quantities from differential t $\bar{t}$ cross section measurements as a function of the difference in absolute rapidity between the top quark and antiquark, and the difference in absolute pseudorapidity between the charged leptons.

The paper is organised as follows. In section 2, a brief description of the CMS detector is provided. In section 3, the simulation of signal and background processes is detailed, followed by the description of the selection of events at the trigger level and in the offline analysis in section 4 . The sources of systematic uncertainties that affect the measurements are discussed in section 6 , along with the methods employed to estimate the size of their effects. In section 5, details of the objects and phase-space regions used to define the measured observables are provided, together with a description of the unfolding procedure used to determine the particle- and parton-level data. The unfolded data are presented and compared to theoretical predictions in section 7 . In sections 8 and 9 , constraints on the top quark CMDM in an EFT framework and the t $\bar{t}$ and leptonic charge asymmetries are derived from the unfolded data. Finally, the paper is summarised in section 10.

\section{The CMS detector}

The central feature of the CMS apparatus is a superconducting solenoid of $6 \mathrm{~m}$ internal diameter, providing a magnetic field of $3.8 \mathrm{~T}$. Within the solenoid volume are a silicon pixel and strip tracker, a lead tungstate crystal electromagnetic calorimeter (ECAL), and a brass and scintillator hadron calorimeter, each composed of a barrel and two endcap sections. Forward calorimeters extend the pseudorapidity coverage provided by the barrel and endcap detectors. Muons are measured in gas-ionisation detectors embedded in the steel flux-return yoke outside the solenoid. Events of interest are selected using a twotiered trigger system [30]. The first level, composed of custom hardware processors, uses information from the calorimeters and muon detectors to select events at a rate of around $100 \mathrm{kHz}$ within a time interval of less than $4 \mu \mathrm{s}$. The second level, known as the highlevel trigger (HLT), consists of a farm of processors running a version of the full event reconstruction software optimised for fast processing, and reduces the event rate to around $1 \mathrm{kHz}$ before data storage. A more detailed description of the CMS detector, together with a definition of the coordinate system used and the relevant kinematic variables, can be found in ref. [31]. 


\section{Event simulation}

The simulation of physics processes is important in order to estimate event reconstruction and selection efficiencies, resolutions of the event reconstruction, and to provide predictions for the t $\overline{\mathrm{t}}$ signal and backgrounds. This motivates the use of $\mathrm{MC}$ generators interfaced to a detector simulation. The default simulation setup for the t $\bar{t}$ process is provided at NLO in QCD at the matrix-element (ME) level by the POWHEG (v.2) [32-35] generator (POWHEG). For this setup, the $h_{\text {damp }}$ parameter of POWHEG, which regulates the damping of real emissions in the NLO calculation when matching to the parton shower, is set to $1.58 m_{\mathrm{t}}=272.72 \mathrm{GeV}$ as a result of the tuning of this parameter from ref. [36]. The generated events are subsequently processed with the PYTHIA (v. 8.219) [37] program (PYTHIA), with the CUETP8M2T4 tune [36, 38, 39], for parton showering and hadronisation. In order to compare the predictive powers of alternative ME, parton shower, and hadronisation models, two additional samples are generated using different generator setups. Firstly, a sample is generated using the MADGRAPH5_aMC@NLO [40] (v. 2.2.2) (MG5_aMC@NLO) generator including up to two extra partons at the ME level with NLO precision. In this setup, referred to as "MG5_amC@NLO+PYTHIA[FXFX]", MADSPIN [41] is used to model the decays of the top quarks, while preserving their spin correlation, and events are matched to PYTHIA for parton showering and hadronisation using the FxFx prescription [42]. Secondly, a sample is generated with POWHEG at NLO in QCD at the ME level and interfaced with HERWIG ++ (v. 2.7.1) [43] with the EE5C tune [44] for parton showering and hadronisation. This setup is referred to as POWHEG+HERWIG ++ .

Only t $\bar{t}$ events with two electrons or muons that do not originate from the decays of $\tau$ leptons or semileptonic $\mathrm{b}$ hadron decays are considered as signal, with all other $\mathrm{t} \overline{\mathrm{t}}$ events regarded as a background, which we refer to as "t $\bar{t}$ other". The largest background contributions originate from $\mathrm{t} \overline{\mathrm{t}}$ other, single top quarks produced in association with a $\mathrm{W}$ boson (tW), $\mathrm{Z} / \gamma^{*}$ bosons produced with additional jets ( $\mathrm{Z}+$ jets), $\mathrm{W}$ boson production with additional jets $(\mathrm{W}+$ jets), diboson $(\mathrm{WW}, \mathrm{WZ}$, and $\mathrm{ZZ}$ ) events, and the production of a $\mathrm{t} \overline{\mathrm{t}}$ pair in association with a $\mathrm{Z}$ or $\mathrm{W}$ boson $(\mathrm{t} \overline{\mathrm{t}}+\mathrm{Z} / \mathrm{W})$. Other backgrounds are negligible in comparison to the uncertainties in the main backgrounds. The $\mathrm{W}+$ jets process is simulated at leading-order (LO) precision using MG5_aMC@NLO with up to four additional partons at ME level and matched to PYTHIA using the MLM prescription [45]. The Z+jets process is simulated at NLO precision using MG5_aMC@NLO with up to two additional partons at ME level and matched to PYTHIA using the FxFx prescription. The $t \bar{t}+\mathrm{Z} / \mathrm{W}$ processes are simulated with MG5_aMC@NLO with NLO precision at ME level and matched to PYTHIA. In the case of $t \bar{t}+W$, one extra parton is simulated at ME level and the calculation is matched to PYTHIA using the FxFx prescription. Single top quark production is simulated with POWHeg (v. 1) $[46,47]$ using the CUETP8M2T4 tune in PYTHIA. Diboson events are simulated with PYTHIA.

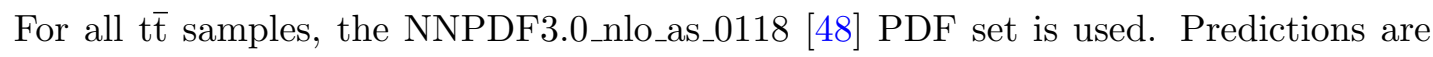
normalised based on their theoretical cross sections and the integrated luminosity of the data. The cross sections are calculated at the highest orders of perturbative QCD currently available. This corresponds to next-to-NLO (NNLO) for $\mathrm{W}+$ jets and $\mathrm{Z}+$ jets [49], 
approximate NNLO for single top quark in the tW channel [50], and NLO calculations

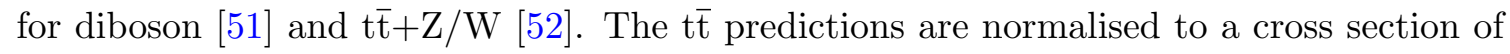
$832_{-29}^{+20}($ scale $) \pm 35\left(\mathrm{PDF}+\alpha_{S}\right)$ pb calculated with the ToP ++2.0 program [53] at NNLO including resummation of next-to-next-to-leading-logarithmic (NNLL) soft-gluon terms, assuming a top quark mass $m_{\mathrm{t}}=172.5 \mathrm{GeV}$. Additional proton-proton interactions within the same or nearby bunch crossings (pileup) is simulated for all samples. The interactions of particles with the CMS detector is simulated using GEANT4 (v. 9.4) [54].

\section{Event selection}

The event selection procedure is designed to select events corresponding to the decay topology where both top quarks decay into a $\mathrm{W}$ boson and a bottom quark (b quark), and each of the $\mathrm{W}$ bosons decays into a muon or an electron, and a neutrino. Three distinct channels based on the flavours of the final-state leptons are defined: the same-flavour channels corresponding to two electrons $\left(\mathrm{e}^{+} \mathrm{e}^{-}\right)$or two muons $\left(\mu^{+} \mu^{-}\right)$, and the different-flavour channel corresponding to one electron and one muon $\left(\mathrm{e}^{ \pm} \mu^{\mp}\right)$. The final results are derived by combining the three channels. At HLT level, events are selected either by single-lepton triggers that require the presence of at least one electron or muon or by dilepton triggers that require the presence of either two electrons, two muons, or an electron and a muon. For the single-electron and single-muon triggers, transverse momentum $p_{\mathrm{T}}$ thresholds of 27 and $24 \mathrm{GeV}$ are applied, respectively. The same-flavour dilepton triggers require either an electron pair with $p_{\mathrm{T}}>23(12) \mathrm{GeV}$ for the leading (trailing) electron or a muon pair with $p_{\mathrm{T}}>17(8) \mathrm{GeV}$ for the leading (trailing) muon, where leading (trailing) refers to the electron or muon with the highest (second-highest) $p_{\mathrm{T}}$ in the event. The differentflavour dilepton triggers require either a muon with $p_{\mathrm{T}}>23 \mathrm{GeV}$ and an electron with $p_{\mathrm{T}}>12 \mathrm{GeV}$, or an electron with $p_{\mathrm{T}}>23 \mathrm{GeV}$ and a muon with $p_{\mathrm{T}}>8 \mathrm{GeV}$.

The events selected by the trigger are reconstructed offline using a particle-flow algorithm [55]. The particle-flow algorithm aims to reconstruct and identify each individual particle in an event, with an optimised combination of information from the various elements of the CMS detector. Electron candidates are reconstructed from a combination of the track momentum at the main interaction vertex and the corresponding clusters in the ECAL with a Gaussian sum filter algorithm [56]. The electron candidates are required to have $p_{\mathrm{T}}>25(20) \mathrm{GeV}$ for the leading (trailing) candidate and $|\eta|<2.4$. Electron candidates with ECAL clusters in the region between the barrel and endcap $\left(1.44<\left|\eta_{\text {cluster }}\right|<1.57\right)$ are excluded because of less efficient electron reconstruction. A relative isolation criterion $I_{\text {rel }}<0.0588(0.0571)$ is applied for an electron candidate in the barrel (endcap), where $I_{\text {rel }}$ is defined as the sum of the $p_{\mathrm{T}}$ of all neutral hadron, charged hadron, and photon candidates within a distance of 0.3 from the electron in $\eta-\phi$ space, divided by the $p_{\mathrm{T}}$ of the electron candidate. In addition, electron identification requirements are applied to reject misidentified electron candidates and candidates originating from photon conversions. Muon candidates are reconstructed using the track information from the tracker and the muon system [57]. They are required to have $p_{\mathrm{T}}>25(20) \mathrm{GeV}$ for the leading (trailing) candidates and $|\eta|<2.4$. An isolation requirement of $I_{\text {rel }}<0.15$ is applied to muon can- 
didates with particles within 0.4 of the muon in $\eta-\phi$ space included in the calculation of $I_{\text {rel }}$. In addition, muon identification requirements are applied to reject misidentified muon candidates and candidates originating from decay-in-flight processes. For both electron and muon candidates, a correction is applied to $I_{\text {rel }}$ to suppress the residual effect of pileup.

Jets are reconstructed by clustering the particle-flow candidates using the anti- $k_{\mathrm{T}}$ clustering algorithm with a distance parameter of 0.4 [58,59]. Jet momentum is determined as the vectorial sum of all particle momenta in the jet, and is found from simulation to be within 5 to $10 \%$ of the true momentum over the whole $p_{\mathrm{T}}$ spectrum and detector acceptance. Pileup can contribute additional tracks and calorimetric energy deposits to the jet momentum. To mitigate this effect, tracks identified to be originating from pileup vertices are discarded, and an offset correction is applied to correct for remaining contributions. Jet energy corrections are derived from simulation to bring the measured response of jets to that of particle-level jets on average. In situ measurements of the momentum imbalance in dijet, photon+jets, $\mathrm{Z}+$ jets, and multijet events are used to account for any residual differences in jet energy in data and simulation. Additional selection criteria are applied to remove badly reconstructed jets. Jets are selected if they have $p_{\mathrm{T}}>30 \mathrm{GeV}$ and $|\eta|<2.4$. Jets are rejected if the distance in $\eta-\phi$ space between the jet and the closest lepton, $\Delta R$ (jet, lepton), is less than 0.4. Jets originating from the hadronisation of b quarks (b jets) are identified (b tagged) by combining information related to secondary decay vertices reconstructed within the jets and track-based lifetime information in an algorithm CSV (v.2) [60] that provides a b jet identification efficiency of $\approx 79-87 \%$ and a probability to misidentify light-flavour jets as b jets of $\approx 10 \%$.

The missing transverse momentum vector $\vec{p}_{\mathrm{T}}^{\text {miss }}$ is defined as the projection on the plane perpendicular to the beams of the negative vector sum of the momenta of all reconstructed particles in an event. Its magnitude is referred to as $p_{\mathrm{T}}^{\text {miss }}$.

Events are selected offline if they contain exactly two isolated, oppositely charged electrons or muons $\left(\mathrm{e}^{+} \mathrm{e}^{-}, \mu^{+} \mu^{-}, \mathrm{e}^{ \pm} \mu^{\mp}\right)$ and at least two jets. At least one of the jets is required to be $\mathrm{b}$ tagged. Events with an invariant mass of the lepton pair $\left(m_{\ell \bar{\ell}}\right)$ smaller than $20 \mathrm{GeV}$ are removed in order to suppress contributions from heavy-flavour resonance decays and low-mass Drell-Yan processes. Backgrounds from Z+jets processes in the $\mathrm{e}^{+} \mathrm{e}^{-}$ and $\mu^{+} \mu^{-}$channels are further suppressed by requiring $m_{\ell \bar{\ell}}<76 \mathrm{GeV}$ or $m_{\ell \bar{\ell}}>106 \mathrm{GeV}$, and $p_{\mathrm{T}}^{\text {miss }}>40 \mathrm{GeV}$. The normalisation of the remaining background contribution from $\mathrm{Z}+$ jets events, which is large in the $\mathrm{e}^{+} \mathrm{e}^{-}$and $\mu^{+} \mu^{-}$channels, is determined by applying a factor derived from simulation to the number of $\mathrm{Z}+$ jets events observed in data in a control region where $m_{\ell \bar{\ell}}$ is close to $m_{\mathrm{Z}}[8,61]$. A correction to account for non-Z+jets backgrounds in the control region is derived from the $\mathrm{e}^{ \pm} \mu^{\mp}$ channel. The shape of the $\mathrm{Z}+$ jets background is taken from simulation. Other sources of background such as tW, diboson, $t \bar{t}+\mathrm{Z} / \mathrm{W}, \mathrm{t} \overline{\mathrm{t}}$ other, misidentified leptons, and leptons within jets are estimated from simulation.

The kinematic observables of the top quarks are estimated via a kinematic reconstruction algorithm [8]. The algorithm examines all combinations of jets and leptons and solves a system of equations based on the following constraints: $p_{\mathrm{T}}^{\text {miss }}$ is assumed to originate solely from the two neutrinos; the invariant mass of the reconstructed $\mathrm{W}$ boson must 
equal $80.4 \mathrm{GeV}$ [62]; and the invariant mass of each reconstructed top quark must equal $172.5 \mathrm{GeV}$. Effects of detector resolution are accounted for by randomly varying the measured energies and directions of the reconstructed lepton and b jet candidates by their resolutions as measured in simulation. This procedure is referred to in the following to as smearing. In addition, the assumed invariant mass of the $\mathrm{W}$ boson is smeared according to the Breit-Wigner distribution of $\mathrm{W}$ boson masses in simulation. For a given smearing, the solution of the equations for the neutrino momenta yielding the smallest invariant mass of the $t \bar{t}$ system is chosen. For each solution, a weight is calculated based on the spectrum of the true invariant mass of the lepton and $b$ jet system from simulated top quark decays at particle level. The weights are summed over 100 smearings for each combination, and the kinematic observables of the top quark and antiquark are calculated as a weighted average. The smearing procedure increases the fraction of combinations in which a valid solution to the system of equations is found. Increasing the number of smearings beyond 100 did not significantly increase this fraction further. The top quark and antiquark candidates are distinguished according to the charge of the lepton in the chosen solution. The solution with the most b-tagged jets is chosen to represent the top quark momenta. If multiple combinations with the same number of b-tagged jets are found, the combination that yields the maximum sum of weights is chosen. The efficiency of the kinematic reconstruction, defined as the number of events where a solution is found divided by the total number of selected $t \bar{t}$ events, is about $90 \%$ in both data and simulation. Events with no valid solution for the neutrino momenta are excluded from further analysis.

After applying the full event selection, 34890 events in the $\mathrm{e}^{+} \mathrm{e}^{-}$channel, 70346 events in the $\mu^{+} \mu^{-}$channel, and 150410 events in the $\mathrm{e}^{ \pm} \mu^{\mp}$ channel are observed. In all decay channels combined, the estimated signal contribution to the data is $80.6 \%$. In figure 1 , selected distributions of the kinematic observables and multiplicities of the selected jets $\left(N_{\text {jets }}\right)$ and b jets $\left(N_{\mathrm{b} \text { jets }}\right)$ are shown. For each distribution, all event selection criteria are applied, with the exception of the $N_{\mathrm{b}}$ jets distribution where no b-tagged jets are required. Figure 2 shows the distributions of the top quark or antiquark and t $\overline{\mathrm{t}}$ kinematic observables (the transverse momenta $p_{\mathrm{T}}^{\mathrm{t}}, p_{\mathrm{T}}^{\mathrm{t} \overline{\mathrm{t}}}$, the rapidities $y_{\mathrm{t}}, y_{\mathrm{t} \overline{\mathrm{t}}}$, and the invariant mass of the $\mathrm{t} \overline{\mathrm{t}}$ system $\left.m_{\mathrm{t} \overline{\mathrm{t}}}\right)$. The mismodelling of the data by the simulation, apparent in the tails of the distributions, is accounted for by the corresponding systematic uncertainties, as described in section 6. Simulation is used to verify that mismodelling of the $p_{\mathrm{T}}^{\mathrm{t}}$ distribution does not bias the results for the differential cross section as a function of $p_{\mathrm{T}}^{\mathrm{t}}$.

\section{Differential cross section extraction}

For a given variable $X$, the absolute differential t $\overline{\mathrm{t}}$ cross section $\mathrm{d} \sigma_{i} / \mathrm{d} X$ is determined via the relation [8]:

$$
\frac{\mathrm{d} \sigma_{i}}{\mathrm{~d} X}=\frac{1}{\mathcal{L}} \frac{x_{i}}{\Delta_{i}^{X}},
$$

where $\mathcal{L}$ is the integrated luminosity of the data, $x_{i}$ is the number of signal events observed in data for bin $i$ after the background subtraction and correction for the detector efficiencies, acceptances, and bin migration, and $\Delta_{i}^{X}$ is the bin width. The normalised differential 

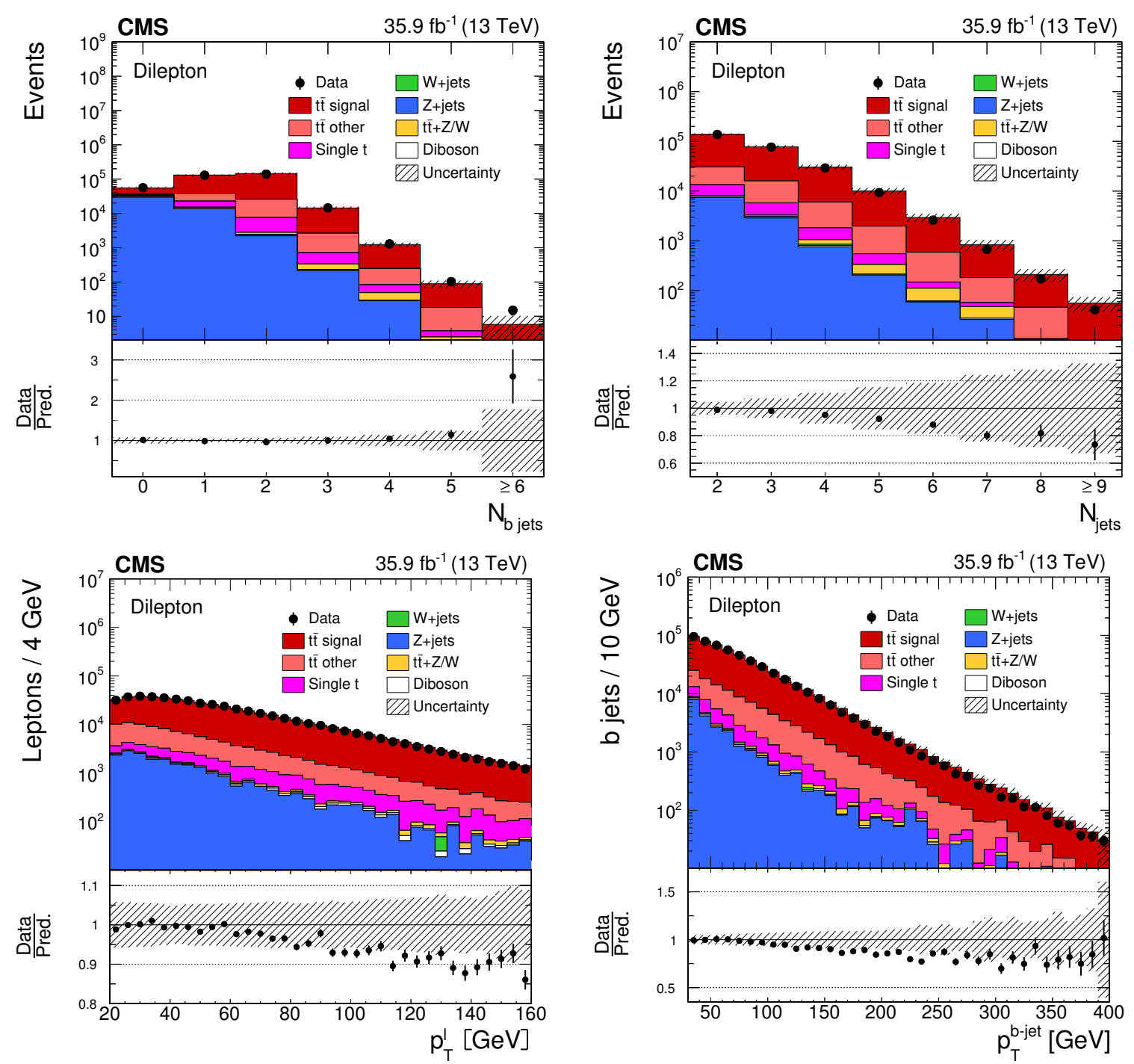

Figure 1. Distributions of the b jet (upper left), and total jet (upper right) multiplicities, and the $p_{\mathrm{T}}$ of the leptons (lower left), and b jets (lower right) are shown for data (points) and simulation

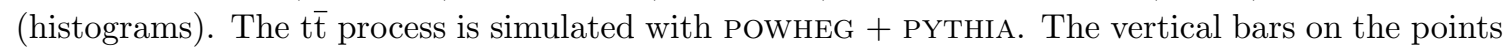
represent the statistical uncertainties in the data. The hatched regions correspond to the systematic uncertainties in the signal and backgrounds, as described in section 6 . The lower panel of each plot shows the ratio of the data to the predictions from simulation.

cross section is obtained by dividing the absolute differential cross section by the measured total cross section $\sigma$ in the same phase space, which is evaluated by summing the binned cross section measurements over all bins of the observable $X$. The background from other $\mathrm{t} \overline{\mathrm{t}}$ decays is taken into account, after subtracting all other background components, by correcting the number of signal events in data using the expected signal fraction. The expected signal fraction is defined as the ratio of the number of selected t $\bar{t}$ signal events to the total number of selected t $\bar{t}$ events in simulation. This procedure avoids the dependence on the total inclusive t $\bar{t}$ cross section used in the normalisation of the simulated signal sample. 

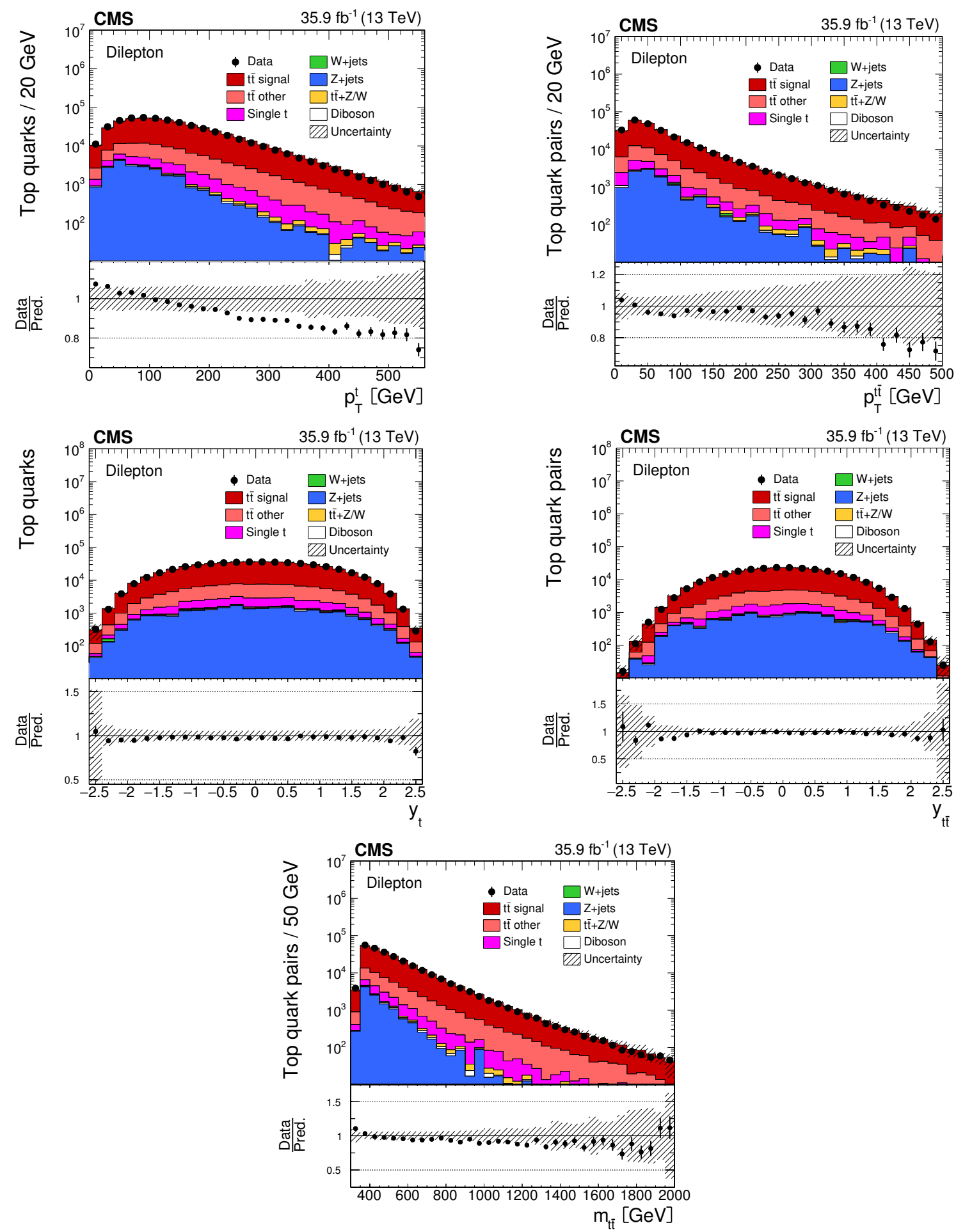

Figure 2. Distributions of the $p_{\mathrm{T}}$ (upper row) and rapidities (middle row), at detector level for the top quarks (left column), and t $\overline{\mathrm{t}}$ system (right column), and $m_{\mathrm{t} \overline{\mathrm{t}}}$ (lower plot) are shown for data (points) and simulation (histograms). The t $\bar{t}$ process is simulated with POWHEG + PYTHIA. The vertical bars on the points represent the statistical uncertainties in the data. The hatched regions correspond to the systematic uncertainties in the signal and backgrounds, as described in section 6 . The lower panel of each plot shows the ratio of the data to the predictions from simulation. 
The finite resolution introduced by the detector response, parton shower, and hadronisation lead to migration of events across bins when correcting the data to both the fiducial phase space based on particle-level objects or the full phase space based on the parton-level top quarks. These effects are accounted for with a regularised unfolding method [8, 63, 64]. For each measured distribution, a response matrix that accounts for migrations and efficiencies is calculated using the default t $\overline{\mathrm{t}}$ simulation. For the parton-level measurements in the full phase space, the response matrix also accounts for the branching fraction of $t \bar{t}$ events into two leptons excluding $\tau$ leptons. The generalised inverse of the response matrix is used to obtain the unfolded distribution from the measured distribution by applying a $\chi^{2}$ minimisation technique. Regularisation is applied to suppress nonphysical fluctuations. The regularisation level is determined individually for each distribution using the average squared global correlation method [65]. To keep the bin-to-bin migrations small, the width of the measurement bins are chosen according to their purity and stability. Purity is defined as the fraction of events in a given bin at the detector level that originate from the same bin at the generator level, and stability is defined as the fraction of events in a given bin at the generator level that are reconstructed in the same bin at the detector level. The purities and stabilities are typically $\approx 50 \%$, except in the regions where the distributions are steeply rising or falling, where values of $30 \%$ are typical. The statistical uncertainty is small in comparison to the systematic uncertainties in all bins. The data in the three channels are combined before unfolding in order to model correlations between channels and reduce statistical uncertainties in poorly populated regions of the unfolding matrix.

For some observables, both the absolute and normalised differential cross sections are measured at both the particle level in a fiducial phase space and at the parton level in the full phase space. This leads to four measurements for each of these observables. The observables related to the kinematics and multiplicities of jets and leptons are determined at the particle level only.

Object and phase-space definitions. The definition of the particle-level objects and the kinematic reconstruction procedure employed to estimate the kinematic properties of the particle-level top quarks are described in ref. [66]. We detail here the additional eventlevel requirements that define the fiducial phase-space region in which the particle-level differential cross sections are measured. We require that the $\mathrm{W}$ bosons produced from decays of the top quark and antiquark in a t $\bar{t}$ event themselves decay to an electron or muon. Events where these $\mathrm{W}$ bosons decay to tau leptons are rejected. The requirements of exactly two selected lepton candidates with opposite charges, a dilepton invariant mass greater than $20 \mathrm{GeV}$, and at least two $\mathrm{b}$ jets are also added.

For the parton-level results, the momenta of the parton-level top quarks are defined after QCD radiation but before the top quark decays. The parton-level results are extrapolated to the full phase space using the default simulation.

\section{Systematic uncertainties}

The systematic uncertainties in the measured differential cross sections are categorised into experimental uncertainties arising from imperfect modelling of the detector response 
and conditions and theoretical uncertainties arising from the modelling of the signal and background processes. Each systematic uncertainty is determined separately in each bin of the measured differential cross section via a variation of the corresponding aspect of the simulation setup.

A regularised unfolding method, described in section 5, is used to correct for the migration of events between bins due to the finite detector resolutions and to extrapolate the detector-level data to the fiducial and full phases spaces. The variations are applied both at detector level and in the response matrices that define the unfolding. For each variation, the difference between the varied and nominal results is taken as the systematic uncertainty. The total systematic uncertainty is calculated by adding these differences in quadrature. In this section, each of these applied variations is detailed.

\subsection{Experimental sources of uncertainty}

In order to account for the differences in trigger efficiencies between data and simulation, scale factors, defined as the ratio of the efficiencies measured in data and simulation, are calculated in bins of lepton $\eta$ and $p_{\mathrm{T}}$ and applied to the simulation. The efficiencies of the dilepton triggers in data are measured as the fraction of events passing triggers based on a $p_{\mathrm{T}}^{\text {miss }}$ requirement that also satisfy the dilepton trigger criteria. As the efficiency of the $p_{\mathrm{T}}^{\text {miss }}$ requirement is independent from the dilepton trigger efficiencies, the bias introduced by the $p_{\mathrm{T}}^{\text {miss }}$ requirement is negligible. The efficiencies are close to unity in both data and simulation. An uncertainty arising from the modelling of the trigger efficiencies in simulation is estimated by two variations of the scale factors. First, the scale factors are varied within their uncertainties coherently for all leptons. Second, to account for potential differential effects not covered by the coherent variations, simulated events are divided into categories according to the $\eta$ of the leptons, and the scale factors are varied in opposite directions for each category. A final trigger uncertainty is derived by taking the maximal deviation produced by the two variations in each bin.

The uncertainties from modelling of the lepton identification and isolation efficiencies are determined using the tag-and-probe method with $\mathrm{Z}+$ jets event samples [61, 67]. The differences between lepton identification and isolation efficiencies in data and simulation in bins of $\eta$ and $p_{\mathrm{T}}$ are generally less than $10 \%$ for electrons, while differences for muons are negligible. The lepton identification uncertainty is estimated by varying the scale factors within their uncertainties.

The uncertainty arising from the jet energy scale (JES) is determined by varying the 19 sources of uncertainty in the JES in bins of $p_{\mathrm{T}}$ and $\eta$ and taking the quadrature sum of the effects [68]. The JES variations are also propagated to the uncertainties in $p_{\mathrm{T}}^{\text {miss }}$. The uncertainty from the jet energy resolution (JER) is determined by the variation of the JER in simulation by \pm 1 standard deviation in different $\eta$ regions [68]. An additional uncertainty from the calculation of $p_{\mathrm{T}}^{\text {miss }}$ is estimated by varying the energies of the reconstructed particles not clustered into jets within their respective resolutions and recalculating the $p_{\mathrm{T}}^{\mathrm{miss}}$.

The uncertainty from the modelling of the number of pileup events is obtained by changing the inelastic proton-proton cross section assumed in simulation by $\pm 4.6 \%$, corresponding to the uncertainty in the measurement of this cross section presented in ref. [69]. 
The uncertainty due to imperfect modelling of the $\mathrm{b}$ tagging efficiency is determined by varying the measured scale factor for $b$ tagging efficiencies within its uncertainties. An additional shape uncertainty is determined by dividing the $\mathrm{b}$ jet distributions in $p_{\mathrm{T}}$ and $\eta$ at their medians to form two bins in each variable. The $b$ tagging scale factors in the first bin are scaled up according to their uncertainties, while those in the second bin are scaled down and vice versa. The variations are performed separately for the $p_{\mathrm{T}}$ and $\eta$ distributions, and independently for heavy-flavour ( $b$ and $c$ ) and light-flavour ( $\mathrm{u}, \mathrm{d}, \mathrm{s}$, and gluon) jets.

The efficiency of the kinematic reconstruction of the top quarks is found to be consistent between data and simulation within around $0.2 \%$. An associated uncertainty is derived by varying the scale factor that describes the ratio of the kinematic reconstruction efficiency in data and simulation by $\pm 0.2 \%$.

The uncertainty in the integrated luminosity of the 2016 data sample recorded by CMS is $2.5 \%$ [20] and is applied coherently to the normalisation of all simulated distributions.

\subsection{Theoretical sources of uncertainty}

The uncertainty arising from the missing higher-order terms in the simulation of the signal process at the ME level is assessed by varying the renormalisation and factorisation scales in the POWHEG simulation up and down by factors of two with respect to their nominal values. In the POWHEG simulation, the nominal scales are defined as $m_{\mathrm{t}}^{2}+p_{\mathrm{T}, \mathrm{t}}^{2}$, where $p_{\mathrm{T}, \mathrm{t}}$ denotes the $p_{\mathrm{T}}$ of the top quark in the $\mathrm{t} \overline{\mathrm{t}}$ rest frame. In total, three variations are applied: one with the factorisation scale fixed, one with the renormalisation scale fixed, and one with both scales varied coherently together. The final uncertainty is taken as the maximum deviation from the nominal prediction from each of the three variations. In the parton-shower simulation, the corresponding uncertainty is estimated by varying the scale of initial- and final-state radiation separately up and down by factors of 2 and $\sqrt{2}$, respectively, as suggested in ref. [39].

The effect of the uncertainty from the choice of PDF is assessed by reweighting the signal simulation according to the prescription provided for the NNPDF3.0 PDF set [48]. An additional uncertainty is independently derived by varying the $\alpha_{S}$ value within its uncertainty in the PDF set. The dependence of the measurement on the assumed $m_{\mathrm{t}}$ value is estimated by varying the chosen $m_{\mathrm{t}}$ in the default setup by $\pm 1 \mathrm{GeV}$ with respect to the default value of $172.5 \mathrm{GeV}$.

The uncertainty originating from the scheme used to match the ME-level calculation to the parton-shower simulation is derived by varying the $h_{\text {damp }}$ parameter in POWHEG by factors of 1.42 and 0.63 , according to the results of a tuning of this parameter from ref. [36].

The uncertainty related to the modelling of the underlying event is estimated by varying the parameters used to derive the CUETP8M2T4 tune in the default setup. The default setup in PYTHIA includes a model of colour reconnection based on multiple-particle interactions (MPI) with early resonance decays switched off. To estimate an uncertainty from this choice of model, the analysis is repeated with three other models of colour reconnection within PYTHIA: the MPI-based scheme with early resonance decays switched on, a gluon-move scheme [70], and a QCD-inspired scheme [71]. The total uncertainty 
from colour reconnection modelling is estimated by taking the maximum deviation from the nominal result.

The uncertainty from imperfect knowledge of the $\mathrm{b}$ quark fragmentation function is assessed by varying the Bowler-Lund function within its uncertainties [72]. In addition, the analysis is repeated with the Peterson model for b quark fragmentation [73]. An uncertainty from the semileptonic branching fraction of $b$ hadrons is estimated by correcting the

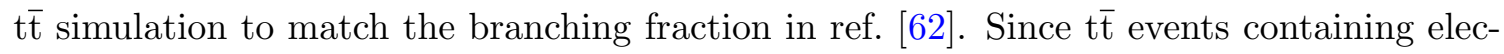
trons or muons that originate from $\tau$ decays are considered as backgrounds, the measured differential cross sections are sensitive to the value of the $\tau$ semileptonic branching fraction used in the simulation. Hence, an uncertainty is derived by varying the branching fractions by $1.5 \%$ [62]. Since the b tagging efficiency depends on many simulation parameters, it is recalculated for each variation of the sources of theoretical uncertainty, with the exception of the PDFs, the semileptonic branching fraction of b hadrons, the JES, and the JER. Finally, the normalisations of all backgrounds except t $\overline{\mathrm{t}}$ other are varied up and down by $\pm 30 \%[61]$.

The total uncertainty in each bin of each measurement is determined by summing the experimental and theoretical uncertainties in quadrature and ranges from $4-25 \%$, depending on the observable and the bin. In section 7, figures showing the contribution of each systematic uncertainty, the statistical uncertainty, and the total uncertainty in each bin for selected normalised parton-level differential cross sections as a function of top-quark-related kinematic observables are provided. For most bins in a majority of these distributions, the JES is the dominant systematic uncertainty. In the first three bins of the $p_{\mathrm{T}}^{\mathrm{t} \mathrm{t}}$ distribution, the dominant uncertainty arises from the measurement of the energies of reconstructed particles not clustered into jets.

\section{Results}

In this section, the results of all differential cross section measurements are presented.

\subsection{Measured observables}

The differential cross sections are measured as functions of numerous kinematic observables of the top quarks and their decay products, the $t \overline{\mathrm{t}}$ system, and the total number of jets in the event. These observables are listed below. For one group of observables both partonand particle-level measurements are presented, while for a second group only particle-level measurements are given.

Observables measured at parton and particle levels:

- $p_{\mathrm{T}}$ of the top quark $\left(p_{\mathrm{T}}^{\mathrm{t}}\right)$

- $p_{\mathrm{T}}$ of the top antiquark $\left(p_{\mathrm{T}}^{\overline{\mathrm{t}}}\right)$

- $p_{\mathrm{T}}$ of the top quark or top antiquark with largest $p_{\mathrm{T}}$ ( $p_{\mathrm{T}}^{\mathrm{t}}$ (leading))

- $p_{\mathrm{T}}$ of the top quark or top antiquark with second-largest $p_{\mathrm{T}}\left(p_{\mathrm{T}}^{\mathrm{t}}\right.$ (trailing))

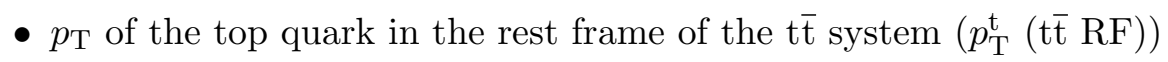


- rapidity of the top quark $\left(y_{\mathrm{t}}\right)$

- rapidity of the top antiquark $\left(y_{\overline{\mathrm{t}}}\right)$

- rapidity of the top quark or top antiquark with largest $p_{\mathrm{T}}$ ( $y_{\mathrm{t}}$ (leading))

- rapidity of the top quark or top antiquark with second-largest $p_{\mathrm{T}}\left(y_{\mathrm{t}}\right.$ (trailing))

- difference in absolute rapidity between the top quark and antiquark $(\Delta|y|(\mathrm{t}, \overline{\mathrm{t}}))$

- absolute difference in azimuthal angle between the top quark and antiquark $(\Delta \phi(\mathrm{t}, \overline{\mathrm{t}}))$

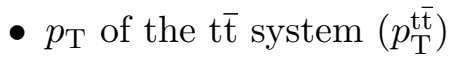

- rapidity of the $\mathrm{t} \overline{\mathrm{t}}$ system $\left(y_{\mathrm{t} \overline{\mathrm{t}}}\right)$

- invariant mass of the $\mathrm{t} \overline{\mathrm{t}}$ system $\left(m_{\mathrm{t} \overline{\mathrm{t}}}\right)$

Observables measured at particle level only:

- $p_{\mathrm{T}}$ of the lepton $\left(p_{\mathrm{T}}^{\ell}\right)$

- $p_{\mathrm{T}}$ of the antilepton $\left(p_{\mathrm{T}}^{\bar{\ell}}\right)$

- $p_{\mathrm{T}}$ of the lepton or antilepton with largest $p_{\mathrm{T}}\left(p_{\mathrm{T}}^{\ell}\right.$ (leading))

- $p_{\mathrm{T}}$ of the lepton or antilepton with second-largest $p_{\mathrm{T}}\left(p_{\mathrm{T}}^{\ell}\right.$ (trailing))

- pseudorapidity of the lepton $\left(\eta_{\ell}\right)$

- pseudorapidity of the antilepton $\left(\eta_{\bar{\ell}}\right)$

- pseudorapidity of the lepton or antilepton with largest $p_{\mathrm{T}}\left(\eta_{\ell}\right.$ (leading))

- pseudorapidity of the lepton or antilepton with second-largest $p_{\mathrm{T}}\left(\eta_{\ell}\right.$ (trailing))

- $p_{\mathrm{T}}$ of the dilepton system $\left(p_{\mathrm{T}}^{\ell \bar{\ell}}\right)$

- invariant mass of the dilepton system $\left(m_{\ell \bar{\ell}}\right)$

- absolute difference in azimuthal angle between the lepton and antilepton $(\Delta \phi(\ell, \bar{\ell}))$

- difference in absolute pseudorapidity between the lepton and antilepton $(\Delta \eta(\ell, \bar{\ell}))$

- $p_{\mathrm{T}}$ of the $\mathrm{b}$ jet with largest $p_{\mathrm{T}}\left(p_{\mathrm{T}}^{\mathrm{b}}\right.$ (leading))

- $p_{\mathrm{T}}$ of the b jet with second-largest $p_{\mathrm{T}}\left(p_{\mathrm{T}}^{\mathrm{b}}\right.$ (trailing))

- pseudorapidity of the $\mathrm{b}$ jet with largest $p_{\mathrm{T}}\left(\eta_{\mathrm{b}}\right.$ (leading))

- pseudorapidity of the $\mathrm{b}$ jet with second-largest $p_{\mathrm{T}}\left(\eta_{\mathrm{b}}\right.$ (trailing))

- $p_{\mathrm{T}}$ of the $\mathrm{b} \overline{\mathrm{b}}$ system $\left(p_{\mathrm{T}}^{\mathrm{b} \overline{\mathrm{b}}}\right)$

- invariant mass of the $\mathrm{b} \overline{\mathrm{b}}$ system $\left(m_{\mathrm{b}} \overline{\mathrm{b}}\right)$

- multiplicity of jets with $p_{\mathrm{T}}^{\text {jet }}>30 \mathrm{GeV}\left(N_{\text {jets }}\right)$

The measurements of top quark $p_{\mathrm{T}}$ are sensitive to higher-order QCD and electroweak corrections in the SM, $m_{\mathrm{t}}$, PDFs, and potential BSM physics signals. In order to probe the modelling of the top quark $p_{\mathrm{T}}$ as thoroughly as possible, various differential cross sections related to the $p_{\mathrm{T}}$ of top quarks are measured. These include: the separate $p_{\mathrm{T}}$ of the top quarks and antiquarks in the laboratory frame and, in order to suppress the effects of 


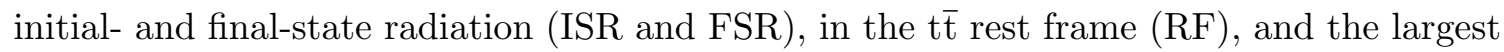
(leading) and second-largest $p_{\mathrm{T}}$ (trailing) top quark or antiquark in an event. Similarly, the rapidity distributions are determined separately for top quarks and antiquarks, as well as the rapidity of the leading and trailing top quark or antiquark in an event. The differential cross sections as a function of the differences in absolute rapidities between the top quark and antiquark and in absolute pseudorapidities between the lepton and antilepton are measured to allow the extraction of the $t \bar{t}$ and leptonic charge asymmetries described in section 9 . The $p_{\mathrm{T}}$ of the t $\overline{\mathrm{t}}$ system and $N_{\text {jets }}$ distributions are measured since they are especially sensitive to the higher-order terms in the perturbative calculations. The rapidity and invariant mass distributions of the t $\bar{t}$ system are measured because of their potential to reduce gluon PDF uncertainties at large fractions of the proton longitudinal momentum carried by the gluon. In addition, for small values of $m_{\mathrm{t} \overline{\mathrm{t}}}$, the $m_{\mathrm{t} \overline{\mathrm{t}}}$ distribution is sensitive to $m_{\mathrm{t}}$, while for large values of $m_{\mathrm{t} \overline{\mathrm{t}}}$, it is sensitive to BSM scenarios in which heavy states decay to $t \bar{t}$ pairs. The measurements of the lepton kinematic observables test the modelling of the top quark decays and spin correlations in the t $\bar{t}$ pair. Measuring the b jet kinematic observables further tests the modelling of the top quark decays, while also testing the parton shower and hadronisation models.

\subsection{Theoretical predictions}

All data are compared to predictions from POWHEG+PYTHIA, POWHEG+HERWIG++, and MG5_aMC@NLO+PYTHIA[FXFX]. Where possible, parton-level measurements are also compared to predictions based on the following calculations at beyond-NLO precision:

- A calculation with full NNLO precision in QCD and including electroweak corrections of order $\alpha_{S}^{2} \alpha_{\mathrm{EW}}, \alpha_{S} \alpha_{\mathrm{EW}}^{2}$, and $\alpha_{\mathrm{EW}}^{3}\left(\mathrm{NNLO}+\alpha_{\mathrm{EW}}^{3}\right)$ [74]. The dynamic renormalisation and factorisation scales are set to $m_{\mathrm{T}} / 2$ for $p_{\mathrm{T}}^{\mathrm{t}}$ and $p_{\mathrm{T}}^{\overline{\mathrm{t}}}$ and $H_{\mathrm{T}} / 4$ for $y_{\mathrm{t}}, y_{\overline{\mathrm{t}}}, p_{\mathrm{T}}^{\mathrm{t} \overline{\mathrm{t}}}, y_{\mathrm{t} \overline{\mathrm{t}}}$, $m_{\mathrm{t} \overline{\mathrm{t}}}$, and $\Delta|y|(\mathrm{t}, \overline{\mathrm{t}})$, where $m_{\mathrm{T}}=\sqrt{m_{\mathrm{t}}^{2}+\left(p_{\mathrm{T}}^{\mathrm{t}}\right)^{2}}$ and $H_{\mathrm{T}}$ is the sum of the top quark and antiquark $m_{\mathrm{T}}$ values. Predictions are provided for both the LUXQED17 [75] and NNPDF3.1_qed PDF [76] sets with $m_{\mathrm{t}}=173.3 \mathrm{GeV}$. In order to probe the sensitivity of the results to the value of $m_{\mathrm{t}}$, an additional prediction for the LUXQED17 PDF set with $m_{\mathrm{t}}=172.5 \mathrm{GeV}$ is provided.

- A prediction [77] that combines the NNLO QCD calculations with the double resummation of soft and small-mass logarithms to NNLL' accuracy, matched with both the standard soft-gluon resummation at NNLL accuracy and the fixed-order calculation at NNLO accuracy (NNLO+NNLL'). These corrections are expected to affect the high-energy tails of the $t \bar{t}$ differential distributions. The calculation is performed using the NNPDF3.1 PDF set [78], and dynamic renormalisation and factorisation scales $\left(m_{\mathrm{t}} / 2\right.$ for $p_{\mathrm{T}}^{\mathrm{t}}$ and $H_{\mathrm{T}} / 4$ for $\left.m_{\mathrm{t} \overline{\mathrm{t}}}\right)$. Predictions are provided for $m_{\mathrm{t}}$ values of 173.3 and $172.5 \mathrm{GeV}$.

- An approximate next-to-NNLO calculation [22] ( $\left.\mathrm{aN}^{3} \mathrm{LO}\right)$ based on the resummation of soft-gluon contributions in the double-differential cross section at NNLL accuracy in the moment-space approach. The NNPDF3.0 PDF set is used and $m_{\mathrm{t}}$ is set to 
$172.5 \mathrm{GeV}$. The renormalisation and factorisation scales are set to $m_{\mathrm{T}}$ for the $p_{\mathrm{T}}^{\mathrm{t}}$ distribution and $m_{\mathrm{t}}$ for the $y_{\mathrm{t}}$ distribution.

- An approximate NNLO calculation [21] (aNNLO), based on QCD threshold expansions beyond the leading-logarithmic approximation using the CT14nnlo [79] PDF set. The top quark mass and dynamic factorisation and renormalisation scales are set to $m_{\mathrm{t}}=172.5 \mathrm{GeV}$.

The NNLO $+\alpha_{\mathrm{EW}}^{3}$ predictions include uncertainties from variations of the renormalisation and factorisation scales and from the PDFs. The NNLO+NNLL' and aN ${ }^{3} \mathrm{LO}$ predictions include uncertainties from scale variations only. The aNNLO prediction includes uncertainties from the PDFs only.

For the NNLO $+\alpha_{\mathrm{EW}}^{3}$ calculations, predictions for the $p_{\mathrm{T}}^{\mathrm{t}}, p_{\mathrm{T}}^{\overline{\mathrm{t}}}, y_{\mathrm{t}}, y_{\overline{\mathrm{t}}}, p_{\mathrm{T}}^{\mathrm{t} \overline{\mathrm{t}}}, m_{\mathrm{t} \overline{\mathrm{t}}}$, and $\Delta|y|(\mathrm{t}, \overline{\mathrm{t}})$ distributions are provided. For the NNLO+NNLL' calculation, predictions for the average of the $p_{\mathrm{T}}^{\mathrm{t}}$ and $p_{\mathrm{T}}^{\overline{\mathrm{t}}}$ distributions and for the $m_{\mathrm{t} \overline{\mathrm{t}}}$ distribution are provided. For the $\mathrm{aN}^{3} \mathrm{LO}$ calculation, predictions for the $p_{\mathrm{T}}^{\mathrm{t}}$ and $y_{\mathrm{t}}$ distributions are provided. For the aNNLO calculation, predictions for the $p_{\mathrm{T}}^{\mathrm{t}}$ distribution and the average of the $y_{\mathrm{t}}$ and $y_{\overline{\mathrm{t}}}$ distributions are provided. Since the differences between the averaged predictions and the corresponding separate predictions for top quark and antiquark are expected to be small, the averaged predictions are compared to the top quark distributions in data.

\subsection{Commentary on results}

All measured differential cross sections, along with figures giving the contribution of each source of uncertainty to the total uncertainty for selected normalised parton-level measurements, are shown in figures 3-32. Absolute and normalised results at the particle and parton levels for a given observable are grouped together in each figure. Within the figure, the upper row corresponds to the parton-level measurement in the full phase space, and the lower row to the particle-level measurement in the fiducial phase space. The left column corresponds to the absolute measurement and the right column to the normalised measurement. In each plot the top panel shows the measured differential cross section with the predictions overlayed and the bottom panel shows the ratios of the predictions to the measured distribution and the statistical and total uncertainties in the measured distribution. When predictions with beyond-NLO precision are available, additional figures with comparisons of these predictions to data are included. In addition, the numerical values of the measured differential cross sections in each bin and associated uncertainties for all observables are tabulated in tables 1-14 in appendix A for parton level and in tables 15-47 in appendix B for particle level.

The results for observables measured only at particle level are shown in figures 3351. Within each figure, the left plot corresponds to the absolute measurements, and the right plot to the normalised measurements. The measurements of the kinematic properties of the leptons and $b$ jets probe the modelling of the $t \bar{t}$ production and top quark decay. Because of the excellent lepton energy resolution, the measurements of the lepton kinematic observables are particularly precise. The measurement of $\Delta \phi(\ell, \bar{\ell})$ is used to constrain the 

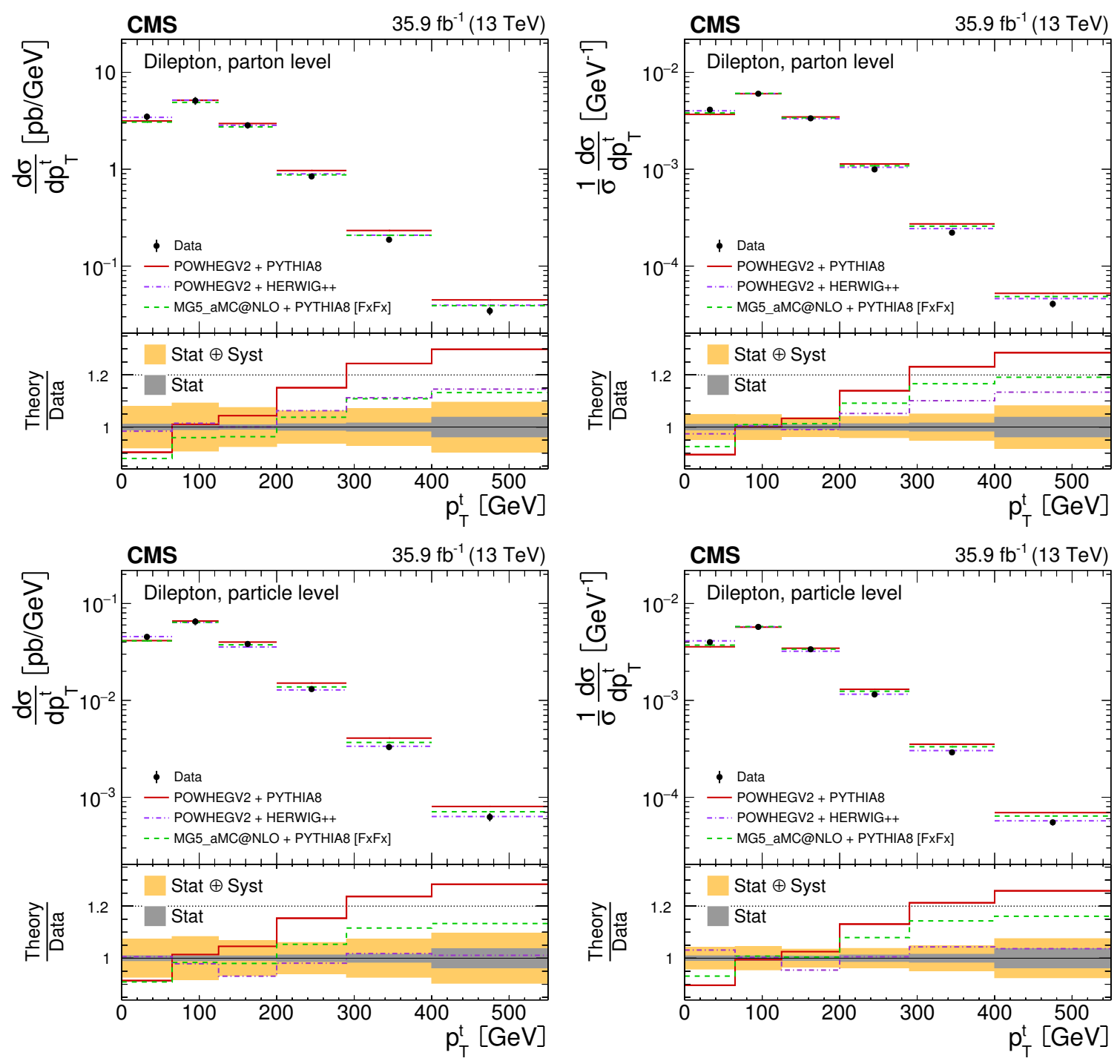

Figure 3. The differential tt production cross sections as a function of $p_{\mathrm{T}}^{\mathrm{t}}$ are shown for the data (points) and the MC predictions (lines). The vertical lines on the points indicate the total uncertainty in the data. The left and right columns correspond to absolute and normalised measurements, respectively. The upper row corresponds to measurements at the parton level in the full phase space and the lower row to the particle level in a fiducial phase space. The lower panel in each plot shows the ratios of the theoretical predictions to the data. The dark and light bands show the relative statistical and total uncertainties in the data, respectively. 

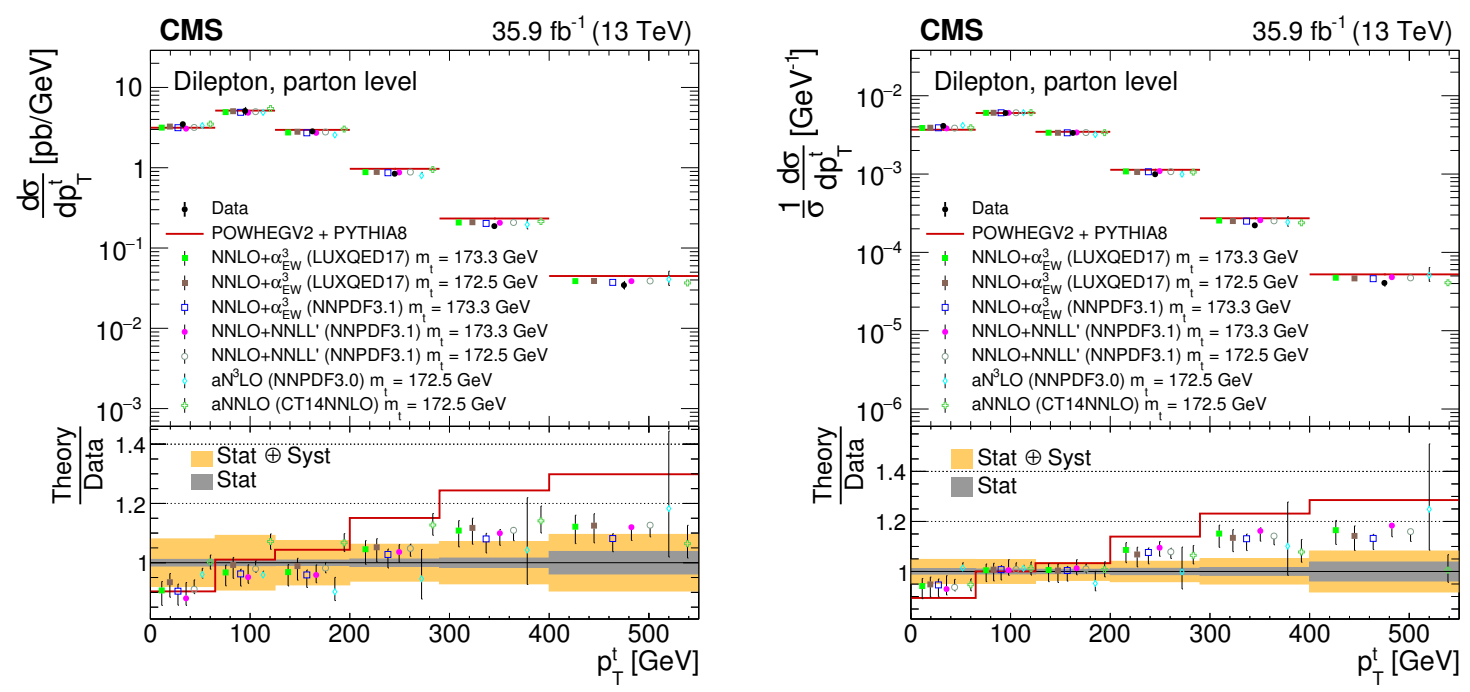

Figure 4. The differential t $\bar{t}$ production cross sections at the parton level in the full phase space as a function of $p_{\mathrm{T}}^{\mathrm{t}}$ are shown for the data (filled circles), the theoretical predictions with beyond-NLO precision (other points) and the prediction from POWHEG+PYTHIA (solid line). The vertical lines on the filled circles and other points indicate the total uncertainty in the data and theoretical predictions, respectively. The left and right plots correspond to absolute and normalised measurements, respectively. The lower panel in each plot shows the ratios of the theoretical predictions to the data. The dark and light bands show the relative statistical and total uncertainties in the data, respectively.

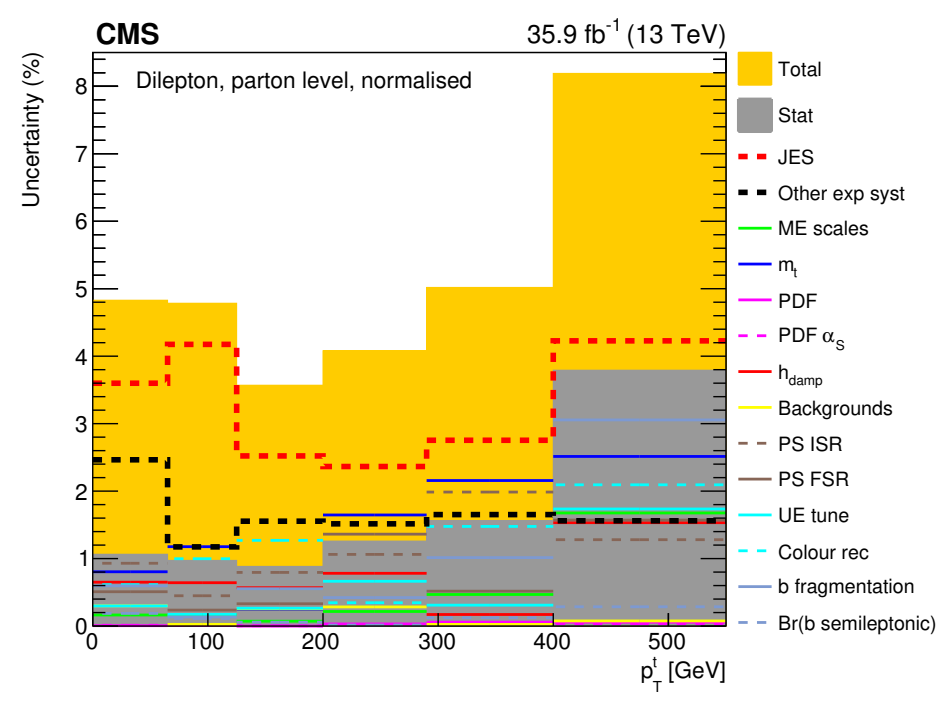

Figure 5. The contributions of each source of systematic uncertainty in each bin is shown for the measurement of the normalised $t \bar{t}$ production cross sections as a function of $p_{\mathrm{T}}^{\mathrm{t}}$. The sources affecting the JES are added in quadrature and shown as a single component. Additional experimental systematic uncertainties are also added in quadrature and shown as a single component. Contributions from theoretical uncertainties are shown separately. The statistical and total uncertainties, corresponding to the quadrature addition of statistical and systematic uncertainties, are shown by the dark and light filled histograms, respectively. 

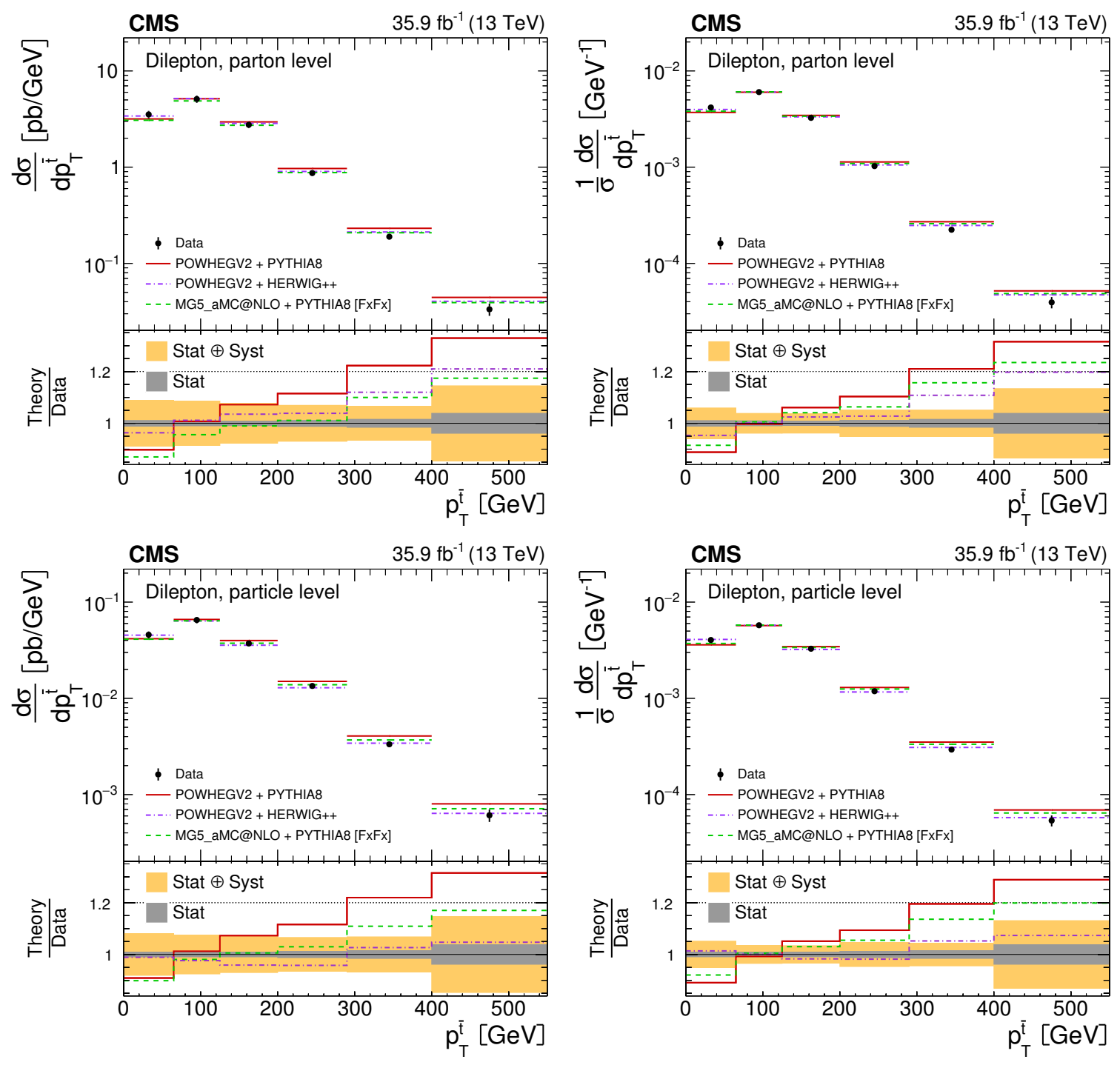

Figure 6. The differential t $t \overline{\mathrm{t}}$ production cross sections as a function of $p_{\mathrm{T}}^{\overline{\mathrm{t}}}$ are shown for the data (points) and the MC predictions (lines). The vertical lines on the points indicate the total uncertainty in the data. The left and right columns correspond to absolute and normalised measurements, respectively. The upper row corresponds to measurements at the parton level in the full phase space and the lower row to the particle level in a fiducial phase space. The lower panel in each plot shows the ratios of the theoretical predictions to the data. The dark and light bands show the relative statistical and total uncertainties in the data, respectively. 

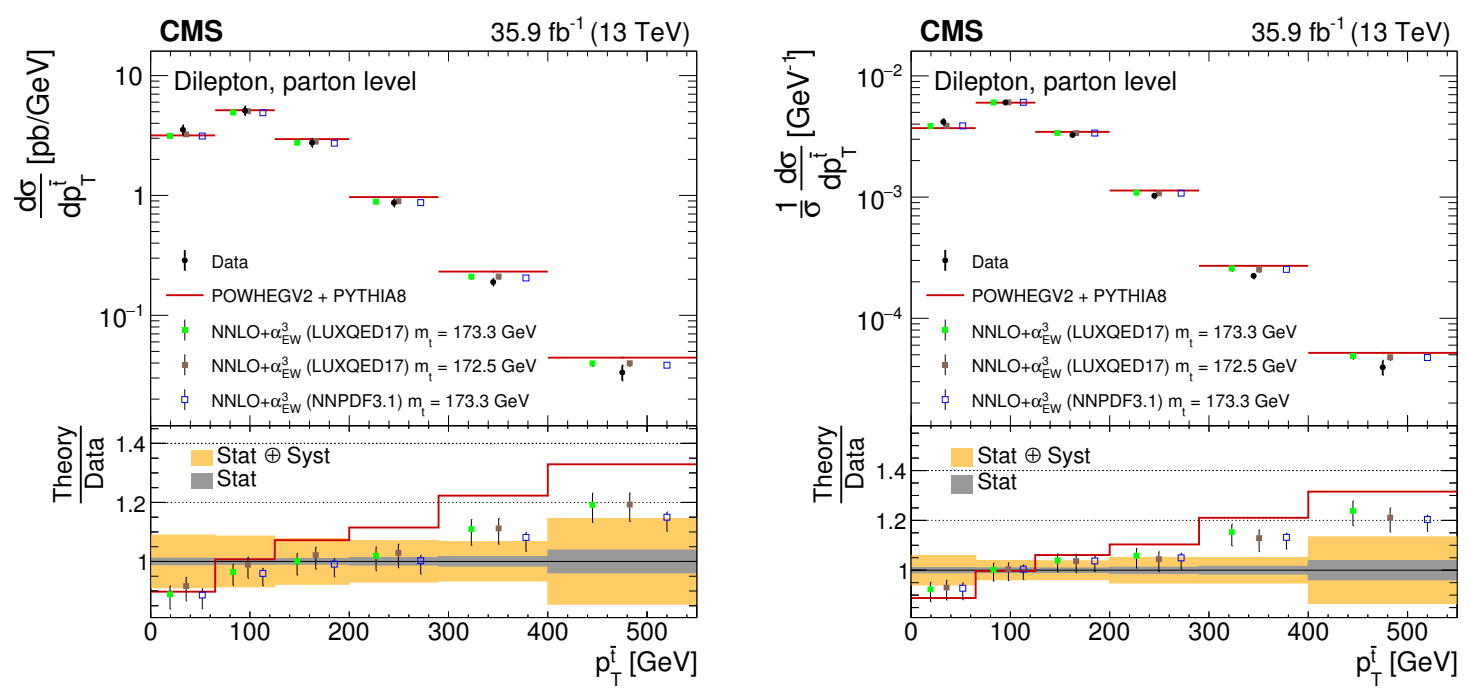

Figure 7. The differential t $\bar{t}$ production cross sections at the parton level in the full phase space as a function of $p_{\mathrm{T}}^{\overline{\mathrm{t}}}$ are shown for the data (filled circles), the theoretical predictions with beyond-NLO precision (other points) and the prediction from POWHEG+PYTHIA (solid line). The vertical lines on the filled circles and other points indicate the total uncertainty in the data and theoretical predictions, respectively. The left and right plots correspond to absolute and normalised measurements, respectively. The lower panel in each plot shows the ratios of the theoretical predictions to the data. The dark and light bands show the relative statistical and total uncertainties in the data, respectively.

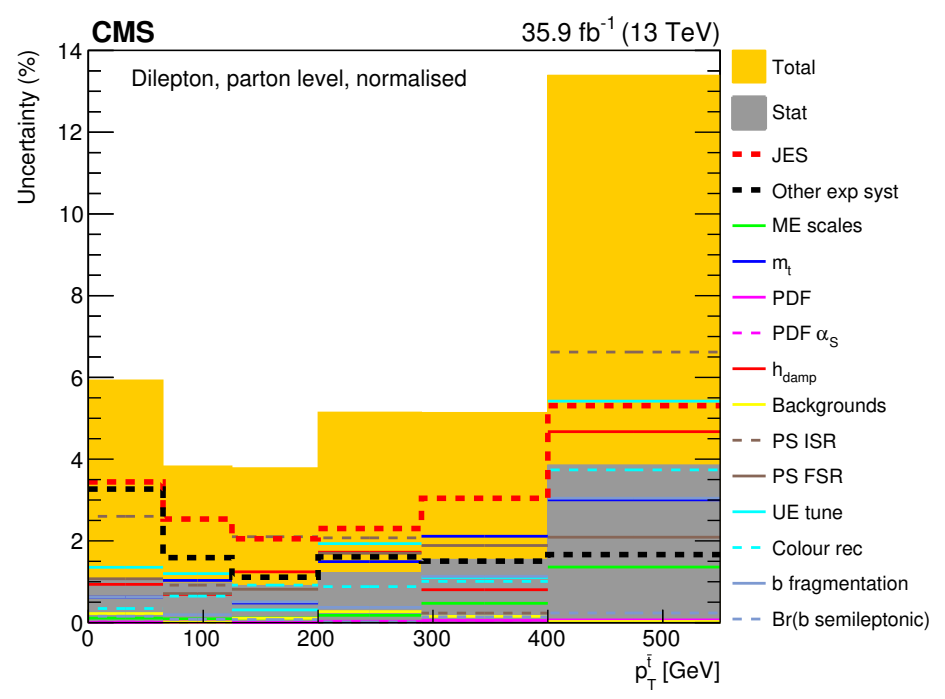

Figure 8. The contributions of each source of systematic uncertainty to the total systematic uncertainty in each bin is shown for the measurement of the normalised t $\mathrm{t}$ production cross sections as a function of $p_{\mathrm{T}}^{\bar{t}}$. The sources affecting the JES are added in quadrature and shown as a single component. Additional experimental systematic uncertainties are also added in quadrature and shown as a single component. Contributions from theoretical uncertainties are shown separately. The statistical and total uncertainties, corresponding to the quadrature addition of statistical and systematic uncertainties, are shown by the dark and light filled histograms, respectively. 

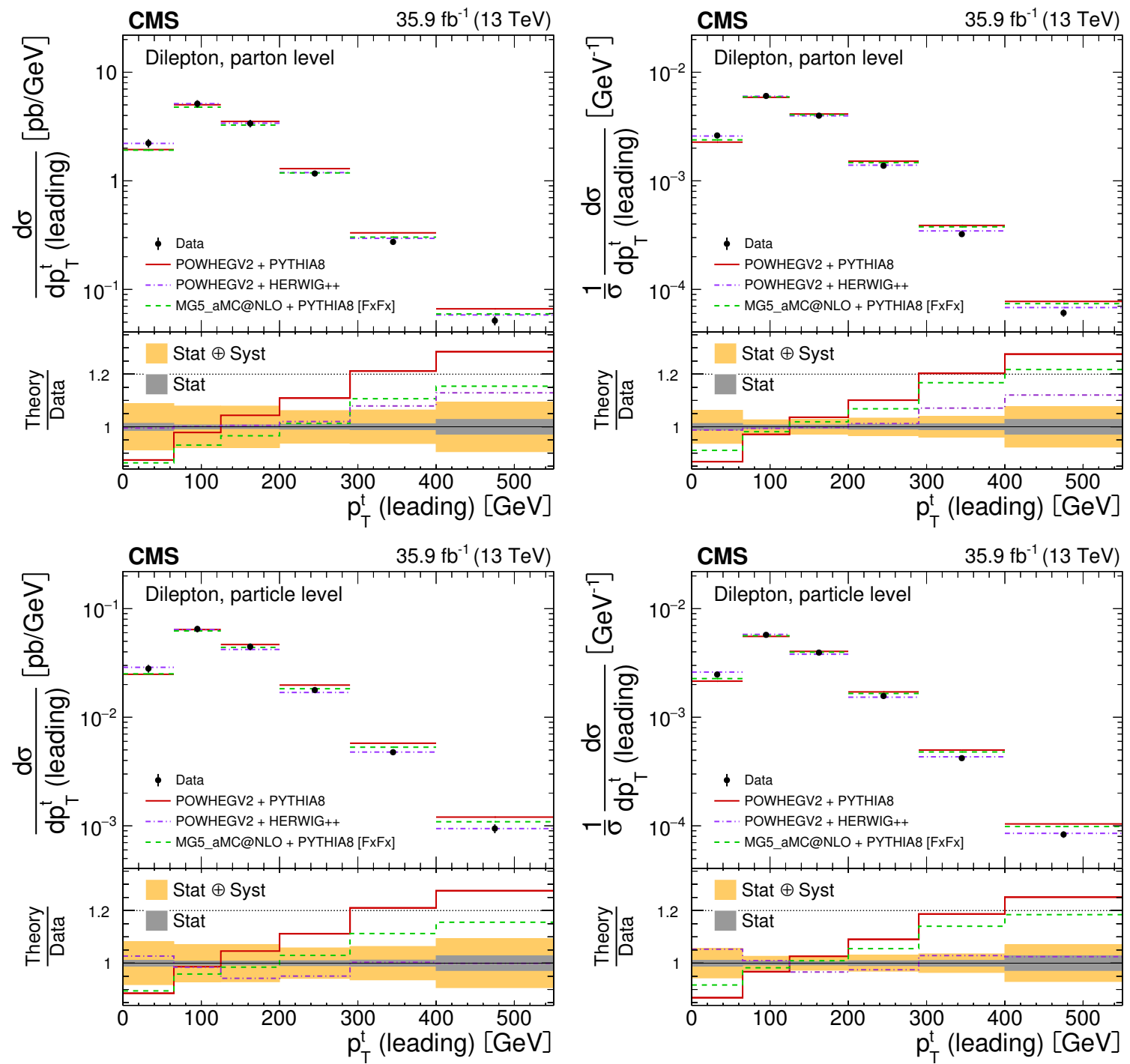

Figure 9. The differential t $\overline{\mathrm{t}}$ production cross sections as a function of $p_{\mathrm{T}}^{\mathrm{t}}$ (leading) are shown for the data (points) and the MC predictions (lines). The vertical lines on the points indicate the total uncertainty in the data. The left and right columns correspond to absolute and normalised measurements, respectively. The upper row corresponds to measurements at the parton level in the full phase space and the lower row to the particle level in a fiducial phase space. The lower panel in each plot shows the ratios of the theoretical predictions to the data. The dark and light bands show the relative statistical and total uncertainties in the data, respectively. 

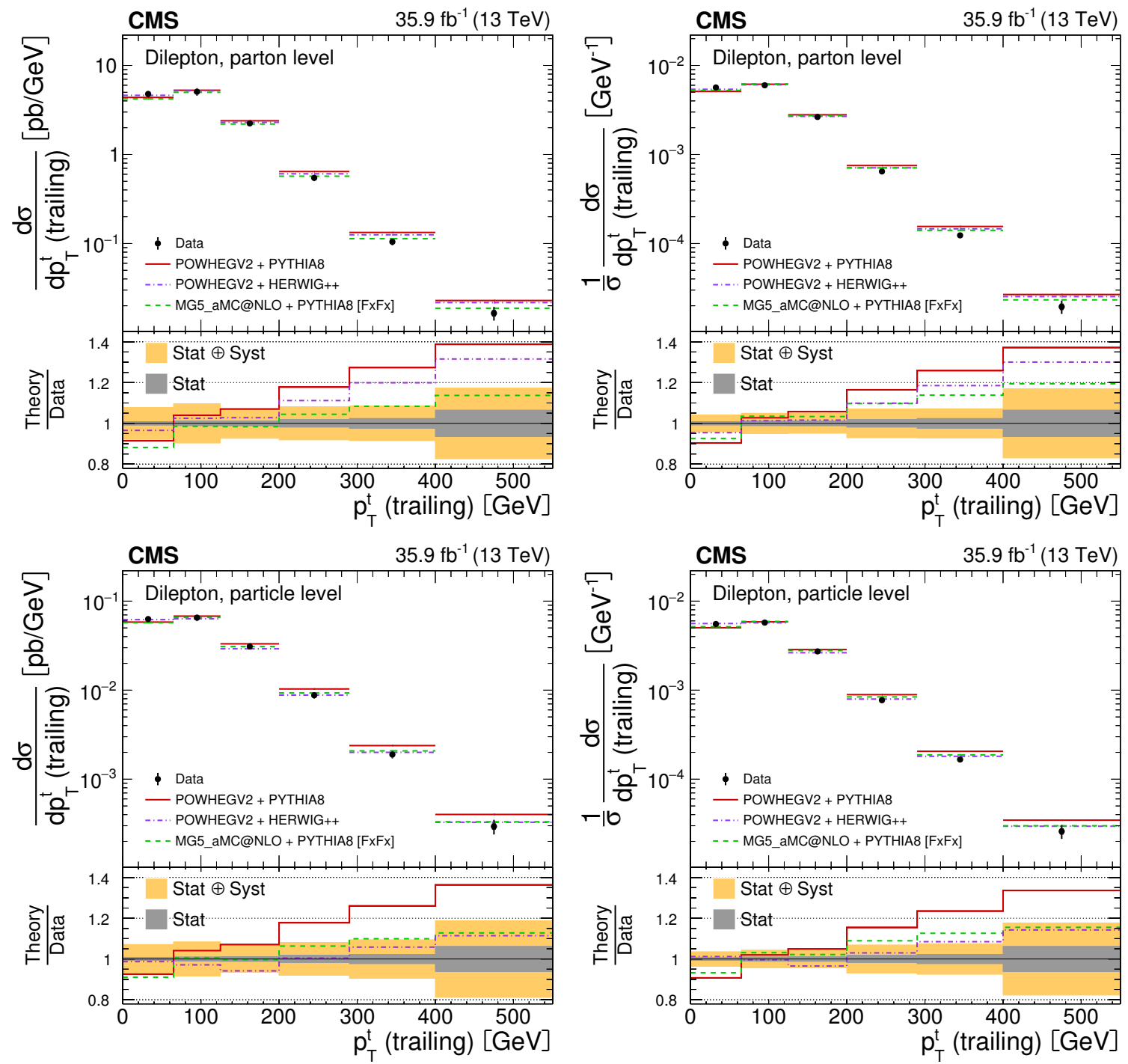

Figure 10. The differential t $\overline{\mathrm{t}}$ production cross sections as a function of $p_{\mathrm{T}}^{\mathrm{t}}$ (trailing) are shown for the data (points) and the MC predictions (lines). The vertical lines on the points indicate the total uncertainty in the data. The left and right columns correspond to absolute and normalised measurements, respectively. The upper row corresponds to measurements at the parton level in the full phase space and the lower row to the particle level in a fiducial phase space. The lower panel in each plot shows the ratios of the theoretical predictions to the data. The dark and light bands show the relative statistical and total uncertainties in the data, respectively. 

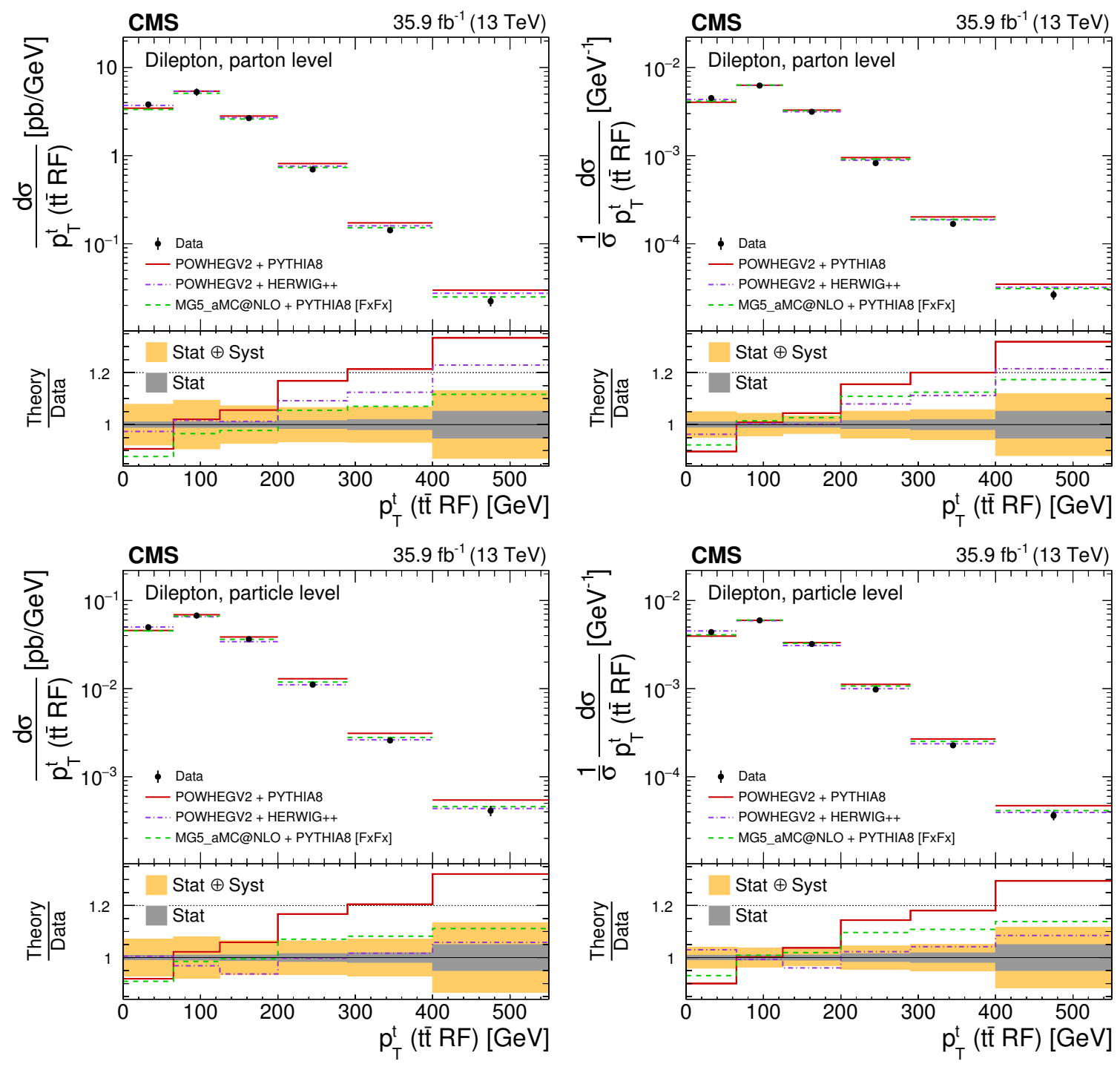

Figure 11. The differential t t production cross sections as a function of $p_{\mathrm{T}}^{\mathrm{t}}(\mathrm{t} \overline{\mathrm{t}} \mathrm{RF})$ are shown for the data (points) and the MC predictions (lines). The vertical lines on the points indicate the total uncertainty in the data. The left and right columns correspond to absolute and normalised measurements, respectively. The upper row corresponds to measurements at the parton level in the full phase space and the lower row to the particle level in a fiducial phase space. The lower panel in each plot shows the ratios of the theoretical predictions to the data. The dark and light bands show the relative statistical and total uncertainties in the data, respectively. 

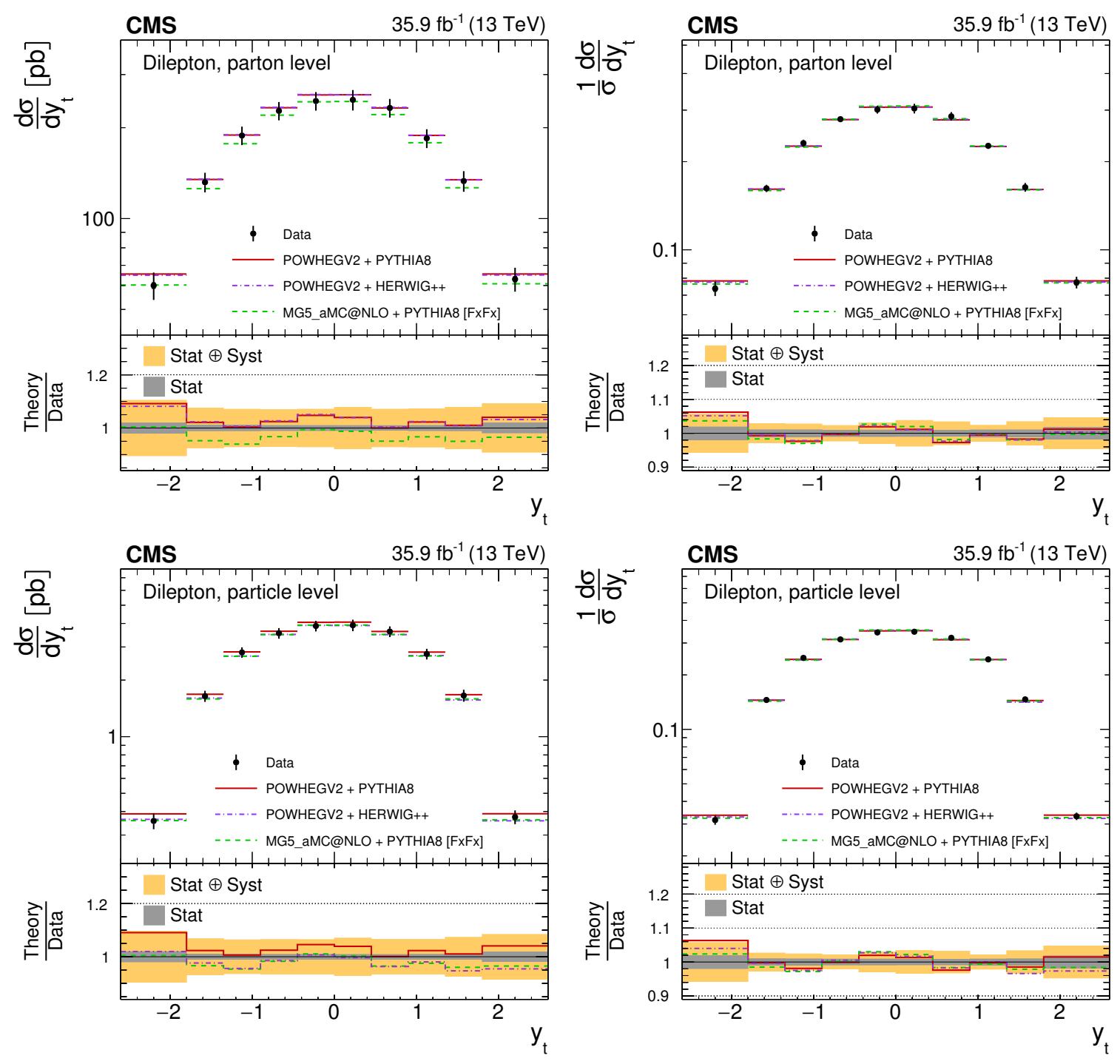

Figure 12. The differential t $\bar{t}$ production cross sections as a function of $y_{\mathrm{t}}$ are shown for the data (points) and the MC predictions (lines). The vertical lines on the points indicate the total uncertainty in the data. The left and right columns correspond to absolute and normalised measurements, respectively. The upper row corresponds to measurements at the parton level in the full phase space and the lower row to the particle level in a fiducial phase space. The lower panel in each plot shows the ratios of the theoretical predictions to the data. The dark and light bands show the relative statistical and total uncertainties in the data, respectively. 

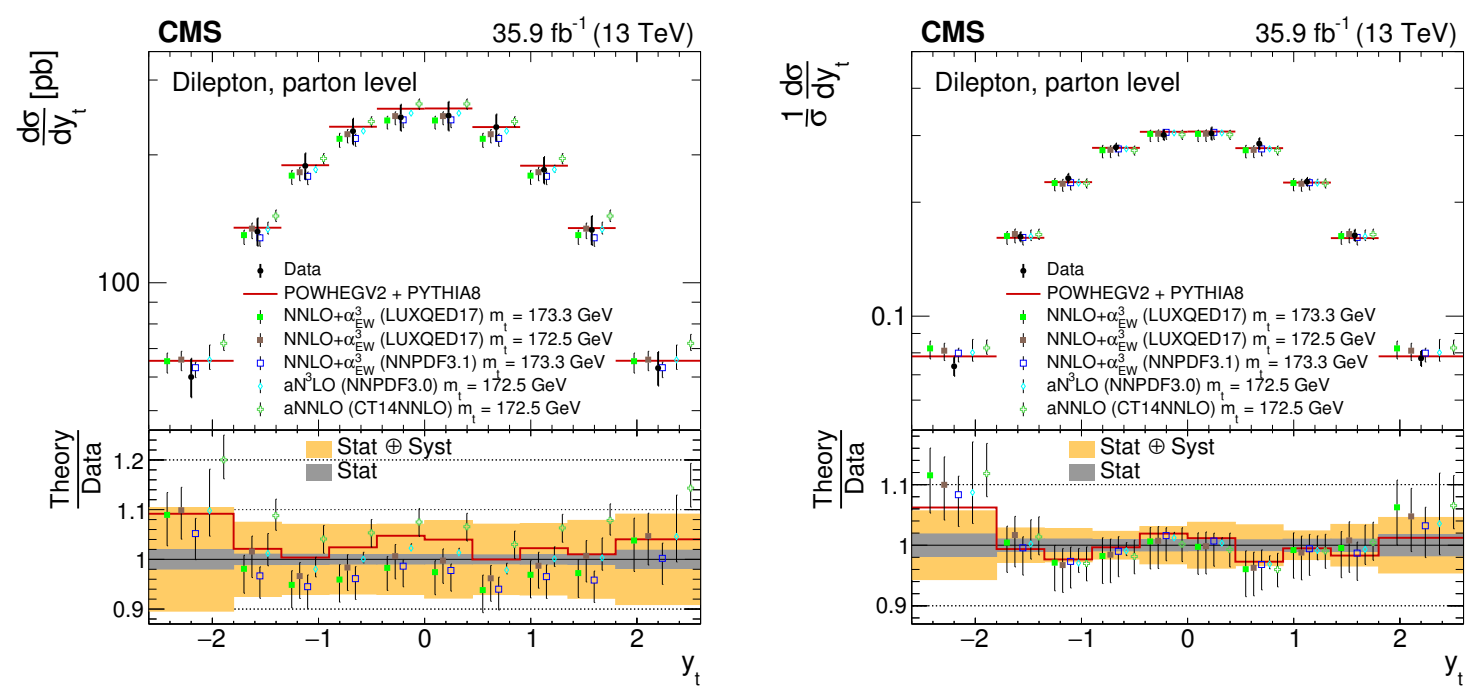

Figure 13. The differential te production cross sections at the parton level in the full phase space as a function of $y_{\mathrm{t}}$ are shown for the data (filled circles), the theoretical predictions with beyondNLO precision (other points) and the prediction from POWHEG+PYTHIA (solid line). The vertical lines on the filled circles and other points indicate the total uncertainty in the data and theoretical predictions, respectively. The left and right plots correspond to absolute and normalised measurements, respectively. The lower panel in each plot shows the ratios of the theoretical predictions to the data. The dark and light bands show the relative statistical and total uncertainties in the data, respectively.

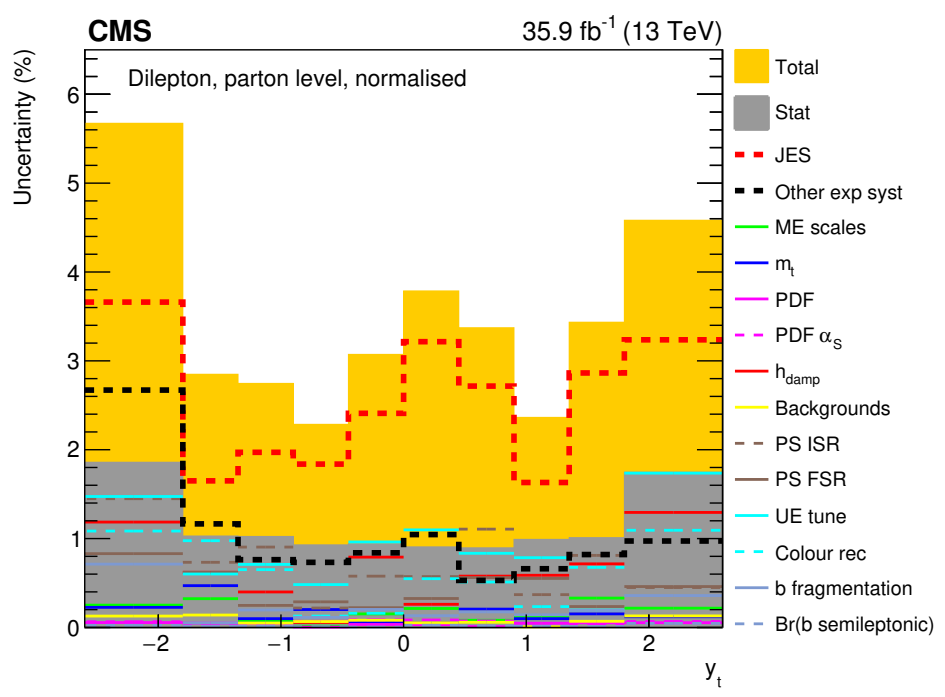

Figure 14. The contributions of each source of systematic uncertainty to the total systematic uncertainty in each bin is shown for the measurement of the normalised t $\bar{t}$ production cross sections as a function of $y_{\mathrm{t}}$. The sources affecting the JES are added in quadrature and shown as a single component. Additional experimental systematic uncertainties are also added in quadrature and shown as a single component. Contributions from theoretical uncertainties are shown separately. The statistical and total uncertainties, corresponding to the quadrature addition of statistical and systematic uncertainties, are shown by the dark and light filled histograms, respectively. 

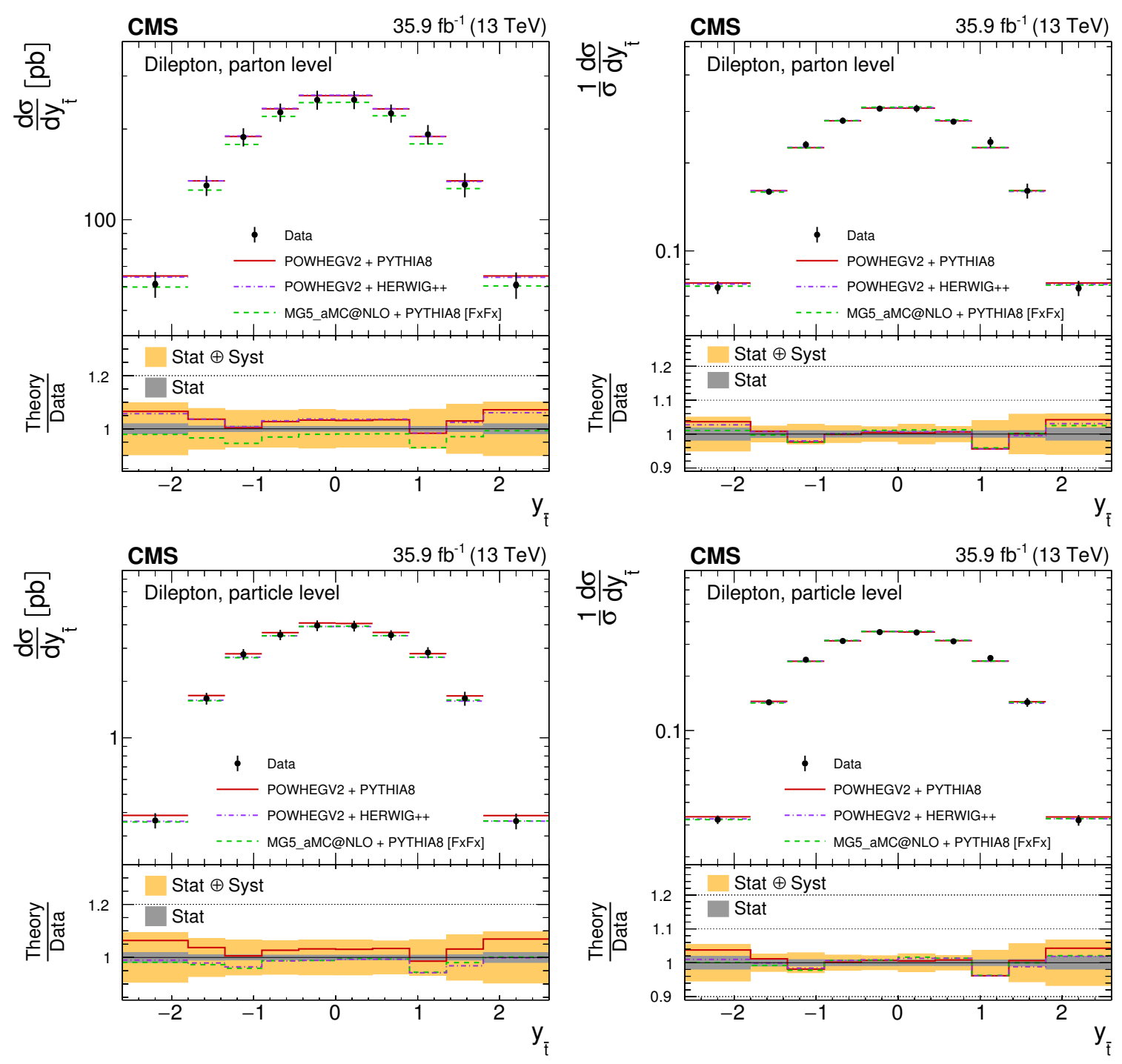

Figure 15. The differential t $\overline{\mathrm{t}}$ production cross sections as a function of $y_{\overline{\mathrm{t}}}$ are shown for the data (points) and the MC predictions (lines). The vertical lines on the points indicate the total uncertainty in the data. The left and right columns correspond to absolute and normalised measurements, respectively. The upper row corresponds to measurements at the parton level in the full phase space and the lower row to the particle level in a fiducial phase space. The lower panel in each plot shows the ratios of the theoretical predictions to the data. The dark and light bands show the relative statistical and total uncertainties in the data, respectively. 

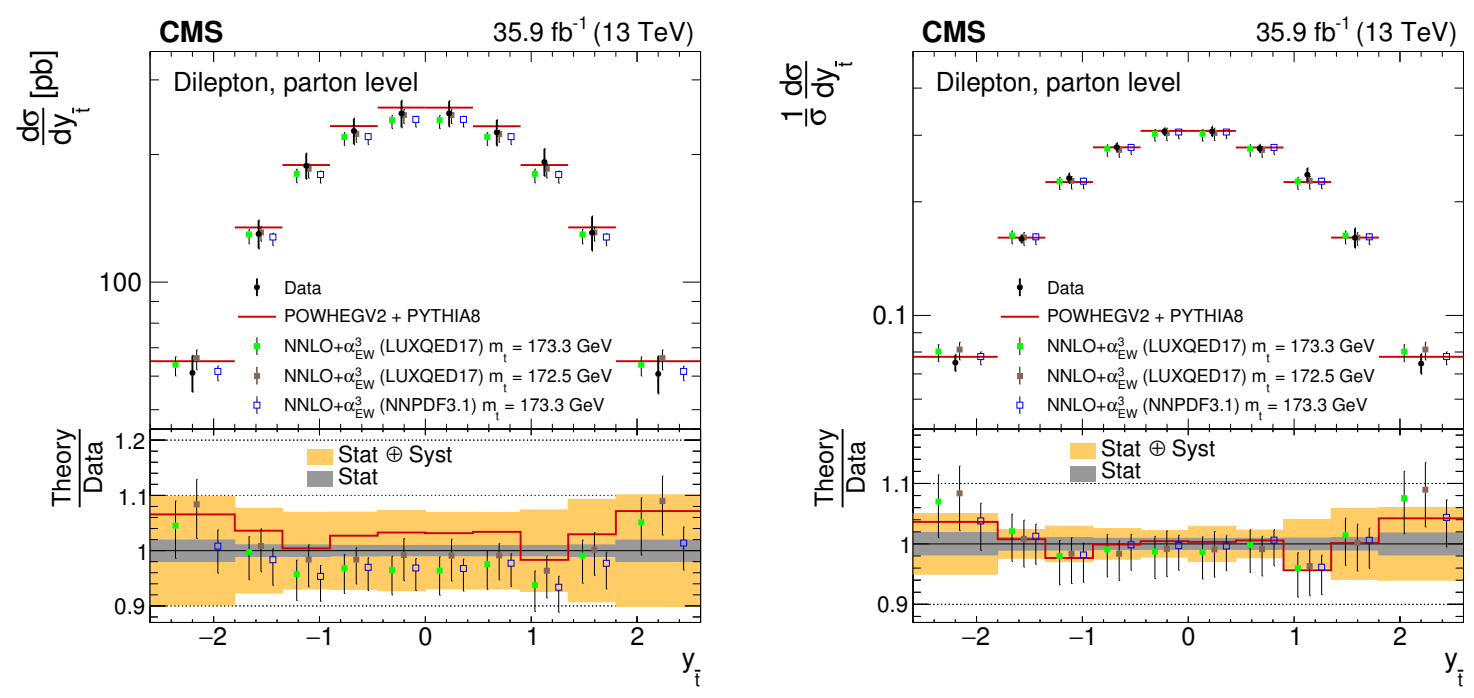

Figure 16. The differential te production cross sections at the parton level in the full phase space as a function of $y_{\bar{t}}$ are shown for the data (filled circles), the theoretical predictions with beyondNLO precision (other points) and the prediction from POWHEG+PYTHIA (solid line). The vertical lines on the filled circles and other points indicate the total uncertainty in the data and theoretical predictions, respectively. The left and right plots correspond to absolute and normalised measurements, respectively. The lower panel in each plot shows the ratios of the theoretical predictions to the data. The dark and light bands show the relative statistical and total uncertainties in the data, respectively.

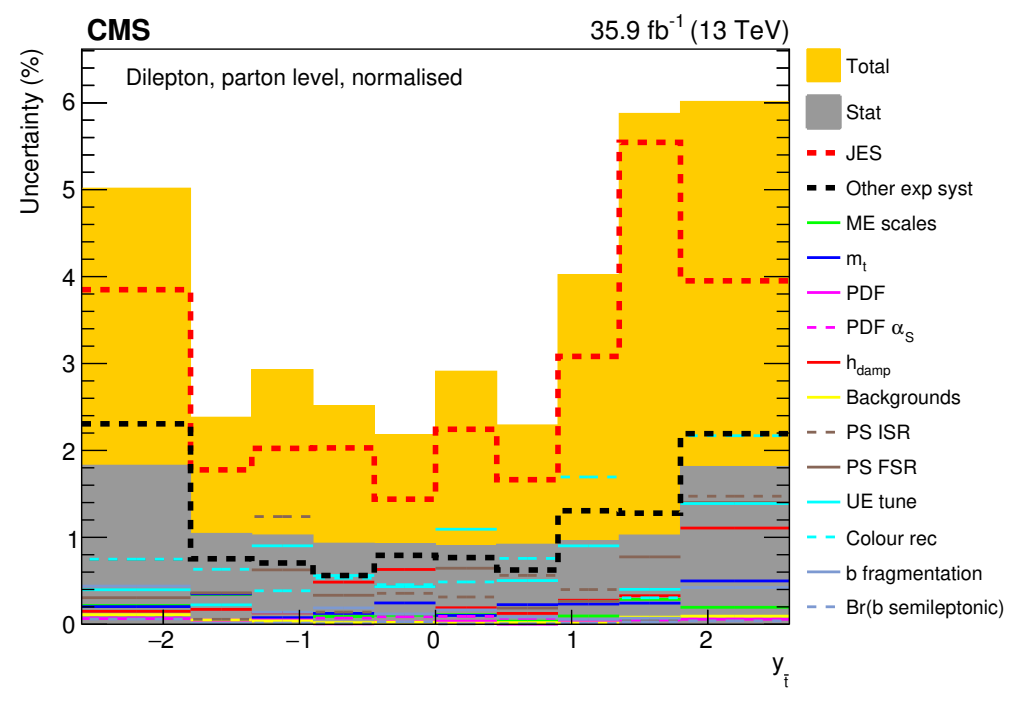

Figure 17. The contributions of each source of systematic uncertainty to the total systematic uncertainty in each bin is shown for the measurement of the normalised t $t \bar{t}$ production cross sections as a function of $y_{\overline{\mathrm{t}}}$. The sources affecting the JES are added in quadrature and shown as a single component. Additional experimental systematic uncertainties are also added in quadrature and shown as a single component. Contributions from theoretical uncertainties are shown separately. The statistical and total uncertainties, corresponding to the quadrature addition of statistical and systematic uncertainties, are shown by the dark and light filled histograms, respectively. 

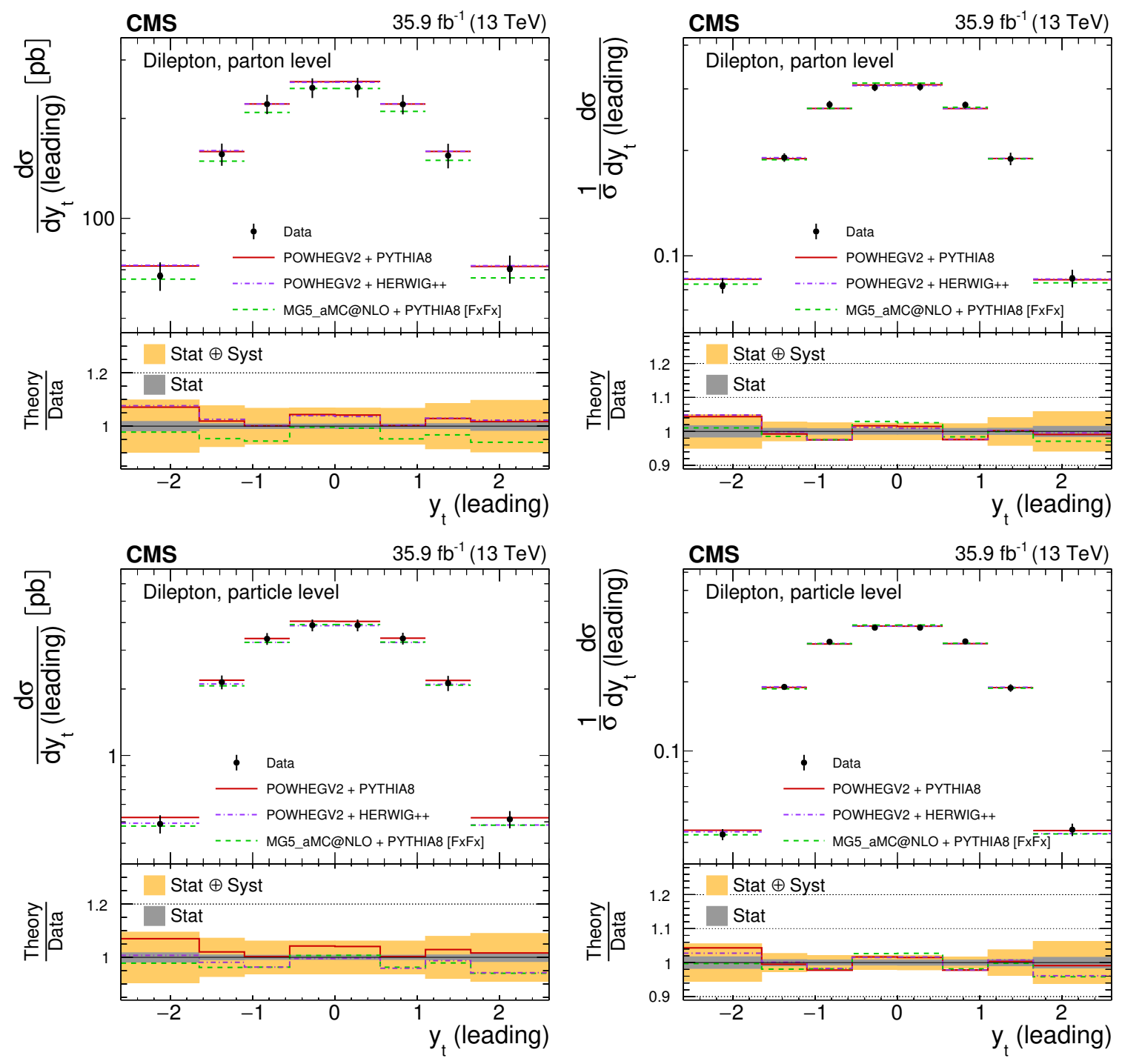

Figure 18. The differential t $\overline{\mathrm{t}}$ production cross sections as a function of $y_{\mathrm{t}}$ (leading) are shown for the data (points) and the MC predictions (lines). The vertical lines on the points indicate the total uncertainty in the data. The left and right columns correspond to absolute and normalised measurements, respectively. The upper row corresponds to measurements at the parton level in the full phase space and the lower row to the particle level in a fiducial phase space. The lower panel in each plot shows the ratios of the theoretical predictions to the data. The dark and light bands show the relative statistical and total uncertainties in the data, respectively. 

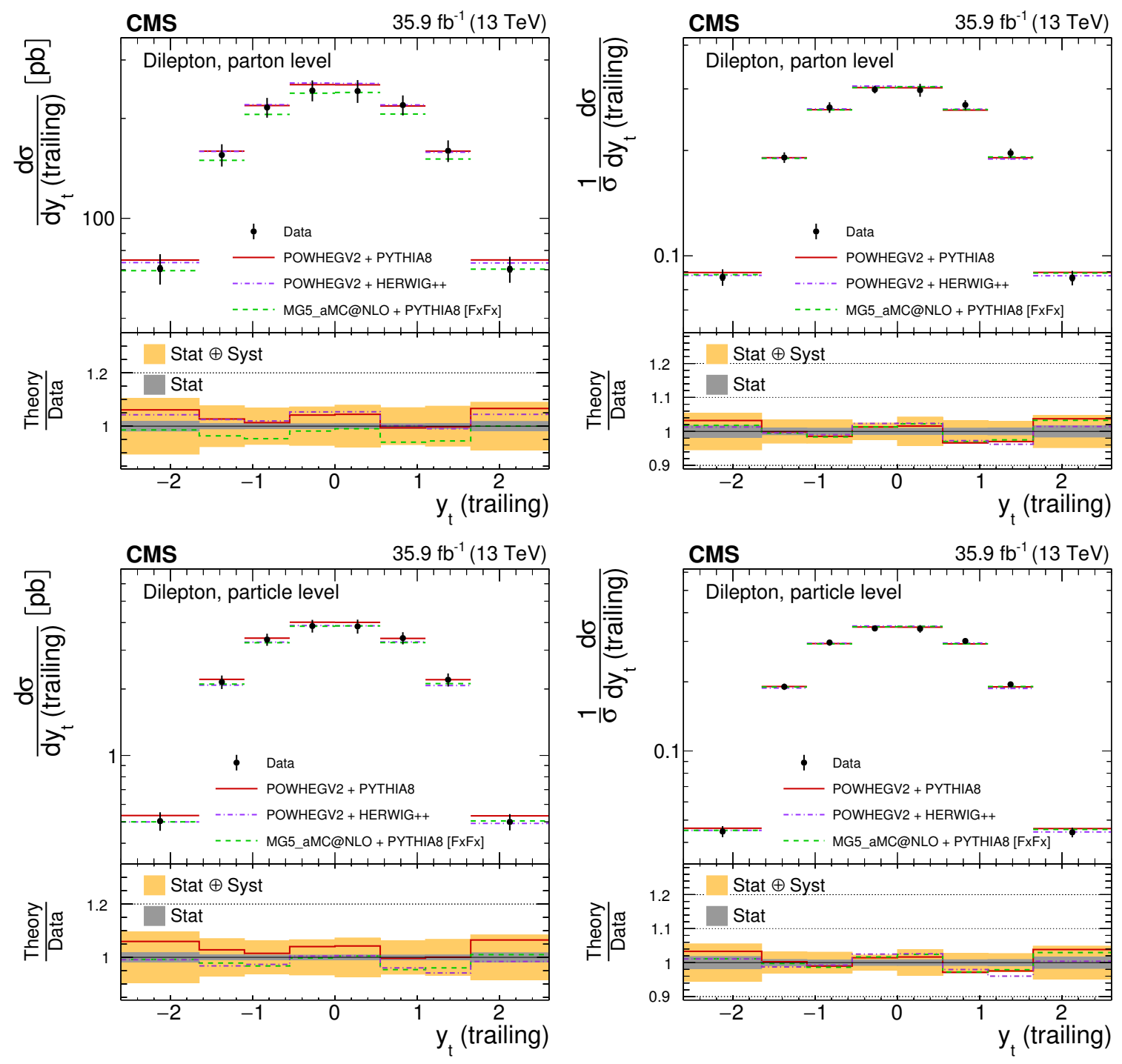

Figure 19. The differential $\mathrm{t} \overline{\mathrm{t}}$ production cross sections as a function of $y_{\mathrm{t}}$ (trailing) are shown for the data (points) and the MC predictions (lines). The vertical lines on the points indicate the total uncertainty in the data. The left and right columns correspond to absolute and normalised measurements, respectively. The upper row corresponds to measurements at the parton level in the full phase space and the lower row to the particle level in a fiducial phase space. The lower panel in each plot shows the ratios of the theoretical predictions to the data. The dark and light bands show the relative statistical and total uncertainties in the data, respectively. 

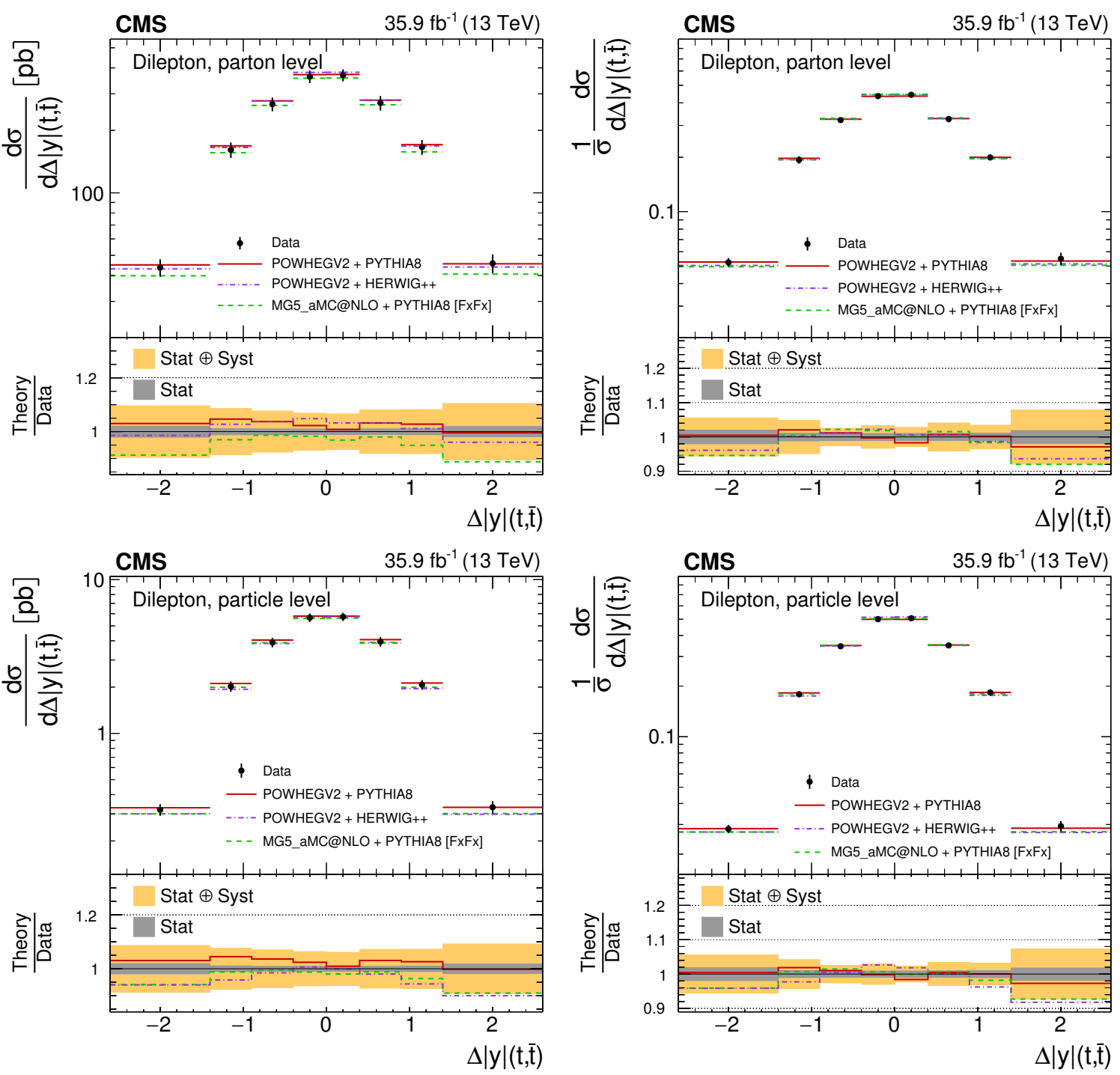

Figure 20. The differential t $\overline{\mathrm{t}}$ production cross sections as a function of $\Delta|y|(\mathrm{t}, \overline{\mathrm{t}})$ are shown for the data (points) and the MC predictions (lines). The vertical lines on the points indicate the total uncertainty in the data. The left and right columns correspond to absolute and normalised measurements, respectively. The upper row corresponds to measurements at the parton level in the full phase space and the lower row to the particle level in a fiducial phase space. The lower panel in each plot shows the ratios of the theoretical predictions to the data. The dark and light bands show the relative statistical and total uncertainties in the data, respectively. 

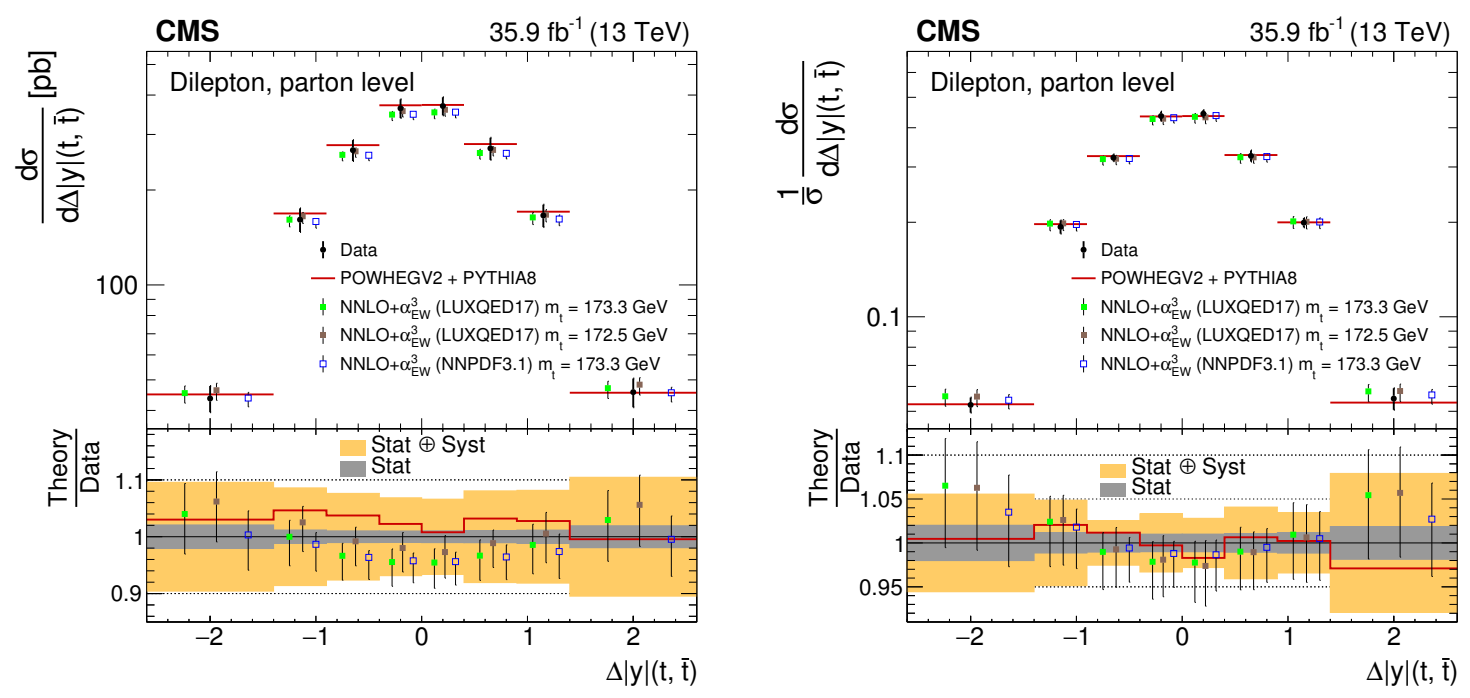

Figure 21. The differential te production cross sections at the parton level in the full phase space as a function of $\Delta|y|(t, \overline{\mathrm{t}})$ are shown for the data (filled circles), the theoretical predictions with beyond-NLO precision (other points) and the prediction from POWHEG+PYTHIA (solid line). The vertical lines on the filled circles and other points indicate the total uncertainty in the data and theoretical predictions, respectively. The left and right plots correspond to absolute and normalised measurements, respectively. The lower panel in each plot shows the ratios of the theoretical predictions to the data. The dark and light bands show the relative statistical and total uncertainties in the data, respectively.

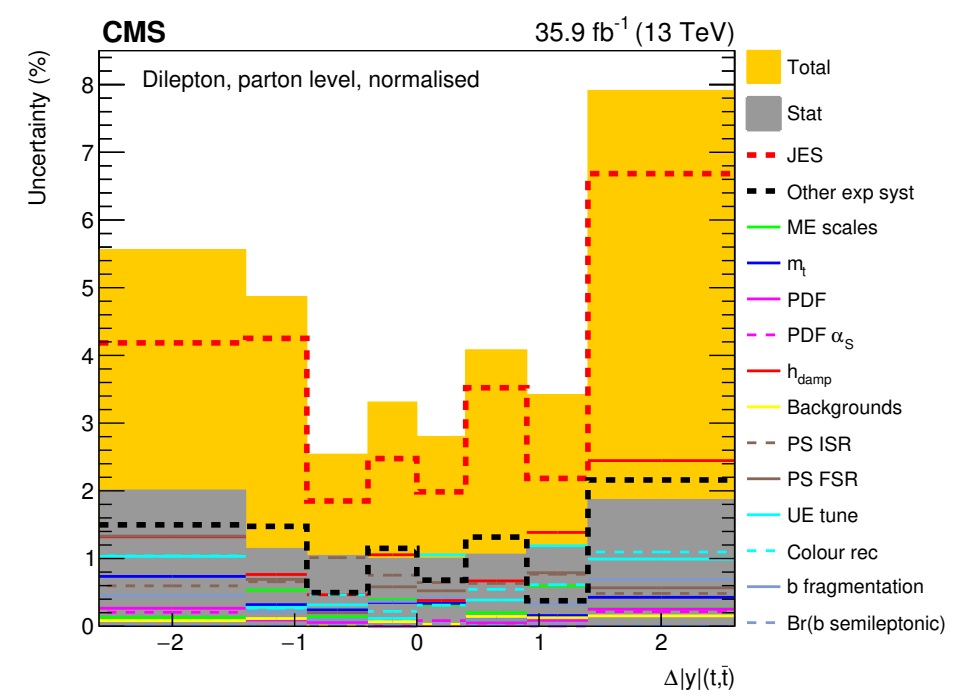

Figure 22. The contributions of each source of systematic uncertainty to the total systematic uncertainty in each bin is shown for the measurement of the normalised t $\bar{t}$ production cross sections as a function of $\Delta|y|(\mathrm{t}, \overline{\mathrm{t}})$. The sources affecting the JES are added in quadrature and shown as a single component. Additional experimental systematic uncertainties are also added in quadrature and shown as a single component. Contributions from theoretical uncertainties are shown separately. The statistical and total uncertainties, corresponding to the quadrature addition of statistical and systematic uncertainties, are shown by the dark and light filled histograms, respectively. 

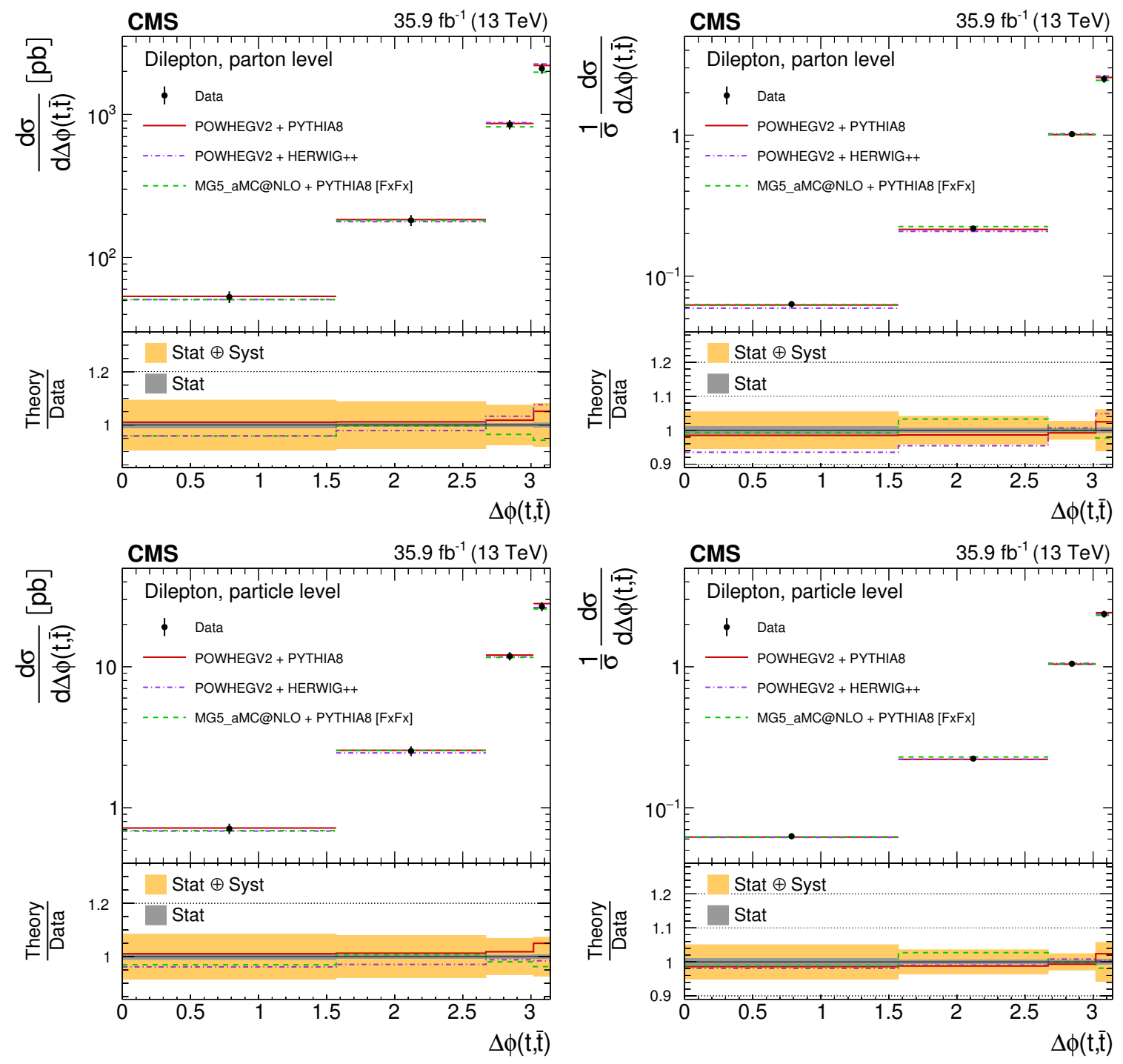

Figure 23. The differential t $\mathrm{t}$ production cross sections as a function of $\Delta \phi(\mathrm{t}, \overline{\mathrm{t}})$ are shown for the data (points) and the MC predictions (lines). The vertical lines on the points indicate the total uncertainty in the data. The left and right columns correspond to absolute and normalised measurements, respectively. The upper row corresponds to measurements at the parton level in the full phase space and the lower row to the particle level in a fiducial phase space. The lower panel in each plot shows the ratios of the theoretical predictions to the data. The dark and light bands show the relative statistical and total uncertainties in the data, respectively. 

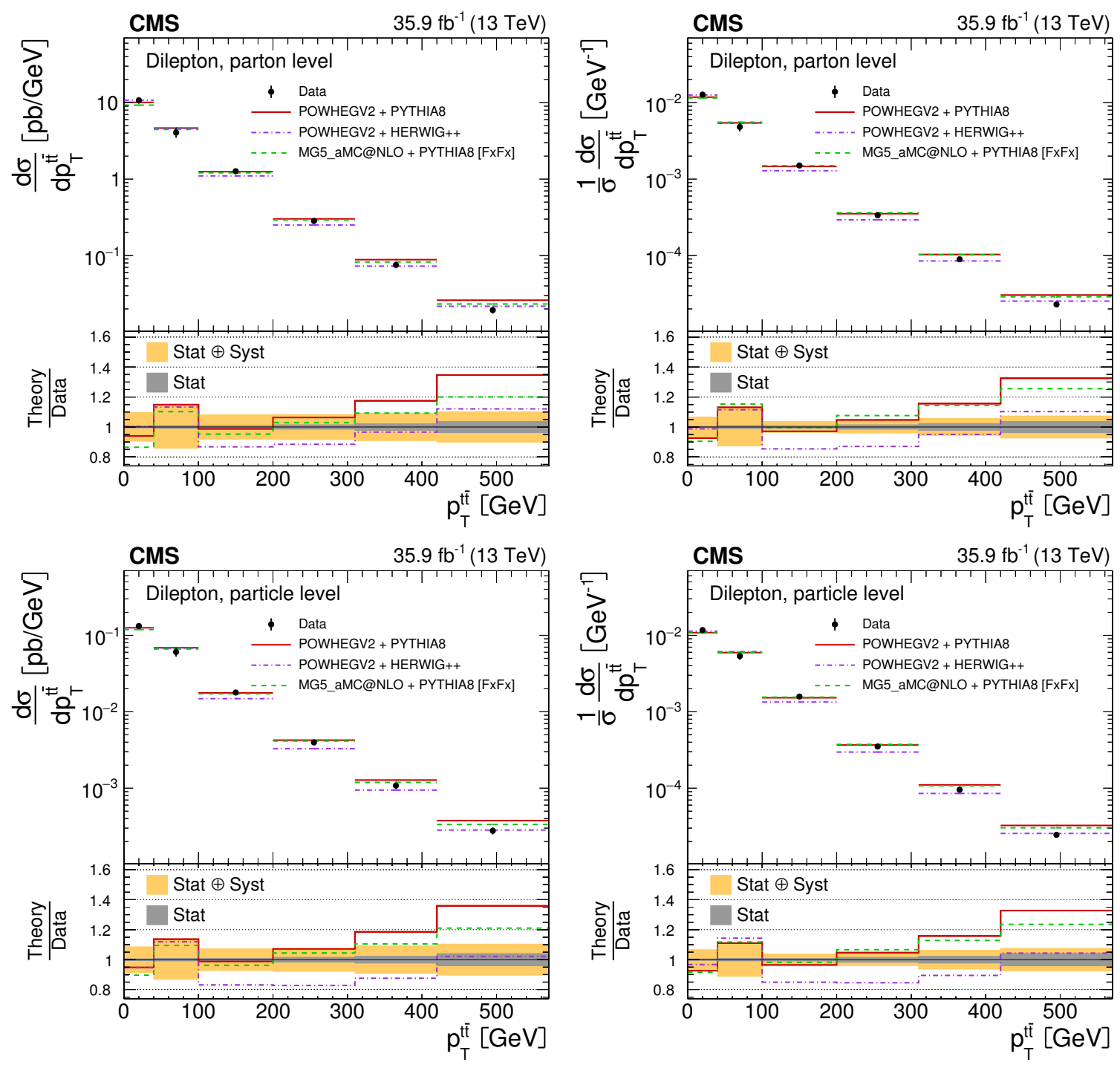

Figure 24. The differential $\mathrm{t} \overline{\mathrm{t}}$ production cross sections as a function of $p_{\mathrm{T}}^{\mathrm{t} \overline{\mathrm{t}}}$ are shown for the data (points) and the MC predictions (lines). The vertical lines on the points indicate the total uncertainty in the data. The left and right columns correspond to absolute and normalised measurements, respectively. The upper row corresponds to measurements at the parton level in the full phase space and the lower row to the particle level in a fiducial phase space. The lower panel in each plot shows the ratios of the theoretical predictions to the data. The dark and light bands show the relative statistical and total uncertainties in the data, respectively. 

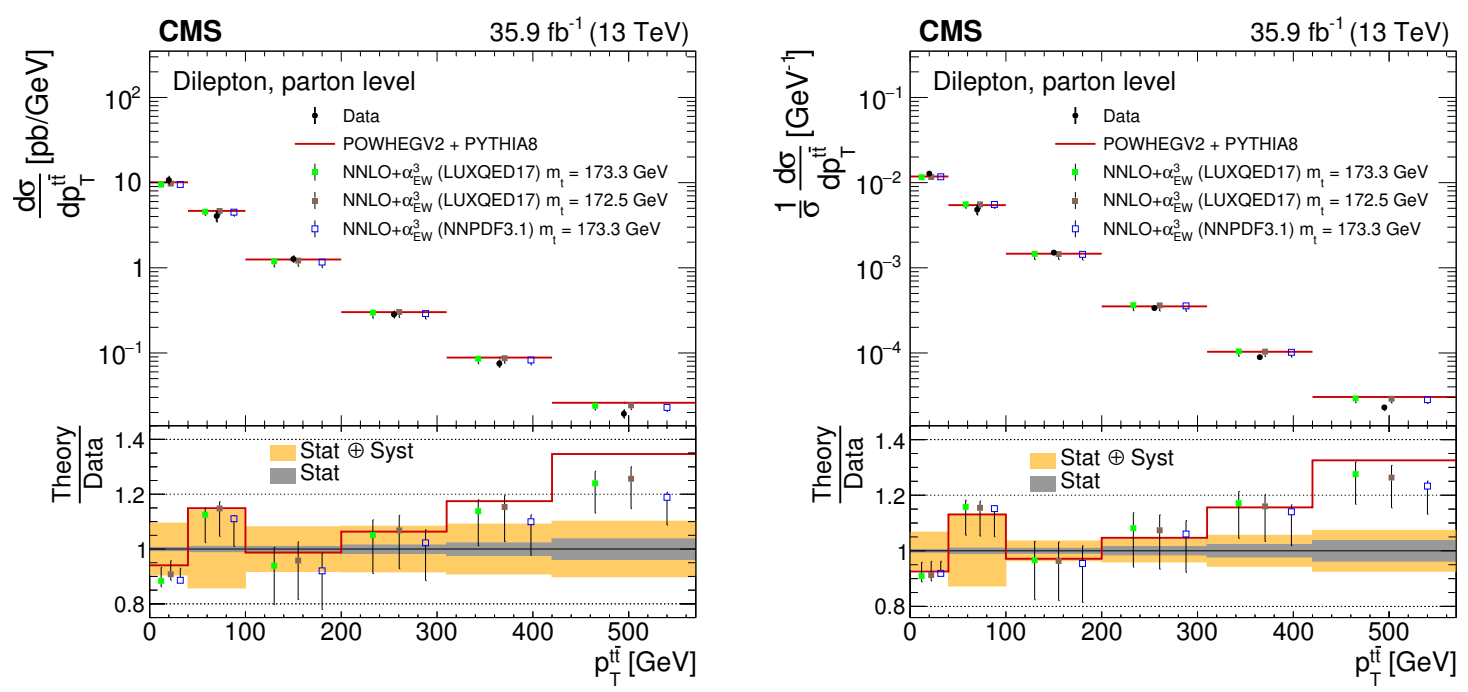

Figure 25. The differential t $\bar{t}$ production cross sections at the parton level in the full phase space as a function of $p_{\mathrm{T}}^{\mathrm{t} \overline{\mathrm{t}}}$ are shown for the data (filled circles), the theoretical predictions with beyondNLO precision (other points) and the prediction from POWHEG+PYTHIA (solid line). The vertical lines on the filled circles and other points indicate the total uncertainty in the data and theoretical predictions, respectively. The left and right plots correspond to absolute and normalised measurements, respectively. The lower panel in each plot shows the ratios of the theoretical predictions to the data. The dark and light bands show the relative statistical and total uncertainties in the data, respectively.

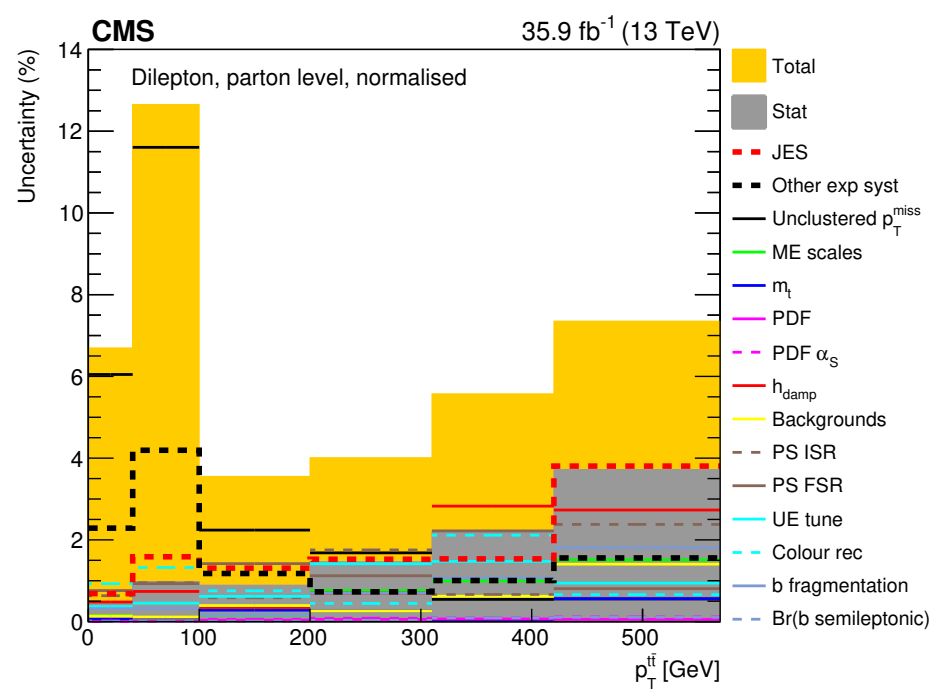

Figure 26. The contributions of each source of systematic uncertainty to the total systematic uncertainty in each bin is shown for the measurement of the normalised $t \bar{t}$ production cross sections as a function of $p_{\mathrm{T}}^{\mathrm{t}}$. The sources affecting the JES are added in quadrature and shown as a single component. As the contribution from unclustered $p_{\mathrm{T}}^{\text {miss }}$ is dominant in lower bins, it is shown separately. Additional experimental systematic uncertainties are added in quadrature and shown as a single component. Contributions from theoretical uncertainties are shown separately. Related theoretical uncertainties are grouped by colour. 

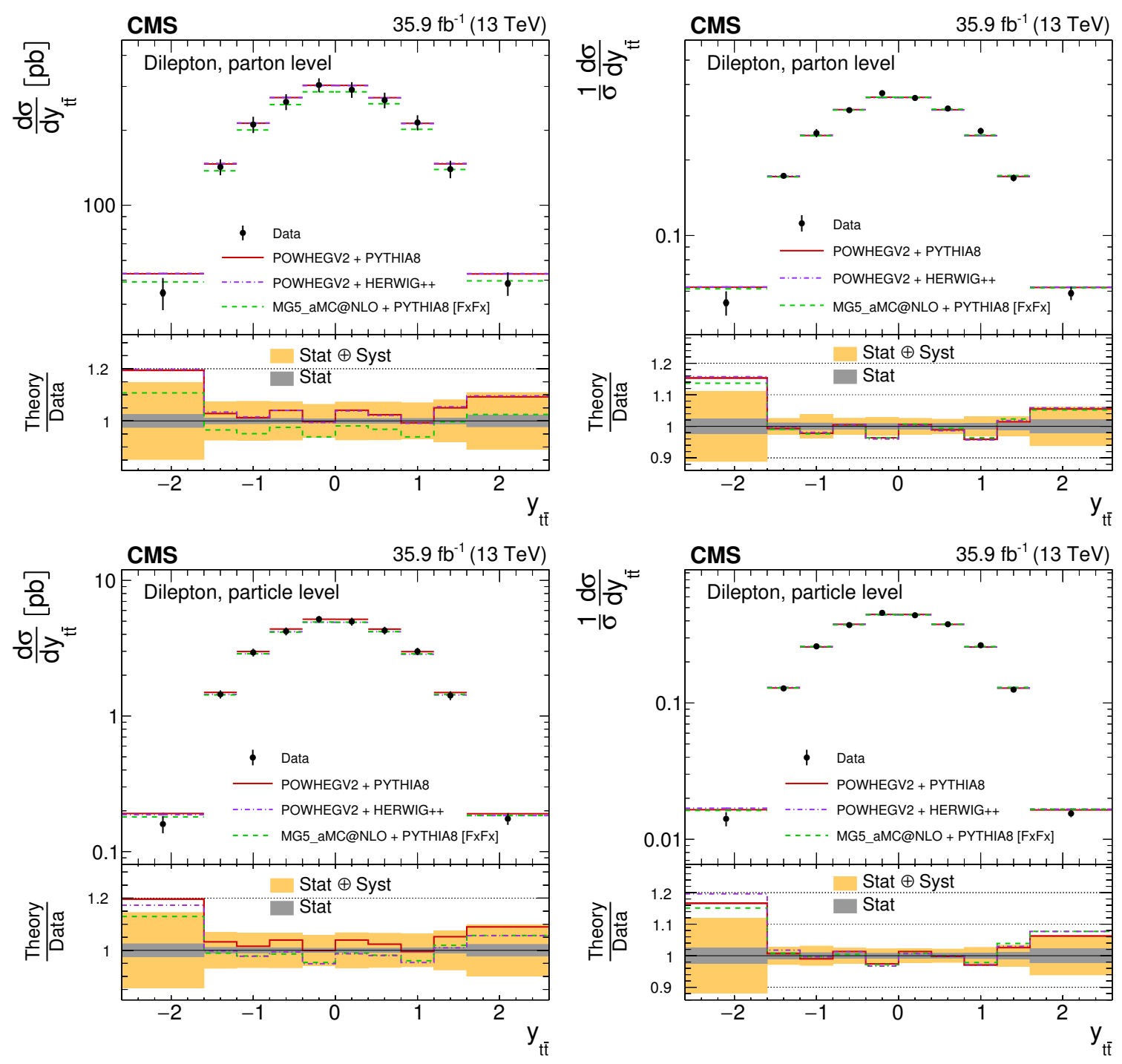

Figure 27. The differential t $\overline{\mathrm{t}}$ production cross sections as a function of $y_{\mathrm{t} \overline{\mathrm{t}}}$ are shown for the data (points) and the MC predictions (lines). The vertical lines on the points indicate the total uncertainty in the data. The left and right columns correspond to absolute and normalised measurements, respectively. The upper row corresponds to measurements at the parton level in the full phase space and the lower row to the particle level in a fiducial phase space. The lower panel in each plot shows the ratios of the theoretical predictions to the data. The dark and light bands show the relative statistical and total uncertainties in the data, respectively. 

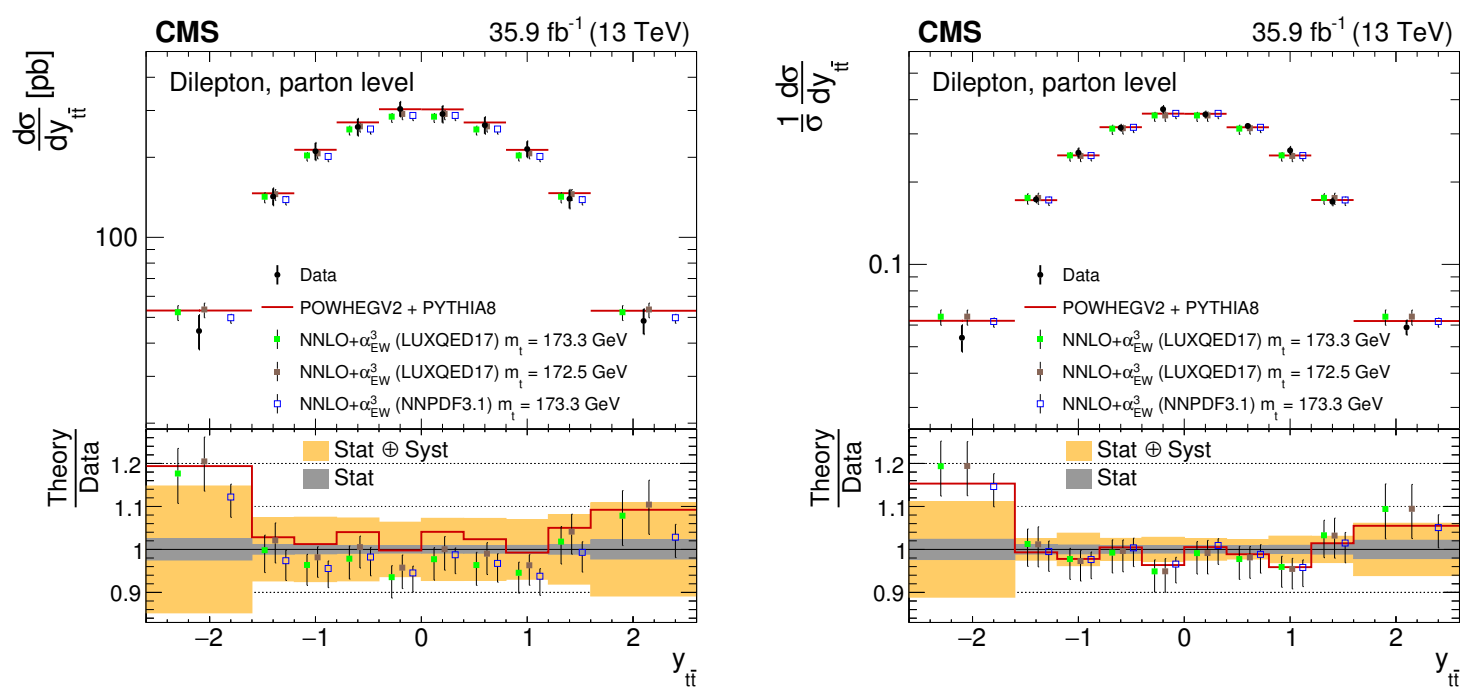

Figure 28. The differential te production cross sections at the parton level in the full phase space as a function of $y_{\mathrm{t} \overline{\mathrm{E}}}$ are shown for the data (filled circles), the theoretical predictions with beyondNLO precision (other points) and the prediction from POWHEG+PYTHIA (solid line). The vertical lines on the filled circles and other points indicate the total uncertainty in the data and theoretical predictions, respectively. The left and right plots correspond to absolute and normalised measurements, respectively. The lower panel in each plot shows the ratios of the theoretical predictions to the data. The dark and light bands show the relative statistical and total uncertainties in the data, respectively.

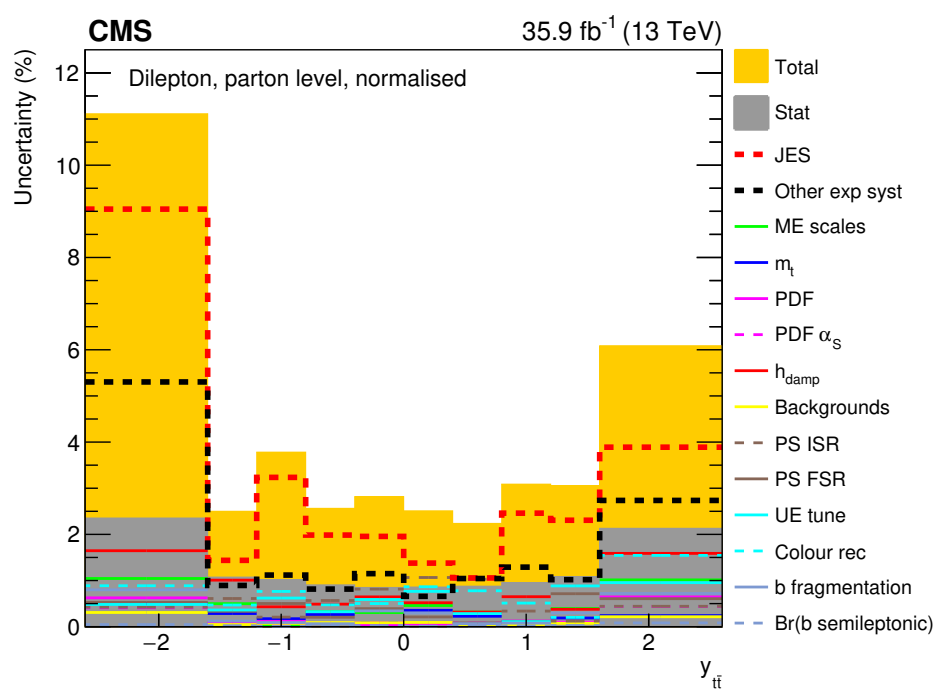

Figure 29. The contributions of each source of systematic uncertainty to the total systematic uncertainty in each bin is shown for the measurement of the normalised t $\bar{t}$ production cross sections as a function of $y_{\mathrm{t} \overline{\mathrm{t}}}$. The sources affecting the JES are added in quadrature and shown as a single component. Additional experimental systematic uncertainties are also added in quadrature and shown as a single component. Contributions from theoretical uncertainties are shown separately. The statistical and total uncertainties, corresponding to the quadrature addition of statistical and systematic uncertainties, are shown by the dark and light filled histograms, respectively. 

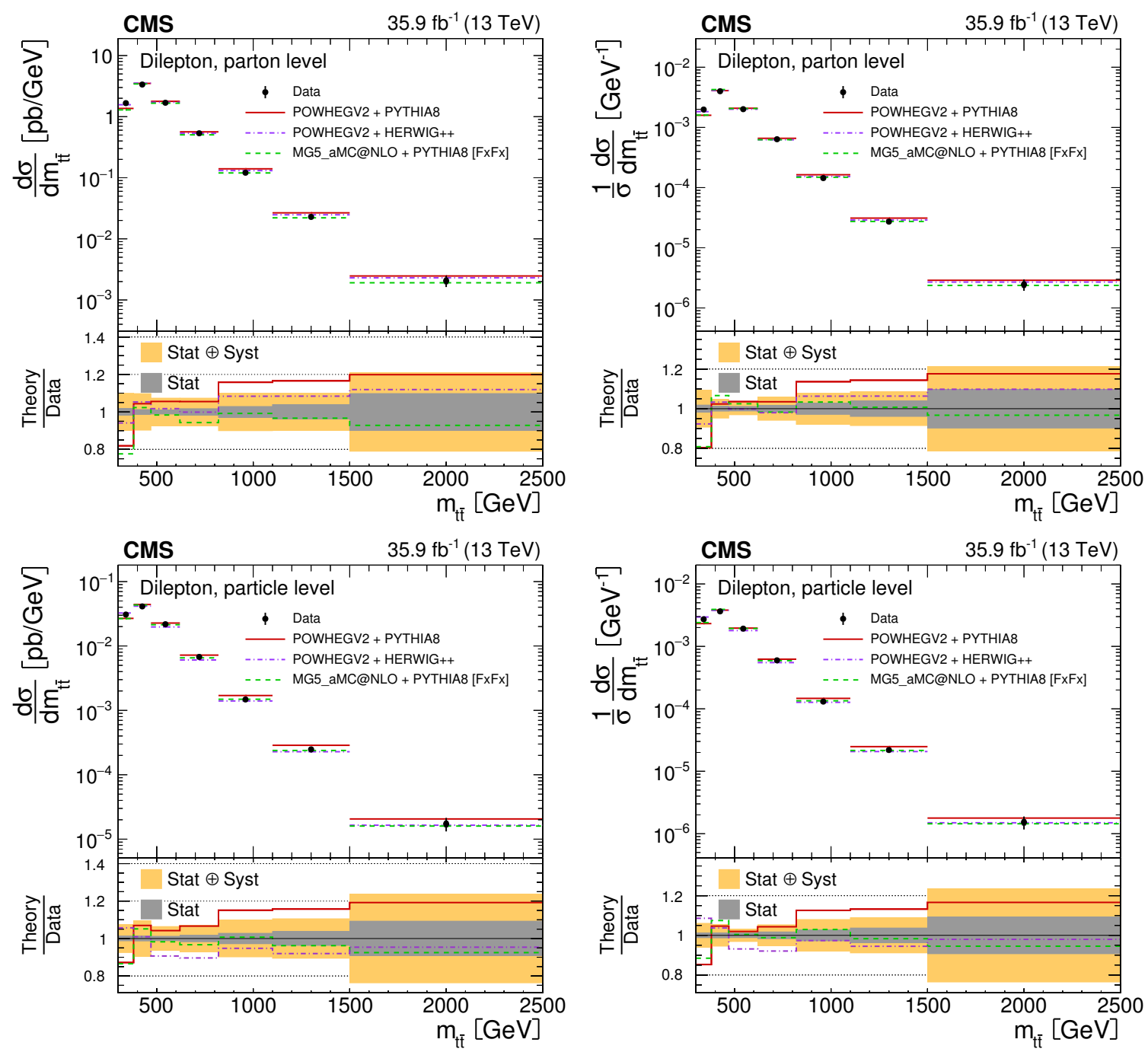

Figure 30. The differential $t \overline{\mathrm{t}}$ production cross sections as a function of $m_{\mathrm{t} \overline{\mathrm{t}}}$ are shown for the data (points) and the MC predictions (lines). The vertical lines on the points indicate the total uncertainty in the data. The left and right columns correspond to absolute and normalised measurements, respectively. The upper row corresponds to measurements at the parton level in the full phase space and the lower row to the particle level in a fiducial phase space. The lower panel in each plot shows the ratios of the theoretical predictions to the data. The dark and light bands show the relative statistical and total uncertainties in the data, respectively. 

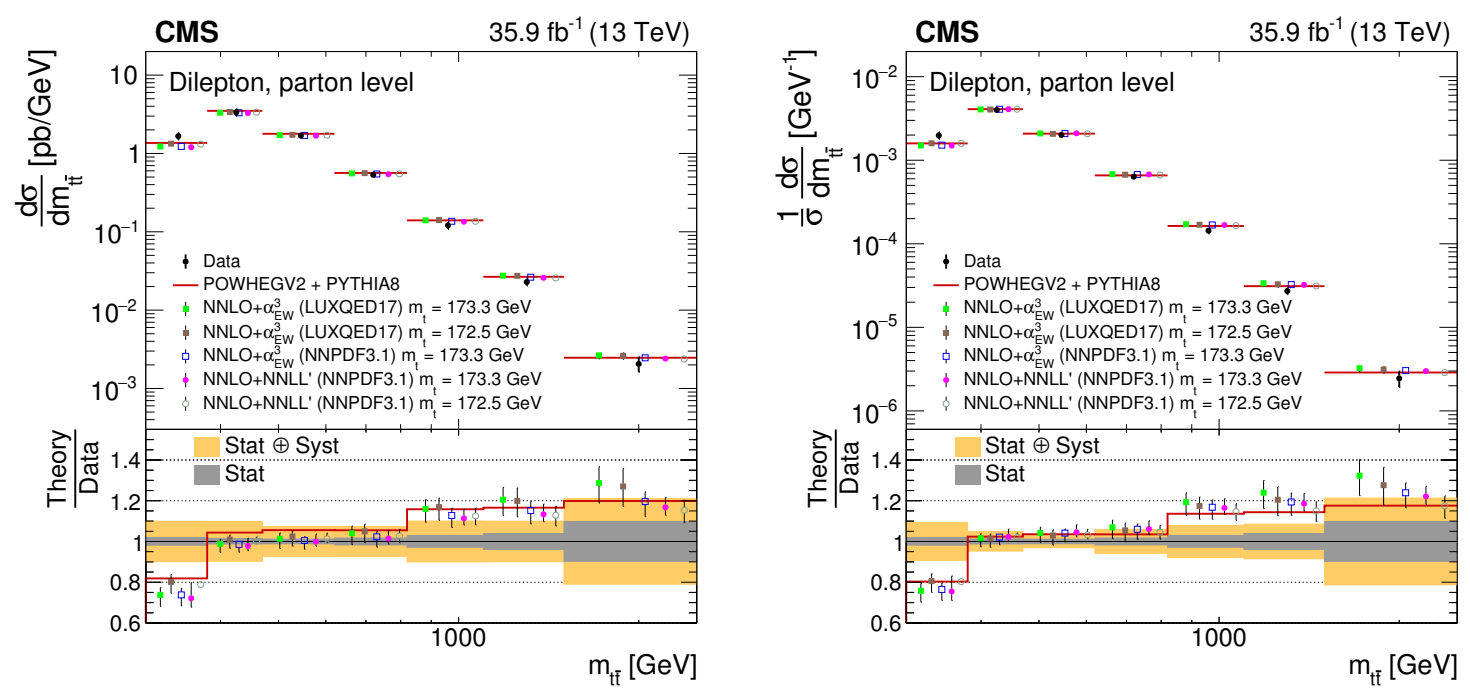

Figure 31. The differential t $\bar{t}$ production cross sections at the parton level in the full phase space as a function of $m_{\mathrm{t} \overline{\mathrm{t}}}$ are shown for the data (filled circles), the theoretical predictions with beyondNLO precision (other points) and the prediction from POWHEG+PYTHIA (solid line). The vertical lines on the filled circles and other points indicate the total uncertainty in the data and theoretical predictions, respectively. The left and right plots correspond to absolute and normalised measurements, respectively. The lower panel in each plot shows the ratios of the theoretical predictions to the data. The dark and light bands show the relative statistical and total uncertainties in the data, respectively.

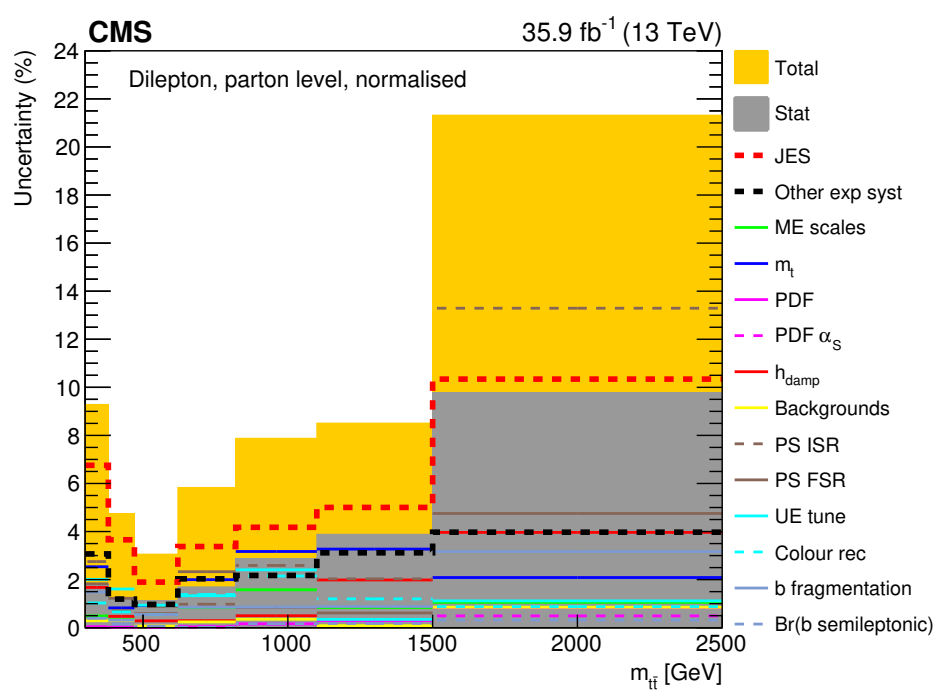

Figure 32. The contributions of each source of systematic uncertainty to the total systematic uncertainty in each bin is shown for the measurement of the normalised $t \bar{t}$ production cross sections as a function of $m_{\mathrm{t} \overline{\mathrm{t}}}$. The sources affecting the JES are added in quadrature and shown as a single component. Additional experimental systematic uncertainties are also added in quadrature and shown as a single component. Contributions from theoretical uncertainties are shown separately. The statistical and total uncertainties, corresponding to the quadrature addition of statistical and systematic uncertainties, are shown by the dark and light filled histograms, respectively. 
CMDM of the top quark, as described in section 8. The measurement of $N_{\text {jets }}$ probes higherorder corrections in the ME calculations and the modelling of radiation in the parton-shower simulations. The $N_{\text {jets }}$ measurement includes the integrated cross section for $N_{\text {jets }}>7$ in the last bin. For all other observables, the first and last bins include the differential cross section integrated within the bin boundaries only.

In tables 48-55 in appendix C, the $\chi^{2}$ per degree of freedom (dof) and corresponding $p$-value are shown, quantifying the agreement between the unfolded data and predictions for all the observables. In addition, figures 52 and 53 summarise the $p$-values for each normalised distribution. For most of the measured observables, we find generally good agreement between data and predictions, within the uncertainties in the data. The cases where significant disagreement is observed are now discussed.

Many of the different top quark $p_{\mathrm{T}}$ distributions shown in figures 3-11 exhibit significant disagreements between the data and the POWHEG+PYTHIA predictions, varying smoothly from an excess of data for low $p_{\mathrm{T}}$ to a deficit for high $p_{\mathrm{T}}$. Comparison of the data to the MG5_amC@NLO+PYTHIA [FXFx] prediction shows a similar excess of data at low $p_{\mathrm{T}}$ but a smaller deficit at high $p_{\mathrm{T}}$. The POWHEG+HERWIG ++ simulation provides a better modelling of the top quark $p_{\mathrm{T}}$ distributions, where a deficit of data for high $p_{\mathrm{T}}$ at the parton level is the only observed disagreement. For all MC-based predictions, the deficit at high $p_{\mathrm{T}}$ is most pronounced for the $p_{\mathrm{T}}^{\mathrm{t}}$ (trailing) distribution. Similar patterns of disagreement were observed at $\sqrt{s}=7,8$, and $13 \mathrm{TeV}$ by the ATLAS [5] and CMS $[8,16,17]$ collaborations. The normalised and absolute $p_{\mathrm{T}}^{\mathrm{t}}$ and $p_{\mathrm{T}}^{\overline{\mathrm{t}}}$ distributions show a similar level of disagreement with the beyond-NLO predictions.

In figure 24, a significant deficit of data with respect to both the POWHEG+PYTHIA and MG5_aMC@NLO+PYTHIA $[F x F x]$ predictions is observed for large values of $p_{\mathrm{T}}^{\mathrm{t} \overline{\mathrm{t}}}$. Conversely, for the POWHEG+HERWIG ++ prediction this deficit is not seen but there is an excess of data at moderate $p_{\mathrm{T}}^{\mathrm{t} \overline{\mathrm{t}}}$. For the NNLO $+\alpha_{\mathrm{EW}}^{3}$ predictions shown in figure 25, a slight deficit of data at high $p_{\mathrm{T}}^{\mathrm{t} \overline{\mathrm{t}}}$ is apparent. For the $m_{\mathrm{t} \overline{\mathrm{t}}}$ distributions in figures 30 and 31, a significant excess of data with respect to all predictions in the lowest bin is observed. This excess is smaller for predictions with $m_{\mathrm{t}}=172.5 \mathrm{GeV}$, which suggests a lower value of $m_{\mathrm{t}}$ could result in improved agreement for this distribution.

The distributions of kinematic properties of the leptons, $\mathrm{b}$ jets, dileptons, and $\mathrm{b}$ jet pairs $\left(p_{\mathrm{T}}^{\ell}, p_{\mathrm{T}}^{\mathrm{b}}, p_{\mathrm{T}}^{\ell \bar{\ell}}, p_{\mathrm{T}}^{\mathrm{b} \overline{\mathrm{b}}}, m_{\ell \bar{\ell}}\right.$, and $\left.m_{\mathrm{b} \overline{\mathrm{b}}}\right)$ in figures $33-50$ exhibit similar disagreements with the predictions as the corresponding top-quark-based observables $p_{\mathrm{T}}^{\mathrm{t}}$ and $p_{\mathrm{T}}^{\mathrm{t} \overline{\mathrm{t}}}$ with which they are correlated. In figure 51, an increasing excess of data over the POWHEG+PYTHIA and POWHEG + HERWIG ++ predictions is observed for $N_{\text {jets }} \geq 4$. Conversely, there is good agreement between MG5_amC@NLO+PYTHIA [FxFx] and data for $N_{\text {jets }}>3$, but disagreement for $N_{\text {jets }}=2,3$.

\section{Constraining the top quark CMDM}

In the SM, the intrinsic spin and colour charge of the top quark give it a small magnetic dipole moment in the colour fields known as the top quark CMDM. An anomalous top quark CMDM is a feature of several BSM scenarios and can affect both the rate and kinematic 

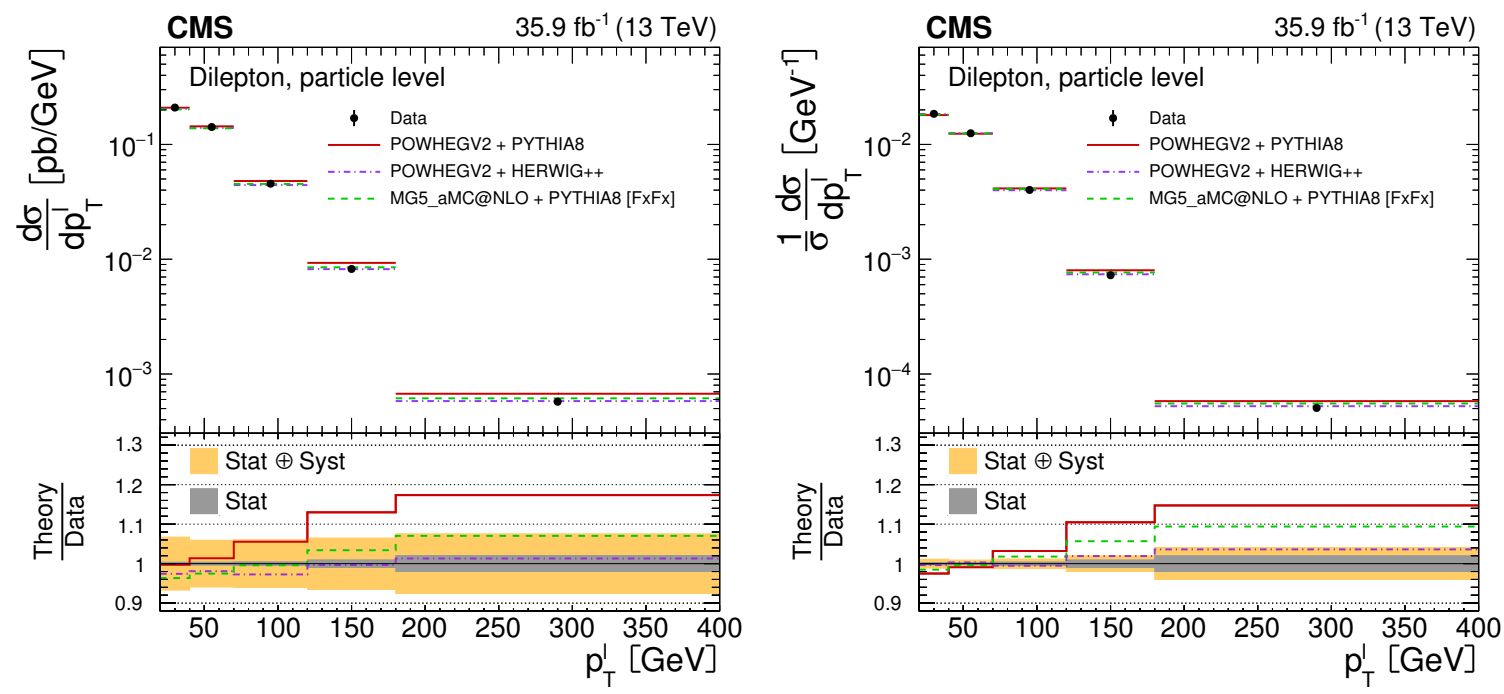

Figure 33. The differential t $\bar{t}$ production cross sections as a function of $p_{\mathrm{T}}^{\ell}$ in a fiducial phase space at the particle level are shown for the data (points) and the MC predictions (lines). The vertical lines on the points indicate the total uncertainty in the data. The left and right plots correspond to absolute and normalised measurements, respectively. The lower panel in each plot shows the ratios of the theoretical predictions to the data. The dark and light bands show the relative statistical and total uncertainties in the data, respectively.
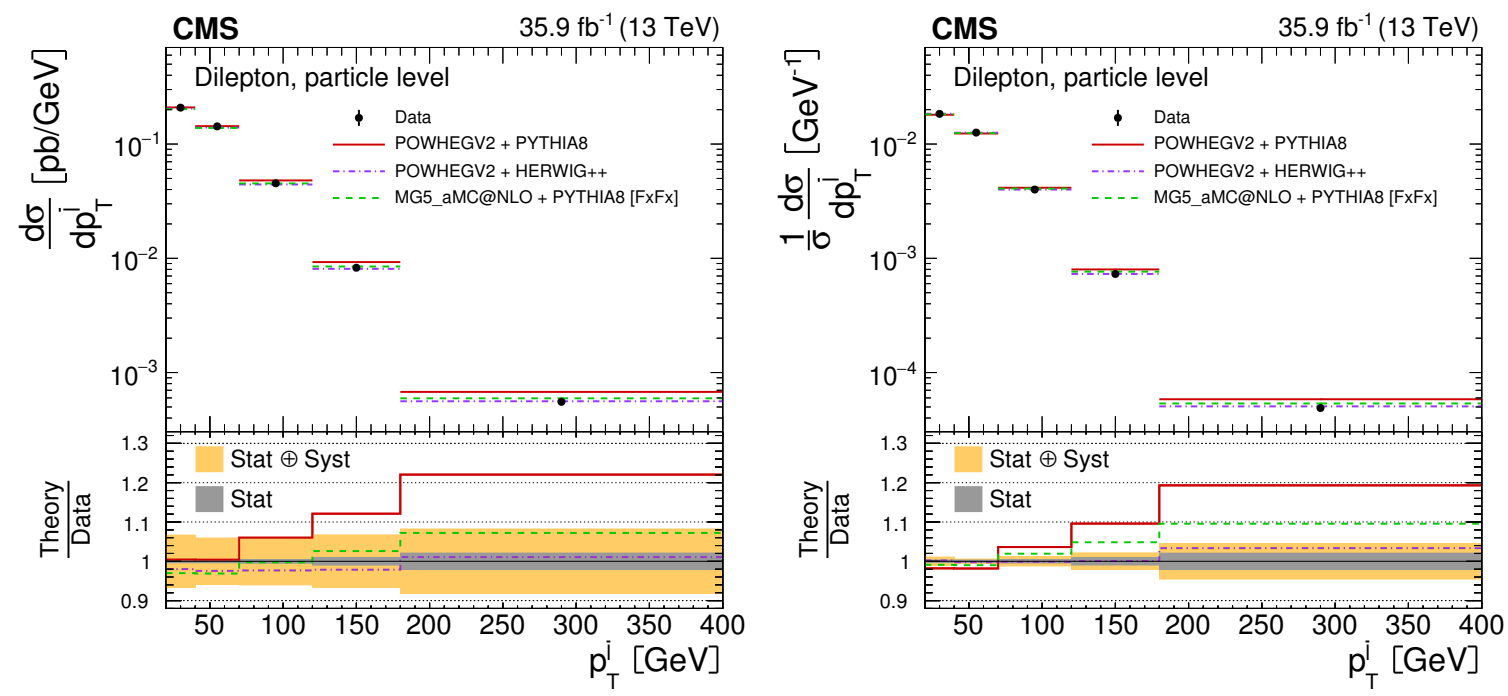

Figure 34. The differential t $\bar{t}$ production cross sections as a function of $p_{\mathrm{T}}^{\bar{\ell}}$ in a fiducial phase space at the particle level are shown for the data (points) and the MC predictions (lines). The vertical lines on the points indicate the total uncertainty in the data. The left and right plots correspond to absolute and normalised measurements, respectively. The lower panel in each plot shows the ratios of the theoretical predictions to the data. The dark and light bands show the relative statistical and total uncertainties in the data, respectively. 

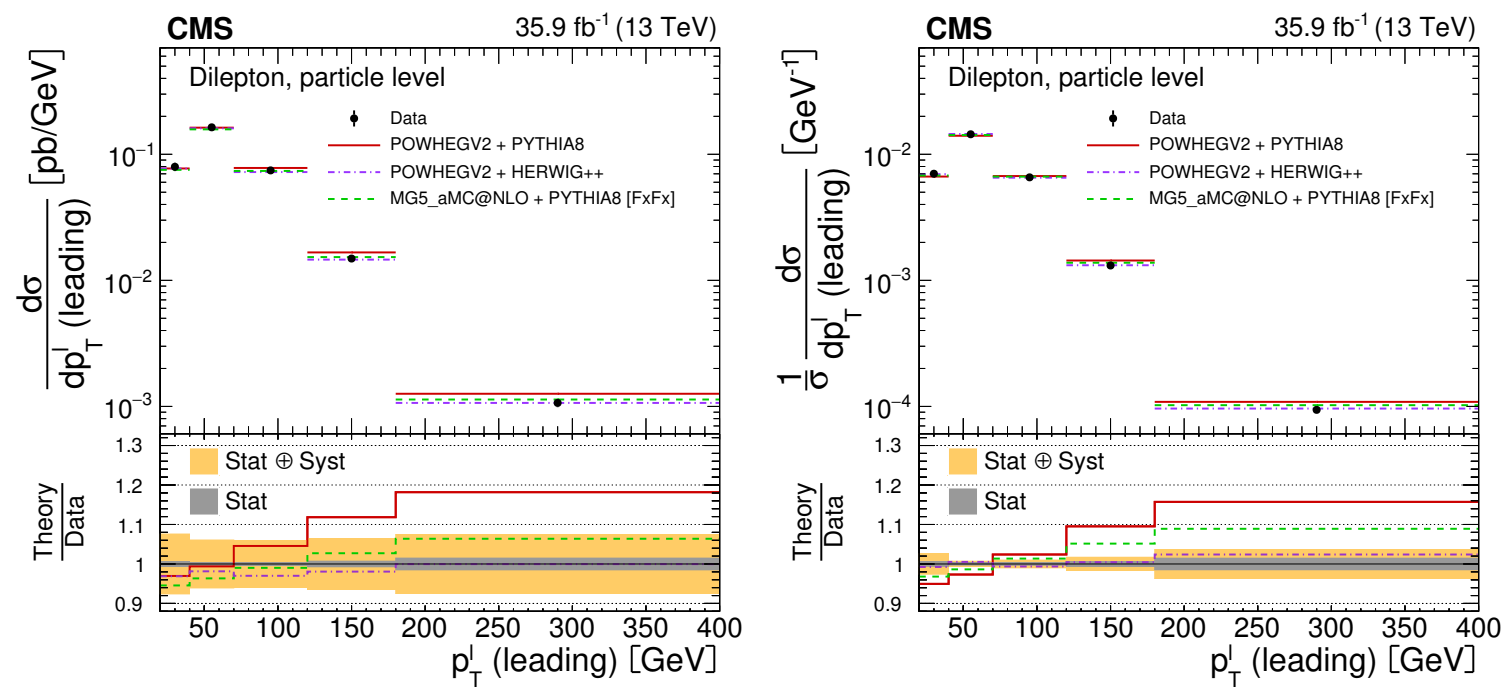

Figure 35. The differential t $\overline{\mathrm{t}}$ production cross sections as a function of $p_{\mathrm{T}}^{\ell}$ (leading) in a fiducial phase space at the particle level are shown for the data (points) and the MC predictions (lines). The vertical lines on the points indicate the total uncertainty in the data. The left and right plots correspond to absolute and normalised measurements, respectively. The lower panel in each plot shows the ratios of the theoretical predictions to the data. The dark and light bands show the relative statistical and total uncertainties in the data, respectively.
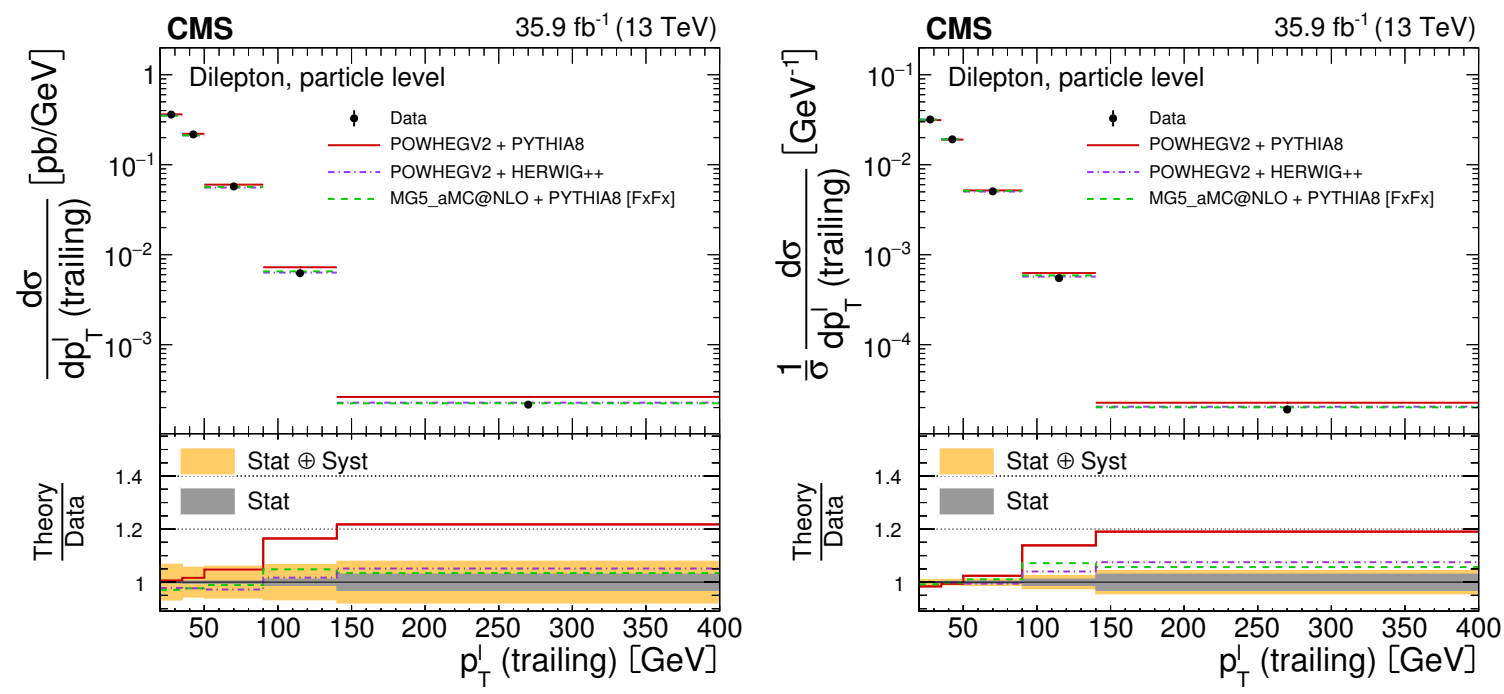

Figure 36. The differential t $\bar{t}$ production cross sections as a function of $p_{\mathrm{T}}^{\ell}$ (trailing) in a fiducial phase space at the particle level are shown for the data (points) and the MC predictions (lines). The vertical lines on the points indicate the total uncertainty in the data. The left and right plots correspond to absolute and normalised measurements, respectively. The lower panel in each plot shows the ratios of the theoretical predictions to the data. The dark and light bands show the relative statistical and total uncertainties in the data, respectively. 

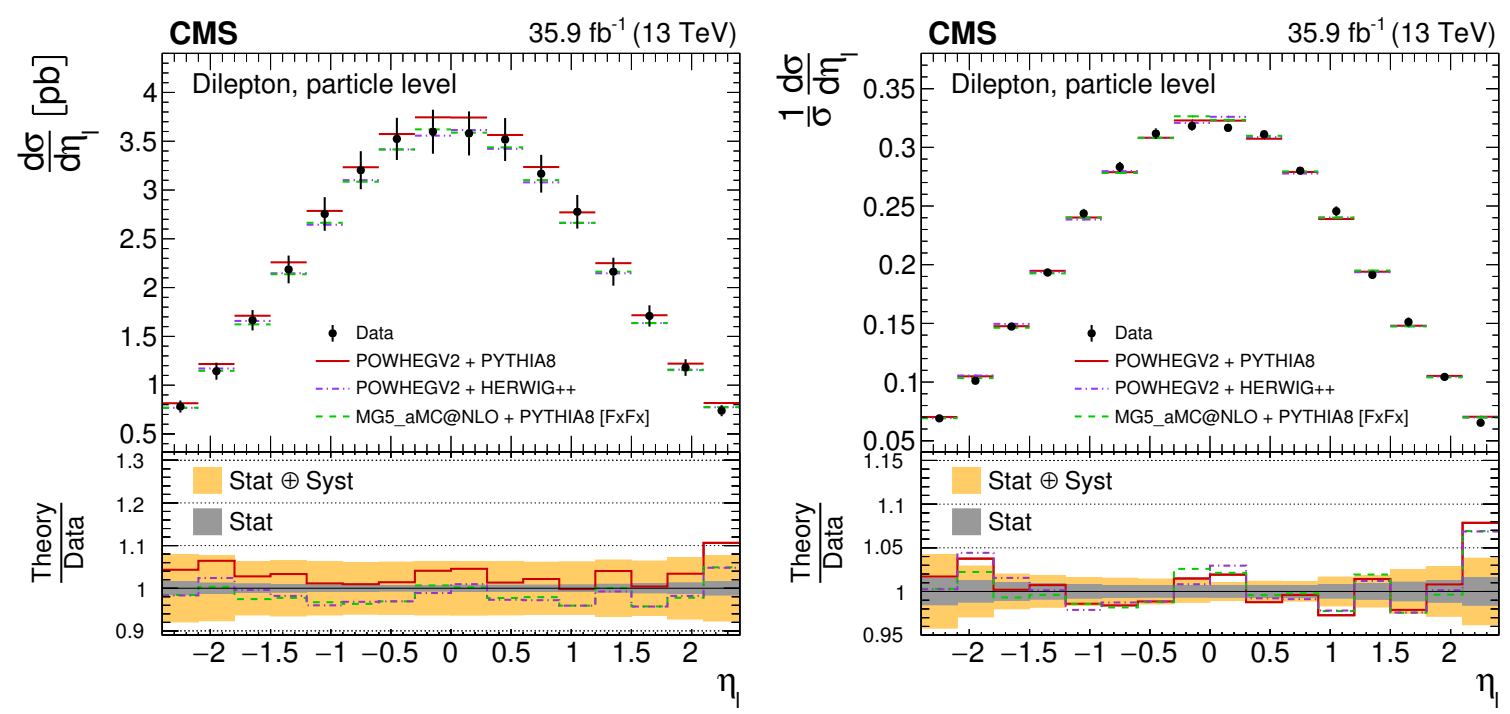

Figure 37. The differential t $\bar{t}$ production cross sections as a function of $\eta_{\ell}$ in a fiducial phase space at the particle level are shown for the data (points) and the MC predictions (lines). The vertical lines on the points indicate the total uncertainty in the data. The left and right plots correspond to absolute and normalised measurements, respectively. The lower panel in each plot shows the ratios of the theoretical predictions to the data. The dark and light bands show the relative statistical and total uncertainties in the data, respectively.
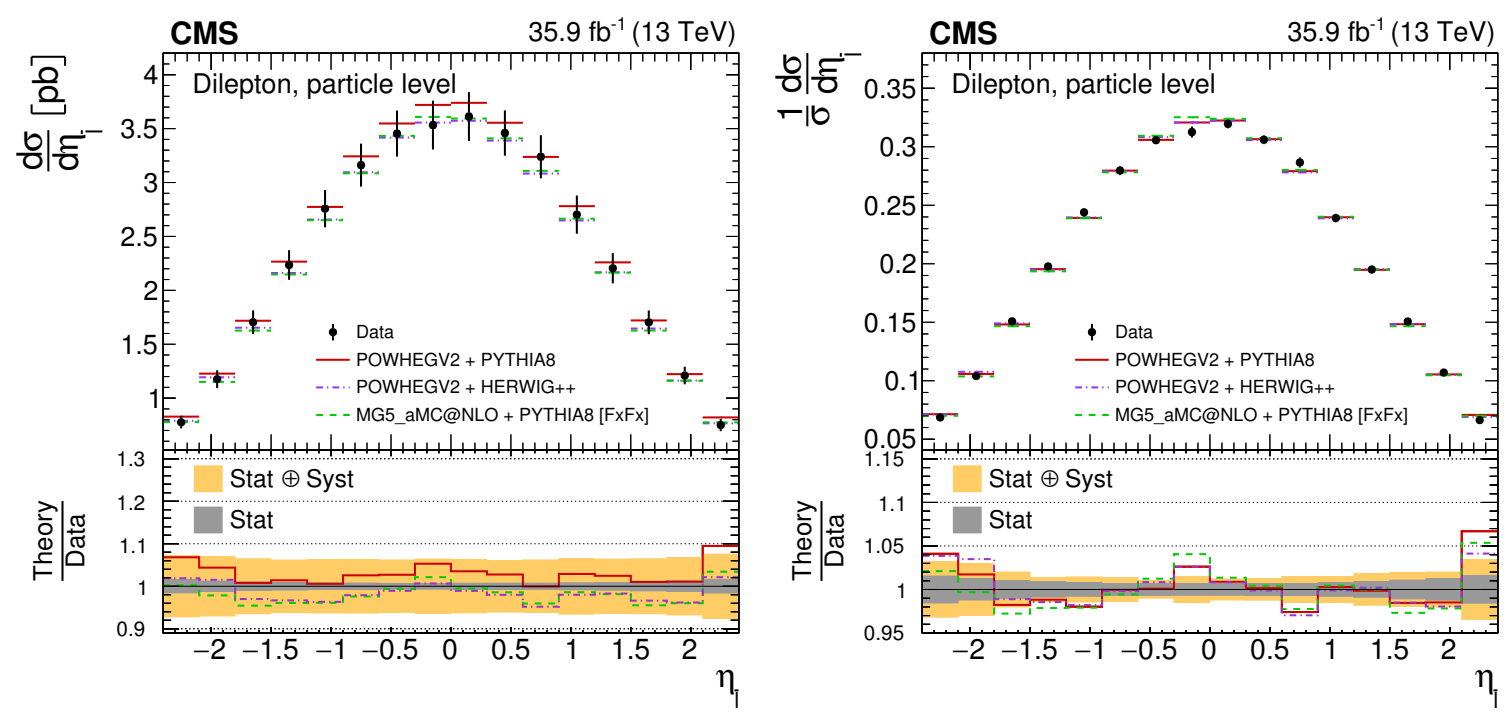

Figure 38. The differential t $\bar{t}$ production cross sections as a function of $\eta_{\bar{\ell}}$ in a fiducial phase space at the particle level are shown for the data (points) and the MC predictions (lines). The vertical lines on the points indicate the total uncertainty in the data. The left and right plots correspond to absolute and normalised measurements, respectively. The lower panel in each plot shows the ratios of the theoretical predictions to the data. The dark and light bands show the relative statistical and total uncertainties in the data, respectively. 

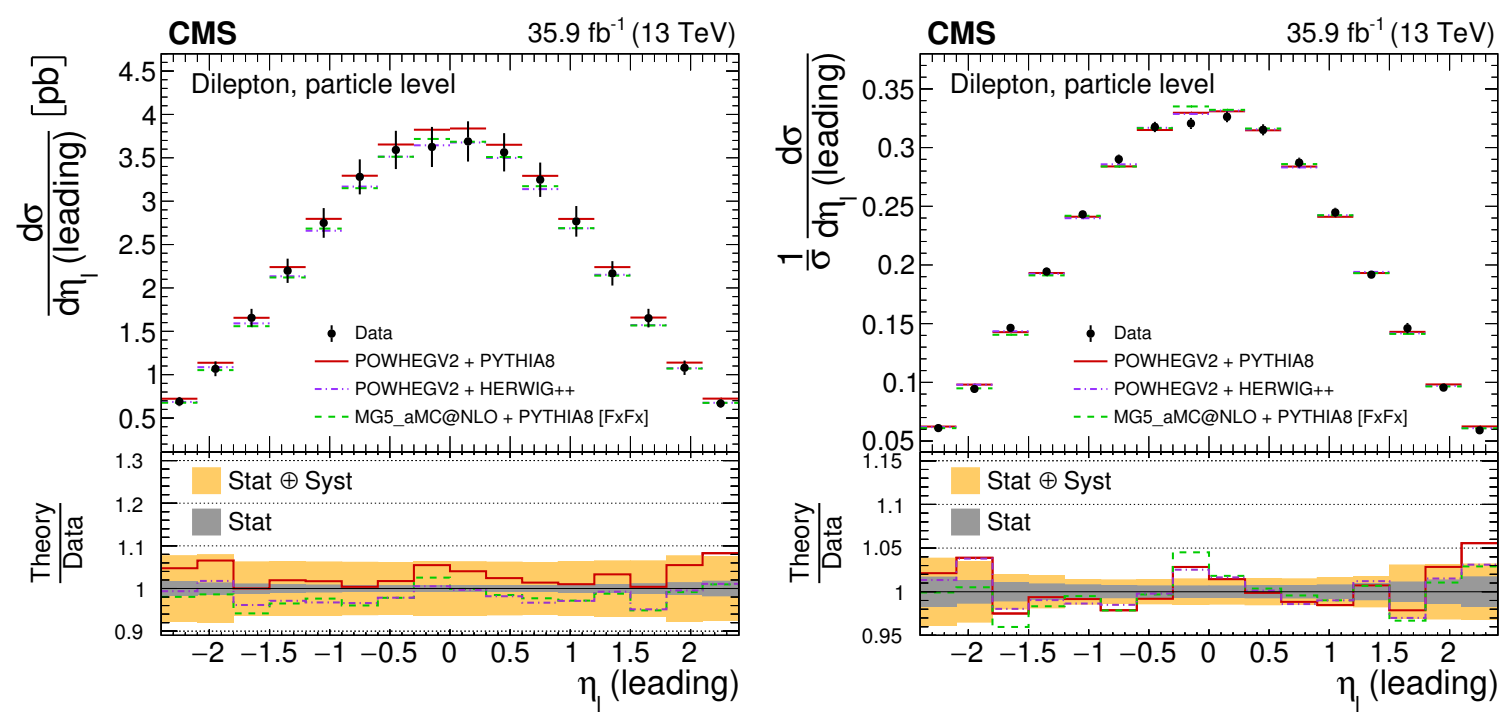

Figure 39. The differential t $\bar{t}$ production cross sections as a function of $\eta_{\ell}$ (leading) in a fiducial phase space at the particle level are shown for the data (points) and the MC predictions (lines). The vertical lines on the points indicate the total uncertainty in the data. The left and right plots correspond to absolute and normalised measurements, respectively. The lower panel in each plot shows the ratios of the theoretical predictions to the data. The dark and light bands show the relative statistical and total uncertainties in the data, respectively.
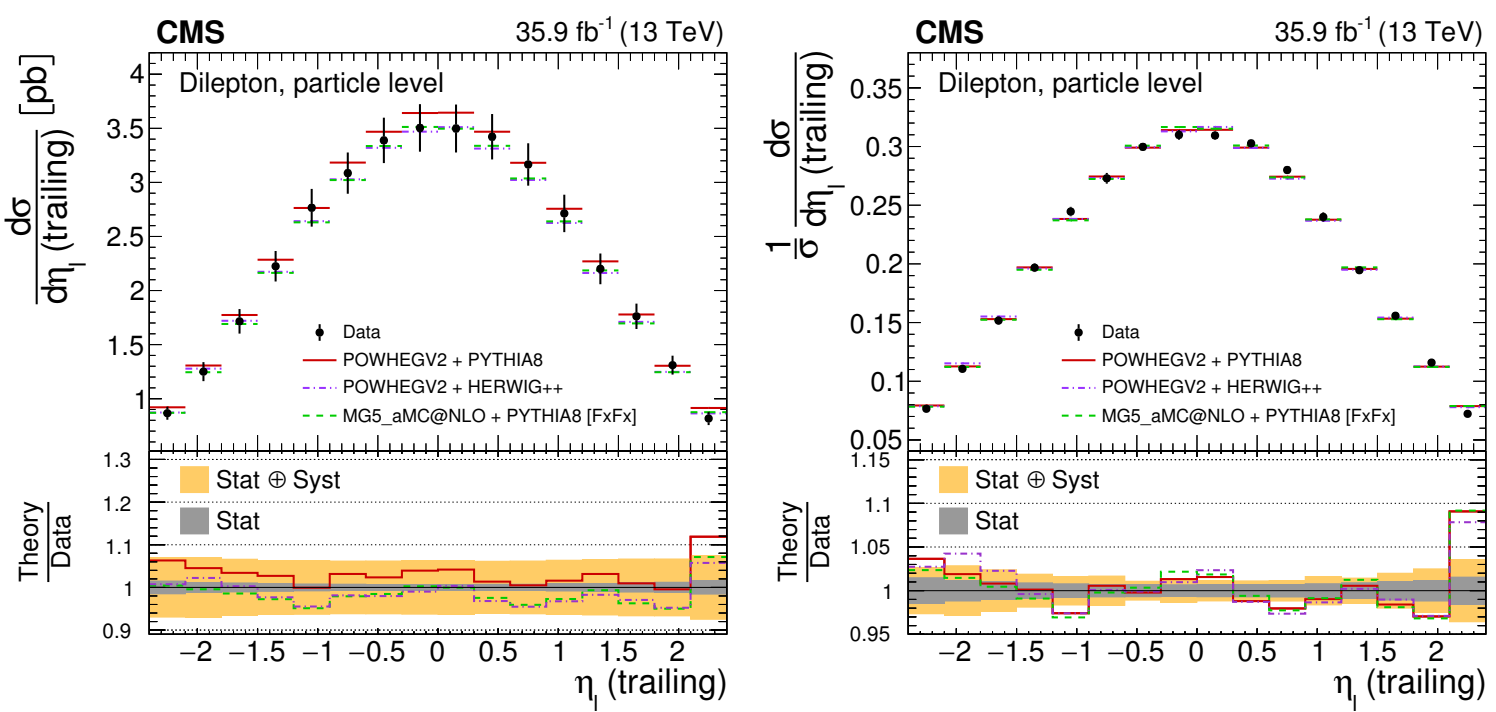

Figure 40. The differential t $\overline{\mathrm{t}}$ production cross sections as a function of $\eta_{\ell}$ (trailing) in a fiducial phase space at the particle level are shown for the data (points) and the MC predictions (lines). The vertical lines on the points indicate the total uncertainty in the data. The left and right plots correspond to absolute and normalised measurements, respectively. The lower panel in each plot shows the ratios of the theoretical predictions to the data. The dark and light bands show the relative statistical and total uncertainties in the data, respectively. 

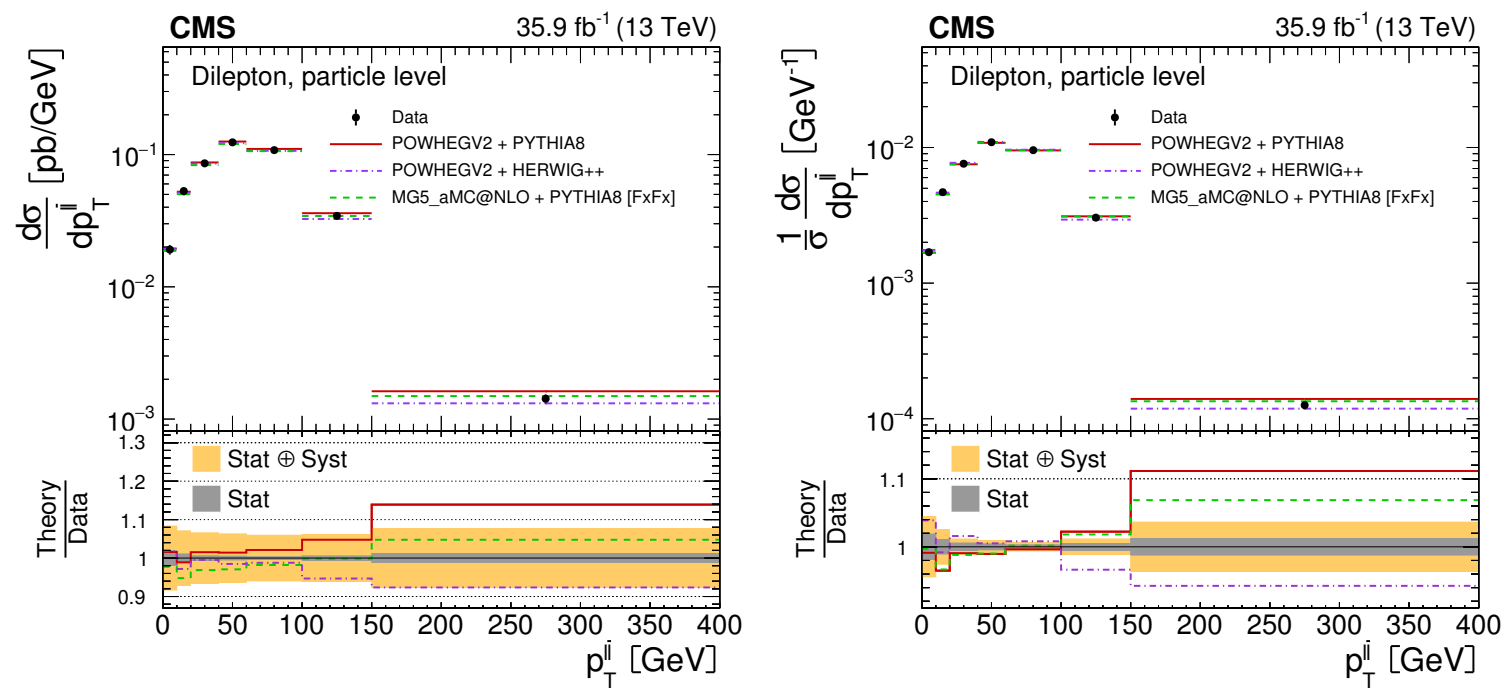

Figure 41. The differential t $\overline{\mathrm{t}}$ production cross sections as a function of $p_{\mathrm{T}}^{\ell \bar{\ell}}$ in a fiducial phase space at the particle level are shown for the data (points) and the MC predictions (lines). The vertical lines on the points indicate the total uncertainty in the data. The left and right plots correspond to absolute and normalised measurements, respectively. The lower panel in each plot shows the ratios of the theoretical predictions to the data. The dark and light bands show the relative statistical and total uncertainties in the data, respectively.
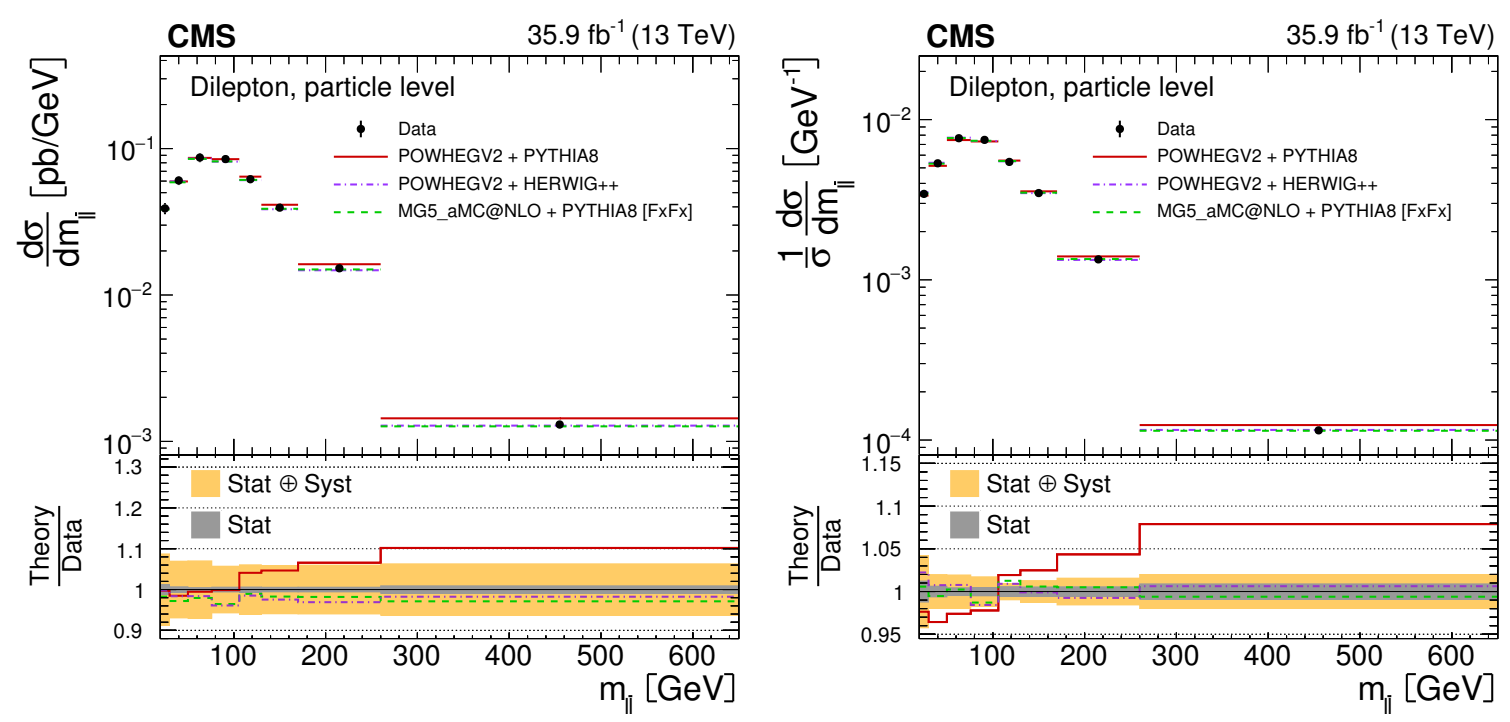

Figure 42. The differential t $\mathrm{t}$ production cross sections as a function of $m_{\ell \bar{\ell}}$ in a fiducial phase space at the particle level are shown for the data (points) and the MC predictions (lines). The vertical lines on the points indicate the total uncertainty in the data. The left and right plots correspond to absolute and normalised measurements, respectively. The lower panel in each plot shows the ratios of the theoretical predictions to the data. The dark and light bands show the relative statistical and total uncertainties in the data, respectively. 

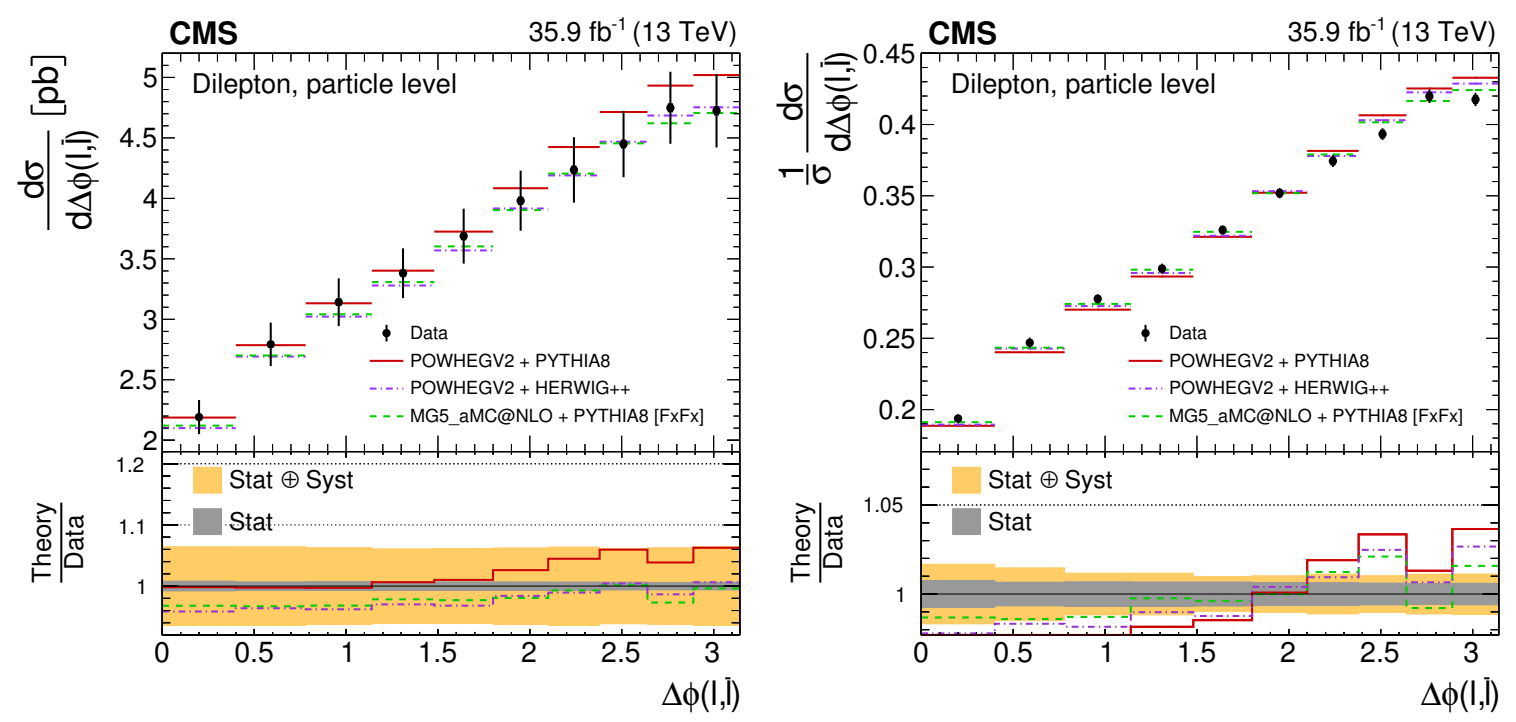

Figure 43. The differential t $\bar{t}$ production cross sections as a function of $\Delta \phi(\ell, \bar{\ell})$ in a fiducial phase space at the particle level are shown for the data (points) and the MC predictions (lines). The vertical lines on the points indicate the total uncertainty in the data. The left and right plots correspond to absolute and normalised measurements, respectively. The lower panel in each plot shows the ratios of the theoretical predictions to the data. The dark and light bands show the relative statistical and total uncertainties in the data, respectively.
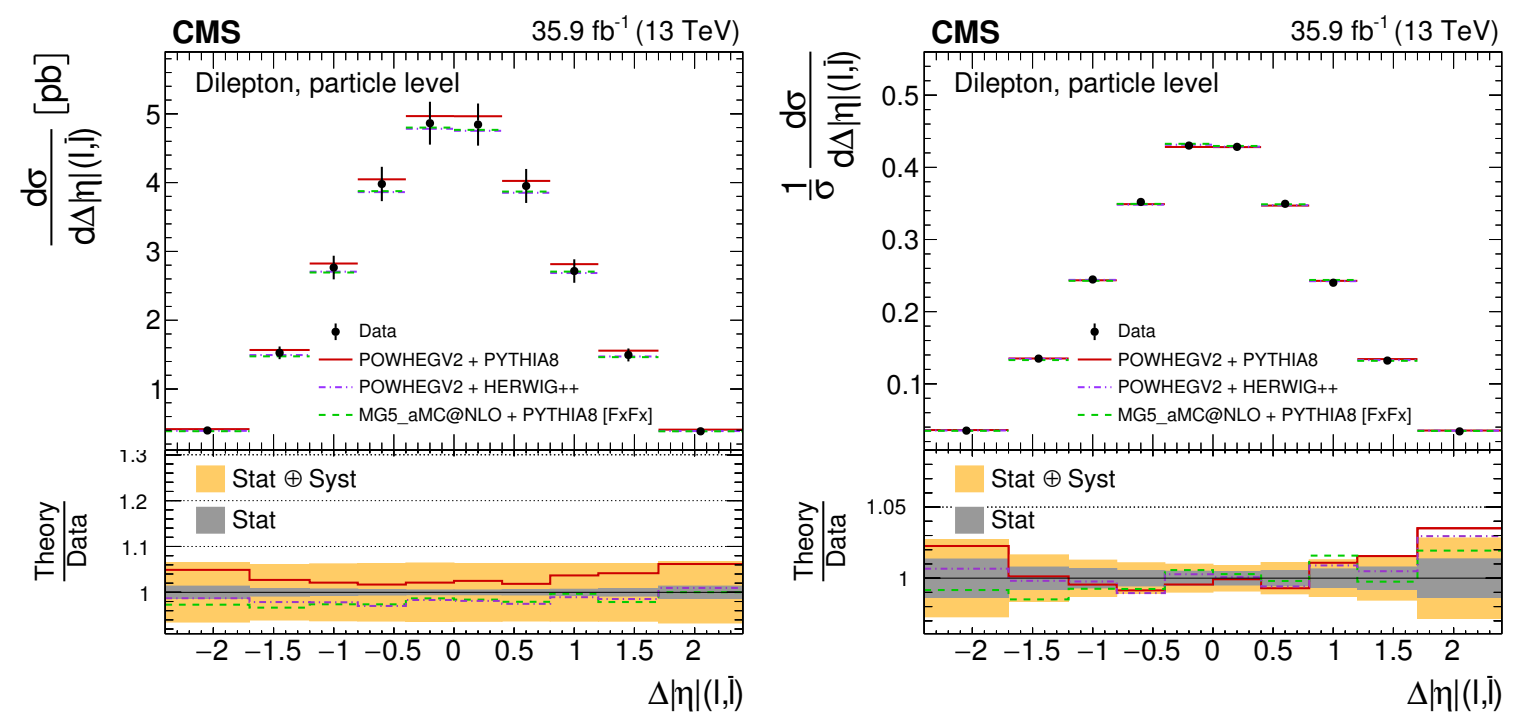

Figure 44. The differential $t \bar{t}$ production cross sections as a function of $\Delta \eta(\ell, \bar{\ell})$ in a fiducial phase space at the particle level are shown for the data (points) and the MC predictions (lines). The vertical lines on the points indicate the total uncertainty in the data. The left and right plots correspond to absolute and normalised measurements, respectively. The lower panel in each plot shows the ratios of the theoretical predictions to the data. The dark and light bands show the relative statistical and total uncertainties in the data, respectively. 

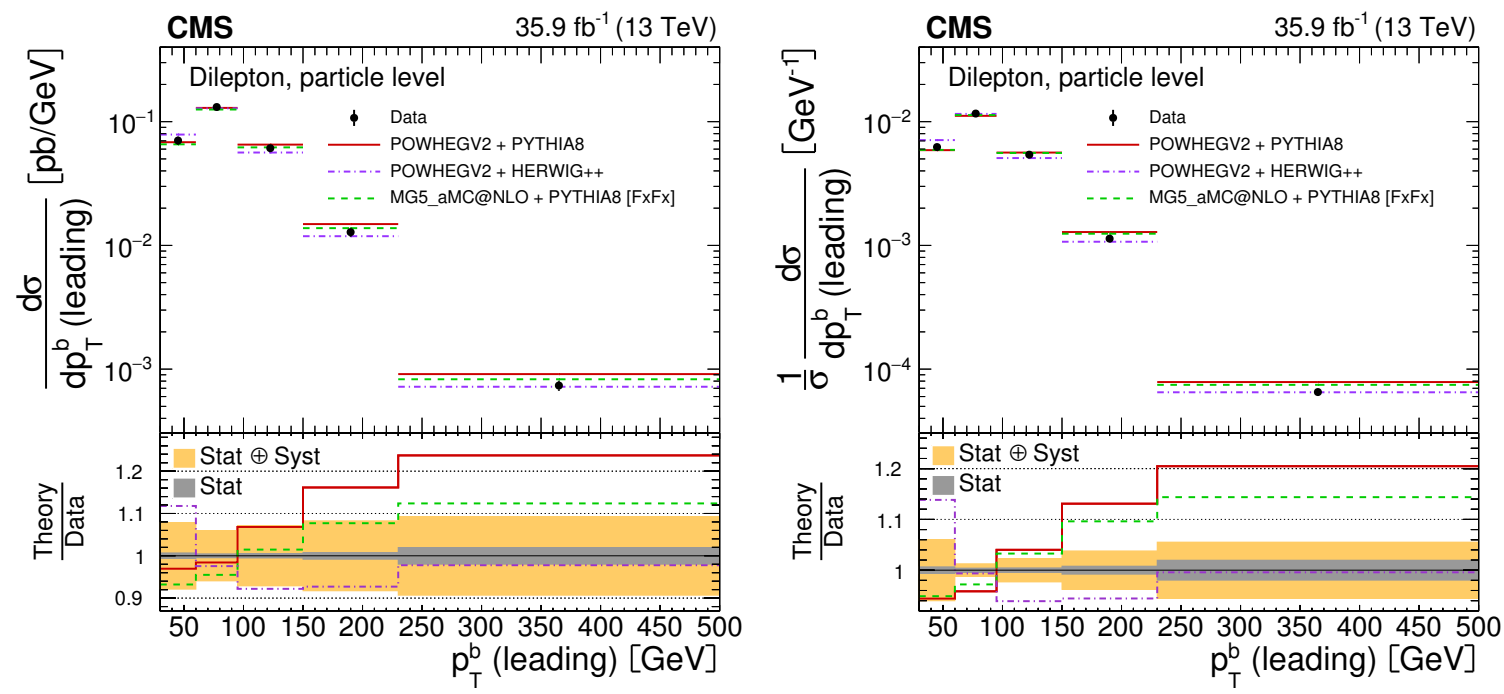

Figure 45. The differential t $\overline{\mathrm{t}}$ production cross sections as a function of $p_{\mathrm{T}}^{\mathrm{b}}$ (leading) in a fiducial phase space at the particle level are shown for the data (points) and the MC predictions (lines). The vertical lines on the points indicate the total uncertainty in the data. The left and right plots correspond to absolute and normalised measurements, respectively. The lower panel in each plot shows the ratios of the theoretical predictions to the data. The dark and light bands show the relative statistical and total uncertainties in the data, respectively.
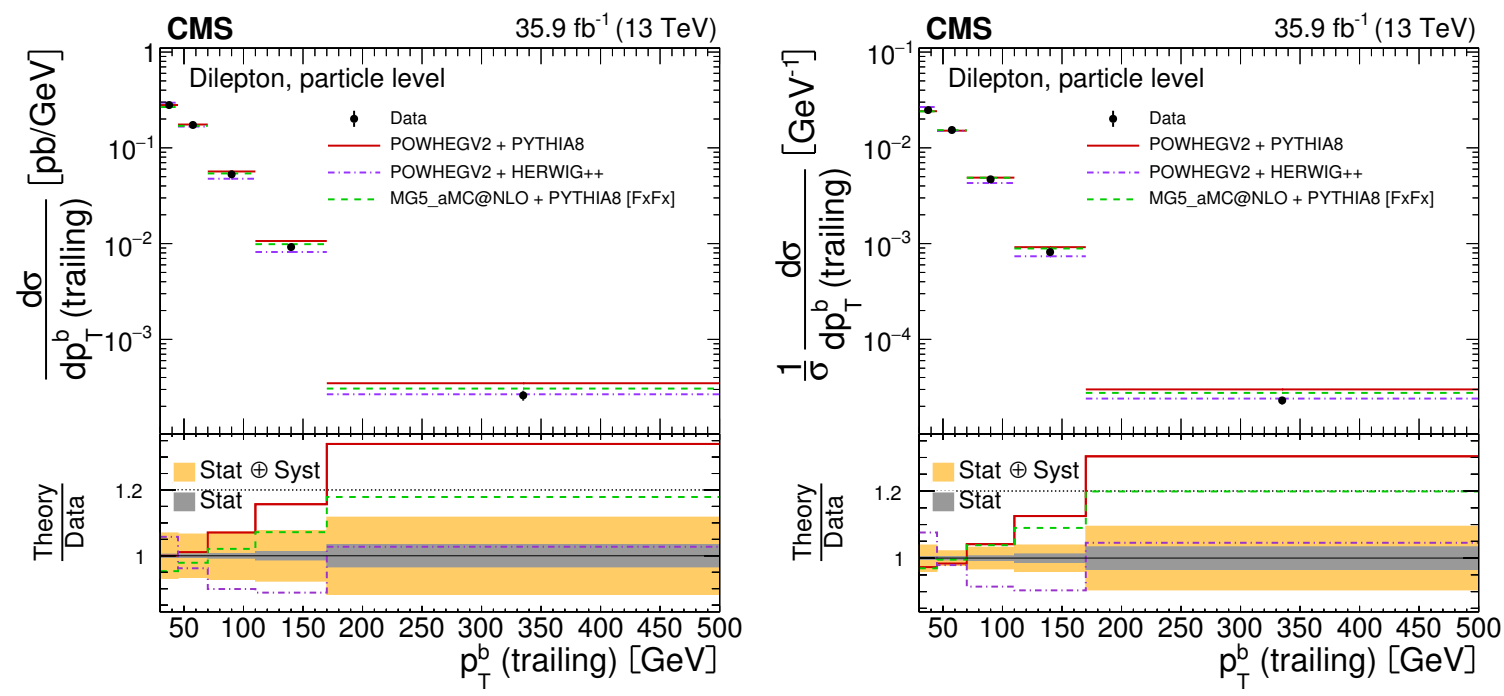

Figure 46. The differential t $\overline{\mathrm{t}}$ production cross sections as a function of $p_{\mathrm{T}}^{\mathrm{b}}$ (trailing) in a fiducial phase space at the particle level are shown for the data (points) and the MC predictions (lines). The vertical lines on the points indicate the total uncertainty in the data. The left and right plots correspond to absolute and normalised measurements, respectively. The lower panel in each plot shows the ratios of the theoretical predictions to the data. The dark and light bands show the relative statistical and total uncertainties in the data, respectively. 

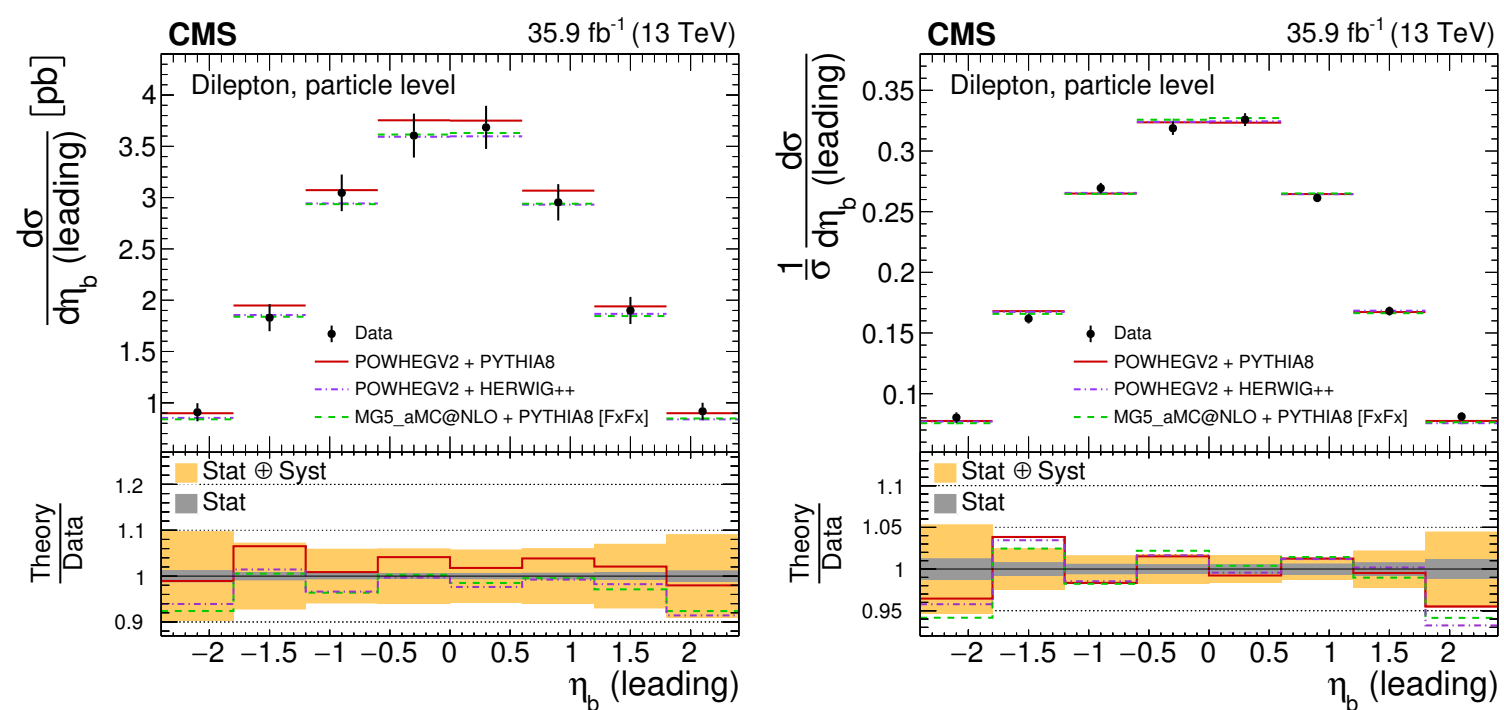

Figure 47. The differential t t $\overline{\mathrm{t}}$ production cross sections as a function of $\eta_{\mathrm{b}}$ (leading) in a fiducial phase space at the particle level are shown for the data (points) and the MC predictions (lines). The vertical lines on the points indicate the total uncertainty in the data. The left and right plots correspond to absolute and normalised measurements, respectively. The lower panel in each plot shows the ratios of the theoretical predictions to the data. The dark and light bands show the relative statistical and total uncertainties in the data, respectively.
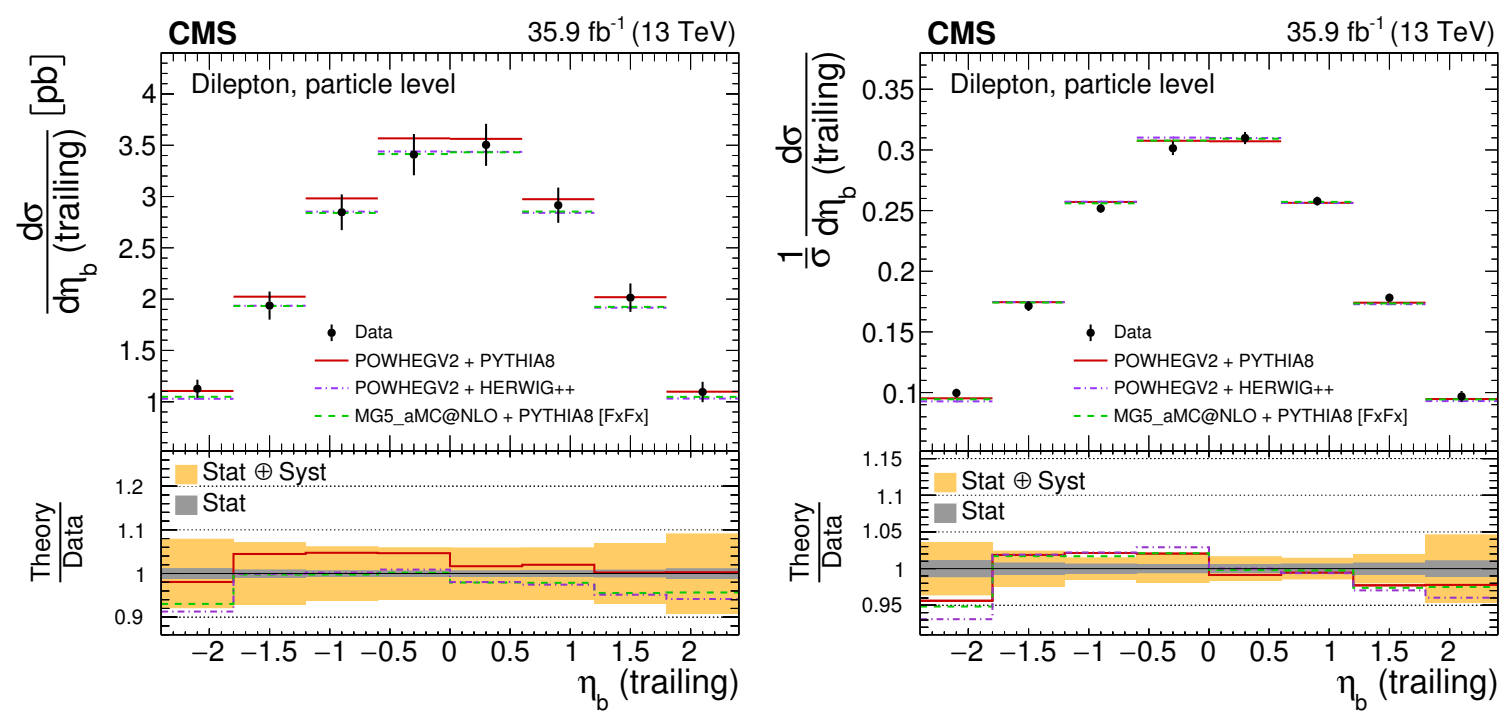

Figure 48. The differential t $\overline{\mathrm{t}}$ production cross sections as a function of $\eta_{\mathrm{b}}$ (trailing) in a fiducial phase space at the particle level are shown for the data (points) and the MC predictions (lines). The vertical lines on the points indicate the total uncertainty in the data. The left and right plots correspond to absolute and normalised measurements, respectively. The lower panel in each plot shows the ratios of the theoretical predictions to the data. The dark and light bands show the relative statistical and total uncertainties in the data, respectively. 

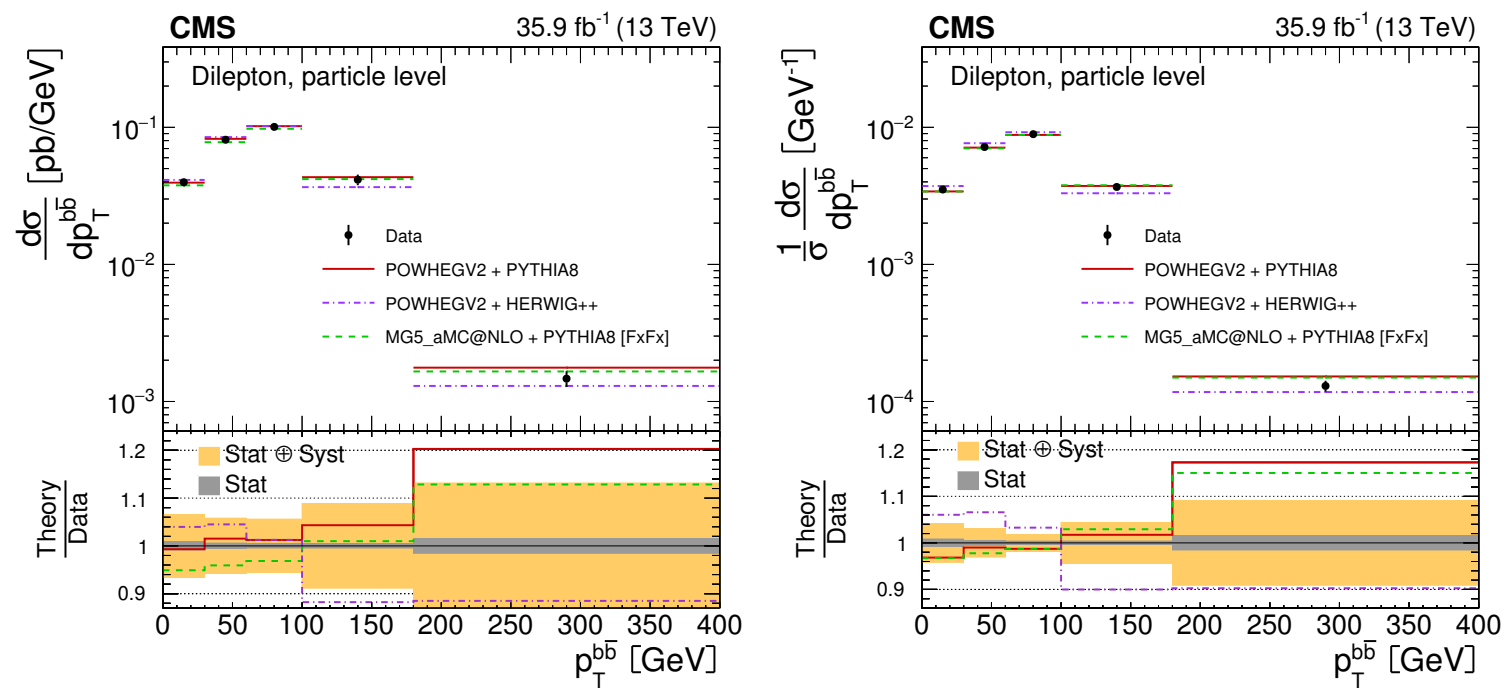

Figure 49. The differential t $\overline{\mathrm{t}}$ production cross sections as a function of $p_{\mathrm{T}}^{\mathrm{b} \overline{\mathrm{b}}}$ in a fiducial phase space at the particle level are shown for the data (points) and the MC predictions (lines). The vertical lines on the points indicate the total uncertainty in the data. The left and right plots correspond to absolute and normalised measurements, respectively. The lower panel in each plot shows the ratios of the theoretical predictions to the data. The dark and light bands show the relative statistical and total uncertainties in the data, respectively.
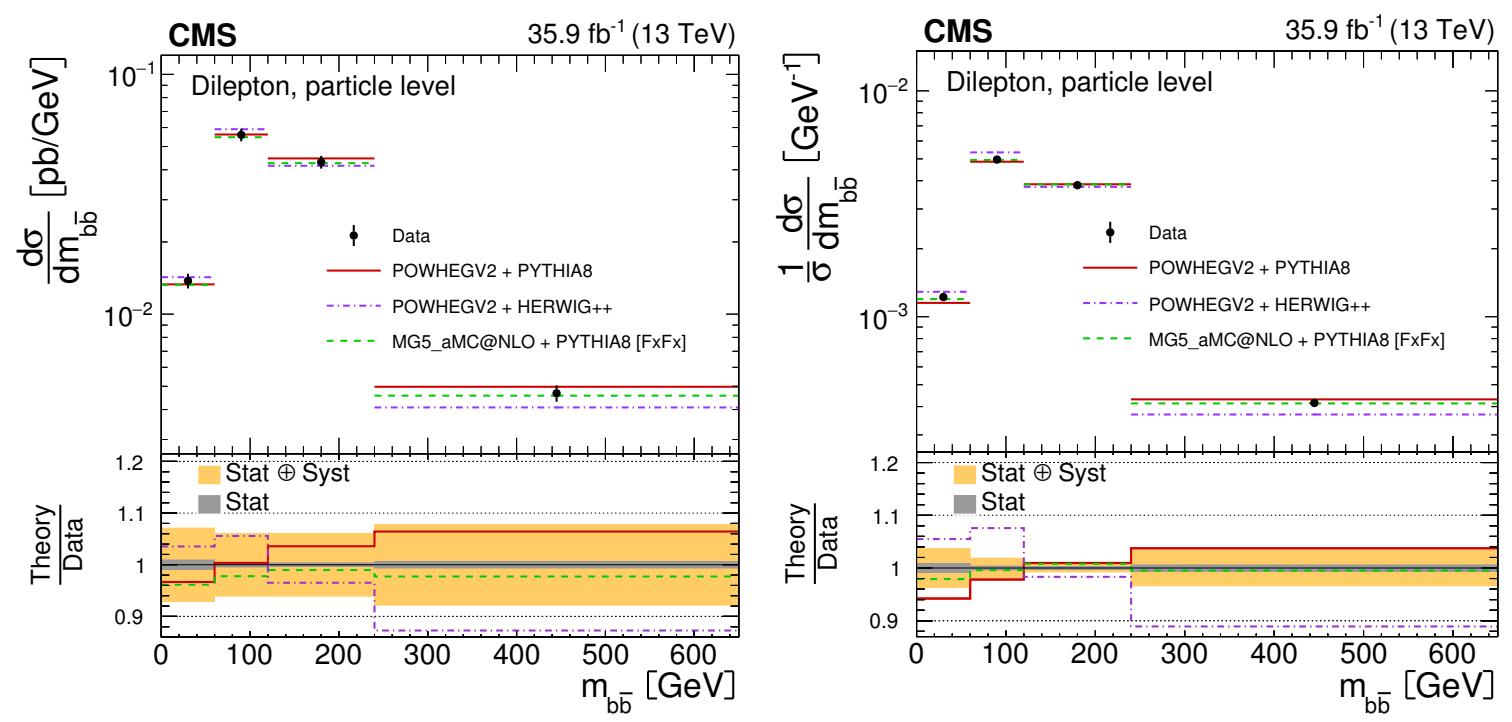

Figure 50. The differential t $\bar{t}$ production cross sections as a function of $m_{\mathrm{b} \overline{\mathrm{b}}}$ in a fiducial phase space at the particle level are shown for the data (points) and the MC predictions (lines). The vertical lines on the points indicate the total uncertainty in the data. The left and right plots correspond to absolute and normalised measurements, respectively. The lower panel in each plot shows the ratios of the theoretical predictions to the data. The dark and light bands show the relative statistical and total uncertainties in the data, respectively. 

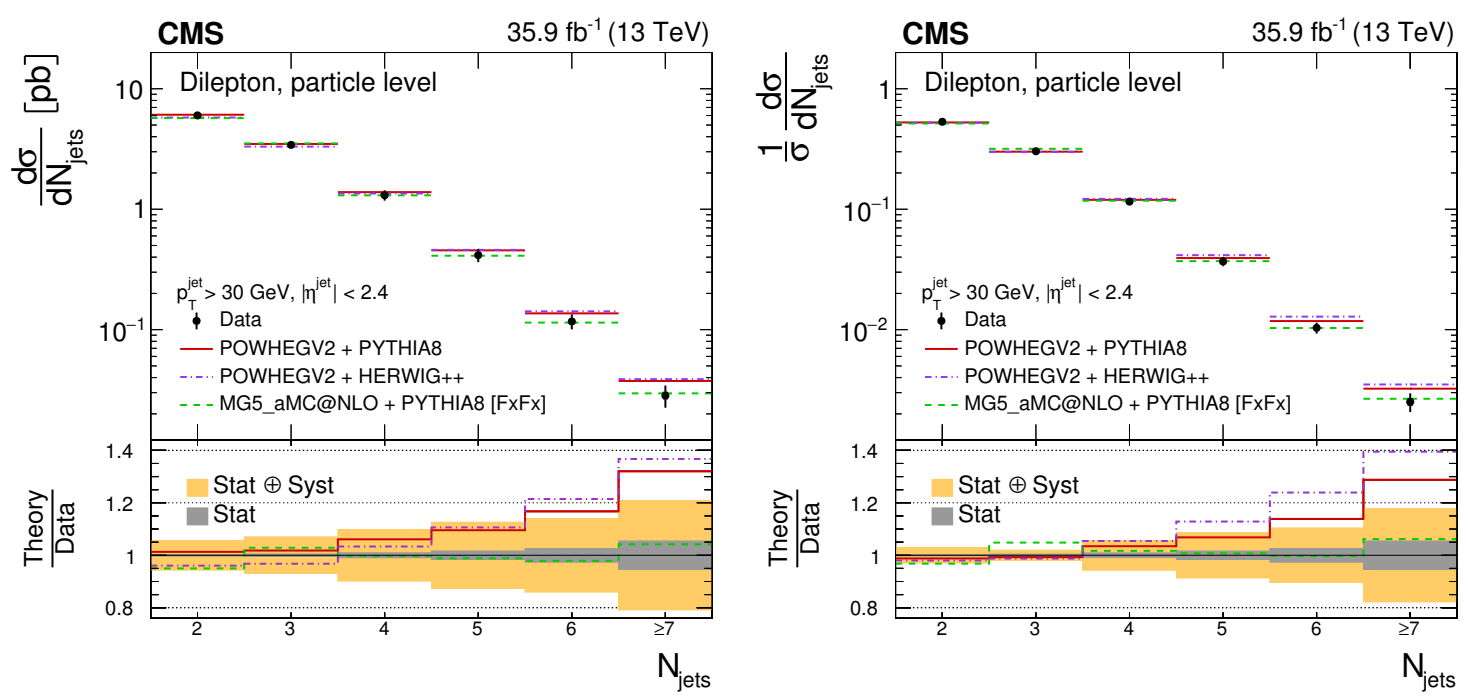

Figure 51. The differential t $\overline{\mathrm{t}}$ production cross sections as a function of $N_{\text {jets }}$ in a fiducial phase space at the particle level are shown for the data (points) and the MC predictions (lines). The vertical lines on the points indicate the total uncertainty in the data. The left and right plots correspond to absolute and normalised measurements, respectively. The lower panel in each plot shows the ratios of the theoretical predictions to the data. The dark and light bands show the relative statistical and total uncertainties in the data, respectively.

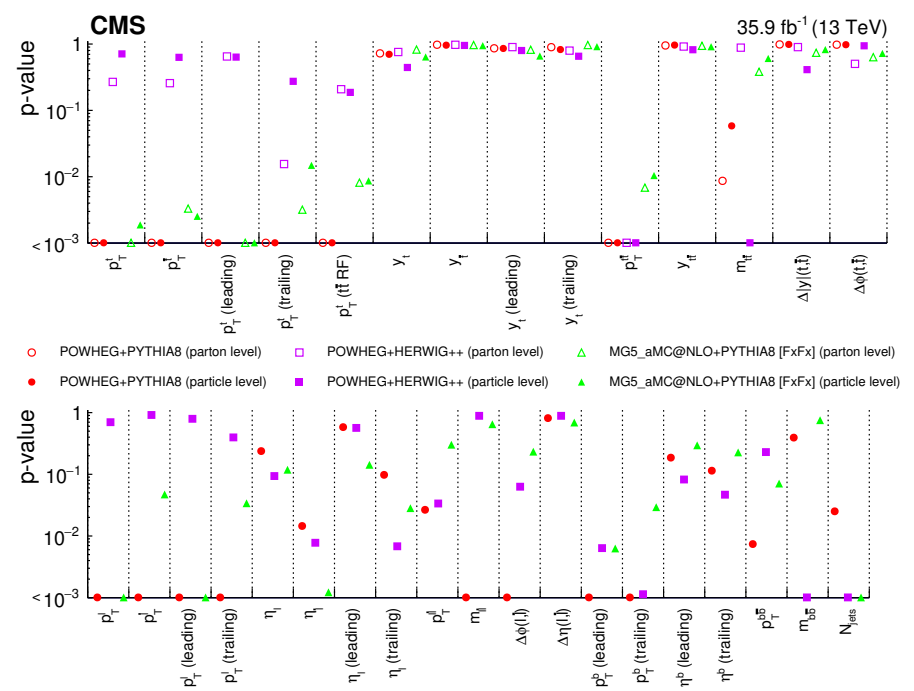

Figure 52. The $p$-values quantifying the agreement between the data and MC predictions from for all normalised measurements are shown. Points situated on the horizontal axis indicate $p$-values less than 0.001. The upper panel includes distributions measured at parton and particle levels while the bottom panel includes those measured at particle level only. 


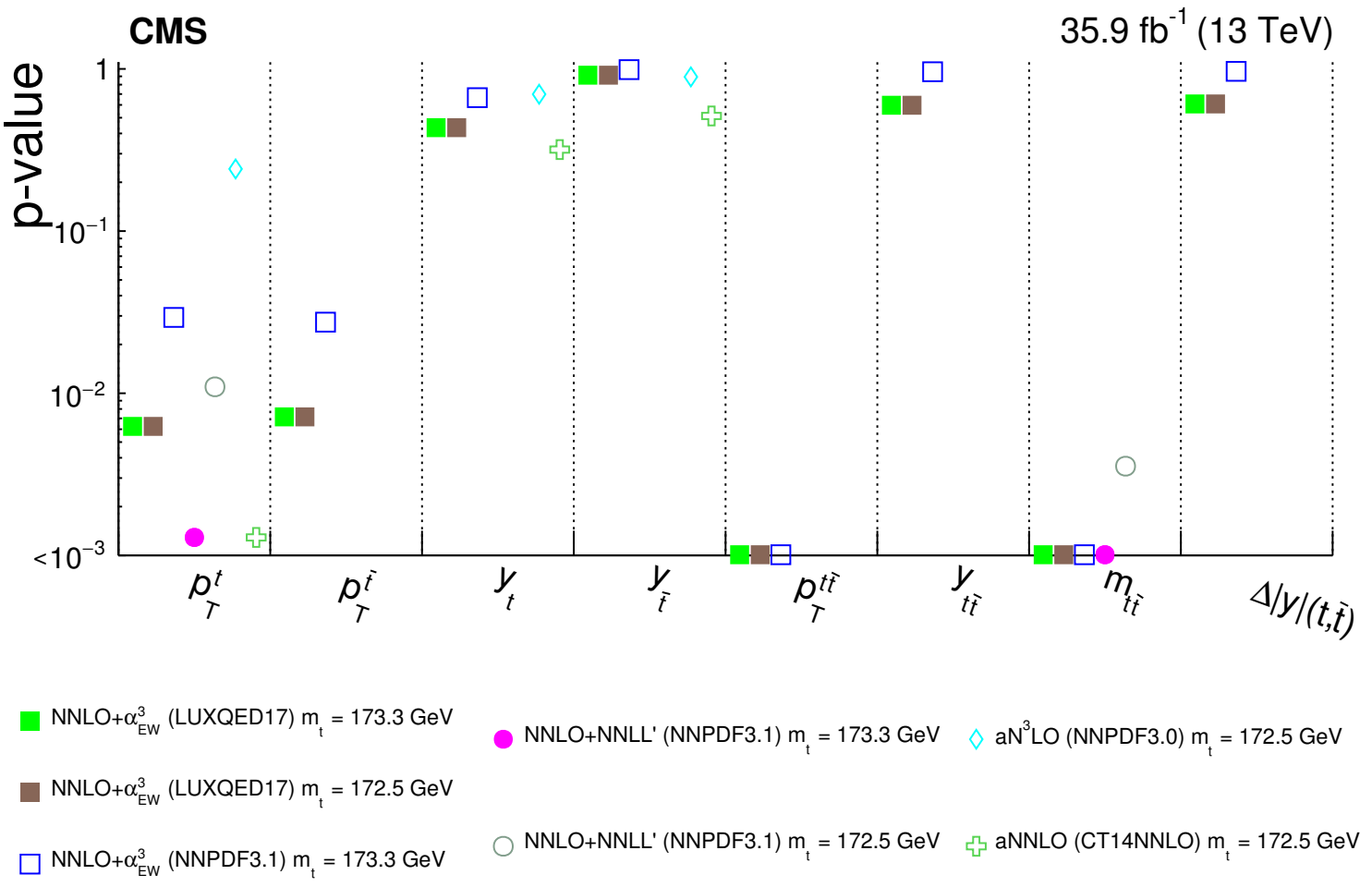

Figure 53. The $p$-values quantifying the agreement between theoretical predictions with beyondNLO precision and the data for selected normalised measurements at parton level are shown. Points situated on the horizontal axis indicate $p$-values of less than 0.001 .

properties of $t \bar{t}$ production. The top quark may also have an anomalous chromoelectric dipole moment, however in this analysis it is assumed to be zero following the theoretical

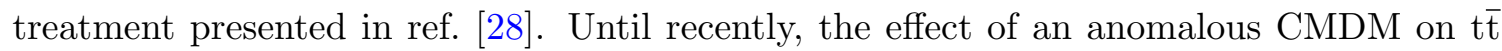
production was calculated only at LO in QCD. In ref. [28], predictions for tét production with anomalous CMDM at NLO in QCD in an EFT framework are provided. A comparison of the LO and NLO predictions reveals that the effect of the CMDM on t $\overline{\mathrm{t}}$ production is underestimated at LO, and that the NLO predictions have reduced scale uncertainties with respect to those at LO. These two factors allow stronger constraints on the CMDM to be extracted using NLO predictions than with those at LO. In an EFT framework, the scale of new physics $(\Lambda)$ is assumed to be large with respect to the typical scales probed at the LHC. Under this condition, BSM effects can be modelled in an EFT by adding a fixed set of dimension- 6 operators to the SM Lagrangian [80, 81]. An operator commonly referred to as $O_{\mathrm{tG}}$ is responsible for anomalous CMDM effects in the EFT [28]. The contribution of $O_{\mathrm{tG}}$ to the Lagrangian is parameterized by the dimensionless Wilson coefficient divided by the square of the BSM scale $\left(C_{\mathrm{tG}} / \Lambda^{2}\right)$. The $O_{\mathrm{tG}}$ operator results in a new ggt $\overline{\mathrm{t}}$ vertex, and modifies the gt $\bar{t}$ vertex, resulting in altered rates and kinematic properties in $\mathrm{t} \overline{\mathrm{t}}$ production. Furthermore, changes in the chirality of the top quarks induced by $O_{\mathrm{tG}}$ modify the spin correlation of the $t \bar{t}$ pair. Thus, both the rate of $t \bar{t}$ production and the difference in the azimuthal angle between the two leptons in dileptonic t $\overline{\mathrm{t}}$ events, $\Delta \phi(\ell, \bar{\ell})$, are sensitive to the value of $C_{\mathrm{tG}} / \Lambda^{2}$. The measurement of the absolute differential t $\overline{\mathrm{t}}$ cross section as 
a function of $\Delta \phi(\ell, \bar{\ell})$, in which the total cross section within the fiducial phase space is measured, is used to constrain $C_{\mathrm{tG}} / \Lambda^{2}$. The particle-level measurement in the fiducial phase space is the most appropriate for this purpose since it does not suffer from the model dependence introduced into the parton-level results when extrapolating to the full phase space.

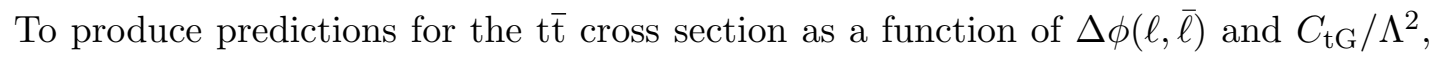
the model described in ref. [28] is implemented in the MG5_aMC@NLO generator for the ME calculation at NLO in QCD. The parton shower and hadronisation steps are performed by interfacing this setup with PYTHIA. The RIVET framework [82] is used to apply the object definitions and requirements in order to produce particle-level predictions in the fiducial phase space identical to that of the measurements presented in this paper. The normalisations of the predictions are scaled with a $K$ factor to account for the NNLO+NNLL corrections to the inclusive t $\bar{t}$ cross section calculated in ref. [83]. However, as the acceptance of the fiducial phase space is calculated only at NLO precision, the normalisations of the predictions are not fully NNLO+NNLL precise. Since the $p_{\mathrm{T}}^{\mathrm{t}}$ distribution is poorly modelled by the NLO generators, the predictions are additionally corrected in order to match the $p_{\mathrm{T}}^{\mathrm{t}}$ prediction provided in ref. [74] that corresponds to NNLO precision in QCD and includes electroweak corrections up to $\alpha_{\mathrm{EW}}^{3}$.

The upper left plot of figure 54, shows the measured differential cross section as a function of $\Delta \phi(\ell, \bar{\ell})$ along with theoretical predictions for $C_{\mathrm{tG}} / \Lambda^{2}$ values of $1.0,0.0$, and $-1.0 \mathrm{TeV}^{-2}$. The high sensitivity of the normalisation of the measured differential cross section and the smaller sensitivity of its shape to the value of $C_{\mathrm{tG}} / \Lambda^{2}$ are clearly seen in the lower panel of the left plot of figure 54 , which displays the ratios of the predictions to the measurements for the three $C_{\mathrm{tG}} / \Lambda^{2}$ values. The good agreement between the data and the $C_{\mathrm{tG}} / \Lambda^{2}=0.0 \mathrm{TeV}^{-2}$ prediction corresponding to the $\mathrm{SM}$ is also apparent.

A $\chi^{2}$ minimisation technique is used to constrain $C_{\mathrm{tG}} / \Lambda^{2}$. The $\chi^{2}$ function is defined as:

$$
\chi^{2}\left(C_{\mathrm{tG}} / \Lambda^{2}\right)=\sum_{i=1}^{N} \sum_{j=1}^{N}\left(\operatorname{data}_{i}-\operatorname{pred}_{i}\left(C_{\mathrm{tG}} / \Lambda^{2}\right)\right)\left(\operatorname{data}_{j}-\operatorname{pred}_{j}\left(C_{\mathrm{tG}} / \Lambda^{2}\right)\right) \operatorname{Cov}_{i, j}^{-1},
$$

where $\operatorname{data}_{i}$ and $\operatorname{pred}_{i}\left(C_{\mathrm{tG}} / \Lambda^{2}\right)$ are the measured and predicted differential cross section in the $i$ th bin, respectively, and $\operatorname{Cov}_{i, j}^{-1}$ is the ( $i$ th, $j$ th) element of the inverse of the covariance matrix of the data. The covariance matrix accounts for all systematic and statistical uncertainties, as well as the inter-bin correlations introduced in the unfolding process. The minimisation results in a best fit value of $0.18 \mathrm{TeV}^{-2}$, corresponding to a $\chi^{2} /$ dof of 0.3. Assuming Gaussian probability density functions for the uncertainties in the unfolded data, confidence intervals (CIs) can be estimated from the values of $C_{\mathrm{tG}} / \Lambda^{2}$ for which the $\Delta \chi^{2}$ reaches certain values. The $\Delta \chi^{2}$ is defined as the difference in $\chi^{2}$ from the $\chi^{2}$ at the best fit value. This procedure yields a $95 \%$ CI of $-0.06<C_{\mathrm{tG}} / \Lambda^{2}<$ $0.41 \mathrm{TeV}^{-2}$. Uncertainties arising from the theoretical predictions are separately estimated. First, the normalisations of the predictions are varied by $+5.8 \%$ and $-6.2 \%$, corresponding to the addition in quadrature of the uncertainties from variations of the factorisation and renormalisation scales, PDFs, and $m_{\mathrm{t}}$ in the prediction from ref. [83]. Second, the shapes 

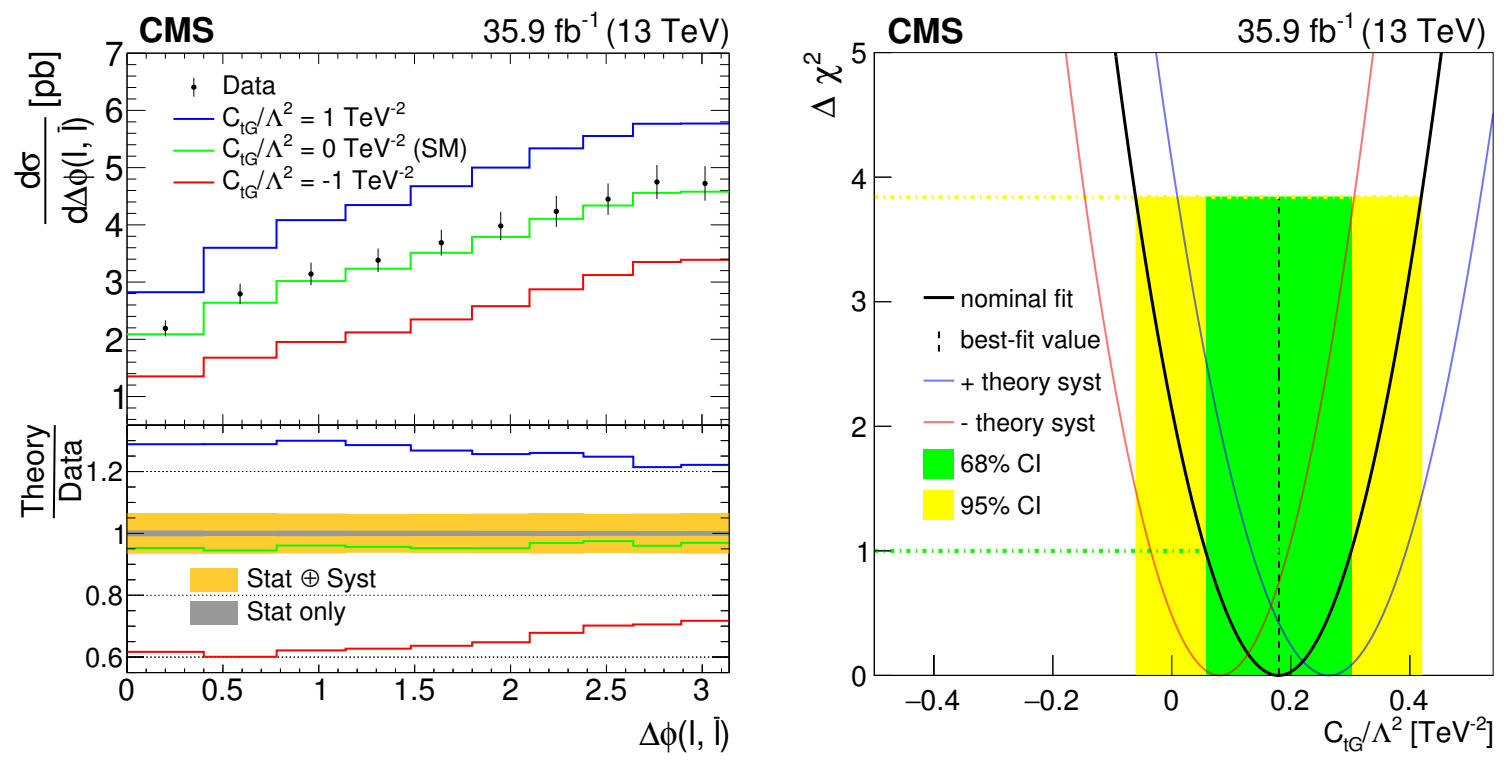

Figure 54. In the left plot, the differential t $\bar{t}$ cross sections as a function of $\Delta \phi(\ell, \bar{\ell})$ at the particle level in a fiducial phase space described in the text are shown. The points correspond to data and vertical bars on the points give the total uncertainty. The solid lines show the NLO predictions from the MG5_aMC@NLO generator interfaced with PYTHIA for $C_{\mathrm{tG}} / \Lambda^{2}$ values of 1.0, 0.0, and $-1.0 \mathrm{TeV}^{-2}$. The lower plot displays the ratio of the theoretical predictions to the data. In the right plot, $\Delta \chi^{2}$ values from the fit to the data in the left plot are shown as a function of $C_{\mathrm{tG}} / \Lambda^{2}$. The dark curve gives the result of the nominal fit, with the vertical dashed line giving the best-fit value. The two horizontal dashed lines indicate the $\Delta \chi^{2}$ values for the 68 and $95 \%$ CIs. The dark and light bands correspond to those 68 and $95 \%$ CIs, respectively. The other curves show the $\Delta \chi^{2}$ values for fits that give the maximally positive and negative changes in the best-fit value when the theoretical predictions are allowed to vary within their systematic uncertainties.

of the predictions are varied by changing the factorisation and renormalisation scales by factors of 0.5 and 2.0 in the MG5_aMC@NLO simulation. The $\chi^{2}$ minimisation is repeated for all variations, and the total theoretical uncertainty is determined from the maximally positive and negative effects on the best fit value of $C_{\mathrm{tG}} / \Lambda^{2}$. In the right plot of figure 54, the $\Delta \chi^{2}$ as a function of $C_{\mathrm{tG}} / \Lambda^{2}$ is shown. The nominal fit to the data is represented by the solid curve with the $\Delta \chi^{2}$ values for the 68 and $95 \%$ CIs indicated by the horizontal dashed lines. The dark and light regions display the corresponding 68 and $95 \%$ CIs, respectively. Since the theoretical uncertainties do not have a clear frequentist interpretation, they are not included in the CIs. Rather, the other two curves in the figure show the results of the fits that produce the maximally positive and negative deviations from the best-fit value when the theoretical predictions are allowed to vary within their uncertainties.

In ref. [28], $95 \%$ CIs of $-0.42<C_{\mathrm{tG}} / \Lambda^{2}<0.30 \mathrm{TeV}^{-2}$ and $-0.32<C_{\mathrm{tG}} / \Lambda^{2}<$ $0.73 \mathrm{TeV}^{-2}$ are derived using NLO predictions for the total $\mathrm{t} \overline{\mathrm{t}}$ cross section as a function of $C_{\mathrm{tG}} / \Lambda^{2}$ and measurements from $\sqrt{s}=8 \mathrm{TeV}$ CMS data [84] and $\sqrt{s}=1.96 \mathrm{TeV}$ Fermilab Tevatron data [85], respectively. The CMS collaboration has previously used normalised differential $t \bar{t}$ cross sections measured in the full phase space with $8 \mathrm{TeV}$ data to constrain the top quark CMDM [86]. Using relations presented in ref. [87], these results of ref. [86] 
can be converted to a $95 \% \mathrm{CI}$ of $-0.89<C_{\mathrm{tG}} / \Lambda^{2}<0.43 \mathrm{TeV}^{-2}$. Thus, the results of this work are consistent with, and improve upon, these previous constraints on $C_{\mathrm{tG}} / \Lambda^{2}$.

\section{Extraction of the top quark charge asymmetries}

The measurements of normalised differential cross sections as a function of $\Delta|y|(\mathrm{t}, \overline{\mathrm{t}})$ at parton and particle levels, and as a function of $\Delta \eta(\ell, \bar{\ell})$ at particle level shown in figures 20 and 44 , respectively, allow the extraction of the $\mathrm{t} \overline{\mathrm{t}}$ and leptonic charge asymmetries, $A_{\mathrm{c}}^{\mathrm{t} \overline{\mathrm{t}}}$ and $A_{\mathrm{c}}^{\ell \bar{\ell}}$. These observables are sensitive to a number of BSM scenarios such as axigluon, $\mathrm{Z}^{\prime}$, and $\mathrm{W}^{\prime}$ states coupling to top quarks [88]. The $A_{\mathrm{c}}^{\mathrm{t} \overline{\mathrm{t}}}$ and $A_{\mathrm{c}}^{\ell \bar{\ell}}$ asymmetries are defined as:

$$
A_{\mathrm{c}}^{\mathrm{t} \overline{\mathrm{t}}}=\frac{\sigma_{\mathrm{t} \overline{\mathrm{t}}}(\Delta|y|(\mathrm{t}, \overline{\mathrm{t}})>0)-\sigma_{\mathrm{t} \overline{\mathrm{t}}}(\Delta|y|(\mathrm{t}, \overline{\mathrm{t}})<0)}{\sigma_{\mathrm{t} \overline{\mathrm{t}}}(\Delta|y|(\mathrm{t}, \overline{\mathrm{t}})>0)+\sigma_{\mathrm{t} \overline{\mathrm{t}}}(\Delta|y|(\mathrm{t}, \overline{\mathrm{t}})<0)}, \quad A_{\mathrm{c}}^{q \bar{\ell}}=\frac{\sigma_{\mathrm{t} \overline{\mathrm{t}}}(\Delta \eta(\ell, \bar{\ell})>0)-\sigma_{\mathrm{t} \overline{\mathrm{t}}}(\Delta \eta(\ell, \bar{\ell})<0)}{\sigma_{\mathrm{t} \overline{\mathrm{t}}}(\Delta \eta(\ell, \bar{\ell})>0)+\sigma_{\mathrm{t} \overline{\mathrm{t}}}(\Delta \eta(\ell, \bar{\ell})<0)}
$$

where $\sigma_{\mathrm{t} \overline{\mathrm{t}}}$ represents the measured integrated $\mathrm{t} \overline{\mathrm{t}}$ cross section in the specified range [89]. After the extraction of $A_{\mathrm{c}}^{\mathrm{t} \overline{\mathrm{t}}}$ and $A_{\mathrm{c}}^{\ell \bar{\ell}}$ from the data, the uncertainties in $A_{\mathrm{c}}^{\mathrm{t} \overline{\mathrm{t}}}$ and $A_{\mathrm{c}}^{\ell \bar{\ell}}$ are derived by combining the statistical and systematic uncertainties in the data in each bin, while accounting for the inter-bin correlations introduced during the unfolding procedure. The measured charge asymmetries and corresponding uncertainties are: $A_{\mathrm{c}}^{\mathrm{t \textrm {t }}}($ parton level) $=$ $0.01 \pm 0.009, A_{\mathrm{c}}^{\mathrm{t \overline { \textrm {t } }}}($ particle level $)=0.008 \pm 0.009$, and $A_{\mathrm{c}}^{\ell \bar{\ell}}($ particle level $)=-0.005 \pm 0.004$. In figure 55, the central values and the 68 and $95 \%$ CI bands are compared with the SM predictions produced with the POWHEG and MG5_aMC@NLO generators interfaced with PYTHIA, and a calculation at NLO precision in QCD and including corrections arising from mixing between QCD and electroweak diagrams, and between QCD and quantum electrodynamics (QED) diagrams taken from ref. [90]. The results are in good agreement with the SM predictions and represent the first measurement of $A_{\mathrm{c}}^{\mathrm{t} \overline{\mathrm{t}}}$ and $A_{\mathrm{c}}^{\ell \bar{\ell}}$ with $13 \mathrm{TeV}$ data.

\section{Summary}

Measurements of differential t $\bar{t}$ cross sections using events containing two oppositely charged leptons produced in pp collisions at a centre-of-mass energy of $13 \mathrm{TeV}$ are presented. The data were recorded with the CMS detector in 2016 and correspond to a integrated luminosity of $35.9 \mathrm{fb}^{-1}$. The differential cross sections are presented as functions of numerous observables related to t $\overline{\mathrm{t}}$ production and decay and are based on both particle-level objects in a phase space close to that of the detector acceptance and partonlevel top quarks in the full phase space. For each observable, absolute and normalised differential cross sections are presented. Most measured differential cross sections are well modelled by theoretical predictions. However, significant disagreement between the data and Monte Carlo simulation with next-to-leading-order (NLO) precision in quantum chromodynamics is observed for the transverse momentum of top quarks, leptons, b jets, t $\bar{t}$, $\ell \bar{\ell}$, and $b \bar{b}$ systems, and the invariant mass of the $t \bar{t}, \ell \bar{\ell}$, and $b \bar{b}$ systems. Predictions with beyond-NLO precision are generally in closer agreement with the data although some significant discrepancies remain. The jet multiplicity distribution is not well described by any of the Monte Carlo predictions. The absolute particle-level differential cross section as 


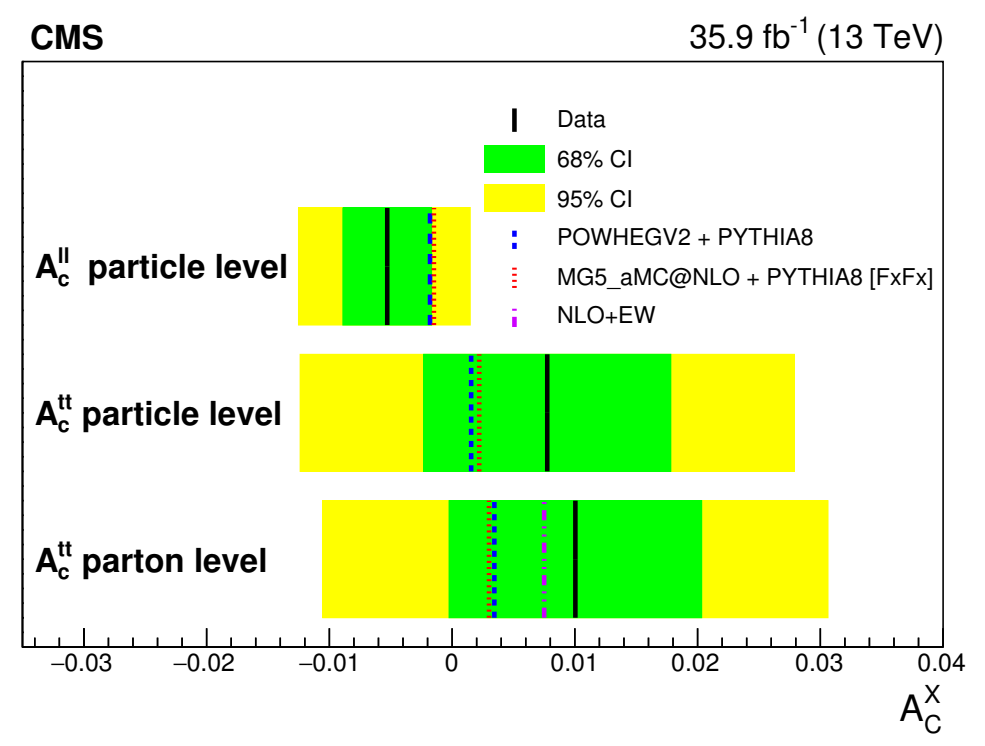

Figure 55. The results of the $A_{\mathrm{c}}^{\mathrm{x}}$ extraction $(\mathrm{x}=\mathrm{t} \overline{\mathrm{t}}$ or $\ell \bar{\ell})$ from integrating the normalised partonand particle-level differential cross section measurements as a function of $\Delta|y|(\mathrm{t}, \overline{\mathrm{t}})$ and $\Delta \eta(\ell, \bar{\ell})$ are shown. The central values for the data are indicated by the solid lines with the 68 and $95 \%$ CIs represented by the dark and light shaded bands, respectively. The three types of dashed lines indicate the SM predictions produced with the MG5_aMC@NLO and POwHEG generators, both interfaced with PYTHIA, and a calculation at NLO precision in QCD and including corrections arising from mixing between QCD and electroweak diagrams, and between QCD and QED diagrams [90].

a function of $\Delta \phi(\ell, \bar{\ell})$ is used to constrain the top quark chromomagnetic dipole moment at NLO precision in quantum chromodynamics using an effective field theory framework. The $t \bar{t}$ and leptonic charge asymmetries are measured using $13 \mathrm{TeV}$ data for the first time and found to be in agreement with standard model predictions.

\section{Acknowledgments}

We congratulate our colleagues in the CERN accelerator departments for the excellent performance of the LHC and thank the technical and administrative staffs at CERN and at other CMS institutes for their contributions to the success of the CMS effort. In addition, we gratefully acknowledge the computing centres and personnel of the Worldwide LHC Computing Grid for delivering so effectively the computing infrastructure essential to our analyses. Finally, we acknowledge the enduring support for the construction and operation of the LHC and the CMS detector provided by the following funding agencies: the Austrian Federal Ministry of Education, Science and Research and the Austrian Science Fund; the Belgian Fonds de la Recherche Scientifique, and Fonds voor Wetenschappelijk Onderzoek; the Brazilian Funding Agencies (CNPq, CAPES, FAPERJ, FAPERGS, and FAPESP); the Bulgarian Ministry of Education and Science; CERN; the Chinese Academy of Sciences, Ministry of Science and Technology, and National Natural Science Foundation of China; the Colombian Funding Agency (COLCIENCIAS); the Croatian Ministry of Science, Education and Sport, and the Croatian Science Foundation; the Research Promotion Foun- 
dation, Cyprus; the Secretariat for Higher Education, Science, Technology and Innovation, Ecuador; the Ministry of Education and Research, Estonian Research Council via IUT23-4 and IUT23-6 and European Regional Development Fund, Estonia; the Academy of Finland, Finnish Ministry of Education and Culture, and Helsinki Institute of Physics; the Institut National de Physique Nucléaire et de Physique des Particules/CNRS, and Commissariat à l'Énergie Atomique et aux Énergies Alternatives/CEA, France; the Bundesministerium für Bildung und Forschung, Deutsche Forschungsgemeinschaft, and Helmholtz-Gemeinschaft Deutscher Forschungszentren, Germany; the General Secretariat for Research and Technology, Greece; the National Research, Development and Innovation Fund, Hungary; the Department of Atomic Energy and the Department of Science and Technology, India; the Institute for Studies in Theoretical Physics and Mathematics, Iran; the Science Foundation, Ireland; the Istituto Nazionale di Fisica Nucleare, Italy; the Ministry of Science, ICT and Future Planning, and National Research Foundation (NRF), Republic of Korea; the Ministry of Education and Science of the Republic of Latvia; the Lithuanian Academy of Sciences; the Ministry of Education, and University of Malaya (Malaysia); the Ministry of Science of Montenegro; the Mexican Funding Agencies (BUAP, CINVESTAV, CONACYT, LNS, SEP, and UASLP-FAI); the Ministry of Business, Innovation and Employment, New Zealand; the Pakistan Atomic Energy Commission; the Ministry of Science and Higher Education and the National Science Centre, Poland; the Fundação para a Ciência e a Tecnologia, Portugal; JINR, Dubna; the Ministry of Education and Science of the Russian Federation, the Federal Agency of Atomic Energy of the Russian Federation, Russian Academy of Sciences, the Russian Foundation for Basic Research, and the National Research Center "Kurchatov Institute"; the Ministry of Education, Science and Technological Development of Serbia; the Secretaría de Estado de Investigación, Desarrollo e Innovación, Programa Consolider-Ingenio 2010, Plan Estatal de Investigación Científica y Técnica y de Innovación 2013-2016, Plan de Ciencia, Tecnología e Innovación 2013-2017 del Principado de Asturias, and Fondo Europeo de Desarrollo Regional, Spain; the Ministry of Science, Technology and Research, Sri Lanka; the Swiss Funding Agencies (ETH Board, ETH Zurich, PSI, SNF, UniZH, Canton Zurich, and SER); the Ministry of Science and Technology, Taipei; the Thailand Center of Excellence in Physics, the Institute for the Promotion of Teaching Science and Technology of Thailand, Special Task Force for Activating Research and the National Science and Technology Development Agency of Thailand; the Scientific and Technical Research Council of Turkey, and Turkish Atomic Energy Authority; the National Academy of Sciences of Ukraine, and State Fund for Fundamental Researches, Ukraine; the Science and Technology Facilities Council, U.K.; the U.S. Department of Energy, and the U.S. National Science Foundation.

Individuals have received support from the Marie-Curie programme and the European Research Council and Horizon 2020 Grant, contract No. 675440 (European Union); the Leventis Foundation; the A.P. Sloan Foundation; the Alexander von Humboldt Foundation; the Belgian Federal Science Policy Office; the Fonds pour la Formation à la Recherche dans l'Industrie et dans l'Agriculture (FRIA-Belgium); the Agentschap voor Innovatie door Wetenschap en Technologie (IWT-Belgium); the F.R.S.-FNRS and FWO (Belgium) under the "Excellence of Science - EOS" — be.h project n. 30820817; the Ministry of Educa- 
tion, Youth and Sports (MEYS) of the Czech Republic; the Lendület ("Momentum") Programme and the János Bolyai Research Scholarship of the Hungarian Academy of Sciences, the New National Excellence Program ÚNKP, the NKFIA research grants 123842, 123959, 124845, 124850 and 125105 (Hungary); the Council of Scientific and Industrial Research, India; the HOMING PLUS programme of the Foundation for Polish Science, cofinanced from European Union, Regional Development Fund, the Mobility Plus programme of the Ministry of Science and Higher Education, the National Science Center (Poland), contracts Harmonia 2014/14/M/ST2/00428, Opus 2014/13/B/ST2/02543, 2014/15/B/ST2/03998, and 2015/19/B/ST2/02861, Sonata-bis 2012/07/E/ST2/01406; the National Priorities Research Program by Qatar National Research Fund; the Programa de Excelencia María de Maeztu, and the Programa Severo Ochoa del Principado de Asturias; the Thalis and Aristeia programmes cofinanced by EU-ESF, and the Greek NSRF; the Rachadapisek Sompot Fund for Postdoctoral Fellowship, Chulalongkorn University, and the Chulalongkorn Academic into Its 2nd Century Project Advancement Project (Thailand); the Welch Foundation, contract C-1845; and the Weston Havens Foundation (U.S.A.).

\section{A Tables of parton-level differential cross sections}

All the measured differential cross sections at the parton level are tabulated in tables 1-14. The statistical and systematic uncertainties are quoted separately for each bin.

\begin{tabular}{|ccc|}
\hline$p_{\mathrm{T}}^{\mathrm{t}}[\mathrm{GeV}]$ & $\frac{1}{\sigma} \frac{\mathrm{d} \sigma}{\mathrm{d} p_{\mathrm{T}}^{\mathrm{t}}}\left[\mathrm{GeV}^{-1}\right]$ & $\frac{\mathrm{d} \sigma}{\mathrm{d} p_{\mathrm{T}}^{\mathrm{t}}}[\mathrm{pb} / \mathrm{GeV}]$ \\
\hline$[0,65]$ & $(4.118 \pm 0.044 \pm 0.194) \times 10^{-3}$ & $3.487 \pm 0.039 \pm 0.278$ \\
{$[65,125]$} & $(6.016 \pm 0.059 \pm 0.282) \times 10^{-3}$ & $5.094 \pm 0.05 \pm 0.467$ \\
{$[125,200]$} & $(3.352 \pm 0.03 \pm 0.116) \times 10^{-3}$ & $2.838 \pm 0.026 \pm 0.211$ \\
{$[200,290]$} & $(9.948 \pm 0.125 \pm 0.386) \times 10^{-4}$ & $0.842 \pm 0.011 \pm 0.051$ \\
{$[290,400]$} & $(2.213 \pm 0.035 \pm 0.106) \times 10^{-4}$ & $0.187 \pm 0.003 \pm 0.013$ \\
{$[400,550]$} & $(4.074 \pm 0.155 \pm 0.296) \times 10^{-5}$ & $(3.45 \pm 0.131 \pm 0.302) \times 10^{-2}$ \\
\hline
\end{tabular}

Table 1. The measured differential cross section and bin boundaries for each bin of the normalized and absolute measurements of the $t \bar{t}$ differential cross section at parton level in the full phase space as a function of $p_{\mathrm{T}}^{\mathrm{t}}$ are tabulated. 


\begin{tabular}{|ccc|}
\hline$p_{\mathrm{T}}^{\mathrm{\tau}}[\mathrm{GeV}]$ & $\frac{1}{\sigma} \frac{\mathrm{d} \sigma}{\mathrm{d} p_{\mathrm{T}}^{\mathrm{t}}}\left[\mathrm{GeV}^{-1}\right]$ & $\frac{\mathrm{d} \sigma}{\mathrm{d} p_{\mathrm{T}}^{\mathrm{t}}}[\mathrm{pb} / \mathrm{GeV}]$ \\
\hline$[0,65]$ & $(4.172 \pm 0.044 \pm 0.244) \times 10^{-3}$ & $3.532 \pm 0.039 \pm 0.313$ \\
{$[65,125]$} & $(6.031 \pm 0.059 \pm 0.224) \times 10^{-3}$ & $5.105 \pm 0.05 \pm 0.436$ \\
{$[125,200]$} & $(3.254 \pm 0.03 \pm 0.12) \times 10^{-3}$ & $2.755 \pm 0.026 \pm 0.212$ \\
{$[200,290]$} & $(1.027 \pm 0.013 \pm 0.051) \times 10^{-3}$ & $0.869 \pm 0.011 \pm 0.059$ \\
{$[290,400]$} & $(2.239 \pm 0.035 \pm 0.11) \times 10^{-4}$ & $0.19 \pm 0.003 \pm 0.012$ \\
{$[400,550]$} & $(3.941 \pm 0.152 \pm 0.506) \times 10^{-5}$ & $(3.336 \pm 0.129 \pm 0.466) \times 10^{-2}$ \\
\hline
\end{tabular}

Table 2. The measured differential cross section and bin boundaries for each bin of the normalized and absolute measurements of the $t \bar{t}$ differential cross section at parton level in the full phase space as a function of $p_{\mathrm{T}}^{\overline{\mathrm{t}}}$ are tabulated.

\begin{tabular}{|ccc|}
\hline$p_{\mathrm{T}}^{\mathrm{t}}($ leading $)[\mathrm{GeV}]$ & $\frac{1}{\sigma} \frac{\mathrm{d} \sigma}{\mathrm{d} p_{\mathrm{T}}^{\mathrm{t}}(\text { leading })}\left[\mathrm{GeV}^{-1}\right]$ & $\frac{\mathrm{d} \sigma}{\left.\mathrm{d} p_{\mathrm{T}}^{\mathrm{t}} \text { leading }\right)}[\mathrm{pb} / \mathrm{GeV}]$ \\
\hline$[0,65]$ & $(2.618 \pm 0.033 \pm 0.162) \times 10^{-3}$ & $2.221 \pm 0.029 \pm 0.195$ \\
{$[65,125]$} & $(6.046 \pm 0.042 \pm 0.155) \times 10^{-3}$ & $5.129 \pm 0.037 \pm 0.403$ \\
{$[125,200]$} & $(3.981 \pm 0.027 \pm 0.111) \times 10^{-3}$ & $3.377 \pm 0.024 \pm 0.265$ \\
{$[200,290]$} & $(1.377 \pm 0.013 \pm 0.044) \times 10^{-3}$ & $1.168 \pm 0.011 \pm 0.071$ \\
{$[290,400]$} & $(3.227 \pm 0.038 \pm 0.124) \times 10^{-4}$ & $0.274 \pm 0.003 \pm 0.017$ \\
{$[400,550]$} & $(6.076 \pm 0.171 \pm 0.439) \times 10^{-5}$ & $(5.154 \pm 0.145 \pm 0.466) \times 10^{-2}$ \\
\hline
\end{tabular}

Table 3. The measured differential cross section and bin boundaries for each bin of the normalized

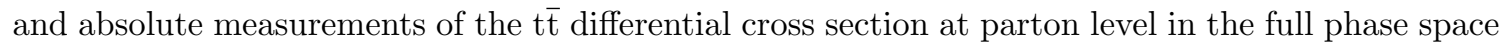
as a function of $p_{\mathrm{T}}^{\mathrm{t}}$ (leading) are tabulated.

\begin{tabular}{|ccc|}
\hline$p_{\mathrm{T}}^{\mathrm{t}}$ (trailing) $[\mathrm{GeV}]$ & $\frac{1}{\sigma} \frac{\mathrm{d} \sigma}{\mathrm{d} p_{\mathrm{T}}^{\mathrm{t}}(\text { trailing })}\left[\mathrm{GeV}^{-1}\right]$ & $\frac{\mathrm{d} \sigma}{\mathrm{d} p_{\mathrm{T}}^{\mathrm{t}} \text { (trailing) }}[\mathrm{pb} / \mathrm{GeV}]$ \\
\hline$[0,65]$ & $(5.659 \pm 0.048 \pm 0.232) \times 10^{-3}$ & $4.79 \pm 0.043 \pm 0.371$ \\
{$[65,125]$} & $(5.992 \pm 0.068 \pm 0.289) \times 10^{-3}$ & $5.071 \pm 0.058 \pm 0.486$ \\
{$[125,200]$} & $(2.641 \pm 0.032 \pm 0.125) \times 10^{-3}$ & $2.235 \pm 0.027 \pm 0.162$ \\
{$[200,290]$} & $(6.452 \pm 0.121 \pm 0.441) \times 10^{-4}$ & $0.546 \pm 0.01 \pm 0.043$ \\
{$[290,400]$} & $(1.235 \pm 0.03 \pm 0.083) \times 10^{-4}$ & $0.105 \pm 0.003 \pm 0.009$ \\
{$[400,550]$} & $(1.949 \pm 0.126 \pm 0.306) \times 10^{-5}$ & $(1.65 \pm 0.107 \pm 0.267) \times 10^{-2}$ \\
\hline
\end{tabular}

Table 4. The measured differential cross section and bin boundaries for each bin of the normalized and absolute measurements of the $t \bar{t}$ differential cross section at parton level in the full phase space as a function of $p_{\mathrm{T}}^{\mathrm{t}}$ (trailing) are tabulated. 


\begin{tabular}{|ccc|}
\hline$p_{\mathrm{T}}^{\mathrm{t}}(\mathrm{t \overline { \textrm {t } } \mathrm { RF }})[\mathrm{GeV}]$ & $\frac{1}{\sigma} \frac{\mathrm{d} \sigma}{\mathrm{d} p_{\mathrm{T}}^{\mathrm{t}}(\mathrm{t} \mathrm{RF})}\left[\mathrm{GeV}^{-1}\right]$ & $\frac{\mathrm{d} \sigma}{\mathrm{d} p_{\mathrm{T}}^{\mathrm{t}}(\mathrm{t \overline { \textrm {t } } \mathrm { RF }})}[\mathrm{pb} / \mathrm{GeV}]$ \\
\hline$[0,65]$ & $(4.505 \pm 0.045 \pm 0.218) \times 10^{-3}$ & $3.814 \pm 0.041 \pm 0.296$ \\
{$[65,125]$} & $(6.24 \pm 0.065 \pm 0.263) \times 10^{-3}$ & $5.282 \pm 0.056 \pm 0.489$ \\
{$[125,200]$} & $(3.149 \pm 0.032 \pm 0.102) \times 10^{-3}$ & $2.666 \pm 0.028 \pm 0.191$ \\
{$[200,290]$} & $(8.229 \pm 0.126 \pm 0.405) \times 10^{-4}$ & $0.697 \pm 0.011 \pm 0.045$ \\
{$[290,400]$} & $(1.682 \pm 0.032 \pm 0.091) \times 10^{-4}$ & $0.142 \pm 0.003 \pm 0.009$ \\
{$[400,550]$} & $(2.64 \pm 0.136 \pm 0.284) \times 10^{-5}$ & $(2.235 \pm 0.115 \pm 0.266) \times 10^{-2}$ \\
\hline
\end{tabular}

Table 5. The measured differential cross section and bin boundaries for each bin of the normalized

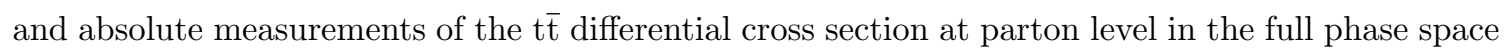
as a function of $p_{\mathrm{T}}^{\mathrm{t}}(\mathrm{t} \overline{\mathrm{t}} \mathrm{RF})$ are tabulated.

\begin{tabular}{|ccc|}
\hline$y_{\mathrm{t}}$ & $\frac{1}{\sigma} \frac{\mathrm{d} \sigma}{\mathrm{d} y_{\mathrm{t}}}$ & $\frac{\mathrm{d} \sigma}{\mathrm{d} y_{\mathrm{t}}}[\mathrm{pb}]$ \\
\hline$[-2.6,-1.8]$ & $(7.371 \pm 0.137 \pm 0.395) \times 10^{-2}$ & $(6 \pm 0.117 \pm 0.614) \times 10$ \\
{$[-1.8,-1.35]$} & $0.162 \pm 0.002 \pm 0.004$ & $(1.32 \pm 0.014 \pm 0.098) \times 10^{2}$ \\
{$[-1.35,-0.9]$} & $0.231 \pm 0.002 \pm 0.006$ & $(1.884 \pm 0.019 \pm 0.131) \times 10^{2}$ \\
{$[-0.9,-0.45]$} & $0.279 \pm 0.003 \pm 0.006$ & $(2.274 \pm 0.022 \pm 0.157) \times 10^{2}$ \\
{$[-0.45,0]$} & $0.301 \pm 0.003 \pm 0.009$ & $(2.452 \pm 0.023 \pm 0.172) \times 10^{2}$ \\
{$[0,0.45]$} & $0.304 \pm 0.003 \pm 0.011$ & $(2.474 \pm 0.023 \pm 0.191) \times 10^{2}$ \\
{$[0.45,0.9]$} & $0.286 \pm 0.003 \pm 0.009$ & $(2.326 \pm 0.021 \pm 0.163) \times 10^{2}$ \\
{$[0.9,1.35]$} & $0.227 \pm 0.002 \pm 0.005$ & $(1.844 \pm 0.018 \pm 0.131) \times 10^{2}$ \\
{$[1.35,1.8]$} & $0.164 \pm 0.002 \pm 0.005$ & $(1.331 \pm 0.014 \pm 0.103) \times 10^{2}$ \\
{$[1.8,2.6]$} & $(7.737 \pm 0.135 \pm 0.328) \times 10^{-2}$ & $(6.298 \pm 0.116 \pm 0.561) \times 10$ \\
\hline
\end{tabular}

Table 6. The measured differential cross section and bin boundaries for each bin of the normalized and absolute measurements of the t $\overline{\mathrm{t}}$ differential cross section at parton level in the full phase space as a function of $y_{\mathrm{t}}$ are tabulated. 


\begin{tabular}{|ccc|}
\hline$y_{\overline{\mathrm{t}}}$ & $\frac{1}{\sigma} \frac{\mathrm{d} \sigma}{\mathrm{d} y_{\overline{\mathrm{t}}}}$ & $\frac{\mathrm{d} \sigma}{\mathrm{d} y_{\overline{\mathrm{t}}}}[\mathrm{pb}]$ \\
\hline$[-2.6,-1.8]$ & $(7.496 \pm 0.137 \pm 0.35) \times 10^{-2}$ & $(6.106 \pm 0.117 \pm 0.587) \times 10$ \\
{$[-1.8,-1.35]$} & $0.159 \pm 0.002 \pm 0.003$ & $(1.299 \pm 0.014 \pm 0.099) \times 10^{2}$ \\
{$[-1.35,-0.9]$} & $0.231 \pm 0.002 \pm 0.006$ & $(1.881 \pm 0.019 \pm 0.129) \times 10^{2}$ \\
{$[-0.9,-0.45]$} & $0.279 \pm 0.003 \pm 0.007$ & $(2.273 \pm 0.022 \pm 0.157) \times 10^{2}$ \\
{$[-0.45,0]$} & $0.307 \pm 0.003 \pm 0.006$ & $(2.499 \pm 0.023 \pm 0.18) \times 10^{2}$ \\
{$[0,0.45]$} & $0.307 \pm 0.003 \pm 0.009$ & $(2.501 \pm 0.023 \pm 0.171) \times 10^{2}$ \\
{$[0.45,0.9]$} & $0.277 \pm 0.003 \pm 0.006$ & $(2.255 \pm 0.021 \pm 0.155) \times 10^{2}$ \\
{$[0.9,1.35]$} & $0.236 \pm 0.002 \pm 0.009$ & $(1.921 \pm 0.018 \pm 0.141) \times 10^{2}$ \\
{$[1.35,1.8]$} & $0.161 \pm 0.002 \pm 0.009$ & $(1.307 \pm 0.014 \pm 0.12) \times 10^{2}$ \\
{$[1.8,2.6]$} & $(7.454 \pm 0.135 \pm 0.428) \times 10^{-2}$ & $(6.072 \pm 0.116 \pm 0.602) \times 10$ \\
\hline
\end{tabular}

Table 7. The measured differential cross section and bin boundaries for each bin of the normalized

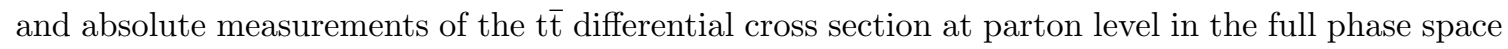
as a function of $y_{\overline{\mathrm{t}}}$ are tabulated.

\begin{tabular}{|ccc|}
\hline$y_{\mathrm{t}}($ leading $)$ & $\frac{1}{\sigma} \frac{\mathrm{d} \sigma}{\mathrm{d} y_{\mathrm{t}} \text { (leading) }}$ & $\frac{\mathrm{d} \sigma}{\left.\mathrm{d} y_{\mathrm{t}} \text { (leading }\right)}[\mathrm{pb}]$ \\
\hline$[-2.6,-1.65]$ & $(8.214 \pm 0.137 \pm 0.385) \times 10^{-2}$ & $(6.707 \pm 0.119 \pm 0.65) \times 10$ \\
{$[-1.65,-1.1]$} & $0.191 \pm 0.002 \pm 0.005$ & $(1.56 \pm 0.015 \pm 0.119) \times 10^{2}$ \\
{$[-1.1,-0.55]$} & $0.27 \pm 0.002 \pm 0.007$ & $(2.208 \pm 0.018 \pm 0.147) \times 10^{2}$ \\
{$[-0.55,0]$} & $0.303 \pm 0.003 \pm 0.007$ & $(2.473 \pm 0.021 \pm 0.168) \times 10^{2}$ \\
{$[0,0.55]$} & $0.304 \pm 0.003 \pm 0.007$ & $(2.481 \pm 0.021 \pm 0.167) \times 10^{2}$ \\
{$[0.55,1.1]$} & $0.27 \pm 0.002 \pm 0.006$ & $(2.206 \pm 0.019 \pm 0.147) \times 10^{2}$ \\
{$[1.1,1.65]$} & $0.189 \pm 0.002 \pm 0.008$ & $(1.546 \pm 0.015 \pm 0.131) \times 10^{2}$ \\
{$[1.65,2.6]$} & $(8.621 \pm 0.131 \pm 0.484) \times 10^{-2}$ & $(7.04 \pm 0.114 \pm 0.674) \times 10$ \\
\hline
\end{tabular}

Table 8. The measured differential cross section and bin boundaries for each bin of the normalized

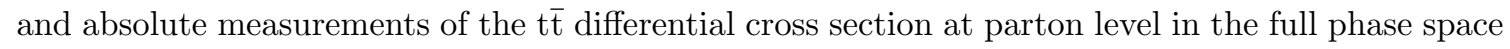
as a function of $y_{\mathrm{t}}$ (leading) are tabulated. 


\begin{tabular}{|ccc|}
\hline$y_{\mathrm{t}}$ (trailing) & $\frac{1}{\sigma} \frac{\mathrm{d} \sigma}{\left.\mathrm{d} y_{\mathrm{t}} \text { (trailing }\right)}$ & $\frac{\mathrm{d} \sigma}{\left.\mathrm{d} y_{\mathrm{t}} \text { (trailing }\right)}[\mathrm{pb}]$ \\
\hline$[-2.6,-1.65]$ & $(8.68 \pm 0.152 \pm 0.446) \times 10^{-2}$ & $(7.056 \pm 0.131 \pm 0.73) \times 10$ \\
{$[-1.65,-1.1]$} & $0.191 \pm 0.002 \pm 0.006$ & $(1.552 \pm 0.016 \pm 0.119) \times 10^{2}$ \\
{$[-1.1,-0.55]$} & $0.265 \pm 0.003 \pm 0.009$ & $(2.158 \pm 0.02 \pm 0.146) \times 10^{2}$ \\
{$[-0.55,0]$} & $0.299 \pm 0.003 \pm 0.007$ & $(2.427 \pm 0.022 \pm 0.174) \times 10^{2}$ \\
{$[0,0.55]$} & $0.297 \pm 0.003 \pm 0.012$ & $(2.418 \pm 0.023 \pm 0.19) \times 10^{2}$ \\
{$[0.55,1.1]$} & $0.27 \pm 0.003 \pm 0.008$ & $(2.194 \pm 0.021 \pm 0.151) \times 10^{2}$ \\
{$[1.1,1.65]$} & $0.197 \pm 0.002 \pm 0.006$ & $(1.598 \pm 0.016 \pm 0.119) \times 10^{2}$ \\
{$[1.65,2.6]$} & $(8.644 \pm 0.144 \pm 0.387) \times 10^{-2}$ & $(7.027 \pm 0.125 \pm 0.62) \times 10$ \\
\hline
\end{tabular}

Table 9. The measured differential cross section and bin boundaries for each bin of the normalized and absolute measurements of the $t \bar{t}$ differential cross section at parton level in the full phase space as a function of $y_{\mathrm{t}}$ (trailing) are tabulated.

\begin{tabular}{|c|c|c|}
\hline$p_{\mathrm{T}}^{\mathrm{t} \overline{\mathrm{t}}}$ & $\frac{1}{\sigma} \frac{\mathrm{d} \sigma}{\mathrm{d} p_{\mathrm{T}}^{\mathrm{t} \overline{\mathrm{t}}}}$ & $\frac{\mathrm{d} \sigma}{\mathrm{d} p_{\mathrm{T}}^{\mathrm{t \overline {t }}}}[\mathrm{pb}]$ \\
\hline$[0,40]$ & $(1.275 \pm 0.006 \pm 0.085) \times 10^{-2}$ & $(1.074 \pm 0.006 \pm 0.101) \times 10$ \\
\hline$[40,100]$ & $(4.815 \pm 0.047 \pm 0.607) \times 10^{-3}$ & $4.053 \pm 0.039 \pm 0.575$ \\
\hline$[100,200]$ & $(1.507 \pm 0.013 \pm 0.052) \times 10^{-3}$ & $1.269 \pm 0.012 \pm 0.102$ \\
\hline$[200,310]$ & $(3.368 \pm 0.049 \pm 0.126) \times 10^{-4}$ & $0.284 \pm 0.004 \pm 0.023$ \\
\hline$[310,420]$ & $(8.932 \pm 0.197 \pm 0.457) \times 10^{-5}$ & $(7.519 \pm 0.166 \pm 0.665) \times 10^{-2}$ \\
\hline$[420,570]$ & $(2.296 \pm 0.085 \pm 0.146) \times 10^{-5}$ & $(1.933 \pm 0.072 \pm 0.182) \times 10^{-2}$ \\
\hline
\end{tabular}

Table 10. The measured differential cross section and bin boundaries for each bin of the normalized

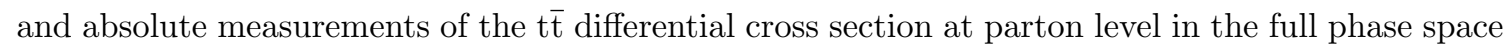
as a function of $p_{\mathrm{T}}^{\mathrm{t} \overline{\mathrm{T}}}$ are tabulated. 


\begin{tabular}{|c|c|c|}
\hline$y_{\mathrm{t} \overline{\mathrm{t}}}$ & $\frac{1}{\sigma} \frac{\mathrm{d} \sigma}{\mathrm{d} y_{\mathrm{t} \overline{\mathrm{t}}}}$ & $\frac{\mathrm{d} \sigma}{\mathrm{d} y_{\mathrm{t} \overline{\mathrm{t}}}}[\mathrm{pb}]$ \\
\hline$[-2.6,-1.6]$ & $(5.394 \pm 0.127 \pm 0.586) \times 10^{-2}$ & $(4.443 \pm 0.109 \pm 0.647) \times 10$ \\
\hline$[-1.6,-1.2]$ & $0.173 \pm 0.002 \pm 0.004$ & $(1.424 \pm 0.016 \pm 0.103) \times 10^{2}$ \\
\hline$[-1.2,-0.8]$ & $0.256 \pm 0.003 \pm 0.009$ & $(2.108 \pm 0.022 \pm 0.157) \times 10^{2}$ \\
\hline$[-0.8,-0.4]$ & $0.316 \pm 0.003 \pm 0.008$ & $(2.602 \pm 0.024 \pm 0.189) \times 10^{2}$ \\
\hline$[-0.4,0]$ & $0.369 \pm 0.003 \pm 0.01$ & $(3.04 \pm 0.026 \pm 0.191) \times 10^{2}$ \\
\hline$[0,0.4]$ & $0.353 \pm 0.003 \pm 0.008$ & $(2.91 \pm 0.025 \pm 0.209) \times 10^{2}$ \\
\hline$[0.4,0.8]$ & $0.321 \pm 0.003 \pm 0.007$ & $(2.642 \pm 0.023 \pm 0.189) \times 10^{2}$ \\
\hline$[0.8,1.2]$ & $0.261 \pm 0.002 \pm 0.008$ & $(2.149 \pm 0.02 \pm 0.148) \times 10^{2}$ \\
\hline$[1.2,1.6]$ & $0.169 \pm 0.002 \pm 0.005$ & $(1.396 \pm 0.016 \pm 0.112) \times 10^{2}$ \\
\hline$[1.6,2.6]$ & $(5.885 \pm 0.125 \pm 0.336) \times 10^{-2}$ & $(4.848 \pm 0.108 \pm 0.514) \times 10$ \\
\hline
\end{tabular}

Table 11. The measured differential cross section and bin boundaries for each bin of the normalized and absolute measurements of the t $\bar{t}$ differential cross section at parton level in the full phase space as a function of $y_{\mathrm{t} \overline{\mathrm{t}}}$ are tabulated.

\begin{tabular}{|ccc|}
\hline$m_{\mathrm{t} \overline{\mathrm{t}}}[\mathrm{GeV}]$ & $\frac{1}{\sigma} \frac{\mathrm{d} \sigma}{\mathrm{d} m_{\mathrm{t} \overline{\mathrm{t}}}}\left[\mathrm{GeV}^{-1}\right]$ & $\frac{\mathrm{d} \sigma}{\mathrm{d} m_{\mathrm{t} \overline{\mathrm{t}}}}[\mathrm{pb} / \mathrm{GeV}]$ \\
\hline$[300,380]$ & $(1.981 \pm 0.036 \pm 0.18) \times 10^{-3}$ & $1.664 \pm 0.031 \pm 0.163$ \\
{$[380,470]$} & $(3.992 \pm 0.049 \pm 0.183) \times 10^{-3}$ & $3.354 \pm 0.041 \pm 0.324$ \\
{$[470,620]$} & $(2.009 \pm 0.023 \pm 0.057) \times 10^{-3}$ & $1.688 \pm 0.019 \pm 0.122$ \\
{$[620,820]$} & $(6.363 \pm 0.108 \pm 0.355) \times 10^{-4}$ & $0.535 \pm 0.009 \pm 0.038$ \\
{$[820,1100]$} & $(1.438 \pm 0.041 \pm 0.105) \times 10^{-4}$ & $0.121 \pm 0.003 \pm 0.012$ \\
{$[1100,1500]$} & $(2.72 \pm 0.106 \pm 0.206) \times 10^{-5}$ & $(2.285 \pm 0.089 \pm 0.21) \times 10^{-2}$ \\
{$[1500,2500]$} & $(2.45 \pm 0.24 \pm 0.464) \times 10^{-6}$ & $(2.059 \pm 0.201 \pm 0.383) \times 10^{-3}$ \\
\hline
\end{tabular}

Table 12. The measured differential cross section and bin boundaries for each bin of the normalized and absolute measurements of the t $\bar{t}$ differential cross section at parton level in the full phase space as a function of $m_{\mathrm{t} \overline{\mathrm{t}}}$ are tabulated. 


\begin{tabular}{|ccc|}
\hline$\Delta|y|(\mathrm{t}, \overline{\mathrm{t}})$ & $\frac{1}{\sigma} \frac{\mathrm{d} \sigma}{\mathrm{d} \Delta|y|(\mathrm{t}, \overline{\mathrm{t}})}$ & $\frac{\mathrm{d} \sigma}{\mathrm{d} \Delta|y|(\mathrm{t}, \overline{\mathrm{t}})}[\mathrm{pb}]$ \\
\hline$[-2.6,-1.4]$ & $(5.24 \pm 0.105 \pm 0.272) \times 10^{-2}$ & $(4.365 \pm 0.09 \pm 0.408) \times 10$ \\
{$[-1.4,-0.9]$} & $0.193 \pm 0.002 \pm 0.009$ & $(1.61 \pm 0.019 \pm 0.137) \times 10^{2}$ \\
{$[-0.9,-0.4]$} & $0.321 \pm 0.003 \pm 0.007$ & $(2.675 \pm 0.028 \pm 0.202) \times 10^{2}$ \\
{$[-0.4,0]$} & $0.436 \pm 0.004 \pm 0.014$ & $(3.63 \pm 0.037 \pm 0.246) \times 10^{2}$ \\
{$[0,0.4]$} & $0.443 \pm 0.004 \pm 0.012$ & $(3.693 \pm 0.038 \pm 0.241) \times 10^{2}$ \\
{$[0.4,0.9]$} & $0.325 \pm 0.003 \pm 0.013$ & $(2.71 \pm 0.029 \pm 0.217) \times 10^{2}$ \\
{$[0.9,1.4]$} & $0.199 \pm 0.002 \pm 0.006$ & $(1.661 \pm 0.019 \pm 0.134) \times 10^{2}$ \\
{$[1.4,2.6]$} & $(5.489 \pm 0.103 \pm 0.422) \times 10^{-2}$ & $(4.572 \pm 0.088 \pm 0.47) \times 10$ \\
\hline
\end{tabular}

Table 13. The measured differential cross section and bin boundaries for each bin of the normalized

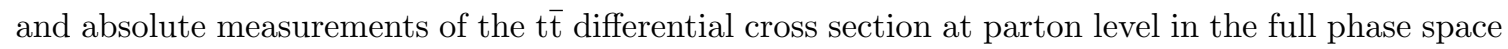
as a function of $\Delta|y|(\mathrm{t}, \overline{\mathrm{t}})$ are tabulated.

\begin{tabular}{|ccc|}
\hline$\Delta \phi(\mathrm{t}, \overline{\mathrm{t}})[\mathrm{GeV}]$ & $\frac{1}{\sigma} \frac{\mathrm{d} \sigma}{\mathrm{d} \Delta \phi(\mathrm{t}, \overline{\mathrm{t}})}\left[\mathrm{GeV}^{-1}\right]$ & $\frac{\mathrm{d} \sigma}{\mathrm{d} \Delta \phi(\mathrm{t}, \overline{\mathrm{t}})}[\mathrm{pb} / \mathrm{GeV}]$ \\
\hline$[0,1.57]$ & $(6.336 \pm 0.072 \pm 0.336) \times 10^{-2}$ & $(5.293 \pm 0.061 \pm 0.49) \times 10$ \\
{$[1.57,2.67]$} & $0.218 \pm 0.001 \pm 0.009$ & $(1.818 \pm 0.011 \pm 0.159) \times 10^{2}$ \\
{$[2.67,3.02]$} & $1.016 \pm 0.006 \pm 0.027$ & $(8.49 \pm 0.051 \pm 0.63) \times 10^{2}$ \\
{$[3.02,3.142]$} & $2.504 \pm 0.019 \pm 0.151$ & $(2.092 \pm 0.016 \pm 0.168) \times 10^{3}$ \\
\hline
\end{tabular}

Table 14. The measured differential cross section and bin boundaries for each bin of the normalized and absolute measurements of the t $\overline{\mathrm{t}}$ differential cross section at parton level in the full phase space as a function of $\Delta \phi(\mathrm{t}, \overline{\mathrm{t}})$ are tabulated.

\section{B Tables of particle-level differential cross sections}

All the measured differential cross sections at the particle level are tabulated in tables 1547. The statistical and systematic uncertainties are quoted separately for each bin. 


\begin{tabular}{|ccc|}
\hline$p_{\mathrm{T}}^{\mathrm{t}}[\mathrm{GeV}]$ & $\frac{1}{\sigma} \frac{\mathrm{d} \sigma}{\mathrm{d} p_{\mathrm{T}}^{\mathrm{t}}}\left[\mathrm{GeV}^{-1}\right]$ & $\frac{\mathrm{d} \sigma}{\mathrm{d} p_{\mathrm{T}}^{\mathrm{t}}}[\mathrm{pb} / \mathrm{GeV}]$ \\
\hline$[0,65]$ & $(3.991 \pm 0.037 \pm 0.162) \times 10^{-3}$ & $(4.529 \pm 0.043 \pm 0.328) \times 10^{-2}$ \\
{$[65,125]$} & $(5.734 \pm 0.053 \pm 0.251) \times 10^{-3}$ & $(6.507 \pm 0.061 \pm 0.538) \times 10^{-2}$ \\
{$[125,200]$} & $(3.369 \pm 0.03 \pm 0.114) \times 10^{-3}$ & $(3.823 \pm 0.035 \pm 0.255) \times 10^{-2}$ \\
{$[200,290]$} & $(1.152 \pm 0.014 \pm 0.041) \times 10^{-3}$ & $(1.307 \pm 0.016 \pm 0.077) \times 10^{-2}$ \\
{$[290,400]$} & $(2.907 \pm 0.044 \pm 0.134) \times 10^{-4}$ & $(3.299 \pm 0.051 \pm 0.236) \times 10^{-3}$ \\
{$[400,550]$} & $(5.515 \pm 0.206 \pm 0.36) \times 10^{-5}$ & $(6.259 \pm 0.234 \pm 0.555) \times 10^{-4}$ \\
\hline
\end{tabular}

Table 15. The measured differential cross section and bin boundaries for each bin of the normalized and absolute measurements of the $t \bar{t}$ differential cross section at particle level in the fiducial phase space as a function of $p_{\mathrm{T}}^{\mathrm{t}}$ are tabulated.

\begin{tabular}{|ccc|}
\hline$p_{\mathrm{T}}^{\overline{\mathrm{t}}}[\mathrm{GeV}]$ & $\frac{1}{\sigma} \frac{\mathrm{d} \sigma}{\mathrm{d} p_{\mathrm{T}}^{\mathrm{t}}}\left[\mathrm{GeV}^{-1}\right]$ & $\frac{\mathrm{d} \sigma}{\mathrm{d} p_{\mathrm{T}}^{\overline{\mathrm{t}}}}[\mathrm{pb} / \mathrm{GeV}]$ \\
\hline$[0,65]$ & $(4.038 \pm 0.037 \pm 0.205) \times 10^{-3}$ & $(4.581 \pm 0.044 \pm 0.368) \times 10^{-2}$ \\
{$[65,125]$} & $(5.746 \pm 0.053 \pm 0.192) \times 10^{-3}$ & $(6.519 \pm 0.061 \pm 0.487) \times 10^{-2}$ \\
{$[125,200]$} & $(3.277 \pm 0.03 \pm 0.107) \times 10^{-3}$ & $(3.717 \pm 0.035 \pm 0.258) \times 10^{-2}$ \\
{$[200,290]$} & $(1.186 \pm 0.014 \pm 0.053) \times 10^{-3}$ & $(1.345 \pm 0.016 \pm 0.087) \times 10^{-2}$ \\
{$[290,400]$} & $(2.937 \pm 0.045 \pm 0.122) \times 10^{-4}$ & $(3.332 \pm 0.051 \pm 0.221) \times 10^{-3}$ \\
{$[400,550]$} & $(5.37 \pm 0.204 \pm 0.672) \times 10^{-5}$ & $(6.093 \pm 0.231 \pm 0.865) \times 10^{-4}$ \\
\hline
\end{tabular}

Table 16. The measured differential cross section and bin boundaries for each bin of the normalized and absolute measurements of the t $\bar{t}$ differential cross section at particle level in the fiducial phase space as a function of $p_{\mathrm{T}}^{\overline{\mathrm{t}}}$ are tabulated.

\begin{tabular}{|ccc|}
\hline$p_{\mathrm{T}}^{\mathrm{t}}$ (leading) $[\mathrm{GeV}]$ & $\frac{1}{\sigma} \frac{\mathrm{d} \sigma}{\left.\mathrm{d} p_{\mathrm{T}}^{\mathrm{t}} \text { leading }\right)}\left[\mathrm{GeV}^{-1}\right]$ & $\frac{\mathrm{d} \sigma}{\left.\mathrm{d} p_{\mathrm{T}}^{\mathrm{t}} \text { leading }\right)}[\mathrm{pb} / \mathrm{GeV}]$ \\
\hline$[0,65]$ & $(2.474 \pm 0.028 \pm 0.136) \times 10^{-3}$ & $(2.805 \pm 0.033 \pm 0.227) \times 10^{-2}$ \\
{$[65,125]$} & $(5.735 \pm 0.041 \pm 0.145) \times 10^{-3}$ & $(6.504 \pm 0.049 \pm 0.462) \times 10^{-2}$ \\
{$[125,200]$} & $(3.937 \pm 0.027 \pm 0.102) \times 10^{-3}$ & $(4.465 \pm 0.033 \pm 0.315) \times 10^{-2}$ \\
{$[200,290]$} & $(1.568 \pm 0.015 \pm 0.046) \times 10^{-3}$ & $(1.778 \pm 0.017 \pm 0.102) \times 10^{-2}$ \\
{$[290,400]$} & $(4.199 \pm 0.049 \pm 0.141) \times 10^{-4}$ & $(4.761 \pm 0.057 \pm 0.297) \times 10^{-3}$ \\
{$[400,550]$} & $(8.319 \pm 0.232 \pm 0.532) \times 10^{-5}$ & $(9.434 \pm 0.264 \pm 0.839) \times 10^{-4}$ \\
\hline
\end{tabular}

Table 17. The measured differential cross section and bin boundaries for each bin of the normalized

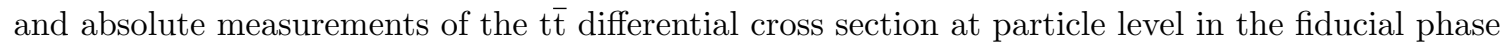
space as a function of $p_{\mathrm{T}}^{\mathrm{t}}$ (leading) are tabulated. 


\begin{tabular}{|ccc|}
\hline$p_{\mathrm{T}}^{\mathrm{t}}($ trailing $)[\mathrm{GeV}]$ & $\frac{1}{\sigma} \frac{\mathrm{d} \sigma}{\mathrm{d} p_{\mathrm{T}}^{\mathrm{t}}(\text { trailing })}\left[\mathrm{GeV}^{-1}\right]$ & $\frac{\mathrm{d} \sigma}{\left.\mathrm{d} p_{\mathrm{T}}^{\mathrm{t}} \text { (trailing }\right)}[\mathrm{pb} / \mathrm{GeV}]$ \\
\hline$[0,65]$ & $(5.535 \pm 0.039 \pm 0.193) \times 10^{-3}$ & $(6.287 \pm 0.047 \pm 0.445) \times 10^{-2}$ \\
{$[65,125]$} & $(5.737 \pm 0.058 \pm 0.244) \times 10^{-3}$ & $(6.516 \pm 0.067 \pm 0.545) \times 10^{-2}$ \\
{$[125,200]$} & $(2.726 \pm 0.032 \pm 0.114) \times 10^{-3}$ & $(3.096 \pm 0.037 \pm 0.202) \times 10^{-2}$ \\
{$[200,290]$} & $(7.709 \pm 0.142 \pm 0.511) \times 10^{-4}$ & $(8.756 \pm 0.161 \pm 0.689) \times 10^{-3}$ \\
{$[290,400]$} & $(1.665 \pm 0.038 \pm 0.119) \times 10^{-4}$ & $(1.891 \pm 0.044 \pm 0.173) \times 10^{-3}$ \\
{$[400,550]$} & $(2.593 \pm 0.162 \pm 0.43) \times 10^{-5}$ & $(2.945 \pm 0.184 \pm 0.523) \times 10^{-4}$ \\
\hline
\end{tabular}

Table 18. The measured differential cross section and bin boundaries for each bin of the normalized and absolute measurements of the t $\bar{t}$ differential cross section at particle level in the fiducial phase space as a function of $p_{\mathrm{T}}^{\mathrm{t}}$ (trailing) are tabulated.

\begin{tabular}{|c|c|c|}
\hline$p_{\mathrm{T}}^{\mathrm{t}}(\mathrm{t} \overline{\mathrm{t}} \mathrm{RF})[\mathrm{GeV}]$ & $\frac{1}{\sigma} \frac{\mathrm{d} \sigma}{\mathrm{d} p_{\mathrm{T}}^{\mathrm{t}}(\mathrm{t} \overline{\mathrm{t}} \mathrm{RF})}\left[\mathrm{GeV}^{-1}\right]$ & $\frac{\mathrm{d} \sigma}{\mathrm{d} p_{\mathrm{T}}^{\mathrm{t}}(\mathrm{t} \overline{\mathrm{t}} \mathrm{RF})}[\mathrm{pb} / \mathrm{GeV}]$ \\
\hline$[0,65]$ & $(4.38 \pm 0.037 \pm 0.174) \times 10^{-3}$ & $(4.973 \pm 0.044 \pm 0.355) \times 10^{-2}$ \\
\hline$[65,125]$ & $(5.94 \pm 0.056 \pm 0.217) \times 10^{-3}$ & $(6.744 \pm 0.065 \pm 0.538) \times 10^{-2}$ \\
\hline$[125,200]$ & $(3.207 \pm 0.031 \pm 0.097) \times 10^{-3}$ & $(3.641 \pm 0.037 \pm 0.236) \times 10^{-2}$ \\
\hline$[200,290]$ & $(9.766 \pm 0.148 \pm 0.417) \times 10^{-4}$ & $(1.109 \pm 0.017 \pm 0.069) \times 10^{-2}$ \\
\hline$[290,400]$ & $(2.273 \pm 0.042 \pm 0.108) \times 10^{-4}$ & $(2.58 \pm 0.048 \pm 0.178) \times 10^{-3}$ \\
\hline$[400,550]$ & $(3.628 \pm 0.181 \pm 0.385) \times 10^{-5}$ & $(4.119 \pm 0.205 \pm 0.515) \times 10^{-4}$ \\
\hline
\end{tabular}

Table 19. The measured differential cross section and bin boundaries for each bin of the normalized and absolute measurements of the $t \bar{t}$ differential cross section at particle level in the fiducial phase space as a function of $p_{\mathrm{T}}^{\mathrm{t}}(\mathrm{t} \overline{\mathrm{t}} \mathrm{RF})$ are tabulated. 


\begin{tabular}{|ccc|}
\hline$y_{\mathrm{t}}$ & $\frac{1}{\sigma} \frac{\mathrm{d} \sigma}{\mathrm{d} y_{\mathrm{t}}}$ & $\frac{\mathrm{d} \sigma}{\mathrm{d} y_{\mathrm{t}}}[\mathrm{pb}]$ \\
\hline$[-2.6,-1.8]$ & $(3.154 \pm 0.061 \pm 0.171) \times 10^{-2}$ & $0.356 \pm 0.007 \pm 0.034$ \\
{$[-1.8,-1.35]$} & $0.145 \pm 0.001 \pm 0.004$ & $1.643 \pm 0.016 \pm 0.11$ \\
{$[-1.35,-0.9]$} & $0.248 \pm 0.002 \pm 0.005$ & $2.806 \pm 0.027 \pm 0.176$ \\
{$[-0.9,-0.45]$} & $0.314 \pm 0.003 \pm 0.006$ & $3.548 \pm 0.032 \pm 0.228$ \\
{$[-0.45,0]$} & $0.343 \pm 0.003 \pm 0.01$ & $3.878 \pm 0.034 \pm 0.248$ \\
{$[0,0.45]$} & $0.346 \pm 0.003 \pm 0.011$ & $3.913 \pm 0.034 \pm 0.273$ \\
{$[0.45,0.9]$} & $0.32 \pm 0.003 \pm 0.01$ & $3.622 \pm 0.031 \pm 0.235$ \\
{$[0.9,1.35]$} & $0.244 \pm 0.002 \pm 0.005$ & $2.753 \pm 0.025 \pm 0.178$ \\
{$[1.35,1.8]$} & $0.146 \pm 0.001 \pm 0.005$ & $1.655 \pm 0.016 \pm 0.12$ \\
{$[1.8,2.6]$} & $(3.31 \pm 0.06 \pm 0.144) \times 10^{-2}$ & $0.374 \pm 0.007 \pm 0.031$ \\
\hline
\end{tabular}

Table 20. The measured differential cross section and bin boundaries for each bin of the normalized and absolute measurements of the t $\bar{t}$ differential cross section at particle level in the fiducial phase space as a function of $y_{\mathrm{t}}$ are tabulated.

\begin{tabular}{|ccc|}
\hline$y_{\overline{\mathrm{t}}}$ & $\frac{1}{\sigma} \frac{\mathrm{d} \sigma}{\mathrm{d} y_{\overline{\mathrm{t}}}}$ & $\frac{\mathrm{d} \sigma}{\mathrm{d} y_{\overline{\mathrm{t}}}}[\mathrm{pb}]$ \\
\hline$[-2.6,-1.8]$ & $(3.21 \pm 0.061 \pm 0.163) \times 10^{-2}$ & $0.363 \pm 0.007 \pm 0.033$ \\
{$[-1.8,-1.35]$} & $0.143 \pm 0.001 \pm 0.003$ & $1.619 \pm 0.016 \pm 0.115$ \\
{$[-1.35,-0.9]$} & $0.246 \pm 0.002 \pm 0.007$ & $2.785 \pm 0.027 \pm 0.182$ \\
{$[-0.9,-0.45]$} & $0.313 \pm 0.003 \pm 0.007$ & $3.537 \pm 0.032 \pm 0.222$ \\
{$[-0.45,0]$} & $0.35 \pm 0.003 \pm 0.007$ & $3.961 \pm 0.035 \pm 0.258$ \\
{$[0,0.45]$} & $0.349 \pm 0.003 \pm 0.009$ & $3.943 \pm 0.034 \pm 0.257$ \\
{$[0.45,0.9]$} & $0.312 \pm 0.003 \pm 0.006$ & $3.522 \pm 0.031 \pm 0.218$ \\
{$[0.9,1.35]$} & $0.252 \pm 0.002 \pm 0.009$ & $2.846 \pm 0.025 \pm 0.189$ \\
{$[1.35,1.8]$} & $0.143 \pm 0.001 \pm 0.008$ & $1.62 \pm 0.016 \pm 0.138$ \\
{$[1.8,2.6]$} & $(3.187 \pm 0.06 \pm 0.206) \times 10^{-2}$ & $0.36 \pm 0.007 \pm 0.034$ \\
\hline
\end{tabular}

Table 21. The measured differential cross section and bin boundaries for each bin of the normalized and absolute measurements of the $t \bar{t}$ differential cross section at particle level in the fiducial phase space as a function of $y_{\overline{\mathrm{t}}}$ are tabulated. 


\begin{tabular}{|ccc|}
\hline$y_{\mathrm{t}}$ (leading) & $\frac{1}{\sigma} \frac{\mathrm{d} \sigma}{\left.\mathrm{d} y_{\mathrm{t}} \text { (leading }\right)}$ & $\frac{\mathrm{d} \sigma}{\mathrm{d} y_{\mathrm{t}}(\text { leading })}[\mathrm{pb}]$ \\
\hline$[-2.6,-1.65]$ & $(4.328 \pm 0.075 \pm 0.229) \times 10^{-2}$ & $0.489 \pm 0.009 \pm 0.046$ \\
{$[-1.65,-1.1]$} & $0.19 \pm 0.002 \pm 0.005$ & $2.148 \pm 0.02 \pm 0.153$ \\
{$[-1.1,-0.55]$} & $0.299 \pm 0.002 \pm 0.006$ & $3.378 \pm 0.027 \pm 0.206$ \\
{$[-0.55,0]$} & $0.344 \pm 0.003 \pm 0.007$ & $3.891 \pm 0.031 \pm 0.237$ \\
{$[0,0.55]$} & $0.344 \pm 0.003 \pm 0.007$ & $3.89 \pm 0.032 \pm 0.237$ \\
{$[0.55,1.1]$} & $0.3 \pm 0.002 \pm 0.004$ & $3.389 \pm 0.028 \pm 0.206$ \\
{$[1.1,1.65]$} & $0.188 \pm 0.002 \pm 0.007$ & $2.126 \pm 0.02 \pm 0.167$ \\
{$[1.65,2.6]$} & $(4.542 \pm 0.071 \pm 0.274) \times 10^{-2}$ & $0.513 \pm 0.008 \pm 0.045$ \\
\hline
\end{tabular}

Table 22. The measured differential cross section and bin boundaries for each bin of the normalized

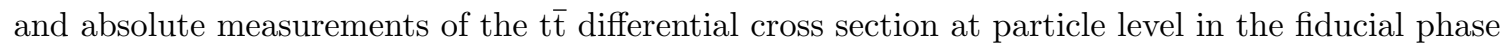
space as a function of $y_{\mathrm{t}}$ (leading) are tabulated.

\begin{tabular}{|ccc|}
\hline$y_{\mathrm{t}}$ (trailing) & $\frac{1}{\sigma} \frac{\mathrm{d} \sigma}{\mathrm{d} y_{\mathrm{t}} \text { (trailing) }}$ & $\frac{\mathrm{d} \sigma}{\left.\mathrm{d} y_{\mathrm{t}} \text { (trailing }\right)}[\mathrm{pb}]$ \\
\hline$[-2.6,-1.65]$ & $(4.461 \pm 0.08 \pm 0.233) \times 10^{-2}$ & $0.504 \pm 0.009 \pm 0.048$ \\
{$[-1.65,-1.1]$} & $0.19 \pm 0.002 \pm 0.006$ & $2.151 \pm 0.021 \pm 0.15$ \\
{$[-1.1,-0.55]$} & $0.296 \pm 0.002 \pm 0.009$ & $3.351 \pm 0.029 \pm 0.209$ \\
{$[-0.55,0]$} & $0.342 \pm 0.003 \pm 0.007$ & $3.86 \pm 0.033 \pm 0.253$ \\
{$[0,0.55]$} & $0.34 \pm 0.003 \pm 0.013$ & $3.845 \pm 0.034 \pm 0.278$ \\
{$[0.55,1.1]$} & $0.301 \pm 0.003 \pm 0.008$ & $3.403 \pm 0.03 \pm 0.213$ \\
{$[1.1,1.65]$} & $0.195 \pm 0.002 \pm 0.005$ & $2.203 \pm 0.021 \pm 0.148$ \\
{$[1.65,2.6]$} & $(4.423 \pm 0.076 \pm 0.201) \times 10^{-2}$ & $0.5 \pm 0.009 \pm 0.041$ \\
\hline
\end{tabular}

Table 23. The measured differential cross section and bin boundaries for each bin of the normalized

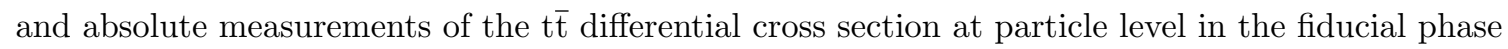
space as a function of $y_{\mathrm{t}}$ (trailing) are tabulated. 


\begin{tabular}{|c|c|c|}
\hline$p_{\mathrm{T}}^{\mathrm{t} \overline{\mathrm{t}}}$ & $\frac{1}{\sigma} \frac{\mathrm{d} \sigma}{\mathrm{d} p_{\mathrm{T}}^{\mathrm{t \overline { \textrm {t } }}}}$ & $\frac{\mathrm{d} \sigma}{\mathrm{d} p_{\mathrm{T}}^{\mathrm{tt}}}[\mathrm{pb}]$ \\
\hline$[0,40]$ & $(1.171 \pm 0.006 \pm 0.078) \times 10^{-2}$ & $0.132 \pm 0.001 \pm 0.011$ \\
\hline$[40,100]$ & $(5.35 \pm 0.045 \pm 0.582) \times 10^{-3}$ & $(6.05 \pm 0.052 \pm 0.781) \times 10^{-2}$ \\
\hline$[100,200]$ & $(1.579 \pm 0.014 \pm 0.06) \times 10^{-3}$ & $(1.785 \pm 0.016 \pm 0.128) \times 10^{-2}$ \\
\hline$[200,310]$ & $(3.509 \pm 0.053 \pm 0.128) \times 10^{-4}$ & $(3.968 \pm 0.06 \pm 0.305) \times 10^{-3}$ \\
\hline$[310,420]$ & $(9.53 \pm 0.218 \pm 0.562) \times 10^{-5}$ & $(1.078 \pm 0.025 \pm 0.096) \times 10^{-3}$ \\
\hline$[420,570]$ & $(2.45 \pm 0.099 \pm 0.158) \times 10^{-5}$ & $(2.771 \pm 0.112 \pm 0.265) \times 10^{-4}$ \\
\hline
\end{tabular}

Table 24. The measured differential cross section and bin boundaries for each bin of the normalized and absolute measurements of the t $\bar{t}$ differential cross section at particle level in the fiducial phase space as a function of $p_{\mathrm{T}}^{\mathrm{t} \overline{\mathrm{t}}}$ are tabulated.

\begin{tabular}{|ccc|}
\hline$y_{\mathrm{t} \overline{\mathrm{t}}}$ & $\frac{1}{\sigma} \frac{\mathrm{d} \sigma}{\mathrm{d} y_{\mathrm{t} \overline{\mathrm{t}}}}$ & $\frac{\mathrm{d} \sigma}{\mathrm{d} y_{\mathrm{t} \overline{\mathrm{t}}}}[\mathrm{pb}]$ \\
\hline$[-2.6,-1.6]$ & $(1.413 \pm 0.035 \pm 0.165) \times 10^{-2}$ & $0.16 \pm 0.004 \pm 0.023$ \\
{$[-1.6,-1.2]$} & $0.128 \pm 0.001 \pm 0.003$ & $1.446 \pm 0.016 \pm 0.098$ \\
{$[-1.2,-0.8]$} & $0.26 \pm 0.002 \pm 0.008$ & $2.941 \pm 0.029 \pm 0.192$ \\
{$[-0.8,-0.4]$} & $0.373 \pm 0.003 \pm 0.009$ & $4.213 \pm 0.036 \pm 0.279$ \\
{$[-0.4,0]$} & $0.458 \pm 0.004 \pm 0.009$ & $5.175 \pm 0.041 \pm 0.299$ \\
{$[0,0.4]$} & $0.44 \pm 0.003 \pm 0.009$ & $4.971 \pm 0.04 \pm 0.331$ \\
{$[0.4,0.8]$} & $0.378 \pm 0.003 \pm 0.007$ & $4.271 \pm 0.035 \pm 0.277$ \\
{$[0.8,1.2]$} & $0.265 \pm 0.002 \pm 0.007$ & $2.995 \pm 0.027 \pm 0.186$ \\
{$[1.2,1.6]$} & $0.125 \pm 0.001 \pm 0.004$ & $1.416 \pm 0.015 \pm 0.105$ \\
{$[1.6,2.6]$} & $(1.545 \pm 0.035 \pm 0.087) \times 10^{-2}$ & $0.175 \pm 0.004 \pm 0.017$ \\
\hline
\end{tabular}

Table 25. The measured differential cross section and bin boundaries for each bin of the normalized and absolute measurements of the $t \bar{t}$ differential cross section at particle level in the fiducial phase space as a function of $y_{\mathrm{t} \overline{\mathrm{t}}}$ are tabulated. 


\begin{tabular}{|ccc|}
\hline$m_{\mathrm{t} \overline{\mathrm{t}}}[\mathrm{GeV}]$ & $\frac{1}{\sigma} \frac{\mathrm{d} \sigma}{\mathrm{d} m_{\mathrm{t} \overline{\mathrm{t}}}}\left[\mathrm{GeV}^{-1}\right]$ & $\frac{\mathrm{d} \sigma}{\mathrm{d} m_{\mathrm{t} \overline{\mathrm{t}}}}[\mathrm{pb} / \mathrm{GeV}]$ \\
\hline$[300,380]$ & $(2.719 \pm 0.032 \pm 0.161) \times 10^{-3}$ & $(3.077 \pm 0.038 \pm 0.228) \times 10^{-2}$ \\
{$[380,470]$} & $(3.639 \pm 0.047 \pm 0.193) \times 10^{-3}$ & $(4.118 \pm 0.054 \pm 0.392) \times 10^{-2}$ \\
{$[470,620]$} & $(1.924 \pm 0.023 \pm 0.056) \times 10^{-3}$ & $(2.178 \pm 0.026 \pm 0.137) \times 10^{-2}$ \\
{$[620,820]$} & $(5.977 \pm 0.103 \pm 0.3) \times 10^{-4}$ & $(6.764 \pm 0.117 \pm 0.47) \times 10^{-3}$ \\
{$[820,1100]$} & $(1.305 \pm 0.036 \pm 0.097) \times 10^{-4}$ & $(1.477 \pm 0.041 \pm 0.139) \times 10^{-3}$ \\
{$[1100,1500]$} & $(2.19 \pm 0.081 \pm 0.176) \times 10^{-5}$ & $(2.478 \pm 0.091 \pm 0.245) \times 10^{-4}$ \\
{$[1500,2500]$} & $(1.526 \pm 0.142 \pm 0.33) \times 10^{-6}$ & $(1.727 \pm 0.16 \pm 0.376) \times 10^{-5}$ \\
\hline
\end{tabular}

Table 26. The measured differential cross section and bin boundaries for each bin of the normalized and absolute measurements of the t $\bar{t}$ differential cross section at particle level in the fiducial phase space as a function of $m_{\mathrm{t} \overline{\mathrm{t}}}$ are tabulated.

\begin{tabular}{|ccc|}
\hline$\Delta|y|(\mathrm{t}, \overline{\mathrm{t}})$ & $\frac{1}{\sigma} \frac{\mathrm{d} \sigma}{\mathrm{d} \Delta|y|(\mathrm{t}, \overline{\mathrm{t}})}$ & $\frac{\mathrm{d} \sigma}{\mathrm{d} \Delta|y|(\mathrm{t}, \overline{\mathrm{t}})}[\mathrm{pb}]$ \\
\hline$[-2.6,-1.4]$ & $(2.821 \pm 0.054 \pm 0.148) \times 10^{-2}$ & $0.319 \pm 0.006 \pm 0.027$ \\
{$[-1.4,-0.9]$} & $0.179 \pm 0.002 \pm 0.007$ & $2.021 \pm 0.022 \pm 0.153$ \\
{$[-0.9,-0.4]$} & $0.345 \pm 0.003 \pm 0.008$ & $3.903 \pm 0.036 \pm 0.271$ \\
{$[-0.4,0]$} & $0.5 \pm 0.004 \pm 0.014$ & $5.655 \pm 0.052 \pm 0.355$ \\
{$[0,0.4]$} & $0.507 \pm 0.005 \pm 0.011$ & $5.731 \pm 0.054 \pm 0.347$ \\
{$[0.4,0.9]$} & $0.349 \pm 0.003 \pm 0.011$ & $3.949 \pm 0.037 \pm 0.282$ \\
{$[0.9,1.4]$} & $0.183 \pm 0.002 \pm 0.005$ & $2.072 \pm 0.022 \pm 0.148$ \\
{$[1.4,2.6]$} & $(2.931 \pm 0.052 \pm 0.209) \times 10^{-2}$ & $0.331 \pm 0.006 \pm 0.03$ \\
\hline
\end{tabular}

Table 27. The measured differential cross section and bin boundaries for each bin of the normalized and absolute measurements of the $t \bar{t}$ differential cross section at particle level in the fiducial phase space as a function of $\Delta|y|(\mathrm{t}, \overline{\mathrm{t}})$ are tabulated.

\begin{tabular}{|ccc|}
\hline$\Delta \phi(\mathrm{t}, \overline{\mathrm{t}})[\mathrm{GeV}]$ & $\frac{1}{\sigma} \frac{\mathrm{d} \sigma}{\mathrm{d} \Delta \phi(\mathrm{t}, \overline{\mathrm{t}})}\left[\mathrm{GeV}^{-1}\right]$ & $\frac{\mathrm{d} \sigma}{\mathrm{d} \Delta \phi(\mathrm{t}, \overline{\mathrm{t}})}[\mathrm{pb} / \mathrm{GeV}]$ \\
\hline$[0,1.57]$ & $(6.284 \pm 0.067 \pm 0.309) \times 10^{-2}$ & $0.711 \pm 0.008 \pm 0.059$ \\
{$[1.57,2.67]$} & $0.223 \pm 0.001 \pm 0.008$ & $2.525 \pm 0.014 \pm 0.2$ \\
{$[2.67,3.02]$} & $1.051 \pm 0.005 \pm 0.025$ & $(1.189 \pm 0.007 \pm 0.081) \times 10$ \\
{$[3.02,3.142]$} & $2.362 \pm 0.017 \pm 0.134$ & $(2.672 \pm 0.02 \pm 0.194) \times 10$ \\
\hline
\end{tabular}

Table 28. The measured differential cross section and bin boundaries for each bin of the normalized

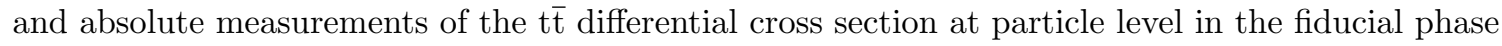
space as a function of $\Delta \phi(\mathrm{t}, \overline{\mathrm{t}})$ are tabulated. 


\begin{tabular}{|ccc|}
\hline$p_{\mathrm{T}}^{\ell}[\mathrm{GeV}]$ & $\frac{1}{\sigma} \frac{\mathrm{d} \sigma}{\mathrm{d} p_{\mathrm{T}}^{\ell}}\left[\mathrm{GeV}^{-1}\right]$ & $\frac{\mathrm{d} \sigma}{\mathrm{d} p_{\mathrm{T}}^{\ell}}[\mathrm{pb} / \mathrm{GeV}]$ \\
\hline$[20,40]$ & $(1.848 \pm 0.006 \pm 0.02) \times 10^{-2}$ & $0.209 \pm 0.001 \pm 0.014$ \\
{$[40,70]$} & $(1.251 \pm 0.004 \pm 0.011) \times 10^{-2}$ & $0.142 \pm 0.001 \pm 0.008$ \\
{$[70,120]$} & $(4.011 \pm 0.017 \pm 0.044) \times 10^{-3}$ & $(4.548 \pm 0.022 \pm 0.275) \times 10^{-2}$ \\
{$[120,180]$} & $(7.254 \pm 0.068 \pm 0.126) \times 10^{-4}$ & $(8.224 \pm 0.079 \pm 0.527) \times 10^{-3}$ \\
{$[180,400]$} & $(5.06 \pm 0.102 \pm 0.178) \times 10^{-5}$ & $(5.737 \pm 0.117 \pm 0.417) \times 10^{-4}$ \\
\hline
\end{tabular}

Table 29. The measured differential cross section and bin boundaries for each bin of the normalized

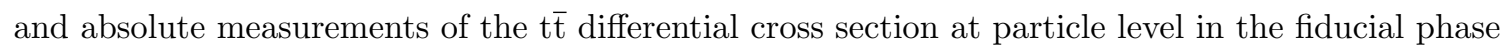
space as a function of $p_{\mathrm{T}}^{\ell}$ are tabulated.

\begin{tabular}{|ccc|}
\hline$p_{\mathrm{T}}^{\bar{\ell}}[\mathrm{GeV}]$ & $\frac{1}{\sigma} \frac{\mathrm{d} \sigma}{\mathrm{d} p_{\mathrm{T}}^{\ell}}\left[\mathrm{GeV}^{-1}\right]$ & $\frac{\mathrm{d} \sigma}{\mathrm{d} p_{\mathrm{T}}^{\ell}}[\mathrm{pb} / \mathrm{GeV}]$ \\
\hline$[20,40]$ & $(1.838 \pm 0.006 \pm 0.019) \times 10^{-2}$ & $0.208 \pm 0.001 \pm 0.014$ \\
{$[40,70]$} & $(1.26 \pm 0.004 \pm 0.006) \times 10^{-2}$ & $0.143 \pm 0.001 \pm 0.008$ \\
{$[70,120]$} & $(3.999 \pm 0.017 \pm 0.044) \times 10^{-3}$ & $(4.534 \pm 0.022 \pm 0.271) \times 10^{-2}$ \\
{$[120,180]$} & $(7.292 \pm 0.068 \pm 0.14) \times 10^{-4}$ & $(8.266 \pm 0.079 \pm 0.55) \times 10^{-3}$ \\
{$[180,400]$} & $(4.895 \pm 0.101 \pm 0.197) \times 10^{-5}$ & $(5.549 \pm 0.116 \pm 0.441) \times 10^{-4}$ \\
\hline
\end{tabular}

Table 30. The measured differential cross section and bin boundaries for each bin of the normalized and absolute measurements of the t $\bar{t}$ differential cross section at particle level in the fiducial phase space as a function of $p_{\mathrm{T}}^{\bar{\ell}}$ are tabulated.

\begin{tabular}{|ccc|}
\hline$p_{\mathrm{T}}^{\ell}$ (leading) $[\mathrm{GeV}]$ & $\frac{1}{\sigma} \frac{\mathrm{d} \sigma}{\mathrm{d} p_{\mathrm{T}}^{\ell}(\text { leading })}\left[\mathrm{GeV}^{-1}\right]$ & $\frac{\mathrm{d} \sigma}{\mathrm{d} p_{\mathrm{T}}^{\ell}(\text { leading })}[\mathrm{pb} / \mathrm{GeV}]$ \\
\hline$[20,40]$ & $(7.01 \pm 0.042 \pm 0.177) \times 10^{-3}$ & $(7.959 \pm 0.051 \pm 0.601) \times 10^{-2}$ \\
{$[40,70]$} & $(1.441 \pm 0.004 \pm 0.009) \times 10^{-2}$ & $0.164 \pm 0.001 \pm 0.01$ \\
{$[70,120]$} & $(6.562 \pm 0.021 \pm 0.061) \times 10^{-3}$ & $(7.45 \pm 0.028 \pm 0.445) \times 10^{-2}$ \\
{$[120,180]$} & $(1.312 \pm 0.009 \pm 0.02) \times 10^{-3}$ & $(1.489 \pm 0.011 \pm 0.096) \times 10^{-2}$ \\
{$[180,400]$} & $(9.39 \pm 0.138 \pm 0.32) \times 10^{-5}$ & $(1.066 \pm 0.016 \pm 0.079) \times 10^{-3}$ \\
\hline
\end{tabular}

Table 31. The measured differential cross section and bin boundaries for each bin of the normalized and absolute measurements of the t $\bar{t}$ differential cross section at particle level in the fiducial phase space as a function of $p_{\mathrm{T}}^{\ell}$ (leading) are tabulated. 


\begin{tabular}{|ccc|}
\hline$p_{\mathrm{T}}^{\ell}$ (trailing) $[\mathrm{GeV}]$ & $\frac{1}{\sigma} \frac{\mathrm{d} \sigma}{\left.\mathrm{d} p_{\mathrm{T}}^{\ell} \text { (trailing }\right)}\left[\mathrm{GeV}^{-1}\right]$ & $\frac{\mathrm{d} \sigma}{\mathrm{d} p_{\mathrm{T}}^{\ell} \text { trailing }}[\mathrm{pb} / \mathrm{GeV}]$ \\
\hline$[20,35]$ & $(3.184 \pm 0.008 \pm 0.032) \times 10^{-2}$ & $0.361 \pm 0.001 \pm 0.024$ \\
{$[35,50]$} & $(1.916 \pm 0.007 \pm 0.02) \times 10^{-2}$ & $0.217 \pm 0.001 \pm 0.012$ \\
{$[50,90]$} & $(5.063 \pm 0.021 \pm 0.058) \times 10^{-3}$ & $(5.74 \pm 0.027 \pm 0.345) \times 10^{-2}$ \\
{$[90,140]$} & $(5.493 \pm 0.064 \pm 0.124) \times 10^{-4}$ & $(6.228 \pm 0.073 \pm 0.41) \times 10^{-3}$ \\
{$[140,400]$} & $(1.903 \pm 0.058 \pm 0.062) \times 10^{-5}$ & $(2.158 \pm 0.065 \pm 0.157) \times 10^{-4}$ \\
\hline
\end{tabular}

Table 32. The measured differential cross section and bin boundaries for each bin of the normalized and absolute measurements of the t $\bar{t}$ differential cross section at particle level in the fiducial phase space as a function of $p_{\mathrm{T}}^{\ell}$ (trailing) are tabulated. 


\begin{tabular}{|ccc|}
\hline$\eta_{\ell}$ & $\frac{1}{\sigma} \frac{\mathrm{d} \sigma}{\mathrm{d} \eta_{\ell}}$ & $\frac{\mathrm{d} \sigma}{\mathrm{d} \eta_{\ell}}[\mathrm{pb}]$ \\
\hline$[-2.4,-2.1]$ & $(6.907 \pm 0.105 \pm 0.27) \times 10^{-2}$ & $0.781 \pm 0.012 \pm 0.061$ \\
{$[-2.1,-1.8]$} & $0.101 \pm 0.001 \pm 0.003$ & $1.143 \pm 0.014 \pm 0.086$ \\
{$[-1.8,-1.5]$} & $0.147 \pm 0.002 \pm 0.003$ & $1.665 \pm 0.017 \pm 0.103$ \\
{$[-1.5,-1.2]$} & $0.193 \pm 0.002 \pm 0.003$ & $2.186 \pm 0.02 \pm 0.141$ \\
{$[-1.2,-0.9]$} & $0.244 \pm 0.002 \pm 0.003$ & $2.755 \pm 0.022 \pm 0.171$ \\
{$[-0.9,-0.6]$} & $0.283 \pm 0.002 \pm 0.004$ & $3.203 \pm 0.023 \pm 0.193$ \\
{$[-0.6,-0.3]$} & $0.312 \pm 0.002 \pm 0.004$ & $3.524 \pm 0.024 \pm 0.215$ \\
{$[-0.3,0]$} & $0.318 \pm 0.002 \pm 0.003$ & $3.598 \pm 0.025 \pm 0.224$ \\
{$[0,0.3]$} & $0.317 \pm 0.002 \pm 0.003$ & $3.58 \pm 0.026 \pm 0.224$ \\
{$[0.3,0.6]$} & $0.311 \pm 0.002 \pm 0.003$ & $3.518 \pm 0.025 \pm 0.218$ \\
{$[0.6,0.9]$} & $0.28 \pm 0.002 \pm 0.002$ & $3.167 \pm 0.023 \pm 0.192$ \\
{$[0.9,1.2]$} & $0.246 \pm 0.002 \pm 0.004$ & $2.777 \pm 0.022 \pm 0.171$ \\
{$[1.2,1.5]$} & $0.191 \pm 0.002 \pm 0.003$ & $2.163 \pm 0.02 \pm 0.142$ \\
{$[1.5,1.8]$} & $0.151 \pm 0.002 \pm 0.003$ & $1.709 \pm 0.019 \pm 0.107$ \\
{$[1.8,2.1]$} & $0.104 \pm 0.001 \pm 0.003$ & $1.18 \pm 0.015 \pm 0.084$ \\
{$[2.1,2.4]$} & $(6.531 \pm 0.103 \pm 0.226) \times 10^{-2}$ & $0.738 \pm 0.012 \pm 0.055$ \\
\hline
\end{tabular}

Table 33. The measured differential cross section and bin boundaries for each bin of the normalized and absolute measurements of the t $\bar{t}$ differential cross section at particle level in the fiducial phase space as a function of $\eta_{\ell}$ are tabulated. 


\begin{tabular}{|ccc|}
\hline$\eta_{\bar{\ell}}$ & $\frac{1}{\sigma} \frac{\mathrm{d} \sigma}{\mathrm{d} \eta_{\bar{\ell}}}$ & $\frac{\mathrm{d} \sigma}{\mathrm{d} \eta_{\bar{\ell}}}[\mathrm{pb}]$ \\
\hline$[-2.4,-2.1]$ & $(6.86 \pm 0.106 \pm 0.191) \times 10^{-2}$ & $0.775 \pm 0.012 \pm 0.055$ \\
{$[-2.1,-1.8]$} & $0.104 \pm 0.001 \pm 0.003$ & $1.176 \pm 0.014 \pm 0.081$ \\
{$[-1.8,-1.5]$} & $0.151 \pm 0.002 \pm 0.003$ & $1.704 \pm 0.018 \pm 0.109$ \\
{$[-1.5,-1.2]$} & $0.198 \pm 0.002 \pm 0.002$ & $2.235 \pm 0.02 \pm 0.136$ \\
{$[-1.2,-0.9]$} & $0.244 \pm 0.002 \pm 0.003$ & $2.758 \pm 0.022 \pm 0.172$ \\
{$[-0.9,-0.6]$} & $0.28 \pm 0.002 \pm 0.003$ & $3.162 \pm 0.023 \pm 0.198$ \\
{$[-0.6,-0.3]$} & $0.306 \pm 0.002 \pm 0.002$ & $3.454 \pm 0.024 \pm 0.212$ \\
{$[-0.3,0]$} & $0.313 \pm 0.002 \pm 0.004$ & $3.533 \pm 0.025 \pm 0.225$ \\
{$[0,0.3]$} & $0.32 \pm 0.002 \pm 0.003$ & $3.612 \pm 0.026 \pm 0.225$ \\
{$[0.3,0.6]$} & $0.306 \pm 0.002 \pm 0.003$ & $3.459 \pm 0.025 \pm 0.208$ \\
{$[0.6,0.9]$} & $0.287 \pm 0.002 \pm 0.004$ & $3.239 \pm 0.023 \pm 0.199$ \\
{$[0.9,1.2]$} & $0.239 \pm 0.002 \pm 0.003$ & $2.702 \pm 0.022 \pm 0.176$ \\
{$[1.2,1.5]$} & $0.195 \pm 0.002 \pm 0.003$ & $2.205 \pm 0.02 \pm 0.139$ \\
{$[1.5,1.8]$} & $0.151 \pm 0.002 \pm 0.002$ & $1.703 \pm 0.018 \pm 0.109$ \\
{$[1.8,2.1]$} & $0.107 \pm 0.001 \pm 0.002$ & $1.21 \pm 0.015 \pm 0.08$ \\
{$[2.1,2.4]$} & $(6.635 \pm 0.104 \pm 0.203) \times 10^{-2}$ & $0.75 \pm 0.012 \pm 0.055$ \\
\hline
\end{tabular}

Table 34. The measured differential cross section and bin boundaries for each bin of the normalized and absolute measurements of the t $\bar{t}$ differential cross section at particle level in the fiducial phase space as a function of $\eta_{\bar{\ell}}$ are tabulated. 


\begin{tabular}{|ccc|}
\hline$\eta_{\ell}($ leading $)$ & $\frac{1}{\sigma} \frac{\mathrm{d} \sigma}{\left.\mathrm{d} \ell_{\ell} \text { leading }\right)}$ & $\frac{\mathrm{d} \sigma}{\left.\mathrm{d} \eta_{\ell} \text { leading }\right)}[\mathrm{pb}]$ \\
\hline$[-2.4,-2.1]$ & $(6.102 \pm 0.102 \pm 0.211) \times 10^{-2}$ & $0.69 \pm 0.012 \pm 0.052$ \\
{$[-2.1,-1.8]$} & $(9.44 \pm 0.124 \pm 0.304) \times 10^{-2}$ & $1.067 \pm 0.014 \pm 0.084$ \\
{$[-1.8,-1.5]$} & $0.146 \pm 0.002 \pm 0.002$ & $1.655 \pm 0.018 \pm 0.102$ \\
{$[-1.5,-1.2]$} & $0.194 \pm 0.002 \pm 0.003$ & $2.198 \pm 0.02 \pm 0.138$ \\
{$[-1.2,-0.9]$} & $0.243 \pm 0.002 \pm 0.003$ & $2.749 \pm 0.022 \pm 0.17$ \\
{$[-0.9,-0.6]$} & $0.29 \pm 0.002 \pm 0.003$ & $3.28 \pm 0.024 \pm 0.2$ \\
{$[-0.6,-0.3]$} & $0.318 \pm 0.002 \pm 0.004$ & $3.591 \pm 0.025 \pm 0.219$ \\
{$[-0.3,0]$} & $0.321 \pm 0.002 \pm 0.004$ & $3.625 \pm 0.025 \pm 0.23$ \\
{$[0,0.3]$} & $0.326 \pm 0.002 \pm 0.004$ & $3.689 \pm 0.026 \pm 0.23$ \\
{$[0.3,0.6]$} & $0.315 \pm 0.002 \pm 0.004$ & $3.563 \pm 0.026 \pm 0.219$ \\
{$[0.6,0.9]$} & $0.287 \pm 0.002 \pm 0.004$ & $3.247 \pm 0.024 \pm 0.197$ \\
{$[0.9,1.2]$} & $0.245 \pm 0.002 \pm 0.003$ & $2.767 \pm 0.023 \pm 0.175$ \\
{$[1.2,1.5]$} & $0.192 \pm 0.002 \pm 0.003$ & $2.168 \pm 0.021 \pm 0.139$ \\
{$[1.5,1.8]$} & $0.146 \pm 0.002 \pm 0.004$ & $1.652 \pm 0.019 \pm 0.106$ \\
{$[1.8,2.1]$} & $(9.55 \pm 0.129 \pm 0.268) \times 10^{-2}$ & $1.08 \pm 0.015 \pm 0.082$ \\
{$[2.1,2.4]$} & $(5.913 \pm 0.101 \pm 0.159) \times 10^{-2}$ & $0.669 \pm 0.012 \pm 0.049$ \\
\hline
\end{tabular}

Table 35. The measured differential cross section and bin boundaries for each bin of the normalized and absolute measurements of the $t \bar{t}$ differential cross section at particle level in the fiducial phase space as a function of $\eta_{\ell}$ (leading) are tabulated. 


\begin{tabular}{|ccc|}
\hline$\eta_{\ell}$ (trailing) & $\frac{1}{\sigma} \frac{\mathrm{d} \sigma}{\mathrm{d} \eta_{\ell} \text { (trailing) }}$ & $\frac{\mathrm{d} \sigma}{\left.\mathrm{d} \eta_{\ell} \text { trailing }\right)}[\mathrm{pb}]$ \\
\hline$[-2.4,-2.1]$ & $(7.656 \pm 0.113 \pm 0.167) \times 10^{-2}$ & $0.866 \pm 0.013 \pm 0.059$ \\
{$[-2.1,-1.8]$} & $0.111 \pm 0.001 \pm 0.003$ & $1.25 \pm 0.015 \pm 0.087$ \\
{$[-1.8,-1.5]$} & $0.152 \pm 0.002 \pm 0.003$ & $1.715 \pm 0.018 \pm 0.112$ \\
{$[-1.5,-1.2]$} & $0.197 \pm 0.002 \pm 0.003$ & $2.225 \pm 0.021 \pm 0.139$ \\
{$[-1.2,-0.9]$} & $0.245 \pm 0.002 \pm 0.004$ & $2.765 \pm 0.022 \pm 0.173$ \\
{$[-0.9,-0.6]$} & $0.273 \pm 0.002 \pm 0.004$ & $3.086 \pm 0.023 \pm 0.189$ \\
{$[-0.6,-0.3]$} & $0.3 \pm 0.002 \pm 0.002$ & $3.388 \pm 0.024 \pm 0.209$ \\
{$[-0.3,0]$} & $0.31 \pm 0.002 \pm 0.004$ & $3.504 \pm 0.025 \pm 0.218$ \\
{$[0,0.3]$} & $0.309 \pm 0.002 \pm 0.003$ & $3.498 \pm 0.026 \pm 0.22$ \\
{$[0.3,0.6]$} & $0.303 \pm 0.002 \pm 0.003$ & $3.422 \pm 0.025 \pm 0.208$ \\
{$[0.6,0.9]$} & $0.28 \pm 0.002 \pm 0.003$ & $3.166 \pm 0.024 \pm 0.195$ \\
{$[0.9,1.2]$} & $0.24 \pm 0.002 \pm 0.003$ & $2.713 \pm 0.023 \pm 0.172$ \\
{$[1.2,1.5]$} & $0.195 \pm 0.002 \pm 0.002$ & $2.2 \pm 0.021 \pm 0.141$ \\
{$[1.5,1.8]$} & $0.156 \pm 0.002 \pm 0.003$ & $1.762 \pm 0.019 \pm 0.116$ \\
{$[1.8,2.1]$} & $0.116 \pm 0.001 \pm 0.003$ & $1.31 \pm 0.016 \pm 0.086$ \\
{$[2.1,2.4]$} & $(7.226 \pm 0.112 \pm 0.232) \times 10^{-2}$ & $0.817 \pm 0.013 \pm 0.06$ \\
\hline
\end{tabular}

Table 36. The measured differential cross section and bin boundaries for each bin of the normalized and absolute measurements of the t $\bar{t}$ differential cross section at particle level in the fiducial phase space as a function of $\eta_{\ell}$ (trailing) are tabulated.

\begin{tabular}{|ccc|}
\hline$p_{\mathrm{T}}^{\ell \bar{\ell}}[\mathrm{GeV}]$ & $\frac{1}{\sigma} \frac{\mathrm{d} \sigma}{\mathrm{d} p_{\mathrm{T}}^{\ell \ell}}\left[\mathrm{GeV}^{-1}\right]$ & $\frac{\mathrm{d} \sigma}{\mathrm{d} p_{\mathrm{T}}^{\ell \bar{\ell}}}[\mathrm{pb} / \mathrm{GeV}]$ \\
\hline$[0,10]$ & $(1.691 \pm 0.032 \pm 0.068) \times 10^{-3}$ & $(1.913 \pm 0.036 \pm 0.155) \times 10^{-2}$ \\
{$[10,20]$} & $(4.682 \pm 0.049 \pm 0.109) \times 10^{-3}$ & $(5.297 \pm 0.057 \pm 0.371) \times 10^{-2}$ \\
{$[20,40]$} & $(7.593 \pm 0.042 \pm 0.076) \times 10^{-3}$ & $(8.591 \pm 0.052 \pm 0.562) \times 10^{-2}$ \\
{$[40,60]$} & $(1.094 \pm 0.005 \pm 0.009) \times 10^{-2}$ & $0.124 \pm 0.001 \pm 0.008$ \\
{$[60,100]$} & $(9.554 \pm 0.028 \pm 0.05) \times 10^{-3}$ & $0.108 \pm<10^{-3} \pm 0.006$ \\
{$[100,150]$} & $(3.04 \pm 0.016 \pm 0.031) \times 10^{-3}$ & $(3.44 \pm 0.019 \pm 0.21) \times 10^{-2}$ \\
{$[150,400]$} & $(1.259 \pm 0.015 \pm 0.043) \times 10^{-4}$ & $(1.425 \pm 0.017 \pm 0.108) \times 10^{-3}$ \\
\hline
\end{tabular}

Table 37. The measured differential cross section and bin boundaries for each bin of the normalized and absolute measurements of the $t \bar{t}$ differential cross section at particle level in the fiducial phase space as a function of $p_{\mathrm{T}}^{\ell \bar{\ell}}$ are tabulated. 


\begin{tabular}{|ccc|}
\hline$m_{\ell \bar{\ell}}[\mathrm{GeV}]$ & $\frac{1}{\sigma} \frac{\mathrm{d} \sigma}{\mathrm{d} m_{\ell \bar{\ell}}}\left[\mathrm{GeV}^{-1}\right]$ & $\frac{\mathrm{d} \sigma}{\mathrm{d} m_{\ell \bar{\ell}}}[\mathrm{pb} / \mathrm{GeV}]$ \\
\hline$[20,30]$ & $(3.44 \pm 0.042 \pm 0.14) \times 10^{-3}$ & $(3.903 \pm 0.048 \pm 0.337) \times 10^{-2}$ \\
{$[30,50]$} & $(5.338 \pm 0.033 \pm 0.101) \times 10^{-3}$ & $(6.058 \pm 0.041 \pm 0.419) \times 10^{-2}$ \\
{$[50,76]$} & $(7.656 \pm 0.034 \pm 0.144) \times 10^{-3}$ & $(8.688 \pm 0.042 \pm 0.608) \times 10^{-2}$ \\
{$[76,106]$} & $(7.475 \pm 0.037 \pm 0.123) \times 10^{-3}$ & $(8.482 \pm 0.049 \pm 0.482) \times 10^{-2}$ \\
{$[106,130]$} & $(5.446 \pm 0.031 \pm 0.042) \times 10^{-3}$ & $(6.18 \pm 0.038 \pm 0.372) \times 10^{-2}$ \\
{$[130,170]$} & $(3.481 \pm 0.019 \pm 0.041) \times 10^{-3}$ & $(3.95 \pm 0.022 \pm 0.233) \times 10^{-2}$ \\
{$[170,260]$} & $(1.342 \pm 0.007 \pm 0.02) \times 10^{-3}$ & $(1.523 \pm 0.009 \pm 0.091) \times 10^{-2}$ \\
{$[260,650]$} & $(1.149 \pm 0.01 \pm 0.021) \times 10^{-4}$ & $(1.304 \pm 0.012 \pm 0.081) \times 10^{-3}$ \\
\hline
\end{tabular}

Table 38. The measured differential cross section and bin boundaries for each bin of the normalized and absolute measurements of the $t \bar{t}$ differential cross section at particle level in the fiducial phase space as a function of $m_{\ell \bar{\ell}}$ are tabulated.

\begin{tabular}{|ccc|}
\hline$\Delta \phi(\ell, \bar{\ell})$ & $\frac{1}{\sigma} \frac{\mathrm{d} \sigma}{\mathrm{d} \Delta \phi(\ell, \bar{\ell})}$ & $\frac{\mathrm{d} \sigma}{\mathrm{d} \Delta \phi(\ell, \bar{\ell})}[\mathrm{pb}]$ \\
\hline$[0,0.4]$ & $0.194 \pm 0.001 \pm 0.003$ & $2.191 \pm 0.017 \pm 0.14$ \\
{$[0.4,0.78]$} & $0.247 \pm 0.002 \pm 0.003$ & $2.793 \pm 0.02 \pm 0.179$ \\
{$[0.78,1.14]$} & $0.278 \pm 0.002 \pm 0.003$ & $3.141 \pm 0.023 \pm 0.196$ \\
{$[1.14,1.48]$} & $0.299 \pm 0.002 \pm 0.003$ & $3.381 \pm 0.024 \pm 0.205$ \\
{$[1.48,1.8]$} & $0.326 \pm 0.002 \pm 0.002$ & $3.688 \pm 0.026 \pm 0.225$ \\
{$[1.8,2.1]$} & $0.352 \pm 0.002 \pm 0.003$ & $3.98 \pm 0.027 \pm 0.247$ \\
{$[2.1,2.38]$} & $0.374 \pm 0.002 \pm 0.003$ & $4.235 \pm 0.028 \pm 0.269$ \\
{$[2.38,2.64]$} & $0.393 \pm 0.002 \pm 0.003$ & $4.449 \pm 0.028 \pm 0.273$ \\
{$[2.64,2.89]$} & $0.42 \pm 0.002 \pm 0.004$ & $4.749 \pm 0.029 \pm 0.296$ \\
{$[2.89,3.142]$} & $0.418 \pm 0.003 \pm 0.004$ & $4.724 \pm 0.03 \pm 0.301$ \\
\hline
\end{tabular}

Table 39. The measured differential cross section and bin boundaries for each bin of the normalized and absolute measurements of the t $\overline{\mathrm{t}}$ differential cross section at particle level in the fiducial phase space as a function of $\Delta \phi(\ell, \bar{\ell})$ are tabulated. 


\begin{tabular}{|ccc|}
\hline$\Delta \eta(\ell, \bar{\ell})$ & $\frac{1}{\sigma} \frac{\mathrm{d} \sigma}{\mathrm{d} \Delta \eta(\ell, \bar{\ell})}$ & $\frac{\mathrm{d} \sigma}{\mathrm{d} \Delta \eta(\ell, \bar{\ell})}[\mathrm{pb}]$ \\
\hline$[-2.4,-1.7]$ & $(3.516 \pm 0.047 \pm 0.083) \times 10^{-2}$ & $0.398 \pm 0.005 \pm 0.025$ \\
{$[-1.7,-1.2]$} & $0.135 \pm 0.001 \pm 0.002$ & $1.526 \pm 0.012 \pm 0.091$ \\
{$[-1.2,-0.8]$} & $0.245 \pm 0.002 \pm 0.003$ & $2.766 \pm 0.02 \pm 0.171$ \\
{$[-0.8,-0.4]$} & $0.352 \pm 0.002 \pm 0.003$ & $3.98 \pm 0.024 \pm 0.248$ \\
{$[-0.4,0]$} & $0.43 \pm 0.002 \pm 0.004$ & $4.864 \pm 0.026 \pm 0.31$ \\
{$[0,0.4]$} & $0.428 \pm 0.002 \pm 0.003$ & $4.844 \pm 0.026 \pm 0.305$ \\
{$[0.4,0.8]$} & $0.349 \pm 0.002 \pm 0.003$ & $3.951 \pm 0.023 \pm 0.246$ \\
{$[0.8,1.2]$} & $0.24 \pm 0.002 \pm 0.003$ & $2.715 \pm 0.018 \pm 0.17$ \\
{$[1.2,1.7]$} & $0.132 \pm 0.001 \pm 0.002$ & $1.495 \pm 0.012 \pm 0.093$ \\
{$[1.7,2.4]$} & $(3.41 \pm 0.046 \pm 0.085) \times 10^{-2}$ & $0.386 \pm 0.005 \pm 0.025$ \\
\hline
\end{tabular}

Table 40. The measured differential cross section and bin boundaries for each bin of the normalized and absolute measurements of the t $\overline{\mathrm{t}}$ differential cross section at particle level in the fiducial phase space as a function of $\Delta \eta(\ell, \bar{\ell})$ are tabulated.

\begin{tabular}{|ccc|}
\hline$p_{\mathrm{T}}^{\mathrm{b}}$ (leading) $[\mathrm{GeV}]$ & $\frac{1}{\sigma} \frac{\mathrm{d} \sigma}{\mathrm{d} p_{\mathrm{T}}^{\mathrm{b}}(\text { leading })}\left[\mathrm{GeV}^{-1}\right]$ & $\frac{\mathrm{d} \sigma}{\mathrm{d} p_{\mathrm{T}}^{\mathrm{b}}(\text { leading })}[\mathrm{pb} / \mathrm{GeV}]$ \\
\hline$[30,60]$ & $(6.234 \pm 0.041 \pm 0.373) \times 10^{-3}$ & $(7.039 \pm 0.048 \pm 0.553) \times 10^{-2}$ \\
{$[60,95]$} & $(1.163 \pm 0.005 \pm 0.014) \times 10^{-2}$ & $0.131 \pm 0.001 \pm 0.008$ \\
{$[95,150]$} & $(5.411 \pm 0.025 \pm 0.123) \times 10^{-3}$ & $(6.109 \pm 0.031 \pm 0.433) \times 10^{-2}$ \\
{$[150,230]$} & $(1.134 \pm 0.009 \pm 0.042) \times 10^{-3}$ & $(1.28 \pm 0.01 \pm 0.105) \times 10^{-2}$ \\
{$[230,500]$} & $(6.525 \pm 0.128 \pm 0.337) \times 10^{-5}$ & $(7.367 \pm 0.146 \pm 0.671) \times 10^{-4}$ \\
\hline
\end{tabular}

Table 41. The measured differential cross section and bin boundaries for each bin of the normalized and absolute measurements of the te differential cross section at particle level in the fiducial phase space as a function of $p_{\mathrm{T}}^{\mathrm{b}}$ (leading) are tabulated.

\begin{tabular}{|ccc|}
\hline$p_{\mathrm{T}}^{\mathrm{b}}$ (trailing) $[\mathrm{GeV}]$ & $\frac{1}{\sigma} \frac{\mathrm{d} \sigma}{\mathrm{d} p_{\mathrm{T}}^{\mathrm{b}}(\text { trailing })}\left[\mathrm{GeV}^{-1}\right]$ & $\frac{\mathrm{d} \sigma}{\left.\mathrm{d} p_{\mathrm{T}}^{\mathrm{b}} \text { (trailing }\right)}[\mathrm{pb} / \mathrm{GeV}]$ \\
\hline$[30,45]$ & $(2.48 \pm 0.012 \pm 0.096) \times 10^{-2}$ & $0.28 \pm 0.001 \pm 0.019$ \\
{$[45,70]$} & $(1.535 \pm 0.008 \pm 0.033) \times 10^{-2}$ & $0.173 \pm 0.001 \pm 0.011$ \\
{$[70,110]$} & $(4.691 \pm 0.032 \pm 0.148) \times 10^{-3}$ & $(5.293 \pm 0.039 \pm 0.377) \times 10^{-2}$ \\
{$[110,170]$} & $(8.159 \pm 0.101 \pm 0.302) \times 10^{-4}$ & $(9.206 \pm 0.117 \pm 0.7) \times 10^{-3}$ \\
{$[170,500]$} & $(2.304 \pm 0.078 \pm 0.205) \times 10^{-5}$ & $(2.6 \pm 0.088 \pm 0.292) \times 10^{-4}$ \\
\hline
\end{tabular}

Table 42. The measured differential cross section and bin boundaries for each bin of the normalized and absolute measurements of the t $\bar{t}$ differential cross section at particle level in the fiducial phase space as a function of $p_{\mathrm{T}}^{\mathrm{b}}$ (trailing) are tabulated. 


\begin{tabular}{|ccc|}
\hline$\eta_{\mathrm{b}}$ (leading) & $\frac{1}{\sigma} \frac{\mathrm{d} \sigma}{\mathrm{d} \eta_{\mathrm{b}}(\text { leading })}$ & $\frac{\mathrm{d} \sigma}{\mathrm{d} \eta_{\mathrm{b}} \text { (leading) }}[\mathrm{pb}]$ \\
\hline$[-2.4,-1.8]$ & $(8.029 \pm 0.099 \pm 0.414) \times 10^{-2}$ & $0.908 \pm 0.011 \pm 0.087$ \\
{$[-1.8,-1.2]$} & $0.162 \pm 0.001 \pm 0.004$ & $1.829 \pm 0.015 \pm 0.131$ \\
{$[-1.2,-0.6]$} & $0.269 \pm 0.002 \pm 0.004$ & $3.046 \pm 0.018 \pm 0.178$ \\
{$[-0.6,0]$} & $0.319 \pm 0.002 \pm 0.005$ & $3.605 \pm 0.02 \pm 0.213$ \\
{$[0,0.6]$} & $0.326 \pm 0.002 \pm 0.005$ & $3.685 \pm 0.021 \pm 0.209$ \\
{$[0.6,1.2]$} & $0.261 \pm 0.002 \pm 0.003$ & $2.954 \pm 0.019 \pm 0.176$ \\
{$[1.2,1.8]$} & $0.168 \pm 0.001 \pm 0.003$ & $1.9 \pm 0.015 \pm 0.131$ \\
{$[1.8,2.4]$} & $(8.11 \pm 0.093 \pm 0.348) \times 10^{-2}$ & $0.917 \pm 0.011 \pm 0.082$ \\
\hline
\end{tabular}

Table 43. The measured differential cross section and bin boundaries for each bin of the normalized

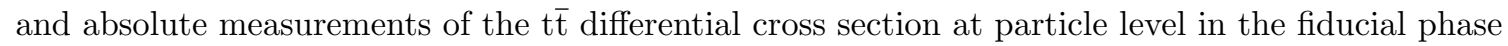
space as a function of $\eta_{\mathrm{b}}$ (leading) are tabulated.

\begin{tabular}{|ccc|}
\hline$\eta_{\mathrm{b}}$ (trailing) & $\frac{1}{\sigma} \frac{\mathrm{d} \sigma}{\mathrm{d} \eta_{\mathrm{b}}(\text { trailing })}$ & $\frac{\mathrm{d} \sigma}{\left.\mathrm{d} \eta_{\mathrm{b}} \text { (trailing }\right)}[\mathrm{pb}]$ \\
\hline$[-2.4,-1.8]$ & $(9.96 \pm 0.111 \pm 0.339) \times 10^{-2}$ & $1.126 \pm 0.013 \pm 0.087$ \\
{$[-1.8,-1.2]$} & $0.171 \pm 0.001 \pm 0.004$ & $1.937 \pm 0.016 \pm 0.136$ \\
{$[-1.2,-0.6]$} & $0.252 \pm 0.002 \pm 0.003$ & $2.847 \pm 0.019 \pm 0.173$ \\
{$[-0.6,0]$} & $0.301 \pm 0.002 \pm 0.005$ & $3.409 \pm 0.02 \pm 0.2$ \\
{$[0,0.6]$} & $0.31 \pm 0.002 \pm 0.005$ & $3.504 \pm 0.021 \pm 0.204$ \\
{$[0.6,1.2]$} & $0.258 \pm 0.002 \pm 0.003$ & $2.916 \pm 0.019 \pm 0.171$ \\
{$[1.2,1.8]$} & $0.178 \pm 0.001 \pm 0.003$ & $2.014 \pm 0.016 \pm 0.138$ \\
{$[1.8,2.4]$} & $(9.679 \pm 0.104 \pm 0.434) \times 10^{-2}$ & $1.095 \pm 0.012 \pm 0.098$ \\
\hline
\end{tabular}

Table 44. The measured differential cross section and bin boundaries for each bin of the normalized

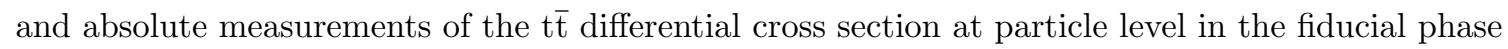
space as a function of $\eta_{\mathrm{b}}$ (trailing) are tabulated. 


\begin{tabular}{|ccc|}
\hline$p_{\mathrm{T}}^{\mathrm{b} \overline{\mathrm{b}}}[\mathrm{GeV}]$ & $\frac{1}{\sigma} \frac{\mathrm{d} \sigma}{\mathrm{d} p_{\mathrm{T}}^{\mathrm{bb}}}\left[\mathrm{GeV}^{-1}\right]$ & $\frac{\mathrm{d} \sigma}{\mathrm{d} p_{\mathrm{T}}^{\mathrm{bb}}}[\mathrm{pb} / \mathrm{GeV}]$ \\
\hline$[0,30]$ & $(3.518 \pm 0.029 \pm 0.144) \times 10^{-3}$ & $(3.976 \pm 0.034 \pm 0.26) \times 10^{-2}$ \\
{$[30,60]$} & $(7.185 \pm 0.038 \pm 0.216) \times 10^{-3}$ & $(8.12 \pm 0.047 \pm 0.469) \times 10^{-2}$ \\
{$[60,100]$} & $(8.916 \pm 0.034 \pm 0.16) \times 10^{-3}$ & $0.101 \pm<10^{-3} \pm 0.006$ \\
{$[100,180]$} & $(3.672 \pm 0.015 \pm 0.161) \times 10^{-3}$ & $(4.15 \pm 0.019 \pm 0.367) \times 10^{-2}$ \\
{$[180,400]$} & $(1.298 \pm 0.02 \pm 0.117) \times 10^{-4}$ & $(1.467 \pm 0.023 \pm 0.192) \times 10^{-3}$ \\
\hline
\end{tabular}

Table 45. The measured differential cross section and bin boundaries for each bin of the normalized and absolute measurements of the t $\bar{t}$ differential cross section at particle level in the fiducial phase space as a function of $p_{\mathrm{T}}^{\mathrm{b} \overline{\mathrm{b}}}$ are tabulated.

\begin{tabular}{|ccc|}
\hline$m_{\mathrm{b} \overline{\mathrm{b}}}[\mathrm{GeV}]$ & $\frac{1}{\sigma} \frac{\mathrm{d} \sigma}{\mathrm{d} m_{\mathrm{b \overline {b }}}}\left[\mathrm{GeV}^{-1}\right]$ & $\frac{\mathrm{d} \sigma}{\mathrm{d} m_{\mathrm{bb}}}[\mathrm{pb} / \mathrm{GeV}]$ \\
\hline$[0,60]$ & $(1.222 \pm 0.011 \pm 0.044) \times 10^{-3}$ & $(1.377 \pm 0.012 \pm 0.097) \times 10^{-2}$ \\
{$[60,120]$} & $(4.964 \pm 0.018 \pm 0.09) \times 10^{-3}$ & $(5.592 \pm 0.023 \pm 0.338) \times 10^{-2}$ \\
{$[120,240]$} & $(3.822 \pm 0.011 \pm 0.026) \times 10^{-3}$ & $(4.306 \pm 0.015 \pm 0.261) \times 10^{-2}$ \\
{$[240,650]$} & $(4.152 \pm 0.024 \pm 0.138) \times 10^{-4}$ & $(4.677 \pm 0.029 \pm 0.365) \times 10^{-3}$ \\
\hline
\end{tabular}

Table 46. The measured differential cross section and bin boundaries for each bin of the normalized and absolute measurements of the t $\bar{t}$ differential cross section at particle level in the fiducial phase space as a function of $m_{\mathrm{b} \overline{\mathrm{b}}}$ are tabulated.

\begin{tabular}{|ccc|}
\hline$N_{\text {jets }}$ & $\frac{1}{\sigma} \frac{\mathrm{d} \sigma}{\mathrm{d} N_{\text {jets }}}$ & $\frac{\mathrm{d} \sigma}{\mathrm{d} N_{\text {jets }}}[\mathrm{pb}]$ \\
\hline$[1.5,2.5]$ & $0.532 \pm 0.001 \pm 0.016$ & $6.01 \pm 0.02 \pm 0.341$ \\
{$[2.5,3.5]$} & $0.303 \pm 0.001 \pm 0.006$ & $3.42 \pm 0.017 \pm 0.24$ \\
{$[3.5,4.5]$} & $0.116 \pm 0.001 \pm 0.007$ & $1.307 \pm 0.012 \pm 0.128$ \\
{$[4.5,5.5]$} & $(3.68 \pm 0.06 \pm 0.313) \times 10^{-2}$ & $0.416 \pm 0.007 \pm 0.052$ \\
{$[5.5,6.5]$} & $(1.035 \pm 0.027 \pm 0.104) \times 10^{-2}$ & $0.117 \pm 0.003 \pm 0.016$ \\
{$[6.5,7.5]$} & $(2.514 \pm 0.137 \pm 0.426) \times 10^{-3}$ & $(2.84 \pm 0.155 \pm 0.571) \times 10^{-2}$ \\
\hline
\end{tabular}

Table 47. The measured differential cross section and bin boundaries for each bin of the normalized and absolute measurements of the t $\bar{t}$ differential cross section at particle level in the fiducial phase space as a function of $N_{\text {jets }}$ are tabulated. 


\section{Tables of $\chi^{2} /$ dof and $p$-values}

The $\chi^{2} /$ dof and $p$-values between data and all theoretical predictions for all measured differential cross sections are are tabulated in tables $48-55$. The $\chi^{2} /$ dof and $p$-value calculations take into account the inter-bin correlations of the data.

\begin{tabular}{|c|c|c|c|c|c|c|}
\hline & \multicolumn{2}{|c|}{ POWHEG+PYTHIA } & \multicolumn{2}{|c|}{ POWHEG + HERWIG ++} & \multicolumn{2}{|c|}{ MG5_aMC@NLO+PYTHIA $[\mathrm{FxFx}]$} \\
\hline & $\chi^{2} /$ dof & $p$-value & $\chi^{2} /$ dof & $p$-value & $\chi^{2} /$ dof & $p$-value \\
\hline$p_{\mathrm{T}}^{\mathrm{t}}$ & $43 / 5$ & $<10^{-3}$ & $6 / 5$ & 0.269 & $21 / 5$ & $<10^{-3}$ \\
\hline$p_{\mathrm{T}}^{\overline{\mathrm{t}}}$ & $35 / 5$ & $<10^{-3}$ & $7 / 5$ & 0.257 & $18 / 5$ & 0.003 \\
\hline$p_{\mathrm{T}}^{\mathrm{t}}$ (leading) & $42 / 5$ & $<10^{-3}$ & $3 / 5$ & 0.650 & $25 / 5$ & $<10^{-3}$ \\
\hline$p_{\mathrm{T}}^{\mathrm{t}}$ (trailing) & $44 / 5$ & $<10^{-3}$ & $14 / 5$ & 0.016 & $18 / 5$ & 0.003 \\
\hline$p_{\mathrm{T}}^{\mathrm{t}}(\mathrm{t} \overline{\mathrm{t}} \mathrm{RF})$ & $32 / 5$ & $<10^{-3}$ & $7 / 5$ & 0.209 & $16 / 5$ & 0.008 \\
\hline$y_{\mathrm{t}}$ & $6 / 9$ & 0.723 & $6 / 9$ & 0.758 & $5 / 9$ & 0.825 \\
\hline$y_{\overline{\mathrm{t}}}$ & $3 / 9$ & 0.976 & $3 / 9$ & 0.974 & $3 / 9$ & 0.966 \\
\hline$y_{\mathrm{t}}$ (leading) & $3 / 7$ & 0.862 & $3 / 7$ & 0.899 & $4 / 7$ & 0.820 \\
\hline$y_{\mathrm{t}}$ (trailing) & $3 / 7$ & 0.897 & $4 / 7$ & 0.795 & $2 / 7$ & 0.965 \\
\hline$p_{\mathrm{T}}^{\mathrm{t} \mathrm{t}}$ & $24 / 5$ & $<10^{-3}$ & $83 / 5$ & $<10^{-3}$ & $16 / 5$ & 0.007 \\
\hline$y_{\mathrm{t} \overline{\mathrm{t}}}$ & $3 / 9$ & 0.951 & $4 / 9$ & 0.916 & $4 / 9$ & 0.940 \\
\hline$m_{\mathrm{t} \overline{\mathrm{t}}}$ & $17 / 6$ & 0.009 & $2 / 6$ & 0.882 & $6 / 6$ & 0.382 \\
\hline$\Delta|y|(\mathrm{t}, \overline{\mathrm{t}})$ & $1 / 7$ & 0.987 & $3 / 7$ & 0.899 & $4 / 7$ & 0.738 \\
\hline$\Delta \phi(\mathrm{t}, \overline{\mathrm{t}})$ & $0 / 3$ & 0.978 & $2 / 3$ & 0.503 & $2 / 3$ & 0.633 \\
\hline
\end{tabular}

Table 48. The $\chi^{2} /$ dof and $p$-values quantifying the agreement between the three MC predictions and the measured, parton-level normalised cross sections are shown. 


\begin{tabular}{|c|c|c|c|c|c|c|c|c|c|c|}
\hline & \multicolumn{2}{|c|}{$\begin{array}{l}\mathrm{NNLO}+\alpha_{\mathrm{EW}}^{3} \\
(\mathrm{LUXQED} 17)\end{array}$} & \multicolumn{2}{|c|}{$\begin{array}{l}\mathrm{NNLO}+\alpha_{\mathrm{EW}}^{3} \\
(\mathrm{LUXQED} 17)\end{array}$} & \multicolumn{2}{|c|}{$\begin{array}{l}\mathrm{NNLO}+\alpha_{\mathrm{EW}}^{3} \\
(\mathrm{NNPDF} 3.1)\end{array}$} & \multicolumn{2}{|c|}{$\begin{array}{c}\text { NNLO+NNLL' } \\
(\text { NNPDF3.1) }\end{array}$} & \multicolumn{2}{|c|}{$\begin{array}{c}\text { NNLO+NNLL' } \\
\text { (NNPDF3.1) }\end{array}$} \\
\hline & $m_{\mathrm{t}}=$ & $3 \mathrm{GeV}$ & $m_{\mathrm{t}}=$ & $72.5 \mathrm{GeV}$ & $m_{\mathrm{t}}=$ & $\mathrm{GeV}$ & $m_{\mathrm{t}}$ & 3 & $m_{\mathrm{t}}=$ & $.5 \mathrm{Ge}$ \\
\hline & $\chi^{2} /$ dof & $p$-value & $\chi^{2} /$ dof & $p$-value & $\chi^{2} /$ dof & $p$-value & $\chi^{2} /$ dof & $p$-value & $\chi^{2} /$ dof & $p$-value \\
\hline$p_{\mathrm{T}}^{\mathrm{t}}$ & $16 / 5$ & 0.006 & $12 / 5$ & 036 & $12 / 5$ & .029 & $20 / 5$ & 0.001 & $15 / 5$ & 0.011 \\
\hline$p_{\mathrm{T}}^{\mathrm{t}}$ & $16 / 5$ & 0.007 & $12 / 5$ & 0.041 & $13 / 5$ & 0.027 & $17 / 5$ & 0.005 & $13 / 5$ & 0.026 \\
\hline$y_{\mathrm{t}}$ & $9 / 9$ & 0.434 & $8 /$ & 0.55 & $7 / 9$ & 0.665 & - & - & - & - \\
\hline$y_{\overline{\mathrm{t}}}$ & $4 / 9$ & 0.915 & $5 / 9$ & 0.875 & $2 / 9$ & 0.990 & - & - & - & - \\
\hline$p_{\mathrm{T}}^{\mathrm{t} \overline{\mathrm{t}}}$ & $26 / 5$ & $<10^{-3}$ & $25 / 5$ & $<10^{-3}$ & $25 / 5$ & $<10^{-}$ & - & - & - & - \\
\hline$y_{\mathrm{t} \overline{\mathrm{t}}}$ & $7 / 9$ & 0.597 & $7 / 9$ & 0.644 & $3 / 9$ & 0.960 & - & - & - & - \\
\hline$m_{\mathrm{t} \overline{\mathrm{t}}}$ & $34 / 6$ & $<10^{-3}$ & $24 / 6$ & $<10^{-3}$ & $28 / 6$ & $<10^{-3}$ & $30 / 6$ & $<10^{-3}$ & $19 / 6$ & 0.004 \\
\hline$\Delta|y|(\mathrm{t}, \overline{\mathrm{t}})$ & $5 / 7$ & 0.608 & $6 / 7$ & 0.537 & $2 / 7$ & 0.966 & - & - & - & - \\
\hline
\end{tabular}

Table 49. The $\chi^{2} /$ dof and $p$-values quantifying the agreement between the five theoretical predictions with $\mathrm{NNLO}+\alpha_{\mathrm{EW}}^{3}$ and NNLO+NNLL' precision and the measured, parton-level normalised cross sections are shown.

\begin{tabular}{|ccccc|}
\hline & $\mathrm{aN}^{3} \mathrm{LO}$ & aNNLO \\
& $(\mathrm{NNPDF} 3.0)$ & $(\mathrm{CT} 14 \mathrm{NNLO})$ \\
& $m_{\mathrm{t}}=172.5 \mathrm{GeV}$ & $m_{\mathrm{t}}=172.5 \mathrm{GeV}$ \\
& $\chi^{2} /$ dof & $p$-value & $\chi^{2} /$ dof & $p$-value \\
\hline$p_{\mathrm{T}}^{\mathrm{t}}$ & $7 / 5$ & 0.242 & $51 / 5$ & $<10^{-3}$ \\
$y_{\mathrm{t}}$ & $6 / 9$ & 0.698 & $60 / 9$ & $<10^{-3}$ \\
\hline
\end{tabular}

Table 50. The $\chi^{2} /$ dof and $p$-values quantifying the agreement between the two theoretical predictions with $\mathrm{aN}^{3} \mathrm{LO}$ and aNNLO precision and the measured, parton-level normalised cross sections are shown. 


\begin{tabular}{|c|c|c|c|c|c|c|}
\hline & \multicolumn{2}{|c|}{ POWHEG+PYTHIA } & \multicolumn{2}{|c|}{ POWHEG + HERWIG ++} & \multicolumn{2}{|c|}{ MG5_aMC@NLO+PYTHIA [FxFx] } \\
\hline & $\chi^{2} /$ dof & $p$-value & $\chi^{2} /$ dof & $p$-value & $\chi^{2} /$ dof & $p$-value \\
\hline$p_{\mathrm{T}}^{\mathrm{t}}$ & $51 / 6$ & $<10^{-3}$ & $8 / 6$ & 0.239 & $18 / 6$ & 0.007 \\
\hline$p_{\mathrm{T}}^{\overline{\mathrm{t}}}$ & $41 / 6$ & $<10^{-3}$ & $9 / 6$ & 0.157 & $14 / 6$ & 0.026 \\
\hline$p_{\mathrm{T}}^{\mathrm{t}}$ (leading) & $47 / 6$ & $<10^{-3}$ & $4 / 6$ & 0.627 & $20 / 6$ & 0.003 \\
\hline$p_{\mathrm{T}}^{\mathrm{t}}$ (trailing) & $38 / 6$ & $<10^{-3}$ & $16 / 6$ & 0.012 & $9 / 6$ & 0.150 \\
\hline$p_{\mathrm{T}}^{\mathrm{t}}(\mathrm{t} \overline{\mathrm{t}} \mathrm{RF})$ & $40 / 6$ & $<10^{-3}$ & $11 / 6$ & 0.077 & $13 / 6$ & 0.046 \\
\hline$y_{\mathrm{t}}$ & $5 / 10$ & 0.864 & $5 / 10$ & 0.885 & $4 / 10$ & 0.936 \\
\hline$y_{\overline{\mathrm{t}}}$ & $2 / 10$ & 0.991 & $2 / 10$ & 0.992 & $3 / 10$ & 0.983 \\
\hline$y_{\mathrm{t}}$ (leading) & $3 / 8$ & 0.948 & $2 / 8$ & 0.966 & $3 / 8$ & 0.924 \\
\hline$y_{\mathrm{t}}$ (trailing) & $3 / 8$ & 0.956 & $3 / 8$ & 0.912 & $2 / 8$ & 0.976 \\
\hline$\Delta|y|(\mathrm{t}, \overline{\mathrm{t}})$ & $1 / 8$ & 0.995 & $3 / 8$ & 0.902 & $4 / 8$ & 0.849 \\
\hline$\Delta \phi(\mathrm{t}, \overline{\mathrm{t}})$ & $0 / 4$ & 0.980 & $3 / 4$ & 0.607 & $2 / 4$ & 0.741 \\
\hline$p_{\mathrm{T}}^{\mathrm{t} \overline{\mathrm{t}}}$ & $22 / 6$ & 0.001 & $36 / 6$ & $<10^{-3}$ & $12 / 6$ & 0.054 \\
\hline$y_{\mathrm{t} \overline{\mathrm{t}}}$ & $4 / 10$ & 0.967 & $4 / 10$ & 0.945 & $5 / 10$ & 0.891 \\
\hline$m_{\mathrm{t} \overline{\mathrm{t}}}$ & $12 / 7$ & 0.109 & $3 / 7$ & 0.906 & $6 / 7$ & 0.533 \\
\hline
\end{tabular}

Table 51. The $\chi^{2} /$ dof and $p$-values quantifying the agreement between the three MC predictions and the measured, parton-level absolute cross sections are shown.

\begin{tabular}{|c|c|c|c|c|c|c|c|c|c|c|}
\hline & \multicolumn{2}{|c|}{$\begin{array}{l}\mathrm{NNLO}+\alpha_{\mathrm{EW}}^{3} \\
(\mathrm{LUXQED} 17)\end{array}$} & \multicolumn{2}{|c|}{$\begin{array}{l}\mathrm{NNLO}+\alpha_{\mathrm{EW}}^{3} \\
(\mathrm{LUXQED} 17)\end{array}$} & \multicolumn{2}{|c|}{$\begin{array}{l}\mathrm{NNLO}+\alpha_{\mathrm{EW}}^{3} \\
(\mathrm{NNPDF} 3.1)\end{array}$} & \multicolumn{2}{|c|}{$\begin{array}{c}\text { NNLO+NNLL' } \\
(\mathrm{NNPDF} 3.1)\end{array}$} & \multicolumn{2}{|c|}{$\begin{array}{c}\text { NNLO+NNLL' } \\
(\mathrm{NNPDF} 3.1)\end{array}$} \\
\hline & $m_{\mathrm{t}}=$ & $73.3 \mathrm{Ge}$ & $m_{\mathrm{t}}=$ & 25 & $m_{\mathrm{t}}=$ & $73.3 \mathrm{GeV}$ & $m_{\mathrm{t}}$ & 3 & $m_{\mathrm{t}}=$ & $2.5 \mathrm{GeV}$ \\
\hline & $\chi^{2} /$ dof & $p$-value & $\chi^{2} /$ dof & $p$-value & $\chi^{2} / \operatorname{dof}$ & $p$-value & $\chi^{2} / \operatorname{dof}$ & $p$-value & $\chi^{2} /$ dof & $p$-value \\
\hline$p_{\mathrm{T}}^{\mathrm{t}}$ & $14 / 6$ & 0.026 & $12 / 6$ & 0.071 & $10 / 6$ & 0.115 & $17 / 6$ & 0.010 & $14 / 6$ & 0.032 \\
\hline$p_{\mathrm{T}}^{\overline{\mathrm{t}}}$ & $14 / 6$ & 0.027 & $12 / 6$ & 0.070 & $10 / 6$ & 0.122 & $13 / 6$ & 0.047 & $11 / 6$ & 0.098 \\
\hline$y_{\mathrm{t}}$ & $9 / 10$ & 0.510 & $7 / 10$ & 0.69 & $6 / 10$ & 0.787 & - & - & - & - \\
\hline$y_{\overline{\mathrm{t}}}$ & $5 / 10$ & 0.912 & $5 / 10$ & 0.877 & $3 / 10$ & 0.990 & - & - & - & - \\
\hline$p_{\mathrm{T}}^{\mathrm{tt}}$ & $18 / 6$ & 0.006 & $17 / 6$ & 0 & $14 / 6$ & 30 & - & - & - & - \\
\hline$y_{\mathrm{t} \overline{\mathrm{t}}}$ & $9 / 10$ & 0.548 & $8 / 10$ & 0.666 & $4 / 10$ & 0.937 & - & - & - & - \\
\hline$m_{\mathrm{t} \overline{\mathrm{t}}}$ & $22 / 7$ & 0.003 & $17 / 7$ & 0.015 & $16 / 7$ & 0.024 & $16 / 7$ & 0.029 & $11 / 7$ & 0.126 \\
\hline$\Delta|y|(\mathrm{t}, \overline{\mathrm{t}})$ & $6 / 8$ & 0.687 & $6 / 8$ & 0.667 & $2 / 8$ & 0.971 & - & - & - & - \\
\hline
\end{tabular}

Table 52. The $\chi^{2} /$ dof and $p$-values quantifying the agreement between the five theoretical predictions with $\mathrm{NNLO}+\alpha_{\mathrm{EW}}^{3}$ and NNLO+NNLL' precision and the measured, parton-level absolute cross sections are shown. 


\begin{tabular}{|ccccc|}
\hline & $\mathrm{aN}^{3} \mathrm{LO}$ & aNNLO \\
& $(\mathrm{NNPDF} 3.0)$ & $(\mathrm{CT} 14 \mathrm{NNLO})$ \\
& $m_{\mathrm{t}}=172.5 \mathrm{GeV}$ & $m_{\mathrm{t}}=172.5 \mathrm{GeV}$ \\
& $\chi^{2} /$ dof & $p$-value & $\chi^{2} /$ dof & $p$-value \\
\hline$p_{\mathrm{T}}^{\mathrm{t}}$ & $8 / 6$ & 0.220 & $306 / 6$ & $<10^{-3}$ \\
$y_{\mathrm{t}}$ & $6 / 10$ & 0.838 & $1038 / 10$ & $<10^{-3}$ \\
\hline
\end{tabular}

Table 53. The $\chi^{2} /$ dof and $p$-values quantifying the agreement between the two theoretical predictions with $\mathrm{aN}^{3} \mathrm{LO}$ and aNNLO precision and the measured, parton-level absolute cross sections are shown. 


\begin{tabular}{|c|c|c|c|c|c|c|}
\hline & \multicolumn{2}{|c|}{ POWHEG+PYTHIA } & \multicolumn{2}{|c|}{ POWHEG + HERWIG ++} & \multicolumn{2}{|c|}{ MG5_aMC@NLO+PYTHIA[FXFX] } \\
\hline & $\chi^{2} /$ dof & $p$-value & $\chi^{2} /$ dof & $p$-value & $\chi^{2} /$ dof & $p$-value \\
\hline$p_{\mathrm{T}}^{\mathrm{t}}$ & $47 / 5$ & $<10^{-3}$ & $3 / 5$ & 0.710 & $19 / 5$ & 0.002 \\
\hline$p_{\mathrm{T}}^{\overline{\mathrm{t}}}$ & $41 / 5$ & $<10^{-3}$ & $3 / 5$ & 0.630 & $18 / 5$ & 0.003 \\
\hline$p_{\mathrm{T}}^{\mathrm{t}}$ (leading) & $49 / 5$ & $<10^{-3}$ & $3 / 5$ & 0.635 & $24 / 5$ & $<10^{-3}$ \\
\hline$p_{\mathrm{T}}^{\mathrm{t}}($ trailing $)$ & $39 / 5$ & $<10^{-3}$ & $6 / 5$ & 0.274 & $14 / 5$ & 0.015 \\
\hline$p_{\mathrm{T}}^{\mathrm{t}}(\mathrm{t} \overline{\mathrm{t}} \mathrm{RF})$ & $36 / 5$ & $<10^{-3}$ & $7 / 5$ & 0.187 & $15 / 5$ & 0.009 \\
\hline$y_{\mathrm{t}}$ & $6 / 9$ & 0.701 & $9 / 9$ & 0.443 & $7 / 9$ & 0.639 \\
\hline$y_{\overline{\mathrm{t}}}$ & $3 / 9$ & 0.961 & $3 / 9$ & 0.952 & $3 / 9$ & 0.945 \\
\hline$y_{\mathrm{t}}$ (leading) & $3 / 7$ & 0.858 & $4 / 7$ & 0.799 & $5 / 7$ & 0.659 \\
\hline$y_{\mathrm{t}}($ trailing $)$ & $4 / 7$ & 0.826 & $5 / 7$ & 0.655 & $3 / 7$ & 0.913 \\
\hline$p_{\mathrm{T}}^{\mathrm{t} \overline{\mathrm{t}}}$ & $28 / 5$ & $<10^{-3}$ & $104 / 5$ & $<10^{-3}$ & $15 / 5$ & 0.010 \\
\hline$y_{\mathrm{t} \overline{\mathrm{t}}}$ & $3 / 9$ & 0.965 & $5 / 9$ & 0.821 & $4 / 9$ & 0.910 \\
\hline$m_{\mathrm{t} \overline{\mathrm{t}}}$ & $12 / 6$ & 0.058 & $29 / 6$ & $<10^{-3}$ & $5 / 6$ & 0.606 \\
\hline$\Delta|y|(\mathrm{t}, \overline{\mathrm{t}})$ & $1 / 7$ & 0.987 & $7 / 7$ & 0.411 & $4 / 7$ & 0.825 \\
\hline$\Delta \phi(\mathrm{t}, \overline{\mathrm{t}})$ & $0 / 3$ & 0.977 & $0 / 3$ & 0.941 & $1 / 3$ & 0.722 \\
\hline$p_{\mathrm{T}}^{\ell}$ & $87 / 4$ & $<10^{-3}$ & $2 / 4$ & 0.699 & $30 / 4$ & $<10^{-3}$ \\
\hline$p_{\mathrm{T}}^{\bar{\ell}}$ & $36 / 4$ & $<10^{-3}$ & $1 / 4$ & 0.915 & $10 / 4$ & 0.047 \\
\hline$p_{\mathrm{T}}^{\ell}$ (leading) & $112 / 4$ & $<10^{-3}$ & $2 / 4$ & 0.794 & $36 / 4$ & $<10^{-3}$ \\
\hline$p_{\mathrm{T}}^{\ell}$ (trailing) & $32 / 4$ & $<10^{-3}$ & $4 / 4$ & 0.396 & $10 / 4$ & 0.034 \\
\hline$\eta_{\ell}$ & $18 / 15$ & 0.238 & $23 / 15$ & 0.094 & $22 / 15$ & 0.119 \\
\hline$\eta_{\bar{\ell}}$ & $29 / 15$ & 0.015 & $31 / 15$ & 0.008 & $37 / 15$ & 0.001 \\
\hline$\eta_{\ell}$ (leading) & $13 / 15$ & 0.582 & $13 / 15$ & 0.565 & $21 / 15$ & 0.142 \\
\hline$\eta_{\ell}$ (trailing) & $22 / 15$ & 0.098 & $32 / 15$ & 0.007 & $27 / 15$ & 0.028 \\
\hline$p_{\mathrm{T}}^{\ell \bar{\ell}}$ & $14 / 6$ & 0.027 & $14 / 6$ & 0.034 & $7 / 6$ & 0.302 \\
\hline$m_{\ell \bar{\ell}}$ & $34 / 7$ & $<10^{-3}$ & $3 / 7$ & 0.887 & $5 / 7$ & 0.648 \\
\hline$\Delta \phi(\ell, \bar{\ell})$ & $31 / 9$ & $<10^{-3}$ & $16 / 9$ & 0.063 & $12 / 9$ & 0.233 \\
\hline$\Delta \eta(\ell, \bar{\ell})$ & $5 / 9$ & 0.815 & $4 / 9$ & 0.887 & $6 / 9$ & 0.690 \\
\hline$p_{\mathrm{T}}^{\mathrm{b}}$ (leading) & $31 / 4$ & $<10^{-3}$ & $14 / 4$ & 0.006 & $14 / 4$ & 0.006 \\
\hline$p_{\mathrm{T}}^{\mathrm{b}}$ (trailing) & $27 / 4$ & $<10^{-3}$ & $18 / 4$ & 0.001 & $11 / 4$ & 0.029 \\
\hline$\eta_{\mathrm{b}}$ (leading) & $10 / 7$ & 0.186 & $13 / 7$ & 0.082 & $8 / 7$ & 0.295 \\
\hline$\eta_{\mathrm{b}}$ (trailing) & $12 / 7$ & 0.114 & $14 / 7$ & 0.047 & $9 / 7$ & 0.227 \\
\hline$p_{\mathrm{T}}^{\mathrm{b} \overline{\mathrm{b}}}$ & $14 / 4$ & 0.007 & $6 / 4$ & 0.229 & $9 / 4$ & 0.071 \\
\hline$m_{\mathrm{b} \overline{\mathrm{b}}}$ & $3 / 3$ & 0.393 & $17 / 3$ & $<10^{-3}$ & $1 / 3$ & 0.753 \\
\hline$N_{\text {jets }}$ & $13 / 5$ & 0.025 & $38 / 5$ & $<10^{-3}$ & $36 / 5$ & $<10^{-3}$ \\
\hline
\end{tabular}

Table 54. The $\chi^{2} /$ dof and $p$-values quantifying the agreement between the three MC predictions and the measured, particle-level normalised cross sections are shown. 


\begin{tabular}{|c|c|c|c|c|c|c|}
\hline & \multicolumn{2}{|c|}{ POWHEG+PYTHIA } & \multicolumn{2}{|c|}{ POWHEG + HERWIG ++} & \multicolumn{2}{|c|}{ MG5_aMC@NLO+PYTHIA[FXFX] } \\
\hline & $\chi^{2} /$ dof & $p$-value & $\chi^{2} /$ dof & $p$-value & $\chi^{2} /$ dof & $p$-value \\
\hline$p_{\mathrm{T}}^{\mathrm{t}}$ & $52 / 6$ & $<10^{-3}$ & $3 / 6$ & 0.830 & $17 / 6$ & 0.008 \\
\hline$p_{\mathrm{T}}^{\overline{\mathrm{t}}}$ & $44 / 6$ & $<10^{-3}$ & $3 / 6$ & 0.786 & $16 / 6$ & 0.012 \\
\hline$p_{\mathrm{T}}^{\mathrm{t}}$ (leading) & $50 / 6$ & $<10^{-3}$ & $3 / 6$ & 0.756 & $21 / 6$ & 0.002 \\
\hline$p_{\mathrm{T}}^{\mathrm{t}}($ trailing $)$ & $39 / 6$ & $<10^{-3}$ & $5 / 6$ & 0.576 & $11 / 6$ & 0.099 \\
\hline$p_{\mathrm{T}}^{\mathrm{t}}(\mathrm{t} \overline{\mathrm{t}} \mathrm{RF})$ & $38 / 6$ & $<10^{-3}$ & $4 / 6$ & 0.710 & $12 / 6$ & 0.053 \\
\hline$y_{\mathrm{t}}$ & $6 / 10$ & 0.785 & $8 / 10$ & 0.627 & $6 / 10$ & 0.795 \\
\hline$y_{\overline{\mathrm{t}}}$ & $3 / 10$ & 0.989 & $3 / 10$ & 0.981 & $3 / 10$ & 0.983 \\
\hline$y_{\mathrm{t}}$ (leading) & $3 / 8$ & 0.927 & $4 / 8$ & 0.894 & $4 / 8$ & 0.840 \\
\hline$y_{\mathrm{t}}($ trailing $)$ & $3 / 8$ & 0.939 & $4 / 8$ & 0.850 & $2 / 8$ & 0.973 \\
\hline$p_{\mathrm{T}}^{\mathrm{t} \overline{\mathrm{t}}}$ & $34 / 6$ & $<10^{-3}$ & $29 / 6$ & $<10^{-3}$ & $17 / 6$ & 0.011 \\
\hline$y_{\mathrm{t} \overline{\mathrm{t}}}$ & $3 / 10$ & 0.968 & $6 / 10$ & 0.776 & $5 / 10$ & 0.858 \\
\hline$m_{\mathrm{t} \overline{\mathrm{t}}}$ & $12 / 7$ & 0.095 & $11 / 7$ & 0.135 & $5 / 7$ & 0.676 \\
\hline$\Delta|y|(\mathrm{t}, \overline{\mathrm{t}})$ & $1 / 8$ & 0.994 & $6 / 8$ & 0.595 & $3 / 8$ & 0.907 \\
\hline$\Delta \phi(\mathrm{t}, \overline{\mathrm{t}})$ & $0 / 4$ & 0.975 & $0 / 4$ & 0.982 & $2 / 4$ & 0.823 \\
\hline$p_{\mathrm{T}}^{\ell}$ & $36 / 5$ & $<10^{-3}$ & $3 / 5$ & 0.771 & $11 / 5$ & 0.059 \\
\hline$p_{\mathrm{T}}^{\bar{\ell}}$ & $35 / 5$ & $<10^{-3}$ & $1 / 5$ & 0.951 & $9 / 5$ & 0.095 \\
\hline$p_{\mathrm{T}}^{\ell}$ (leading) & $41 / 5$ & $<10^{-3}$ & $3 / 5$ & 0.731 & $12 / 5$ & 0.039 \\
\hline$p_{\mathrm{T}}^{\ell}$ (trailing) & $33 / 5$ & $<10^{-3}$ & $5 / 5$ & 0.404 & $6 / 5$ & 0.318 \\
\hline$\eta_{\ell}$ & $18 / 16$ & 0.339 & $20 / 16$ & 0.207 & $20 / 16$ & 0.234 \\
\hline$\eta_{\bar{\ell}}$ & $29 / 16$ & 0.024 & $28 / 16$ & 0.033 & $33 / 16$ & 0.007 \\
\hline$\eta_{\ell}$ (leading) & $11 / 16$ & 0.781 & $12 / 16$ & 0.766 & $17 / 16$ & 0.388 \\
\hline$\eta_{\ell}$ (trailing) & $21 / 16$ & 0.194 & $29 / 16$ & 0.025 & $24 / 16$ & 0.092 \\
\hline$p_{\mathrm{T}}^{\ell \bar{\ell}}$ & $14 / 7$ & 0.053 & $11 / 7$ & 0.120 & $6 / 7$ & 0.524 \\
\hline$m_{\ell \bar{\ell}}$ & $37 / 8$ & $<10^{-3}$ & $3 / 8$ & 0.948 & $4 / 8$ & 0.810 \\
\hline$\Delta \phi(\ell, \bar{\ell})$ & $27 / 10$ & 0.003 & $13 / 10$ & 0.235 & $10 / 10$ & 0.478 \\
\hline$\Delta \eta(\ell, \bar{\ell})$ & $6 / 10$ & 0.848 & $4 / 10$ & 0.930 & $6 / 10$ & 0.842 \\
\hline$p_{\mathrm{T}}^{\mathrm{b}}$ (leading) & $35 / 5$ & $<10^{-3}$ & $10 / 5$ & 0.085 & $17 / 5$ & 0.004 \\
\hline$p_{\mathrm{T}}^{\mathrm{b}}$ (trailing) & $27 / 5$ & $<10^{-3}$ & $10 / 5$ & 0.063 & $11 / 5$ & 0.050 \\
\hline$\eta_{\mathrm{b}}$ (leading) & $10 / 8$ & 0.251 & $11 / 8$ & 0.188 & $8 / 8$ & 0.452 \\
\hline$\eta_{\mathrm{b}}$ (trailing) & $11 / 8$ & 0.204 & $12 / 8$ & 0.151 & $8 / 8$ & 0.456 \\
\hline$p_{\mathrm{T}}^{\mathrm{b} \overline{\mathrm{b}}}$ & $12 / 5$ & 0.030 & $9 / 5$ & 0.096 & $7 / 5$ & 0.232 \\
\hline$m_{\mathrm{b} \overline{\mathrm{b}}}$ & $3 / 4$ & 0.584 & $16 / 4$ & 0.003 & $1 / 4$ & 0.897 \\
\hline$N_{\text {jets }}$ & $14 / 6$ & 0.035 & $37 / 6$ & $<10^{-3}$ & $18 / 6$ & 0.006 \\
\hline
\end{tabular}

Table 55. The $\chi^{2} /$ dof and $p$-values quantifying the agreement between the three MC predictions and the measured, particle-level absolute cross sections are shown. 
Open Access. This article is distributed under the terms of the Creative Commons Attribution License (CC-BY 4.0), which permits any use, distribution and reproduction in any medium, provided the original author(s) and source are credited.

\section{References}

[1] ATLAS collaboration, Measurements of top quark pair relative differential cross-sections with ATLAS in pp collisions at $\sqrt{s}=7$ TeV, Eur. Phys. J. C 73 (2013) 2261 [arXiv: 1207.5644] [INSPIRE].

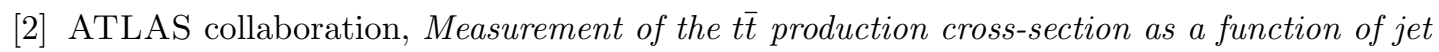
multiplicity and jet transverse momentum in 7 TeV proton-proton collisions with the ATLAS detector, JHEP 01 (2015) 020 [arXiv:1407.0891] [INSPIRE].

[3] CMS collaboration, Measurement of differential top-quark pair production cross sections in pp colisions at $\sqrt{s}=7 \mathrm{TeV}$, Eur. Phys. J. C 73 (2013) 2339 [arXiv:1211.2220] [inSPIRE].

[4] CMS collaboration, Measurement of the differential cross sections for top quark pair production as a function of kinematic event variables in pp collisions at $\sqrt{s}=7$ and $8 \mathrm{TeV}$, Phys. Rev. D 94 (2016) 052006 [arXiv: 1607.00837] [INSPIRE].

[5] ATLAS collaboration, Measurements of top-quark pair differential cross-sections in the lepton + jets channel in pp collisions at $\sqrt{s}=8 \mathrm{TeV}$ using the ATLAS detector, Eur. Phys. J. C 76 (2016) 538 [arXiv:1511.04716] [INSPIRE].

[6] ATLAS collaboration, Measurement of lepton differential distributions and the top quark mass in $t \bar{t}$ production in pp collisions at $\sqrt{s}=8 \mathrm{TeV}$ with the ATLAS detector, Eur. Phys. J. C 77 (2017) 804 [arXiv:1709.09407] [InSPIRE].

[7] ATLAS collaboration, Measurement of the differential cross-section of highly boosted top quarks as a function of their transverse momentum in $\sqrt{s}=8 \mathrm{TeV}$ proton-proton collisions using the ATLAS detector, Phys. Rev. D 93 (2016) 032009 [arXiv:1510.03818] [InSPIRE].

[8] CMS collaboration, Measurement of the differential cross section for top quark pair production in pp collisions at $\sqrt{s}=8 \mathrm{TeV}$, Eur. Phys. J. C 75 (2015) 542 [arXiv: 1505.04480] [INSPIRE].

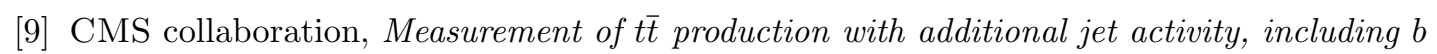
quark jets, in the dilepton decay channel using pp collisions at $\sqrt{s}=8 \mathrm{TeV}$, Eur. Phys. J. C 76 (2016) 379 [arXiv:1510.03072] [INSPIRE].

[10] CMS collaboration, Measurement of the t $\bar{t}$ production cross section in the all-jets final state in pp collisions at $\sqrt{s}=8 \mathrm{TeV}$, Eur. Phys. J. C 76 (2016) 128 [arXiv:1509.06076] [INSPIRE].

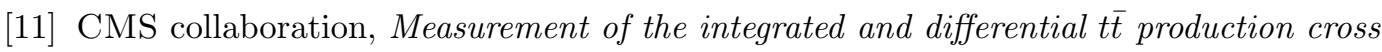
sections for high-p $p_{t}$ top quarks in pp collisions at $\sqrt{s}=8 \mathrm{TeV}$, Phys. Rev. D 94 (2016) 072002 [arXiv: 1605.00116] [INSPIRE].

[12] CMS collaboration, Measurement of double-differential cross sections for top quark pair production in pp collisions at $\sqrt{s}=8 \mathrm{TeV}$ and impact on parton distribution functions, Eur. Phys. J. C 77 (2017) 459 [arXiv: 1703.01630] [INSPIRE].

[13] ATLAS collaboration, Measurements of top-quark pair differential cross-sections in the lepton + jets channel in pp collisions at $\sqrt{s}=13 \mathrm{TeV}$ using the ATLAS detector, JHEP 11 (2017) 191 [arXiv:1708.00727] [INSPIRE]. 
[14] ATLAS collaboration, Measurement of jet activity produced in top-quark events with an electron, a muon and two b-tagged jets in the final state in pp collisions at $\sqrt{s}=13 \mathrm{TeV}$ with the ATLAS detector, Eur. Phys. J. C 77 (2017) 220 [arXiv:1610.09978] [INSPIRE].

[15] ATLAS collaboration, Measurements of top-quark pair differential cross-sections in the e $\mu$ channel in pp collisions at $\sqrt{s}=13 \mathrm{TeV}$ using the ATLAS detector, Eur. Phys. J. C 77 (2017) 292 [arXiv: 1612.05220] [INSPIRE].

[16] CMS collaboration, Measurement of differential cross sections for top quark pair production using the lepton + jets final state in proton-proton collisions at 13 TeV, Phys. Rev. D 95 (2017) 092001 [arXiv: 1610.04191] [INSPIRE].

[17] CMS collaboration, Measurement of differential cross sections for the production of top quark pairs and of additional jets in lepton + jets events from pp collisions at $\sqrt{s}=13 \mathrm{TeV}$, Phys. Rev. D 97 (2018) 112003 [arXiv: 1803.08856] [INSPIRE].

[18] CMS collaboration, Measurement of normalized differential $t \bar{t}$ cross sections in the dilepton channel from pp collisions at $\sqrt{s}=13 \mathrm{TeV}$, JHEP 04 (2018) 060 [arXiv:1708.07638] [INSPIRE].

[19] CMS collaboration, Measurements of differential cross sections of top quark pair production as a function of kinematic event variables in proton-proton collisions at $\sqrt{s}=13 \mathrm{TeV}$, JHEP 06 (2018) 002 [arXiv: 1803.03991] [INSPIRE].

[20] CMS collaboration, CMS Luminosity Measurements for the 2016 Data Taking Period, CMS-PAS-LUM-17-001 (2017) [INSPIRE].

[21] M. Guzzi, K. Lipka and S.-O. Moch, Top-quark pair production at hadron colliders: differential cross section and phenomenological applications with DiffTop, JHEP 01 (2015) 082 [arXiv: 1406.0386] [INSPIRE].

[22] N. Kidonakis, NNNLO soft-gluon corrections for the top-quark $p_{T}$ and rapidity distributions, Phys. Rev. D 91 (2015) 031501 [arXiv:1411.2633] [INSPIRE].

[23] M. Czakon, D. Heymes and A. Mitov, High-precision differential predictions for top-quark pairs at the LHC, Phys. Rev. Lett. 116 (2016) 082003 [arXiv: 1511.00549] [INSPIRE].

[24] B.D. Pecjak, D.J. Scott, X. Wang and L.L. Yang, Resummed differential cross sections for top-quark pairs at the LHC, Phys. Rev. Lett. 116 (2016) 202001 [arXiv:1601. 07020] [INSPIRE].

[25] X.-Q. Li, Z.-G. Si, K. Wang, L. Wang, L. Zhang and G. Zhu, Light Top Squark in Precision Top Quark Sample, Phys. Rev. D 89 (2014) 077703 [arXiv:1311.6874] [INSPIRE].

[26] R. Frederix and F. Maltoni, Top pair invariant mass distribution: A Window on new physics, JHEP 01 (2009) 047 [arXiv: 0712 .2355] [INSPIRE].

[27] R.M. Harris and S. Jain, Cross Sections for Leptophobic Topcolor Z' Decaying to Top-Antitop, Eur. Phys. J. C 72 (2012) 2072 [arXiv:1112.4928] [INSPIRE].

[28] D. Buarque Franzosi and C. Zhang, Probing the top-quark chromomagnetic dipole moment at next-to-leading order in QCD, Phys. Rev. D 91 (2015) 114010 [arXiv:1503.08841] [INSPIRE].

[29] R. Martinez, M.A. Perez and N. Poveda, Chromomagnetic Dipole Moment of the Top Quark Revisited, Eur. Phys. J. C 53 (2008) 221 [hep-ph/0701098] [INSPIRE]. 
[30] CMS collaboration, The CMS trigger system, 2017 JINST 12 P01020 [arXiv:1609.02366] [INSPIRE].

[31] CMS collaboration, The CMS Experiment at the CERN LHC, 2008 JINST 3 S08004 [INSPIRE].

[32] S. Frixione, P. Nason and G. Ridolfi, A Positive-weight next-to-leading-order Monte Carlo for heavy flavour hadroproduction, JHEP 09 (2007) 126 [arXiv:0707.3088] [INSPIRE].

[33] P. Nason, A New method for combining NLO QCD with shower Monte Carlo algorithms, JHEP 11 (2004) 040 [hep-ph/0409146] [INSPIRE].

[34] S. Frixione, P. Nason and C. Oleari, Matching NLO QCD computations with Parton Shower simulations: the POWHEG method, JHEP 11 (2007) 070 [arXiv:0709.2092] [INSPIRE].

[35] S. Alioli, P. Nason, C. Oleari and E. Re, A general framework for implementing NLO calculations in shower Monte Carlo programs: the POWHEG BOX, JHEP 06 (2010) 043 [arXiv: 1002.2581] [INSPIRE].

[36] CMS collaboration, Investigations of the impact of the parton shower tuning in PYTHIA 8 in the modelling of $t \bar{t}$ at $\sqrt{s}=8$ and $13 \mathrm{TeV}$, CMS-PAS-TOP-16-021 (2016) [INSPIRE].

[37] T. Sjöstrand et al., An Introduction to PYTHIA 8.2, Comput. Phys. Commun. 191 (2015) 159 [arXiv: 1410.3012] [INSPIRE].

[38] CMS collaboration, Event generator tunes obtained from underlying event and multiparton scattering measurements, Eur. Phys. J. C 76 (2016) 155 [arXiv:1512.00815] [INSPIRE].

[39] P. Skands, S. Carrazza and J. Rojo, Tuning PYTHIA 8.1: the Monash 2013 Tune, Eur. Phys. J. C 74 (2014) 3024 [arXiv: 1404.5630] [INSPIRE].

[40] J. Alwall et al., The automated computation of tree-level and next-to-leading order differential cross sections and their matching to parton shower simulations, JHEP 07 (2014) 079 [arXiv: 1405.0301] [INSPIRE].

[41] P. Artoisenet, R. Frederix, O. Mattelaer and R. Rietkerk, Automatic spin-entangled decays of heavy resonances in Monte Carlo simulations, JHEP 03 (2013) 015 [arXiv:1212.3460] [INSPIRE].

[42] R. Frederix and S. Frixione, Merging meets matching in MC@NLO, JHEP 12 (2012) 061 [arXiv: 1209.6215] [INSPIRE].

[43] M. Bahr et al., HERWIG++ Physics and Manual, Eur. Phys. J. C 58 (2008) 639 [arXiv: 0803.0883] [INSPIRE].

[44] M.H. Seymour and A. Siodmok, Constraining MPI models using $\sigma_{\mathrm{eff}}$ and recent Tevatron and LHC Underlying Event data, JHEP 10 (2013) 113 [arXiv:1307.5015] [INSPIRE].

[45] J. Alwall et al., Comparative study of various algorithms for the merging of parton showers and matrix elements in hadronic collisions, Eur. Phys. J. C 53 (2008) 473 [arXiv:0706.2569] [INSPIRE].

[46] S. Alioli, P. Nason, C. Oleari and E. Re, NLO single-top production matched with shower in POWHEG: s- and t-channel contributions, JHEP 09 (2009) 111 [Erratum JHEP 02 (2010) 011] [arXiv: 0907.4076] [INSPIRE].

[47] E. Re, Single-top Wt-channel production matched with parton showers using the POWHEG method, Eur. Phys. J. C 71 (2011) 1547 [arXiv:1009.2450] [inSPIRE]. 
[48] NNPDF collaboration, Unbiased global determination of parton distributions and their uncertainties at NNLO and at LO, Nucl. Phys. B 855 (2012) 153 [arXiv:1107.2652] [INSPIRE].

[49] Y. Li and F. Petriello, Combining QCD and electroweak corrections to dilepton production in FEWZ, Phys. Rev. D 86 (2012) 094034 [arXiv: 1208.5967] [InSPIRE].

[50] N. Kidonakis, Two-loop soft anomalous dimensions for single top quark associated production with a $W^{-}$or $H^{-}$, Phys. Rev. D 82 (2010) 054018 [arXiv:1005.4451] [INSPIRE].

[51] J.M. Campbell, R.K. Ellis and C. Williams, Vector boson pair production at the LHC, JHEP 07 (2011) 018 [arXiv: 1105.0020] [INSPIRE].

[52] F. Maltoni, D. Pagani and I. Tsinikos, Associated production of a top-quark pair with vector bosons at NLO in QCD: impact on $t \bar{t} H$ searches at the LHC, JHEP 02 (2016) 113 [arXiv: 1507.05640] [INSPIRE].

[53] M. Czakon and A. Mitov, Top++: A Program for the Calculation of the Top-Pair Cross-Section at Hadron Colliders, Comput. Phys. Commun. 185 (2014) 2930 [arXiv:1112.5675] [INSPIRE].

[54] GEANT4 collaboration, GEANT4: A Simulation toolkit, Nucl. Instrum. Meth. A 506 (2003) 250 [INSPIRE].

[55] CMS collaboration, Particle-flow reconstruction and global event description with the CMS detector, 2017 JINST 12 P10003 [arXiv:1706.04965] [INSPIRE].

[56] CMS collaboration, Performance of Electron Reconstruction and Selection with the CMS Detector in Proton-Proton Collisions at $\sqrt{s}=8 \mathrm{TeV}, 2015$ JINST $10 \mathrm{P} 06005$ [arXiv: 1502.02701] [INSPIRE].

[57] CMS collaboration, Performance of the CMS muon detector and muon reconstruction with proton-proton collisions at $\sqrt{s}=13$ TeV, 2018 JINST 13 P06015 [arXiv:1804.04528] [INSPIRE].

[58] M. Cacciari, G.P. Salam and G. Soyez, The anti- $k_{t}$ jet clustering algorithm, JHEP 04 (2008) 063 [arXiv:0802.1189] [INSPIRE].

[59] M. Cacciari, G.P. Salam and G. Soyez, FastJet User Manual, Eur. Phys. J. C 72 (2012) 1896 [arXiv:1111.6097] [INSPIRE].

[60] CMS collaboration, Identification of heavy-flavour jets with the CMS detector in pp collisions at $13 \mathrm{TeV}, 2018$ JINST 13 P05011 [arXiv: 1712.07158] [INSPIRE].

[61] CMS collaboration, Measurement of the top quark pair production cross section in proton-proton collisions at $\sqrt{s}=13$ TeV, Phys. Rev. Lett. 116 (2016) 052002 [arXiv: 1510.05302] [INSPIRE].

[62] Particle Data Group collaboration, Review of Particle Physics, Chin. Phys. C 40 (2016) 100001 [INSPIRE].

[63] A. Hocker and V. Kartvelishvili, SVD approach to data unfolding, Nucl. Instrum. Meth. A 372 (1996) 469 [hep-ph/9509307] [INSPIRE].

[64] V. Blobel, An Unfolding method for high-energy physics experiments, in proceedings of the Conference on Advanced Statistical Techniques in Particle Physics, Durham, U.K., 18-22 March 2002, pp. 258-267 [hep-ex/0208022] [InSPIRE] and online pdf version at http://www.ippp.dur.ac.uk/Workshops/02/statistics/proceedings//blobel2.pdf. 
[65] F. James, Statistical methods in experimental physics, second edition, World Scientific (2006).

[66] CMS collaboration, Object definitions for top quark analyses at the particle level, CMS-NOTE-2017-004 (2017).

[67] CMS collaboration, Measurement of the Drell-Yan Cross Section in pp Collisions at $\sqrt{s}=7 \mathrm{TeV}$, JHEP 10 (2011) 007 [arXiv:1108.0566] [INSPIRE].

[68] CMS collaboration, Jet energy scale and resolution in the CMS experiment in pp collisions at $8 \mathrm{TeV}, 2017$ JINST $12 \mathrm{P} 02014$ [arXiv: 1607.03663] [INSPIRE].

[69] ATLAS collaboration, Measurement of the Inelastic Proton-Proton Cross Section at $\sqrt{s}=13$ TeV with the ATLAS Detector at the LHC, Phys. Rev. Lett. 117 (2016) 182002 [arXiv: 1606.02625] [INSPIRE].

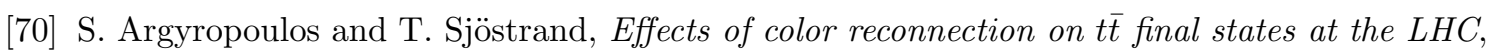
JHEP 11 (2014) 043 [arXiv: 1407.6653] [INSPIRE].

[71] J.R. Christiansen and P.Z. Skands, String Formation Beyond Leading Colour, JHEP 08 (2015) 003 [arXiv: 1505.01681] [InSPIRE].

[72] M.G. Bowler, $e^{+} e^{-}$Production of Heavy Quarks in the String Model, Z. Phys. C 11 (1981) 169 [INSPIRE].

[73] C. Peterson, D. Schlatter, I. Schmitt and P.M. Zerwas, Scaling Violations in Inclusive $e^{+} e^{-}$ Annihilation Spectra, Phys. Rev. D 27 (1983) 105 [inSPIRE].

[74] M. Czakon, D. Heymes, A. Mitov, D. Pagani, I. Tsinikos and M. Zaro, Top-pair production at the LHC through NNLO QCD and NLO EW, JHEP 10 (2017) 186 [arXiv:1705.04105] [INSPIRE].

[75] A. Manohar, P. Nason, G.P. Salam and G. Zanderighi, How bright is the proton? A precise determination of the photon parton distribution function, Phys. Rev. Lett. 117 (2016) 242002 [arXiv: 1607.04266] [INSPIRE].

[76] NNPDF collaboration, Illuminating the photon content of the proton within a global PDF analysis, SciPost Phys. 5 (2018) 008 [arXiv: 1712.07053] [INSPIRE].

[77] M. Czakon et al., Resummation for (boosted) top-quark pair production at $N N L O+N N L L^{\prime}$ in QCD, JHEP 05 (2018) 149 [arXiv: 1803.07623] [INSPIRE].

[78] NNPDF collaboration, Parton distributions from high-precision collider data, Eur. Phys. J. C 77 (2017) 663 [arXiv:1706.00428] [INSPIRE].

[79] S. Dulat et al., New parton distribution functions from a global analysis of quantum chromodynamics, Phys. Rev. D 93 (2016) 033006 [arXiv: 1506.07443] [InSPIRE].

[80] W. Buchmuller and D. Wyler, Effective Lagrangian Analysis of New Interactions and Flavor Conservation, Nucl. Phys. B 268 (1986) 621 [INSPIRE].

[81] B. Grzadkowski, M. Iskrzynski, M. Misiak and J. Rosiek, Dimension-Six Terms in the Standard Model Lagrangian, JHEP 10 (2010) 085 [arXiv: 1008.4884] [INSPIRE].

[82] A. Buckley et al., Rivet user manual, Comput. Phys. Commun. 184 (2013) 2803 [arXiv: 1003.0694] [INSPIRE].

[83] M. Czakon, P. Fiedler and A. Mitov, Total Top-Quark Pair-Production Cross Section at Hadron Colliders Through $\mathcal{O}\left(\alpha_{S}^{4}\right)$, Phys. Rev. Lett. 110 (2013) 252004 [arXiv:1303.6254] [INSPIRE]. 
[84] CMS collaboration, Combination of ATLAS and CMS top quark pair cross section measurements in the e $\mu$ final state using proton-proton collisions at $8 \mathrm{TeV}$,

CMS-PAS-TOP-14-016 (2014) [INSPIRE].

[85] CDF and D0 collaborations, Combination of measurements of the top-quark pair production cross section from the Tevatron Collider, Phys. Rev. D 89 (2014) 072001 [arXiv:1309.7570] [INSPIRE].

[86] CMS collaboration, Measurements of $t \bar{t}$ spin correlations and top quark polarization using dilepton final states in pp collisions at $\sqrt{s}=8$ TeV, Phys. Rev. D 93 (2016) 052007 [arXiv: 1601.01107] [INSPIRE].

[87] W. Bernreuther and Z.-G. Si, Top quark spin correlations and polarization at the LHC: standard model predictions and effects of anomalous top chromo moments, Phys. Lett. B 725 (2013) 115 [Erratum ibid. B 744 (2015) 413] [arXiv:1305.2066] [INSPIRE].

[88] J.A. Aguilar-Saavedra and M. Pérez-Victoria, Simple models for the top asymmetry: Constraints and predictions, JHEP 09 (2011) 097 [arXiv:1107.0841] [INSPIRE].

[89] J.H. Kuhn and G. Rodrigo, Charge asymmetries of top quarks at hadron colliders revisited, JHEP 01 (2012) 063 [arXiv: 1109.6830] [INSPIRE].

[90] W. Bernreuther, D. Heisler and Z.-G. Si, A set of top quark spin correlation and polarization observables for the LHC: Standard Model predictions and new physics contributions, JHEP 12 (2015) 026 [arXiv: 1508.05271] [INSPIRE]. 


\section{The CMS collaboration}

\section{Yerevan Physics Institute, Yerevan, Armenia}

A.M. Sirunyan, A. Tumasyan

\section{Institut für Hochenergiephysik, Wien, Austria}

W. Adam, F. Ambrogi, E. Asilar, T. Bergauer, J. Brandstetter, M. Dragicevic, J. Erö, A. Escalante Del Valle, M. Flechl, R. Frühwirth ${ }^{1}$, V.M. Ghete, J. Hrubec, M. Jeitler ${ }^{1}$, N. Krammer, I. Krätschmer, D. Liko, T. Madlener, I. Mikulec, N. Rad, H. Rohringer, J. Schieck ${ }^{1}$, R. Schöfbeck, M. Spanring, D. Spitzbart, A. Taurok, W. Waltenberger, J. Wittmann, C.-E. Wulz ${ }^{1}$, M. Zarucki

\section{Institute for Nuclear Problems, Minsk, Belarus}

V. Chekhovsky, V. Mossolov, J. Suarez Gonzalez

Universiteit Antwerpen, Antwerpen, Belgium

E.A. De Wolf, D. Di Croce, X. Janssen, J. Lauwers, M. Pieters, H. Van Haevermaet, P. Van Mechelen, N. Van Remortel

\section{Vrije Universiteit Brussel, Brussel, Belgium}

S. Abu Zeid, F. Blekman, J. D'Hondt, I. De Bruyn, J. De Clercq, K. Deroover, G. Flouris, D. Lontkovskyi, S. Lowette, I. Marchesini, S. Moortgat, L. Moreels, Q. Python, K. Skovpen, S. Tavernier, W. Van Doninck, P. Van Mulders, I. Van Parijs

\section{Université Libre de Bruxelles, Bruxelles, Belgium}

D. Beghin, B. Bilin, H. Brun, B. Clerbaux, G. De Lentdecker, H. Delannoy, B. Dorney, G. Fasanella, L. Favart, R. Goldouzian, A. Grebenyuk, A.K. Kalsi, T. Lenzi, J. Luetic, N. Postiau, E. Starling, L. Thomas, C. Vander Velde, P. Vanlaer, D. Vannerom, Q. Wang

\section{Ghent University, Ghent, Belgium}

T. Cornelis, D. Dobur, A. Fagot, M. Gul, I. Khvastunov², D. Poyraz, C. Roskas, D. Trocino, M. Tytgat, W. Verbeke, B. Vermassen, M. Vit, N. Zaganidis

\section{Université Catholique de Louvain, Louvain-la-Neuve, Belgium}

H. Bakhshiansohi, O. Bondu, S. Brochet, G. Bruno, C. Caputo, P. David, C. Delaere, M. Delcourt, B. Francois, A. Giammanco, G. Krintiras, V. Lemaitre, A. Magitteri, A. Mertens, M. Musich, K. Piotrzkowski, A. Saggio, M. Vidal Marono, S. Wertz, J. Zobec

\section{Centro Brasileiro de Pesquisas Fisicas, Rio de Janeiro, Brazil}

F.L. Alves, G.A. Alves, M. Correa Martins Junior, G. Correia Silva, C. Hensel, A. Moraes, M.E. Pol, P. Rebello Teles

Universidade do Estado do Rio de Janeiro, Rio de Janeiro, Brazil

E. Belchior Batista Das Chagas, W. Carvalho, J. Chinellato ${ }^{3}$, E. Coelho, E.M. Da Costa, G.G. Da Silveira ${ }^{4}$, D. De Jesus Damiao, C. De Oliveira Martins, S. Fonseca De Souza, H. Malbouisson, D. Matos Figueiredo, M. Melo De Almeida, C. Mora Herrera, L. Mundim, H. Nogima, W.L. Prado Da Silva, L.J. Sanchez Rosas, A. Santoro, A. Sznajder, M. Thiel, E.J. Tonelli Manganote ${ }^{3}$, F. Torres Da Silva De Araujo, A. Vilela Pereira 
Universidade Estadual Paulista ${ }^{a}$, Universidade Federal do ABC ${ }^{b}$, São Paulo, Brazil

S. Ahuja ${ }^{a}$, C.A. Bernardes ${ }^{a}$, L. Calligaris $^{a}$, T.R. Fernandez Perez Tomei ${ }^{a}$, E.M. $_{\text {Gregores }}{ }^{b}$, P.G. Mercadante ${ }^{b}$, S.F. Novaes ${ }^{a}$, SandraS. Padula ${ }^{a}$

Institute for Nuclear Research and Nuclear Energy, Bulgarian Academy of Sciences, Sofia, Bulgaria

A. Aleksandrov, R. Hadjiiska, P. Iaydjiev, A. Marinov, M. Misheva, M. Rodozov,

M. Shopova, G. Sultanov

University of Sofia, Sofia, Bulgaria

A. Dimitrov, L. Litov, B. Pavlov, P. Petkov

Beihang University, Beijing, China

W. Fang ${ }^{5}$, X. Gao ${ }^{5}$, L. Yuan

Institute of High Energy Physics, Beijing, China

M. Ahmad, J.G. Bian, G.M. Chen, H.S. Chen, M. Chen, Y. Chen, C.H. Jiang, D. Leggat,

H. Liao, Z. Liu, F. Romeo, S.M. Shaheen ${ }^{6}$, A. Spiezia, J. Tao, Z. Wang, E. Yazgan,

H. Zhang, S. Zhang ${ }^{6}$, J. Zhao

State Key Laboratory of Nuclear Physics and Technology, Peking University, Beijing, China

Y. Ban, G. Chen, A. Levin, J. Li, L. Li, Q. Li, Y. Mao, S.J. Qian, D. Wang, Z. Xu

Tsinghua University, Beijing, China

Y. Wang

Universidad de Los Andes, Bogota, Colombia

C. Avila, A. Cabrera, C.A. Carrillo Montoya, L.F. Chaparro Sierra, C. Florez,

C.F. González Hernández, M.A. Segura Delgado

University of Split, Faculty of Electrical Engineering, Mechanical Engineering and Naval Architecture, Split, Croatia

B. Courbon, N. Godinovic, D. Lelas, I. Puljak, T. Sculac

University of Split, Faculty of Science, Split, Croatia

Z. Antunovic, M. Kovac

Institute Rudjer Boskovic, Zagreb, Croatia

V. Brigljevic, D. Ferencek, K. Kadija, B. Mesic, A. Starodumov ${ }^{7}$, T. Susa

University of Cyprus, Nicosia, Cyprus

M.W. Ather, A. Attikis, M. Kolosova, G. Mavromanolakis, J. Mousa, C. Nicolaou, F. Ptochos, P.A. Razis, H. Rykaczewski

Charles University, Prague, Czech Republic

M. Finger ${ }^{8}$, M. Finger Jr. ${ }^{8}$ 
Escuela Politecnica Nacional, Quito, Ecuador

E. Ayala

Universidad San Francisco de Quito, Quito, Ecuador

E. Carrera Jarrin

Academy of Scientific Research and Technology of the Arab Republic of Egypt, Egyptian Network of High Energy Physics, Cairo, Egypt

H. Abdalla ${ }^{9}$, A.A. Abdelalim ${ }^{10,11}$, E. Salama ${ }^{12,13}$

National Institute of Chemical Physics and Biophysics, Tallinn, Estonia

S. Bhowmik, A. Carvalho Antunes De Oliveira, R.K. Dewanjee, K. Ehataht, M. Kadastik, M. Raidal, C. Veelken

Department of Physics, University of Helsinki, Helsinki, Finland

P. Eerola, H. Kirschenmann, J. Pekkanen, M. Voutilainen

Helsinki Institute of Physics, Helsinki, Finland

J. Havukainen, J.K. Heikkilä, T. Järvinen, V. Karimäki, R. Kinnunen, T. Lampén, K. Lassila-Perini, S. Laurila, S. Lehti, T. Lindén, P. Luukka, T. Mäenpää, H. Siikonen, E. Tuominen, J. Tuominiemi

\section{Lappeenranta University of Technology, Lappeenranta, Finland}

T. Tuuva

IRFU, CEA, Université Paris-Saclay, Gif-sur-Yvette, France

M. Besancon, F. Couderc, M. Dejardin, D. Denegri, J.L. Faure, F. Ferri, S. Ganjour, A. Givernaud, P. Gras, G. Hamel de Monchenault, P. Jarry, C. Leloup, E. Locci, J. Malcles, G. Negro, J. Rander, A. Rosowsky, M.Ö. Sahin, M. Titov

Laboratoire Leprince-Ringuet, Ecole polytechnique, CNRS/IN2P3, Université Paris-Saclay, Palaiseau, France

A. Abdulsalam ${ }^{14}$, C. Amendola, I. Antropov, F. Beaudette, P. Busson, C. Charlot, R. Granier de Cassagnac, I. Kucher, A. Lobanov, J. Martin Blanco, C. Martin Perez, M. Nguyen, C. Ochando, G. Ortona, P. Paganini, P. Pigard, J. Rembser, R. Salerno, J.B. Sauvan, Y. Sirois, A.G. Stahl Leiton, A. Zabi, A. Zghiche

Université de Strasbourg, CNRS, IPHC UMR 7178, Strasbourg, France J.-L. Agram ${ }^{15}$, J. Andrea, D. Bloch, J.-M. Brom, E.C. Chabert, V. Cherepanov, C. Collard, E. Conte ${ }^{15}$, J.-C. Fontaine ${ }^{15}$, D. Gelé, U. Goerlach, M. Jansová, A.-C. Le Bihan, N. Tonon, P. Van Hove

Centre de Calcul de l'Institut National de Physique Nucleaire et de Physique des Particules, CNRS/IN2P3, Villeurbanne, France

S. Gadrat 
Université de Lyon, Université Claude Bernard Lyon 1, CNRS-IN2P3, Institut de Physique Nucléaire de Lyon, Villeurbanne, France

S. Beauceron, C. Bernet, G. Boudoul, N. Chanon, R. Chierici, D. Contardo, P. Depasse, H. El Mamouni, J. Fay, L. Finco, S. Gascon, M. Gouzevitch, G. Grenier, B. Ille, F. Lagarde, I.B. Laktineh, H. Lattaud, M. Lethuillier, L. Mirabito, S. Perries, A. Popov ${ }^{16}$, V. Sordini, G. Touquet, M. Vander Donckt, S. Viret

Georgian Technical University, Tbilisi, Georgia

A. Khvedelidze ${ }^{8}$

Tbilisi State University, Tbilisi, Georgia

Z. Tsamalaidze ${ }^{8}$

RWTH Aachen University, I. Physikalisches Institut, Aachen, Germany

C. Autermann, L. Feld, M.K. Kiesel, K. Klein, M. Lipinski, M. Preuten, M.P. Rauch, C. Schomakers, J. Schulz, M. Teroerde, B. Wittmer, V. Zhukov ${ }^{16}$

RWTH Aachen University, III. Physikalisches Institut A, Aachen, Germany

A. Albert, D. Duchardt, M. Endres, M. Erdmann, S. Ghosh, A. Güth, T. Hebbeker, C. Heidemann, K. Hoepfner, H. Keller, L. Mastrolorenzo, M. Merschmeyer, A. Meyer, P. Millet, S. Mukherjee, T. Pook, M. Radziej, H. Reithler, M. Rieger, A. Schmidt, D. Teyssier

RWTH Aachen University, III. Physikalisches Institut B, Aachen, Germany

G. Flügge, O. Hlushchenko, T. Kress, A. Künsken, T. Müller, A. Nehrkorn, A. Nowack, C. Pistone, O. Pooth, D. Roy, H. Sert, A. Stahl ${ }^{17}$

\section{Deutsches Elektronen-Synchrotron, Hamburg, Germany}

M. Aldaya Martin, T. Arndt, C. Asawatangtrakuldee, I. Babounikau, K. Beernaert, O. Behnke, U. Behrens, A. Bermúdez Martínez, D. Bertsche, A.A. Bin Anuar, K. Borras ${ }^{18}$, V. Botta, A. Campbell, P. Connor, C. Contreras-Campana, V. Danilov, A. De Wit, M.M. Defranchis, C. Diez Pardos, D. Domínguez Damiani, G. Eckerlin, T. Eichhorn, A. Elwood, E. Eren, E. Gallo ${ }^{19}$, A. Geiser, J.M. Grados Luyando, A. Grohsjean, M. Guthoff, M. Haranko, A. Harb, J. Hauk, H. Jung, M. Kasemann, J. Keaveney, C. Kleinwort, J. Knolle, D. Krücker, W. Lange, A. Lelek, T. Lenz, J. Leonard, K. Lipka, W. Lohmann ${ }^{20}$, R. Mankel, I.-A. Melzer-Pellmann, A.B. Meyer, M. Meyer, M. Missiroli, G. Mittag, J. Mnich, V. Myronenko, S.K. Pflitsch, D. Pitzl, A. Raspereza, M. Savitskyi, P. Saxena, P. Schütze, C. Schwanenberger, R. Shevchenko, A. Singh, H. Tholen, O. Turkot, A. Vagnerini, G.P. Van Onsem, R. Walsh, Y. Wen, K. Wichmann, C. Wissing, O. Zenaiev

\section{University of Hamburg, Hamburg, Germany}

R. Aggleton, S. Bein, L. Benato, A. Benecke, V. Blobel, T. Dreyer, E. Garutti, D. Gonzalez, P. Gunnellini, J. Haller, A. Hinzmann, A. Karavdina, G. Kasieczka, R. Klanner, R. Kogler, N. Kovalchuk, S. Kurz, V. Kutzner, J. Lange, D. Marconi, J. Multhaup, M. Niedziela, C.E.N. Niemeyer, D. Nowatschin, A. Perieanu, A. Reimers, O. Rieger, C. Scharf, P. Schleper, S. Schumann, J. Schwandt, J. Sonneveld, H. Stadie, G. Steinbrück, F.M. Stober, M. Stöver, A. Vanhoefer, B. Vormwald, I. Zoi 
Karlsruher Institut fuer Technologie, Karlsruhe, Germany

M. Akbiyik, C. Barth, M. Baselga, S. Baur, E. Butz, R. Caspart, T. Chwalek, F. Colombo, W. De Boer, A. Dierlamm, K. El Morabit, N. Faltermann, B. Freund, M. Giffels, M.A. Harrendorf, F. Hartmann ${ }^{17}$, S.M. Heindl, U. Husemann, F. Kassel ${ }^{17}$, I. Katkov ${ }^{16}$, S. Kudella, H. Mildner, S. Mitra, M.U. Mozer, Th. Müller, M. Plagge, G. Quast, K. Rabbertz, M. Schröder, I. Shvetsov, G. Sieber, H.J. Simonis, R. Ulrich, S. Wayand, M. Weber, T. Weiler, S. Williamson, C. Wöhrmann, R. Wolf

Institute of Nuclear and Particle Physics (INPP), NCSR Demokritos, Aghia Paraskevi, Greece

G. Anagnostou, G. Daskalakis, T. Geralis, A. Kyriakis, D. Loukas, G. Paspalaki, I. TopsisGiotis

National and Kapodistrian University of Athens, Athens, Greece

G. Karathanasis, S. Kesisoglou, P. Kontaxakis, A. Panagiotou, I. Papavergou, N. Saoulidou, E. Tziaferi, K. Vellidis

National Technical University of Athens, Athens, Greece

K. Kousouris, I. Papakrivopoulos, G. Tsipolitis

University of Ioánnina, Ioánnina, Greece

I. Evangelou, C. Foudas, P. Gianneios, P. Katsoulis, P. Kokkas, S. Mallios, N. Manthos, I. Papadopoulos, E. Paradas, J. Strologas, F.A. Triantis, D. Tsitsonis

MTA-ELTE Lendület CMS Particle and Nuclear Physics Group, Eötvös Loránd University, Budapest, Hungary

M. Bartók ${ }^{21}$, M. Csanad, N. Filipovic, P. Major, M.I. Nagy, G. Pasztor, O. Surányi, G.I. Veres

Wigner Research Centre for Physics, Budapest, Hungary

G. Bencze, C. Hajdu, D. Horvath² ${ }^{22}$ Á. Hunyadi, F. Sikler, T.Á. Vámi, V. Veszpremi, G. Vesztergombi ${ }^{\dagger}$

Institute of Nuclear Research ATOMKI, Debrecen, Hungary

N. Beni, S. Czellar, J. Karancsi ${ }^{23}$, A. Makovec, J. Molnar, Z. Szillasi

Institute of Physics, University of Debrecen, Debrecen, Hungary

P. Raics, Z.L. Trocsanyi, B. Ujvari

Indian Institute of Science (IISc), Bangalore, India

S. Choudhury, J.R. Komaragiri, P.C. Tiwari

National Institute of Science Education and Research, HBNI, Bhubaneswar, India

S. Bahinipati ${ }^{24}$, C. Kar, P. Mal, K. Mandal, A. $\operatorname{Nayak}^{25}$, D.K. Sahoo ${ }^{24}$, S.K. Swain 
Panjab University, Chandigarh, India

S. Bansal, S.B. Beri, V. Bhatnagar, S. Chauhan, R. Chawla, N. Dhingra, R. Gupta, A. Kaur, M. Kaur, S. Kaur, R. Kumar, P. Kumari, M. Lohan, A. Mehta, K. Sandeep, S. Sharma, J.B. Singh, A.K. Virdi, G. Walia

\section{University of Delhi, Delhi, India}

A. Bhardwaj, B.C. Choudhary, R.B. Garg, M. Gola, S. Keshri, Ashok Kumar, S. Malhotra, M. Naimuddin, P. Priyanka, K. Ranjan, Aashaq Shah, R. Sharma

Saha Institute of Nuclear Physics, HBNI, Kolkata, India

R. Bhardwaj ${ }^{26}$, M. Bharti, R. Bhattacharya, S. Bhattacharya, U. Bhawandeep ${ }^{26}$, D. Bhowmik, S. Dey, S. Dutt ${ }^{26}$, S. Dutta, S. Ghosh, K. Mondal, S. Nandan, A. Purohit, P.K. Rout, A. Roy, S. Roy Chowdhury, G. Saha, S. Sarkar, M. Sharan, B. Singh, S. Thakur ${ }^{26}$

\section{Indian Institute of Technology Madras, Madras, India}

P.K. Behera

\section{Bhabha Atomic Research Centre, Mumbai, India}

R. Chudasama, D. Dutta, V. Jha, V. Kumar, P.K. Netrakanti, L.M. Pant, P. Shukla

Tata Institute of Fundamental Research-A, Mumbai, India

T. Aziz, M.A. Bhat, S. Dugad, G.B. Mohanty, N. Sur, B. Sutar, RavindraKumar Verma

Tata Institute of Fundamental Research-B, Mumbai, India

S. Banerjee, S. Bhattacharya, S. Chatterjee, P. Das, M. Guchait, Sa. Jain, S. Karmakar, S. Kumar, M. Maity ${ }^{27}$, G. Majumder, K. Mazumdar, N. Sahoo, T. Sarkar ${ }^{27}$

Indian Institute of Science Education and Research (IISER), Pune, India

S. Chauhan, S. Dube, V. Hegde, A. Kapoor, K. Kothekar, S. Pandey, A. Rane, S. Sharma

\section{Institute for Research in Fundamental Sciences (IPM), Tehran, Iran}

S. Chenarani ${ }^{28}$, E. Eskandari Tadavani, S.M. Etesami ${ }^{28}$, M. Khakzad, M. Mohammadi Najafabadi, M. Naseri, F. Rezaei Hosseinabadi, B. Safarzadeh ${ }^{29}$, M. Zeinali

\section{University College Dublin, Dublin, Ireland}

M. Felcini, M. Grunewald

INFN Sezione di Bari ${ }^{a}$, Università di Bari ${ }^{b}$, Politecnico di Bari ${ }^{c}$, Bari, Italy M. Abbrescia ${ }^{a, b}$, C. Calabria ${ }^{a, b}$, A. Colaleo ${ }^{a}$, D. Creanza ${ }^{a, c}$, L. Cristella ${ }^{a, b}$, N. De Filippis ${ }^{a, c}$, M. De Palma ${ }^{a, b}$, A. Di Florio ${ }^{a, b}$, F. Errico ${ }^{a, b}$, L. Fiore $^{a}$, A. Gelmi ${ }^{a, b}$, G. Iaselli ${ }^{a, c}$, M. Ince ${ }^{a, b}$, S. Lezki ${ }^{a, b}$, G. Maggi ${ }^{a, c}$, M. Maggi ${ }^{a}$, G. Miniello ${ }^{a, b}, \mathrm{~S}_{\text {. My }}{ }^{a, b}$, S. Nuzzo ${ }^{a, b}$, A. Pompilia, ${ }^{a, b}$ G. Pugliese ${ }^{a, c}$, R. Radogna ${ }^{a}$, A. Ranieri ${ }^{a}$, G. Selvaggi ${ }^{a}, b$, A. Sharma ${ }^{a}$, L. Silvestris ${ }^{a}$, R. Venditti ${ }^{a}$, P. Verwilligen ${ }^{a}$, G. Zito ${ }^{a}$

INFN Sezione di Bologna ${ }^{a}$, Università di Bologna ${ }^{b}$, Bologna, Italy

G. Abbiendi ${ }^{a}$, C. Battilana ${ }^{a, b}$, D. Bonacorsi ${ }^{a}, b$, L. Borgonovi $^{a, b}$, S. Braibant-Giacomelli ${ }^{a, b}$, R. Campanini ${ }^{a, b}$, P. Capiluppi ${ }^{a, b}$, A. Castro ${ }^{a, b}$, F.R. Cavallo ${ }^{a}$, S.S. Chhibra ${ }^{a, b}$, C. Ciocca $^{a}$, G. Codispoti ${ }^{a, b}$, M. Cuffiani ${ }^{a, b}$, G.M. Dallavalle ${ }^{a}$, F. Fabbri ${ }^{a}$, A. Fanfani ${ }^{a, b}$, E. Fontanesi, 
P. Giacomelli ${ }^{a}$, C. Grandi ${ }^{a}$, L. Guiducci ${ }^{a}, b$, F. Iemmi ${ }^{a, b}$, S. Lo Meo ${ }^{a}$, S. Marcellini ${ }^{a}$, G. Masetti $^{a}$, A. Montanari ${ }^{a}$, F.L. Navarria ${ }^{a, b}$, A. Perrotta ${ }^{a}$, F. Primavera ${ }^{a, b, 17}$, T. Rovelli ${ }^{a, b}$, G.P. Siroli ${ }^{a, b}$, N. Tosi ${ }^{a}$

INFN Sezione di Catania ${ }^{a}$, Università di Catania ${ }^{b}$, Catania, Italy

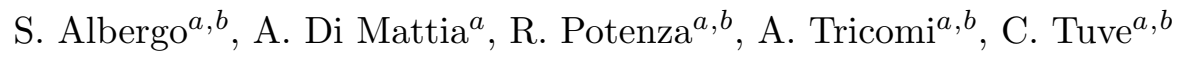

INFN Sezione di Firenze ${ }^{a}$, Università di Firenze ${ }^{b}$, Firenze, Italy

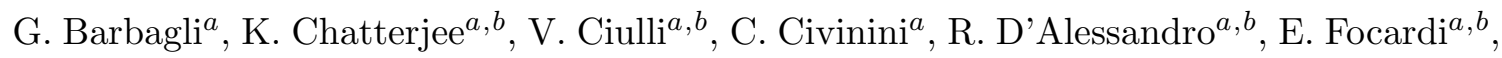

G. Latino, P. Lenzi ${ }^{a}, b$, M. Meschini ${ }^{a}$, S. Paoletti ${ }^{a}$, L. Russo $^{a, 30}$, G. Sguazzoni ${ }^{a}$, D. Strom ${ }^{a}$,

L. Viliani ${ }^{a}$

INFN Laboratori Nazionali di Frascati, Frascati, Italy

L. Benussi, S. Bianco, F. Fabbri, D. Piccolo

INFN Sezione di Genova ${ }^{a}$, Università di Genova ${ }^{b}$, Genova, Italy

F. Ferro ${ }^{a}$, F. Ravera ${ }^{a, b}$, E. Robutti $^{a}$, S. Tosi ${ }^{a, b}$

INFN Sezione di Milano-Bicocca ${ }^{a}$, Università di Milano-Bicocca ${ }^{b}$, Milano, Italy

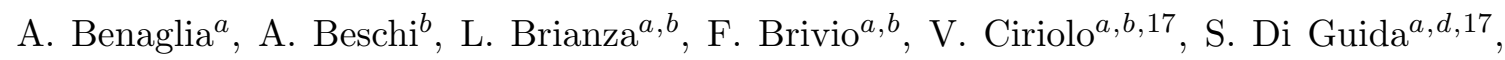
M.E. Dinardo ${ }^{a, b}$, S. Fiorendi ${ }^{a}, b$, S. Gennai ${ }^{a}$, A. Ghezzi ${ }^{a, b}$, P. Govoni $^{a, b}$, M. Malberti $^{a, b}$, S. Malvezzi ${ }^{a}$, A. Massironi ${ }^{a}, b$ D. Menasce ${ }^{a}$, F. Monti, L. Moroni ${ }^{a}$, M. Paganoni $^{a}{ }^{a}$, D. Pedrini ${ }^{a}$, S. Ragazzi ${ }^{a}, b$, T. Tabarelli de Fatis ${ }^{a}, b$ D. Zuolo ${ }^{a, b}$

INFN Sezione di Napoli ${ }^{a}$, Università di Napoli 'Federico II' ${ }^{b}$, Napoli, Italy, Università della Basilicata ${ }^{c}$, Potenza, Italy, Università G. Marconi ${ }^{d}$, Roma, Italy

S. Buontempo ${ }^{a}$, N. Cavallo ${ }^{a, c}$, A. Di Crescenzo ${ }^{a, b}$, F. Fabozzi $^{a, c}$, F. Fienga $^{a}$, G. Galati $^{a}$, A.O.M. Iorio ${ }^{a, b}$, W.A. Khan ${ }^{a}$, L. Lista ${ }^{a}$, S. Meola ${ }^{a, d, 17}$, P. Paolucci ${ }^{a, 17}$, C. Sciacca ${ }^{a, b}$, E. Voevodina $a^{a, b}$

INFN Sezione di Padova ${ }^{a}$, Università di Padova ${ }^{b}$, Padova, Italy, Università di Trento ${ }^{c}$, Trento, Italy

P. Azzi ${ }^{a}$, N. Bacchetta ${ }^{a}$, D. Bisello ${ }^{a, b}$, A. Boletti ${ }^{a, b}$, A. Bragagnolo, R. Carlin ${ }^{a, b}$,

P. Checchia ${ }^{a}$, M. Dall'Osso ${ }^{a}, b$ P. De Castro Manzano ${ }^{a}$, T. Dorigo ${ }^{a}$, U. Dosselli ${ }^{a}$,

F. Gasparini ${ }^{a, b}$, U. Gasparini ${ }^{a, b}$, S.Y. Hoh, S. Lacaprara ${ }^{a}$, P. Lujan, M. Margoni ${ }^{a, b}$, A.T. Meneguzzo ${ }^{a, b}$, J. Pazzini ${ }^{a, b}$, N. Pozzobon ${ }^{a, b}$, P. Ronchese ${ }^{a, b}$, R. Rossin ${ }^{a, b}$, F. Simonetto ${ }^{a, b}$, A. Tiko, E. Torassa ${ }^{a}$, M. Zanetti ${ }^{a, b}$, P. Zotto ${ }^{a, b}$, G. Zumerle ${ }^{a, b}$

INFN Sezione di Pavia ${ }^{a}$, Università di Pavia ${ }^{b}$, Pavia, Italy

A. Braghieri ${ }^{a}$, A. Magnani ${ }^{a}$, P. Montagna ${ }^{a, b}$, S.P. Ratti ${ }^{a}, b, V . \operatorname{Re}^{a}$, M. Ressegotti $^{a, b}$, C. Riccardi ${ }^{a, b}$, P. Salvini ${ }^{a}$, I. Vai ${ }^{a}, b$ P. Vitulo ${ }^{a, b}$

INFN Sezione di Perugia ${ }^{a}$, Università di Perugia ${ }^{b}$, Perugia, Italy

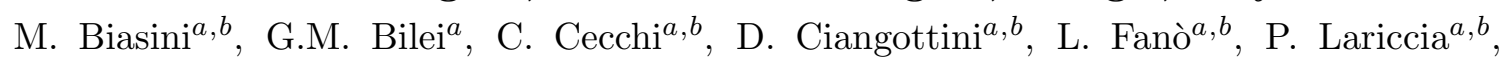

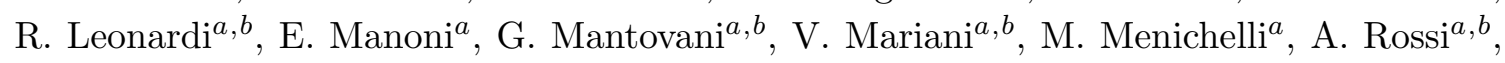
A. Santocchia ${ }^{a, b}$, D. Spiga ${ }^{a}$ 
INFN Sezione di Pisa ${ }^{a}$, Università di Pisa ${ }^{b}$, Scuola Normale Superiore di Pisa ${ }^{c}$, Pisa, Italy

K. Androsov ${ }^{a}$, P. Azzurri ${ }^{a}$, G. Bagliesi ${ }^{a}$, L. Bianchini ${ }^{a}$, T. Boccali ${ }^{a}$, L. Borrello, R. Castaldi ${ }^{a}$, M.A. Ciocci ${ }^{a}, b$, R. Dell'Orso ${ }^{a}$, G. Fedi ${ }^{a}$, F. Fiori ${ }^{a, c}$, L. Giannini $^{a, c}$, A. Giassi $^{a}$, M.T. Grippo ${ }^{a}$, F. Ligabue ${ }^{a, c}$, E. Manca ${ }^{a, c}$, G. Mandorli ${ }^{a, c}$, A. Messineo ${ }^{a, b}$, F. Palla ${ }^{a}$,

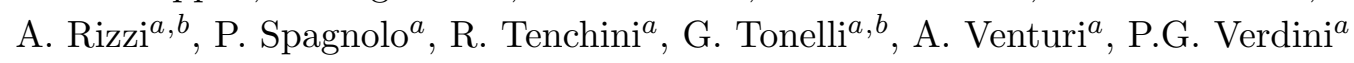

INFN Sezione di Roma ${ }^{a}$, Sapienza Università di Roma ${ }^{b}$, Rome, Italy

L. Barone ${ }^{a, b}$, F. Cavallari ${ }^{a}$, M. Cipriani ${ }^{a, b}$, D. Del Re ${ }^{a, b}$, E. Di Marco ${ }^{a, b}$, M. Diemoz $^{a}$, S. Gelli ${ }^{a, b}$, E. Longo ${ }^{a, b}$, B. Marzocchi ${ }^{a}, b$, P. Meridiani ${ }^{a}$, G. Organtini ${ }^{a, b}$, F. Pandolfi ${ }^{a}$,

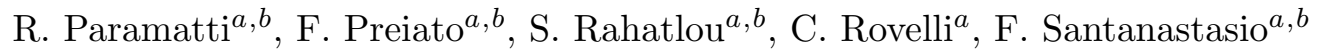

INFN Sezione di Torino ${ }^{a}$, Università di Torino ${ }^{b}$, Torino, Italy, Università del Piemonte Orientale ${ }^{c}$, Novara, Italy

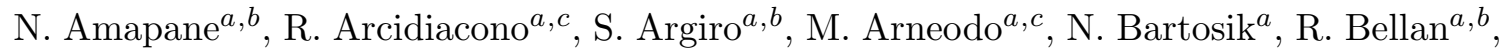
C. Biino ${ }^{a}$, N. Cartiglia ${ }^{a}$, F. Cenna ${ }^{a, b}$, S. Cometti ${ }^{a}$, M. Costa ${ }^{a, b}$, R. Covarelli ${ }^{a, b}$, N. Demaria ${ }^{a}$, B. Kiani ${ }^{a, b}$, C. Mariotti ${ }^{a}$, S. Maselli ${ }^{a}$, E. Migliore ${ }^{a, b}$, V. Monaco ${ }^{a, b}$,

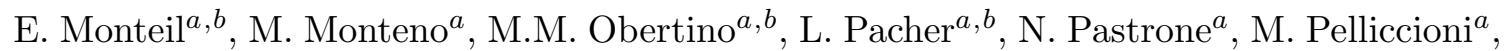
G.L. Pinna Angioni ${ }^{a, b}$, A. Romero ${ }^{a, b}$, M. Ruspa ${ }^{a, c}$, R. Sacchi ${ }^{a, b}$, K. Shchelina ${ }^{a, b}$, V. Sola ${ }^{a}$, A. Solano ${ }^{a, b}$, D. Soldi ${ }^{a, b}$, A. Staiano ${ }^{a}$

INFN Sezione di Trieste ${ }^{a}$, Università di Trieste ${ }^{b}$, Trieste, Italy

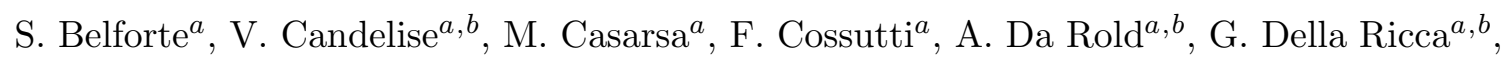
F. Vazzoler ${ }^{a, b}$, A. Zanetti ${ }^{a}$

Kyungpook National University, Daegu, Korea

D.H. Kim, G.N. Kim, M.S. Kim, J. Lee, S. Lee, S.W. Lee, C.S. Moon, Y.D. Oh, S. Sekmen, D.C. Son, Y.C. Yang

Chonnam National University, Institute for Universe and Elementary Particles, Kwangju, Korea

H. Kim, D.H. Moon, G. Oh

Hanyang University, Seoul, Korea

J. Goh ${ }^{31}$, T.J. Kim

Korea University, Seoul, Korea

S. Cho, S. Choi, Y. Go, D. Gyun, S. Ha, B. Hong, Y. Jo, K. Lee, K.S. Lee, S. Lee, J. Lim, S.K. Park, Y. Roh

Sejong University, Seoul, Korea

H.S. Kim

Seoul National University, Seoul, Korea

J. Almond, J. Kim, J.S. Kim, H. Lee, K. Lee, K. Nam, S.B. Oh, B.C. Radburn-Smith, S.h. Seo, U.K. Yang, H.D. Yoo, G.B. Yu

University of Seoul, Seoul, Korea

D. Jeon, H. Kim, J.H. Kim, J.S.H. Lee, I.C. Park 
Sungkyunkwan University, Suwon, Korea

Y. Choi, C. Hwang, J. Lee, I. Yu

Vilnius University, Vilnius, Lithuania

V. Dudenas, A. Juodagalvis, J. Vaitkus

National Centre for Particle Physics, Universiti Malaya, Kuala Lumpur, Malaysia

I. Ahmed, Z.A. Ibrahim, M.A.B. Md Ali ${ }^{32}$, F. Mohamad Idris ${ }^{33}$, W.A.T. Wan Abdullah, M.N. Yusli, Z. Zolkapli

Universidad de Sonora (UNISON), Hermosillo, Mexico

J.F. Benitez, A. Castaneda Hernandez, J.A. Murillo Quijada

Centro de Investigacion y de Estudios Avanzados del IPN, Mexico City, Mexico

H. Castilla-Valdez, E. De La Cruz-Burelo, M.C. Duran-Osuna, I. Heredia-De La Cruz ${ }^{34}$,

R. Lopez-Fernandez, J. Mejia Guisao, R.I. Rabadan-Trejo, M. Ramirez-Garcia,

G. Ramirez-Sanchez, R Reyes-Almanza, A. Sanchez-Hernandez

Universidad Iberoamericana, Mexico City, Mexico

S. Carrillo Moreno, C. Oropeza Barrera, F. Vazquez Valencia

Benemerita Universidad Autonoma de Puebla, Puebla, Mexico

J. Eysermans, I. Pedraza, H.A. Salazar Ibarguen, C. Uribe Estrada

Universidad Autónoma de San Luis Potosí, San Luis Potosí, Mexico

A. Morelos Pineda

University of Auckland, Auckland, New Zealand

D. Krofcheck

University of Canterbury, Christchurch, New Zealand

S. Bheesette, P.H. Butler

National Centre for Physics, Quaid-I-Azam University, Islamabad, Pakistan

A. Ahmad, M. Ahmad, M.I. Asghar, Q. Hassan, H.R. Hoorani, A. Saddique, M.A. Shah, M. Shoaib, M. Waqas

National Centre for Nuclear Research, Swierk, Poland

H. Bialkowska, M. Bluj, B. Boimska, T. Frueboes, M. Górski, M. Kazana, K. Nawrocki, M. Szleper, P. Traczyk, P. Zalewski

Institute of Experimental Physics, Faculty of Physics, University of Warsaw, Warsaw, Poland

K. Bunkowski, A. Byszuk ${ }^{35}$, K. Doroba, A. Kalinowski, M. Konecki, J. Krolikowski, M. Misiura, M. Olszewski, A. Pyskir, M. Walczak 
Laboratório de Instrumentação e Física Experimental de Partículas, Lisboa, Portugal

M. Araujo, P. Bargassa, C. Beirão Da Cruz E Silva, A. Di Francesco, P. Faccioli, B. Galinhas, M. Gallinaro, J. Hollar, N. Leonardo, M.V. Nemallapudi, J. Seixas, G. Strong, O. Toldaiev, D. Vadruccio, J. Varela

\section{Joint Institute for Nuclear Research, Dubna, Russia}

S. Afanasiev, P. Bunin, M. Gavrilenko, I. Golutvin, I. Gorbunov, A. Kamenev, V. Karjavine, A. Lanev, A. Malakhov, V. Matveev ${ }^{36,37}$, P. Moisenz, V. Palichik, V. Perelygin, S. Shmatov, S. Shulha, N. Skatchkov, V. Smirnov, N. Voytishin, A. Zarubin

Petersburg Nuclear Physics Institute, Gatchina (St. Petersburg), Russia

V. Golovtsov, Y. Ivanov, V. Kim ${ }^{38}$, E. Kuznetsova ${ }^{39}$, P. Levchenko, V. Murzin, V. Oreshkin, I. Smirnov, D. Sosnov, V. Sulimov, L. Uvarov, S. Vavilov, A. Vorobyev

Institute for Nuclear Research, Moscow, Russia

Yu. Andreev, A. Dermenev, S. Gninenko, N. Golubev, A. Karneyeu, M. Kirsanov, N. Krasnikov, A. Pashenkov, D. Tlisov, A. Toropin

Institute for Theoretical and Experimental Physics, Moscow, Russia

V. Epshteyn, V. Gavrilov, N. Lychkovskaya, V. Popov, I. Pozdnyakov, G. Safronov, A. Spiridonov, A. Stepennov, V. Stolin, M. Toms, E. Vlasov, A. Zhokin

Moscow Institute of Physics and Technology, Moscow, Russia

T. Aushev

National Research Nuclear University 'Moscow Engineering Physics Institute' (MEPhI), Moscow, Russia

R. Chistov ${ }^{40}$, M. Danilov ${ }^{40}$, P. Parygin, D. Philippov, S. Polikarpov ${ }^{40}$, E. Tarkovskii

P.N. Lebedev Physical Institute, Moscow, Russia

V. Andreev, M. Azarkin ${ }^{37}$, I. Dremin ${ }^{37}$, M. Kirakosyan ${ }^{37}$, S.V. Rusakov, A. Terkulov

Skobeltsyn Institute of Nuclear Physics, Lomonosov Moscow State University, Moscow, Russia

A. Baskakov, A. Belyaev, E. Boos, V. Bunichev, M. Dubinin ${ }^{41}$, L. Dudko, V. Klyukhin,

N. Korneeva, I. Lokhtin, I. Miagkov, S. Obraztsov, M. Perfilov, V. Savrin, A. Snigirev, P. Volkov

Novosibirsk State University (NSU), Novosibirsk, Russia

A. Barnyakov ${ }^{42}$, V. Blinov ${ }^{42}$, T. Dimova ${ }^{42}$, L. Kardapoltsev ${ }^{42}$, Y. Skovpen ${ }^{42}$

Institute for High Energy Physics of National Research Centre 'Kurchatov Institute', Protvino, Russia

I. Azhgirey, I. Bayshev, S. Bitioukov, D. Elumakhov, A. Godizov, V. Kachanov, A. Kalinin,

D. Konstantinov, P. Mandrik, V. Petrov, R. Ryutin, S. Slabospitskii, A. Sobol, S. Troshin, N. Tyurin, A. Uzunian, A. Volkov 
National Research Tomsk Polytechnic University, Tomsk, Russia

A. Babaev, S. Baidali, V. Okhotnikov

University of Belgrade, Faculty of Physics and Vinca Institute of Nuclear Sciences, Belgrade, Serbia

P. Adzic ${ }^{43}$, P. Cirkovic, D. Devetak, M. Dordevic, J. Milosevic

Centro de Investigaciones Energéticas Medioambientales y Tecnológicas (CIEMAT), Madrid, Spain

J. Alcaraz Maestre, A. Álvarez Fernández, I. Bachiller, M. Barrio Luna, J.A. Brochero Cifuentes, M. Cerrada, N. Colino, B. De La Cruz, A. Delgado Peris, C. Fernandez Bedoya, J.P. Fernández Ramos, J. Flix, M.C. Fouz, O. Gonzalez Lopez, S. Goy Lopez, J.M. Hernandez, M.I. Josa, D. Moran, A. Pérez-Calero Yzquierdo, J. Puerta Pelayo, I. Redondo, L. Romero, M.S. Soares, A. Triossi

Universidad Autónoma de Madrid, Madrid, Spain

C. Albajar, J.F. de Trocóniz

Universidad de Oviedo, Oviedo, Spain

J. Cuevas, C. Erice, J. Fernandez Menendez, S. Folgueras, I. Gonzalez Caballero, J.R. González Fernández, E. Palencia Cortezon, V. Rodríguez Bouza, S. Sanchez Cruz, P. Vischia, J.M. Vizan Garcia

Instituto de Física de Cantabria (IFCA), CSIC-Universidad de Cantabria, Santander, Spain

I.J. Cabrillo, A. Calderon, B. Chazin Quero, J. Duarte Campderros, M. Fernandez, P.J. Fernández Manteca, A. García Alonso, J. Garcia-Ferrero, G. Gomez, A. Lopez Virto, J. Marco, C. Martinez Rivero, P. Martinez Ruiz del Arbol, F. Matorras, J. Piedra Gomez, C. Prieels, T. Rodrigo, A. Ruiz-Jimeno, L. Scodellaro, N. Trevisani, I. Vila, R. Vilar Cortabitarte

University of Ruhuna, Department of Physics, Matara, Sri Lanka

N. Wickramage

CERN, European Organization for Nuclear Research, Geneva, Switzerland

D. Abbaneo, B. Akgun, E. Auffray, G. Auzinger, P. Baillon, A.H. Ball, D. Barney, J. Bendavid, M. Bianco, A. Bocci, C. Botta, E. Brondolin, T. Camporesi, M. Cepeda, G. Cerminara, E. Chapon, Y. Chen, G. Cucciati, D. d'Enterria, A. Dabrowski, N. Daci, V. Daponte, A. David, A. De Roeck, N. Deelen, M. Dobson, M. Dünser, N. Dupont, A. Elliott-Peisert, P. Everaerts, F. Fallavollita ${ }^{44}$, D. Fasanella, G. Franzoni, J. Fulcher, W. Funk, D. Gigi, A. Gilbert, K. Gill, F. Glege, M. Guilbaud, D. Gulhan, J. Hegeman, C. Heidegger, V. Innocente, A. Jafari, P. Janot, O. Karacheban ${ }^{20}$, J. Kieseler, A. Kornmayer, M. Krammer ${ }^{1}$, C. Lange, P. Lecoq, C. Lourenço, L. Malgeri, M. Mannelli, F. Meijers, J.A. Merlin, S. Mersi, E. Meschi, P. Milenovic ${ }^{45}$, F. Moortgat, M. Mulders, J. Ngadiuba, S. Nourbakhsh, S. Orfanelli, L. Orsini, F. Pantaleo ${ }^{17}$, L. Pape, E. Perez, M. Peruzzi, A. Petrilli, G. Petrucciani, A. Pfeiffer, M. Pierini, F.M. Pitters, D. Rabady, A. Racz, T. Reis, G. Rolandi ${ }^{46}$, M. Rovere, H. Sakulin, C. Schäfer, C. Schwick, M. Seidel, 
M. Selvaggi, A. Sharma, P. Silva, P. Sphicas ${ }^{47}$, A. Stakia, J. Steggemann, M. Tosi, D. Treille, A. Tsirou, V. Veckalns ${ }^{48}$, M. Verzetti, W.D. Zeuner

Paul Scherrer Institut, Villigen, Switzerland

L. Caminada ${ }^{49}$, K. Deiters, W. Erdmann, R. Horisberger, Q. Ingram, H.C. Kaestli, D. Kotlinski, U. Langenegger, T. Rohe, S.A. Wiederkehr

ETH Zurich - Institute for Particle Physics and Astrophysics (IPA), Zurich, Switzerland

M. Backhaus, L. Bäni, P. Berger, N. Chernyavskaya, G. Dissertori, M. Dittmar, M. Donegà, C. Dorfer, T.A. Gómez Espinosa, C. Grab, D. Hits, J. Hoss, T. Klijnsma, W. Lustermann, R.A. Manzoni, M. Marionneau, M.T. Meinhard, F. Micheli, P. Musella, F. NessiTedaldi, J. Pata, F. Pauss, G. Perrin, L. Perrozzi, S. Pigazzini, M. Quittnat, D. Ruini, D.A. Sanz Becerra, M. Schönenberger, L. Shchutska, V.R. Tavolaro, K. Theofilatos, M.L. Vesterbacka Olsson, R. Wallny, D.H. Zhu

Universität Zürich, Zurich, Switzerland

T.K. Aarrestad, C. Amsler ${ }^{50}$, D. Brzhechko, M.F. Canelli, A. De Cosa, R. Del Burgo, S. Donato, C. Galloni, T. Hreus, B. Kilminster, S. Leontsinis, I. Neutelings, D. Pinna, G. Rauco, P. Robmann, D. Salerno, K. Schweiger, C. Seitz, Y. Takahashi, A. Zucchetta

\section{National Central University, Chung-Li, Taiwan}

Y.H. Chang, K.y. Cheng, T.H. Doan, Sh. Jain, R. Khurana, C.M. Kuo, W. Lin, A. Pozdnyakov, S.S. Yu

\section{National Taiwan University (NTU), Taipei, Taiwan}

P. Chang, Y. Chao, K.F. Chen, P.H. Chen, W.-S. Hou, Arun Kumar, Y.F. Liu, R.-S. Lu, E. Paganis, A. Psallidas, A. Steen

Chulalongkorn University, Faculty of Science, Department of Physics, Bangkok, Thailand

B. Asavapibhop, N. Srimanobhas, N. Suwonjandee

Çukurova University, Physics Department, Science and Art Faculty, Adana, Turkey

A. Bat, F. Boran, S. Cerci ${ }^{51}$, S. Damarseckin, Z.S. Demiroglu, F. Dolek, C. Dozen, I. Dumanoglu, S. Girgis, G. Gokbulut, Y. Guler, E. Gurpinar, I. Hos ${ }^{52}$, C. Isik, E.E. Kangal ${ }^{53}$, O. Kara, A. Kayis Topaksu, U. Kiminsu, M. Oglakci, G. Onengut, K. Ozdemir ${ }^{54}$, S. Ozturk ${ }^{55}$, B. Tali ${ }^{51}$, U.G. Tok, H. Topakli ${ }^{55}$, S. Turkcapar, I.S. Zorbakir, C. Zorbilmez

Middle East Technical University, Physics Department, Ankara, Turkey

B. Isildak ${ }^{56}$, G. Karapinar ${ }^{57}$, M. Yalvac, M. Zeyrek

Bogazici University, Istanbul, Turkey

I.O. Atakisi, E. Gülmez, M. Kaya ${ }^{58}$, O. Kaya ${ }^{59}$, S. Ozkorucuklu ${ }^{60}$, S. Tekten, E.A. Yetkin ${ }^{61}$

Istanbul Technical University, Istanbul, Turkey

M.N. Agaras, A. Cakir, K. Cankocak, Y. Komurcu, S. Sen ${ }^{62}$ 
Institute for Scintillation Materials of National Academy of Science of Ukraine, Kharkov, Ukraine

B. Grynyov

National Scientific Center, Kharkov Institute of Physics and Technology, Kharkov, Ukraine

L. Levchuk

University of Bristol, Bristol, United Kingdom

F. Ball, L. Beck, J.J. Brooke, D. Burns, E. Clement, D. Cussans, O. Davignon, H. Flacher,

J. Goldstein, G.P. Heath, H.F. Heath, L. Kreczko, D.M. Newbold ${ }^{63}$, S. Paramesvaran,

B. Penning, T. Sakuma, D. Smith, V.J. Smith, J. Taylor, A. Titterton

Rutherford Appleton Laboratory, Didcot, United Kingdom

K.W. Bell, A. Belyaev ${ }^{64}$, C. Brew, R.M. Brown, D. Cieri, D.J.A. Cockerill, J.A. Coughlan,

K. Harder, S. Harper, J. Linacre, E. Olaiya, D. Petyt, C.H. Shepherd-Themistocleous,

A. Thea, I.R. Tomalin, T. Williams, W.J. Womersley

\section{Imperial College, London, United Kingdom}

R. Bainbridge, P. Bloch, J. Borg, S. Breeze, O. Buchmuller, A. Bundock, S. Casasso,

D. Colling, P. Dauncey, G. Davies, M. Della Negra, R. Di Maria, Y. Haddad, G. Hall, G. Iles, T. James, M. Komm, C. Laner, L. Lyons, A.-M. Magnan, S. Malik, A. Martelli, J. Nash ${ }^{65}$, A. Nikitenko ${ }^{7}$, V. Palladino, M. Pesaresi, A. Richards, A. Rose, E. Scott, C. Seez, A. Shtipliyski, G. Singh, M. Stoye, T. Strebler, S. Summers, A. Tapper, K. Uchida, T. Virdee ${ }^{17}$, N. Wardle, D. Winterbottom, J. Wright, S.C. Zenz

Brunel University, Uxbridge, United Kingdom

J.E. Cole, P.R. Hobson, A. Khan, P. Kyberd, C.K. Mackay, A. Morton, I.D. Reid, L. Teodorescu, S. Zahid

Baylor University, Waco, U.S.A.

K. Call, J. Dittmann, K. Hatakeyama, H. Liu, C. Madrid, B. Mcmaster, N. Pastika, C. Smith

Catholic University of America, Washington, DC, U.S.A.

R. Bartek, A. Dominguez

The University of Alabama, Tuscaloosa, U.S.A.

A. Buccilli, S.I. Cooper, C. Henderson, P. Rumerio, C. West

Boston University, Boston, U.S.A.

D. Arcaro, T. Bose, D. Gastler, D. Rankin, C. Richardson, J. Rohlf, L. Sulak, D. Zou

Brown University, Providence, U.S.A.

G. Benelli, X. Coubez, D. Cutts, M. Hadley, J. Hakala, U. Heintz, J.M. Hogan ${ }^{66}$, K.H.M. Kwok, E. Laird, G. Landsberg, J. Lee, Z. Mao, M. Narain, S. Sagir ${ }^{67}$, R. Syarif, E. Usai, D. Yu 
University of California, Davis, Davis, U.S.A.

R. Band, C. Brainerd, R. Breedon, D. Burns, M. Calderon De La Barca Sanchez, M. Chertok, J. Conway, R. Conway, P.T. Cox, R. Erbacher, C. Flores, G. Funk, W. Ko, O. Kukral, R. Lander, M. Mulhearn, D. Pellett, J. Pilot, S. Shalhout, M. Shi, D. Stolp, D. Taylor, K. Tos, M. Tripathi, Z. Wang, F. Zhang

University of California, Los Angeles, U.S.A.

M. Bachtis, C. Bravo, R. Cousins, A. Dasgupta, A. Florent, J. Hauser, M. Ignatenko, N. Mccoll, S. Regnard, D. Saltzberg, C. Schnaible, V. Valuev

University of California, Riverside, Riverside, U.S.A.

E. Bouvier, K. Burt, R. Clare, J.W. Gary, S.M.A. Ghiasi Shirazi, G. Hanson, G. Karapostoli, E. Kennedy, F. Lacroix, O.R. Long, M. Olmedo Negrete, M.I. Paneva, W. Si, L. Wang, H. Wei, S. Wimpenny, B.R. Yates

University of California, San Diego, La Jolla, U.S.A.

J.G. Branson, P. Chang, S. Cittolin, M. Derdzinski, R. Gerosa, D. Gilbert, B. Hashemi, A. Holzner, D. Klein, G. Kole, V. Krutelyov, J. Letts, M. Masciovecchio, D. Olivito, S. Padhi, M. Pieri, M. Sani, V. Sharma, S. Simon, M. Tadel, A. Vartak, S. Wasserbaech ${ }^{68}$, J. Wood, F. Würthwein, A. Yagil, G. Zevi Della Porta

University of California, Santa Barbara - Department of Physics, Santa Barbara, U.S.A.

N. Amin, R. Bhandari, J. Bradmiller-Feld, C. Campagnari, M. Citron, A. Dishaw, V. Dutta, M. Franco Sevilla, L. Gouskos, R. Heller, J. Incandela, A. Ovcharova, H. Qu, J. Richman, D. Stuart, I. Suarez, S. Wang, J. Yoo

California Institute of Technology, Pasadena, U.S.A.

D. Anderson, A. Bornheim, J.M. Lawhorn, H.B. Newman, T.Q. Nguyen, M. Spiropulu, J.R. Vlimant, R. Wilkinson, S. Xie, Z. Zhang, R.Y. Zhu

Carnegie Mellon University, Pittsburgh, U.S.A.

M.B. Andrews, T. Ferguson, T. Mudholkar, M. Paulini, M. Sun, I. Vorobiev, M. Weinberg University of Colorado Boulder, Boulder, U.S.A.

J.P. Cumalat, W.T. Ford, F. Jensen, A. Johnson, M. Krohn, E. MacDonald, T. Mulholland, R. Patel, K. Stenson, K.A. Ulmer, S.R. Wagner

Cornell University, Ithaca, U.S.A.

J. Alexander, J. Chaves, Y. Cheng, J. Chu, A. Datta, K. Mcdermott, N. Mirman, J.R. Patterson, D. Quach, A. Rinkevicius, A. Ryd, L. Skinnari, L. Soffi, S.M. Tan, Z. Tao, J. Thom, J. Tucker, P. Wittich, M. Zientek

Fermi National Accelerator Laboratory, Batavia, U.S.A.

S. Abdullin, M. Albrow, M. Alyari, G. Apollinari, A. Apresyan, A. Apyan, S. Banerjee, L.A.T. Bauerdick, A. Beretvas, J. Berryhill, P.C. Bhat, G. Bolla ${ }^{\dagger}$, K. Burkett, J.N. Butler, A. Canepa, G.B. Cerati, H.W.K. Cheung, F. Chlebana, M. Cremonesi, J. Duarte, V.D. Elvira, J. Freeman, Z. Gecse, E. Gottschalk, L. Gray, D. Green, S. Grünendahl, 
O. Gutsche, J. Hanlon, R.M. Harris, S. Hasegawa, J. Hirschauer, Z. Hu, B. Jayatilaka, S. Jindariani, M. Johnson, U. Joshi, B. Klima, M.J. Kortelainen, B. Kreis, S. Lammel, D. Lincoln, R. Lipton, M. Liu, T. Liu, J. Lykken, K. Maeshima, J.M. Marraffino, D. Mason, P. McBride, P. Merkel, S. Mrenna, S. Nahn, V. O’Dell, K. Pedro, C. Pena, O. Prokofyev, G. Rakness, L. Ristori, A. Savoy-Navarro ${ }^{69}$, B. Schneider, E. Sexton-Kennedy, A. Soha, W.J. Spalding, L. Spiegel, S. Stoynev, J. Strait, N. Strobbe, L. Taylor, S. Tkaczyk, N.V. Tran, L. Uplegger, E.W. Vaandering, C. Vernieri, M. Verzocchi, R. Vidal, M. Wang, H.A. Weber, A. Whitbeck

University of Florida, Gainesville, U.S.A.

D. Acosta, P. Avery, P. Bortignon, D. Bourilkov, A. Brinkerhoff, L. Cadamuro, A. Carnes, M. Carver, D. Curry, R.D. Field, S.V. Gleyzer, B.M. Joshi, J. Konigsberg, A. Korytov, K.H. Lo, P. Ma, K. Matchev, H. Mei, G. Mitselmakher, D. Rosenzweig, K. Shi, D. Sperka, J. Wang, S. Wang

Florida International University, Miami, U.S.A.

Y.R. Joshi, S. Linn

Florida State University, Tallahassee, U.S.A.

A. Ackert, T. Adams, A. Askew, S. Hagopian, V. Hagopian, K.F. Johnson, T. Kolberg, G. Martinez, T. Perry, H. Prosper, A. Saha, C. Schiber, R. Yohay

Florida Institute of Technology, Melbourne, U.S.A.

M.M. Baarmand, V. Bhopatkar, S. Colafranceschi, M. Hohlmann, D. Noonan, M. Rahmani, T. Roy, F. Yumiceva

\section{University of Illinois at Chicago (UIC), Chicago, U.S.A.}

M.R. Adams, L. Apanasevich, D. Berry, R.R. Betts, R. Cavanaugh, X. Chen, S. Dittmer, O. Evdokimov, C.E. Gerber, D.A. Hangal, D.J. Hofman, K. Jung, J. Kamin, C. Mills, I.D. Sandoval Gonzalez, M.B. Tonjes, H. Trauger, N. Varelas, H. Wang, X. Wang, Z. Wu, J. Zhang

The University of Iowa, Iowa City, U.S.A.

M. Alhusseini, B. Bilki ${ }^{70}$, W. Clarida, K. Dilsiz ${ }^{71}$, S. Durgut, R.P. Gandrajula, M. Haytmyradov, V. Khristenko, J.-P. Merlo, A. Mestvirishvili, A. Moeller, J. Nachtman, H. Ogul ${ }^{72}$, Y. Onel, F. Ozok ${ }^{73}$, A. Penzo, C. Snyder, E. Tiras, J. Wetzel

Johns Hopkins University, Baltimore, U.S.A.

B. Blumenfeld, A. Cocoros, N. Eminizer, D. Fehling, L. Feng, A.V. Gritsan, W.T. Hung, P. Maksimovic, J. Roskes, U. Sarica, M. Swartz, M. Xiao, C. You

The University of Kansas, Lawrence, U.S.A.

A. Al-bataineh, P. Baringer, A. Bean, S. Boren, J. Bowen, A. Bylinkin, J. Castle, S. Khalil,

A. Kropivnitskaya, D. Majumder, W. Mcbrayer, M. Murray, C. Rogan, S. Sanders, E. Schmitz, J.D. Tapia Takaki, Q. Wang 
Kansas State University, Manhattan, U.S.A.

S. Duric, A. Ivanov, K. Kaadze, D. Kim, Y. Maravin, D.R. Mendis, T. Mitchell, A. Modak, A. Mohammadi, L.K. Saini, N. Skhirtladze

Lawrence Livermore National Laboratory, Livermore, U.S.A.

F. Rebassoo, D. Wright

University of Maryland, College Park, U.S.A.

A. Baden, O. Baron, A. Belloni, S.C. Eno, Y. Feng, C. Ferraioli, N.J. Hadley, S. Jabeen, G.Y. Jeng, R.G. Kellogg, J. Kunkle, A.C. Mignerey, S. Nabili, F. Ricci-Tam, Y.H. Shin, A. Skuja, S.C. Tonwar, K. Wong

\section{Massachusetts Institute of Technology, Cambridge, U.S.A.}

D. Abercrombie, B. Allen, V. Azzolini, A. Baty, G. Bauer, R. Bi, S. Brandt, W. Busza, I.A. Cali, M. D'Alfonso, Z. Demiragli, G. Gomez Ceballos, M. Goncharov, P. Harris, D. Hsu, M. Hu, Y. Iiyama, G.M. Innocenti, M. Klute, D. Kovalskyi, Y.-J. Lee, P.D. Luckey, B. Maier, A.C. Marini, C. Mcginn, C. Mironov, S. Narayanan, X. Niu, C. Paus, C. Roland, G. Roland, G.S.F. Stephans, K. Sumorok, K. Tatar, D. Velicanu, J. Wang, T.W. Wang, B. Wyslouch, S. Zhaozhong

University of Minnesota, Minneapolis, U.S.A.

A.C. Benvenuti, R.M. Chatterjee, A. Evans, P. Hansen, S. Kalafut, Y. Kubota, Z. Lesko, J. Mans, N. Ruckstuhl, R. Rusack, J. Turkewitz, M.A. Wadud

University of Mississippi, Oxford, U.S.A.

J.G. Acosta, S. Oliveros

University of Nebraska-Lincoln, Lincoln, U.S.A.

E. Avdeeva, K. Bloom, D.R. Claes, C. Fangmeier, F. Golf, R. Gonzalez Suarez, R. Kamalieddin, I. Kravchenko, J. Monroy, J.E. Siado, G.R. Snow, B. Stieger

State University of New York at Buffalo, Buffalo, U.S.A.

A. Godshalk, C. Harrington, I. Iashvili, A. Kharchilava, C. Mclean, D. Nguyen, A. Parker, S. Rappoccio, B. Roozbahani

Northeastern University, Boston, U.S.A.

G. Alverson, E. Barberis, C. Freer, A. Hortiangtham, D.M. Morse, T. Orimoto, R. Teixeira De Lima, T. Wamorkar, B. Wang, A. Wisecarver, D. Wood

Northwestern University, Evanston, U.S.A.

S. Bhattacharya, O. Charaf, K.A. Hahn, N. Mucia, N. Odell, M.H. Schmitt, K. Sung, M. Trovato, M. Velasco

University of Notre Dame, Notre Dame, U.S.A.

R. Bucci, N. Dev, M. Hildreth, K. Hurtado Anampa, C. Jessop, D.J. Karmgard, N. Kellams, K. Lannon, W. Li, N. Loukas, N. Marinelli, F. Meng, C. Mueller, Y. Musienko ${ }^{36}$, M. Planer, A. Reinsvold, R. Ruchti, P. Siddireddy, G. Smith, S. Taroni, M. Wayne, A. Wightman, M. Wolf, A. Woodard 
The Ohio State University, Columbus, U.S.A.

J. Alimena, L. Antonelli, B. Bylsma, L.S. Durkin, S. Flowers, B. Francis, A. Hart, C. Hill, W. Ji, T.Y. Ling, W. Luo, B.L. Winer, H.W. Wulsin

Princeton University, Princeton, U.S.A.

S. Cooperstein, P. Elmer, J. Hardenbrook, S. Higginbotham, A. Kalogeropoulos, D. Lange, M.T. Lucchini, J. Luo, D. Marlow, K. Mei, I. Ojalvo, J. Olsen, C. Palmer, P. Piroué, J. Salfeld-Nebgen, D. Stickland, C. Tully

University of Puerto Rico, Mayaguez, U.S.A.

S. Malik, S. Norberg

Purdue University, West Lafayette, U.S.A.

A. Barker, V.E. Barnes, S. Das, L. Gutay, M. Jones, A.W. Jung, A. Khatiwada, B. Mahakud, D.H. Miller, N. Neumeister, C.C. Peng, S. Piperov, H. Qiu, J.F. Schulte, J. Sun, F. Wang, R. Xiao, W. Xie

Purdue University Northwest, Hammond, U.S.A.

T. Cheng, J. Dolen, N. Parashar

Rice University, Houston, U.S.A.

Z. Chen, K.M. Ecklund, S. Freed, F.J.M. Geurts, M. Kilpatrick, W. Li, B.P. Padley, J. Roberts, J. Rorie, W. Shi, Z. Tu, J. Zabel, A. Zhang

University of Rochester, Rochester, U.S.A.

A. Bodek, P. de Barbaro, R. Demina, Y.t. Duh, J.L. Dulemba, C. Fallon, T. Ferbel, M. Galanti, A. Garcia-Bellido, J. Han, O. Hindrichs, A. Khukhunaishvili, P. Tan, R. Taus

Rutgers, The State University of New Jersey, Piscataway, U.S.A.

A. Agapitos, J.P. Chou, Y. Gershtein, E. Halkiadakis, M. Heindl, E. Hughes, S. Kaplan, R. Kunnawalkam Elayavalli, S. Kyriacou, A. Lath, R. Montalvo, K. Nash, M. Osherson, H. Saka, S. Salur, S. Schnetzer, D. Sheffield, S. Somalwar, R. Stone, S. Thomas, P. Thomassen, M. Walker

University of Tennessee, Knoxville, U.S.A.

A.G. Delannoy, J. Heideman, G. Riley, S. Spanier

Texas A\&M University, College Station, U.S.A.

O. Bouhali ${ }^{74}$, A. Celik, M. Dalchenko, M. De Mattia, A. Delgado, S. Dildick, R. Eusebi,

J. Gilmore, T. Huang, T. Kamon ${ }^{75}$, S. Luo, R. Mueller, A. Perloff, L. Perniè, D. Rathjens,

A. Safonov

Texas Tech University, Lubbock, U.S.A.

N. Akchurin, J. Damgov, F. De Guio, P.R. Dudero, S. Kunori, K. Lamichhane, S.W. Lee, T. Mengke, S. Muthumuni, T. Peltola, S. Undleeb, I. Volobouev, Z. Wang

Vanderbilt University, Nashville, U.S.A.

S. Greene, A. Gurrola, R. Janjam, W. Johns, C. Maguire, A. Melo, H. Ni, K. Padeken, J.D. Ruiz Alvarez, P. Sheldon, S. Tuo, J. Velkovska, M. Verweij, Q. Xu 


\section{University of Virginia, Charlottesville, U.S.A.}

M.W. Arenton, P. Barria, B. Cox, R. Hirosky, M. Joyce, A. Ledovskoy, H. Li, C. Neu, T. Sinthuprasith, Y. Wang, E. Wolfe, F. Xia

\section{Wayne State University, Detroit, U.S.A.}

R. Harr, P.E. Karchin, N. Poudyal, J. Sturdy, P. Thapa, S. Zaleski

\section{University of Wisconsin - Madison, Madison, WI, U.S.A.}

M. Brodski, J. Buchanan, C. Caillol, D. Carlsmith, S. Dasu, L. Dodd, B. Gomber, M. Grothe, M. Herndon, A. Hervé, U. Hussain, P. Klabbers, A. Lanaro, K. Long, R. Loveless, T. Ruggles, A. Savin, V. Sharma, N. Smith, W.H. Smith, N. Woods

\section{†: Deceased}

1: Also at Vienna University of Technology, Vienna, Austria

2: Also at IRFU, CEA, Université Paris-Saclay, Gif-sur-Yvette, France

3: Also at Universidade Estadual de Campinas, Campinas, Brazil

4: Also at Federal University of Rio Grande do Sul, Porto Alegre, Brazil

5: Also at Université Libre de Bruxelles, Bruxelles, Belgium

6: Also at University of Chinese Academy of Sciences, Beijing, China

7: Also at Institute for Theoretical and Experimental Physics, Moscow, Russia

8: Also at Joint Institute for Nuclear Research, Dubna, Russia

9: Also at Cairo University, Cairo, Egypt

10: Also at Helwan University, Cairo, Egypt

11: Now at Zewail City of Science and Technology, Zewail, Egypt

12: Also at British University in Egypt, Cairo, Egypt

13: Now at Ain Shams University, Cairo, Egypt

14: Also at Department of Physics, King Abdulaziz University, Jeddah, Saudi Arabia

15: Also at Université de Haute Alsace, Mulhouse, France

16: Also at Skobeltsyn Institute of Nuclear Physics, Lomonosov Moscow State University, Moscow, Russia

17: Also at CERN, European Organization for Nuclear Research, Geneva, Switzerland

18: Also at RWTH Aachen University, III. Physikalisches Institut A, Aachen, Germany

19: Also at University of Hamburg, Hamburg, Germany

20: Also at Brandenburg University of Technology, Cottbus, Germany

21: Also at MTA-ELTE Lendület CMS Particle and Nuclear Physics Group, Eötvös Loránd University, Budapest, Hungary

22: Also at Institute of Nuclear Research ATOMKI, Debrecen, Hungary

23: Also at Institute of Physics, University of Debrecen, Debrecen, Hungary

24: Also at Indian Institute of Technology Bhubaneswar, Bhubaneswar, India

25: Also at Institute of Physics, Bhubaneswar, India

26: Also at Shoolini University, Solan, India

27: Also at University of Visva-Bharati, Santiniketan, India

28: Also at Isfahan University of Technology, Isfahan, Iran

29: Also at Plasma Physics Research Center, Science and Research Branch, Islamic Azad University, Tehran, Iran

30: Also at Università degli Studi di Siena, Siena, Italy

31: Also at Kyunghee University, Seoul, Korea

32: Also at International Islamic University of Malaysia, Kuala Lumpur, Malaysia 
33: Also at Malaysian Nuclear Agency, MOSTI, Kajang, Malaysia

34: Also at Consejo Nacional de Ciencia y Tecnología, Mexico City, Mexico

35: Also at Warsaw University of Technology, Institute of Electronic Systems, Warsaw, Poland

36: Also at Institute for Nuclear Research, Moscow, Russia

37: Now at National Research Nuclear University 'Moscow Engineering Physics Institute' (MEPhI), Moscow, Russia

38: Also at St. Petersburg State Polytechnical University, St. Petersburg, Russia

39: Also at University of Florida, Gainesville, U.S.A.

40: Also at P.N. Lebedev Physical Institute, Moscow, Russia

41: Also at California Institute of Technology, Pasadena, U.S.A.

42: Also at Budker Institute of Nuclear Physics, Novosibirsk, Russia

43: Also at Faculty of Physics, University of Belgrade, Belgrade, Serbia

44: Also at INFN Sezione di Pavia ${ }^{a}$, Università di Pavia ${ }^{b}$, Pavia, Italy

45: Also at University of Belgrade, Faculty of Physics and Vinca Institute of Nuclear Sciences, Belgrade, Serbia

46: Also at Scuola Normale e Sezione dell'INFN, Pisa, Italy

47: Also at National and Kapodistrian University of Athens, Athens, Greece

48: Also at Riga Technical University, Riga, Latvia

49: Also at Universität Zürich, Zurich, Switzerland

50: Also at Stefan Meyer Institute for Subatomic Physics (SMI), Vienna, Austria

51: Also at Adiyaman University, Adiyaman, Turkey

52: Also at Istanbul Aydin University, Istanbul, Turkey

53: Also at Mersin University, Mersin, Turkey

54: Also at Piri Reis University, Istanbul, Turkey

55: Also at Gaziosmanpasa University, Tokat, Turkey

56: Also at Ozyegin University, Istanbul, Turkey

57: Also at Izmir Institute of Technology, Izmir, Turkey

58: Also at Marmara University, Istanbul, Turkey

59: Also at Kafkas University, Kars, Turkey

60: Also at Istanbul University, Faculty of Science, Istanbul, Turkey

61: Also at Istanbul Bilgi University, Istanbul, Turkey

62: Also at Hacettepe University, Ankara, Turkey

63: Also at Rutherford Appleton Laboratory, Didcot, United Kingdom

64: Also at School of Physics and Astronomy, University of Southampton, Southampton, United Kingdom

65: Also at Monash University, Faculty of Science, Clayton, Australia

66: Also at Bethel University, St. Paul, U.S.A.

67: Also at Karamanoğlu Mehmetbey University, Karaman, Turkey

68: Also at Utah Valley University, Orem, U.S.A.

69: Also at Purdue University, West Lafayette, U.S.A.

70: Also at Beykent University, Istanbul, Turkey

71: Also at Bingol University, Bingol, Turkey

72: Also at Sinop University, Sinop, Turkey

73: Also at Mimar Sinan University, Istanbul, Istanbul, Turkey

74: Also at Texas A\&M University at Qatar, Doha, Qatar

75: Also at Kyungpook National University, Daegu, Korea 